\title{
Application of Streamline Simulation for Gas Displacement Processes
}

\author{
Mohamed Nagib CEng, FEI, Chartered Petroleum Engineer \\ Msc Petroleum Engineering \\ Mohamed M. Nagib
}

Submitted in accordance with the requirements for the degree of

Doctor of Philosophy

The University of Leeds

School of Chemical and Process Engineering

December 2019 
The candidate confirms that the work submitted is his own, except where work that has formed part of jointly authored publications has been included. The contribution of the candidate and the other authors to his work has been explicitly indicated below. The candidate confirms that appropriate credit has been given within the thesis where reference has been made to the work of others.

Nagib, M., Burns, A.D., Ingham, D.B., Pourkashanian, M., and El-Banbi A.H., (2014) 'Application of Streamline Simulation for Gas Displacement Processes', Paper SPE 169703 presented at the SPE EOR Conference at Oil and Gas West Asia held in Muscat, Oman, 31 March-2 April 2014.

Nagib, M., Burns, A.D., Ingham, D.B., Pourkashanian, M., and El-Banbi (2014) 'New Application of Streamline Simulation for Gas Displacement Processes', Paper SPE 172261 presented at SPE Annual Caspian Technical Conference and Exhibition, 12-14 November, Astana, Kazakhstan.

Nagib, M., Burns, A.D., Ingham, D.B., Pourkashanian, M., and El-Banbi (2015) 'A New Practice of Streamline Simulation for Compositional and Miscible Floods', Paper SPE 178301 presented at SPE Saudi Arabia Section Annual Technical Symposium and Exhibition, 21-23 April, Al-Khobar, Saudi Arabia

Nagib, M., Burns, A.D., Hassanpour A. and M., El-Banbi (2017). Application of Streamline Simulation to Gas Displacement Processes, International Journal of MultiPhyiscs.

All the work within the publication is directly attributable to the lead author, and the contributions of the other authors was aiding in the interpretation of the results and the actual writing of the paper.

This copy has been supplied on the understanding that it is copyright material and that no quotation from the thesis may be published without proper acknowledgment.

(C)2019

The University of Leeds.

Mohamed Nagib 


\section{Acknowledgments}

Numerous individuals have contributed invaluable assistance and support in making this thesis happen. I would like to start by expressing my gratitude to Dr. Alan Burns for his constant enthusiasm and encouraging words. Also, his sense of humour was particularly helpful at times when it seemed totally impossible to solve a problem. I have learnt a great deal under his guidance and benefited greatly from his supervision. Next, to Professor Ahmed El-Banbi from Cairo University - Petroleum Engineering Department for his continuous valuable guidance and support and the great ideas he suggested for this work. I very much appreciate his incredible insight, dedication and the patience to help me in this research. Next to, Dr Ali Hassanpour deserves special acknowledgement. I very much appreciate his incredible insight, dedication and the patience to help me reach my potential.

I would also like to thank Professor Derek Ingham for his patience and guidance. More thanks go to the fine people in TRG Group, University of Leeds, School of Earth and Environment for their help and friendship.

Finally, my deep thanks to my wife and my kids for their continuous love and their faith in my endeavours. 


\begin{abstract}
Performance evaluation of miscible and near-miscible gas injection processes is available through conventional finite difference (FD) compositional simulation, which is widely used for solving large-scale multiphase displacement problems that always require large computation time. A step can be taken to reduce the time needed by considering low-resolution compositional simulation. The model can be adversely affected by numerical dispersion and may fail to represent geological heterogeneities adequately. The number of fluid components can possibly be reduced at the price of less accurate representation of phase behaviour. Streamline methods have been developed in which fluid is transported along the streamlines instead of the finite difference grid. In streamline-based simulation, a 3D flow problem is decoupled into a set of 1D problems solved along streamlines, reducing simulation time and suppressing any numerical dispersion. Larger time steps and higher spatial resolution can be achieved in these simulations, particularly when sensitivity runs are needed to reduce study uncertainties. Streamline-based reservoir simulation, being orders of magnitude faster than the conventional finite difference methods, may mitigate many of the challenges noted above. For gas injection, the streamline approach could not provide a high resolution or adequate representation for the multiphase displacement.

In this work, the streamline simulations for both compositional and miscible gas injection were tested. In addition, the conventional gas injection scheme and detailed comparison between the FD simulation and the streamline approach are illustrated. A detailed comparison is given between the results of conventional FD simulation and the streamline approach for gas displacement processes. Finally, some guidelines are given on how the streamline method can potentially be used to enhance for gas displacement processes.
\end{abstract}




\section{Table of Contents}

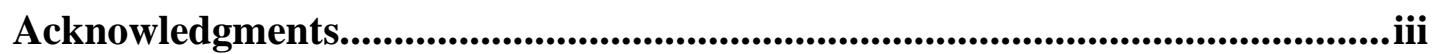

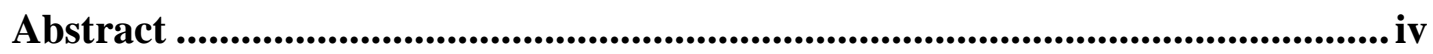

Table of Contents ..................................................................................................

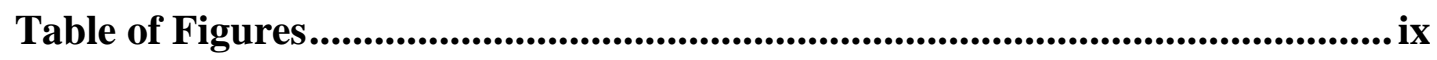

List of Tables ........................................................................................ xviii

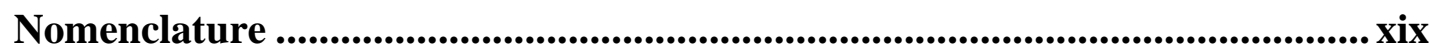

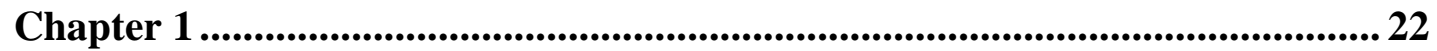

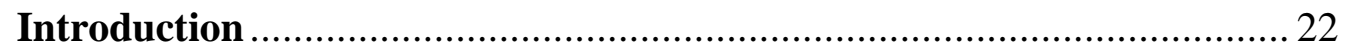

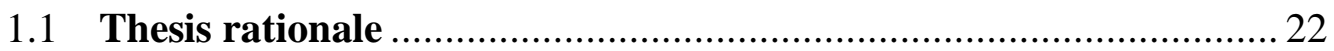

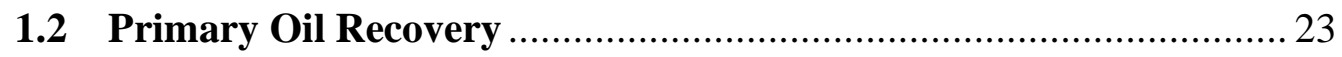

1.2.1 Rock and Liquid Expansion Drive ..................................... 24

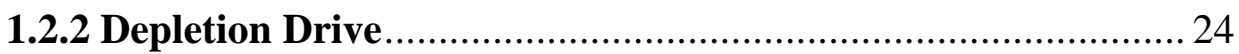

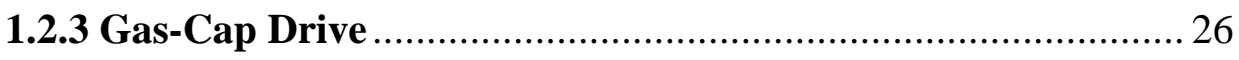

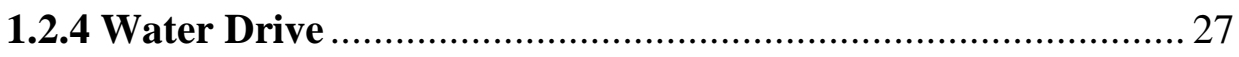

1.2.5 Gravity Drainage Drive .................................................... 31

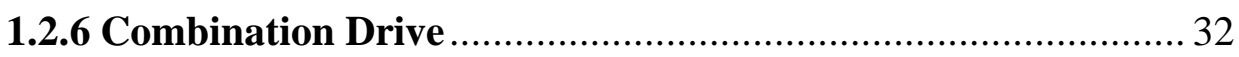

1.2.7 Factors Influencing Combination-Drive Reservoir................. 33

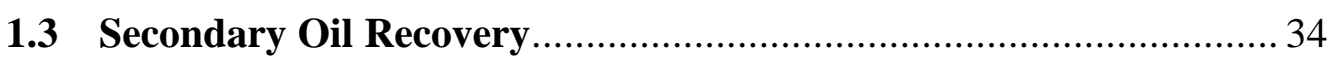

1.3.1 Water Flooding/Water Injection......................................... 37

1.3.2 Gas Displacement Process ...................................................... 38

1.4 Tertiary Recovery /Enhanced Oil Recovery Method .................... 40

1.4.1 Thermally Enhanced Oil Recovery....................................... 40

1.4.2 Miscible Enhanced Oil Recovery ........................................ 41

1.4.3 Chemically Enhanced Oil Recovery .................................... 43

1.5 Reservoir Management and Streamline Simulation ...................... 44

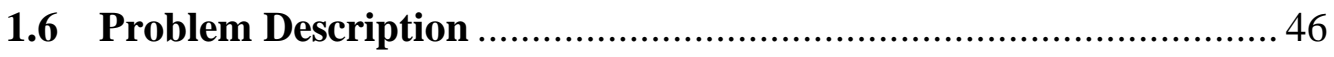

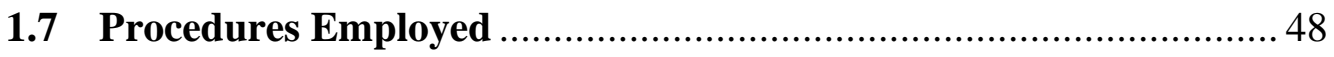

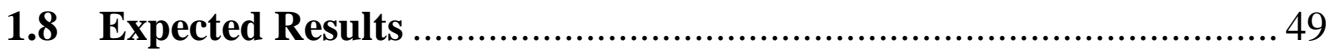

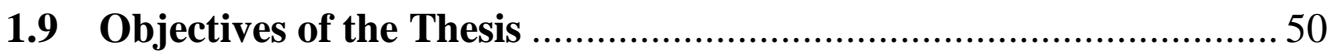

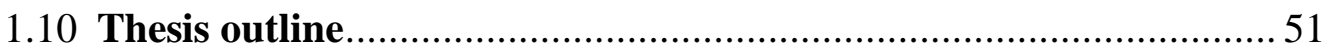

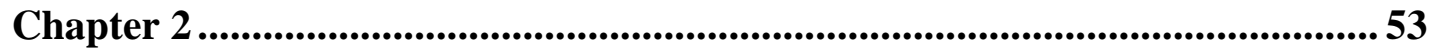

2.1 Introduction to Computational Fluid Dynamics (CFD) ................. 53

2.2 Law of Conservation of Mass .......................................................... 54 
2.3 Single phase 1D Pressure Equation ................................................ 55

2.4 Single Phase Two-Dimensional (2D) Pressure Equation .................. 56

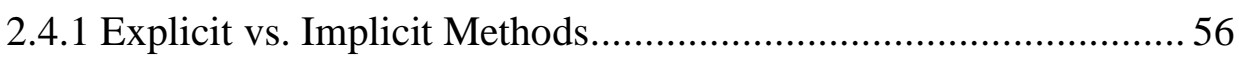

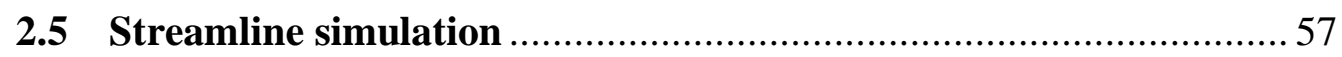

2.5.1 Stream tubes and Streamlines ……................................. 58

2.6 Streamlines in 3D using the Time of Flight Technique................... 62

2.7 Applications of Streamline Simulation........................................... 68

2.7.1 Finite Difference Compositional Simulation.............................. 69

2.7.2 Streamline Compositional Simulation........................................ 69

2.7.3 Compressibility and Streamline Simulation.......................... 71

2.7.4 Innovative Approach by Streamline Simulation ................... 72

2.7.5 Advantages of Streamline Simulation ................................. 73

2.7.6 Disadvantages of Streamline Simulation ............................. 74

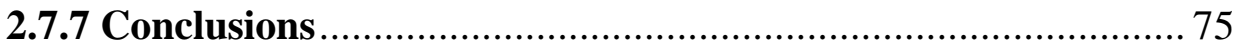

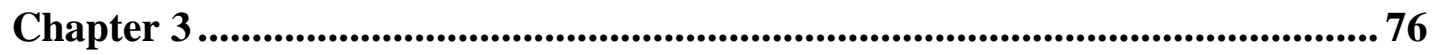

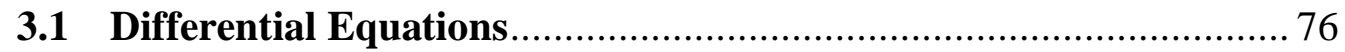

3.1.1 Finite Difference Approximations …...................................... 77

3.1.2 Explicit or Implicit Formulation......................................... 78

3.2 ECLIPSE Reservoir Simulation Software ..................................... 79

3.3 Development of Streamline Simulator (FrontSim) .......................8 80

3.3.1 Streamline Solution Strategy .................................................. 80

3.3.2 The Governing Equations ...................................................... 81

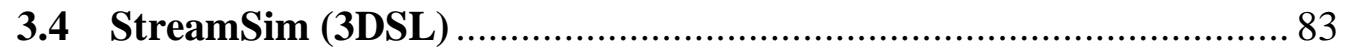

3.5 Frontsim Vs StreamSim (3DSL) .................................................. 84

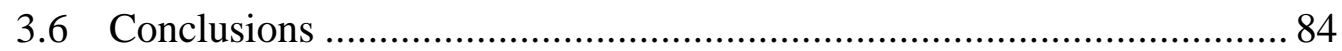

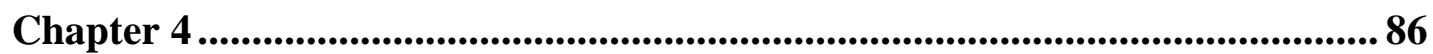

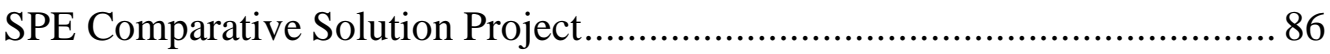

4.1 Description of the Original Tenth SPE Comparative Solution

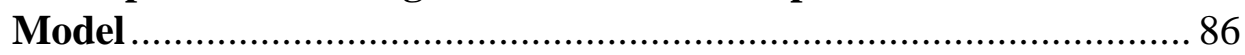

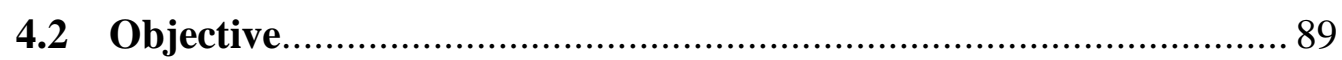

4.3 Enhancement work on the Tenth SPE model ................................ 89

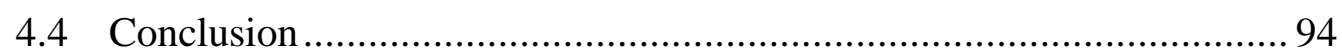




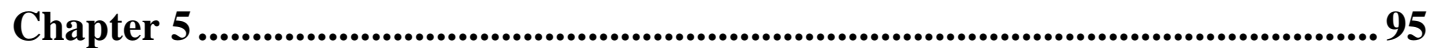

5.1 Research Methodology and Simulation Models............................. 95

5.2 Models Description used for the Research ..................................... 95

5.2.1 Water Flooding Model ........................................................... 97

5.2.2 Water Flooding Model Analysis .......................................... 97

5.2.3 Water flooding Model Performance ........................................ 97

5.2.4 Water Alternating Gas (WAG) Model ................................ 104

5.2.5 WAG Model Performance ..................................................... 106

5.2.6 Gas Injection Model ..................................................... 113

5.2.7 Dead Oil and Live Oil/Dissolved Gas.................................... 113

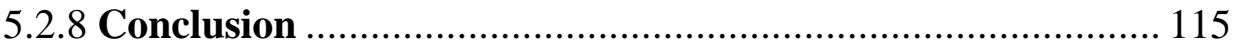

Chapter 6 ................................................................................................................. 116

Baseline Gas Injection Model .......................................................... 116

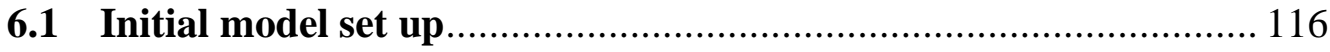

6.2 Streamline sensitivities to improve the flow inside the model ....... 120

6.3 Final Tuning on Time step control ......................................... 125

6.4 Critical Analysis of the Results................................................. 130

6.5 Initial Runs vs. Final Tuned Model ............................................ 131

6.6 Detailed 3D Analysis (Base Case Gas Injection) .......................... 135

6.7 Additional Sensitivities ................................................................. 140

6.7.1 Depletion Case (No Gas injection) ....................................... 140

6.7.2 Gas Injection Rate Sensitivities........................................... 143

6.7.3 Bottom hole Pressure Sensitivities ....................................... 148

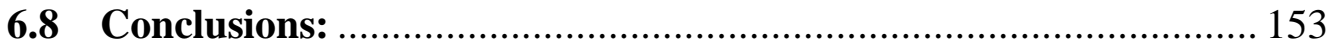

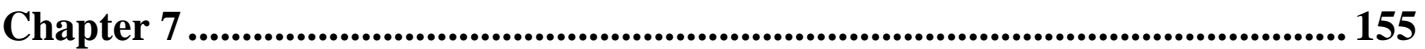

7.1 High Resolution Grid Gas Injection Model .................................... 155

7.2 Analysis of High-Resolution Grid .................................................... 161

7.2.1 3D Analysis (High Resolution Case) ..................................... 167

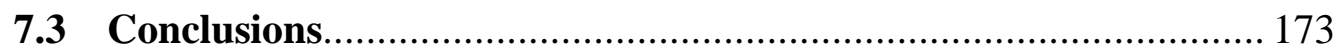

Chapter 8 ...................................................................................................... 174

8.1 Compositional modelling ...................................................... 174

8.1.1 Tunings required for compositional Streamline simulation ........ 176

8.1.2 Analysis of Compositional model.......................................... 180

8.1.3 FD VS SL 3D Analysis (Compositional Model).................... 182 


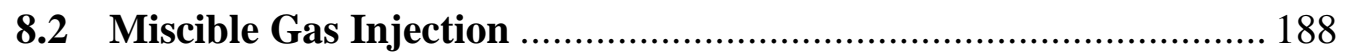

8.3 Analysis of Miscible Flood Model.................................................. 193

8.3.1 FD VS SL 3D Analysis (Miscible Model)............................. 195

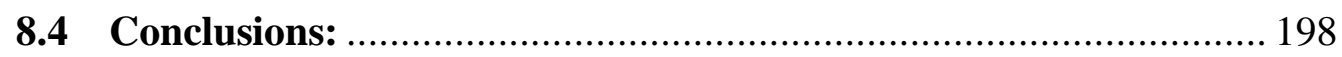

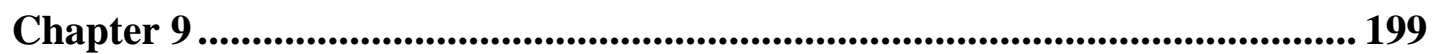

Conclusions and Further Work ........................................................... 199

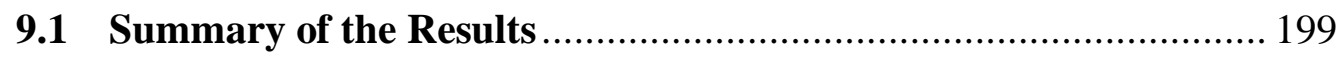

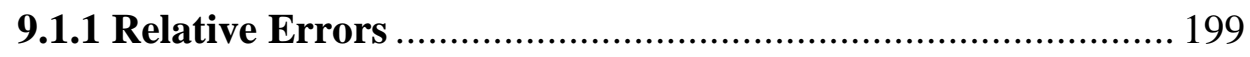

9.1.2 Computational Performance Comparisons:....................... 200

9.1.3 3D Oil Saturation Grid Comparisons................................. 202

9.1.4 3D Gas Saturation Grid Comparisons................................. 205

9.1.5 Oil Thickness Column Analysis (OTC): ............................. 207

9.1.6 Wells Production Data Analysis within 3D Grid:..................... 211

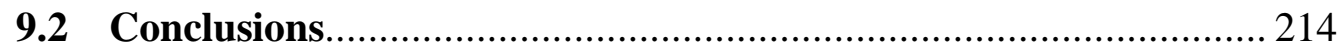

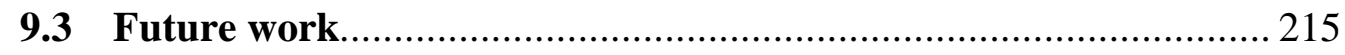

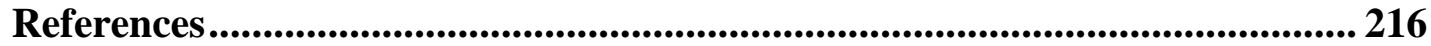




\section{Table of Figures}

Figure 1-1: Schematic of Oil and Gas Recovery methods [5].

Figure 1-2: Schematics of a depleted reservoir (Solution-Gas Drive Reservoir) [12]. 25

Figure 1-3: Schematics of a Gas-cap drive Reservoir [5]. 26

Figure 1-4: Relative positioning of the Gas-Oil contact at the different times [5].

Figure 1-5: Schematic of a water drive mechanism (Artesian water drive) [7]. 28

Figure 1-6: Types of water influx common in the water drive [7].................. 29

Figure 1-7: Reservoir pressure decline in water drive reservoir [5]................. 29

Figure 1-8: Initial fluid distribution in an oil reservoir [5] .......................... 31

Figure 1-9: Combination-drive Reservoir [5] .............................................. 33

Figure 1-10: Schematic of a Typical Water Flood Operation Method [6]. ....... 38

Figure 1-11: A Typical $\mathrm{CO}_{2}$ Gas Injection Method [5] ................................. 39

Figure 1-12: Typical Thermal Recovery Method [7] .................................... 41

Figure 1-13: Typical Miscible EOR Method. [9].......................................... 42

Figure 1-14: Miscible EOR Process showing both Nitrogen and $\mathrm{CO}_{2}$ Injection [9].

Figure 1-15: A Typical chemical EOR method [12] .................................. 43

Figure 1-16: Flow pattern of Injected Chemicals into the Reservoir rocks [9]. 44

Figure 1-17: Flowchart illustrating steps in streamline simulation (Datta-Gupta, 2007)....... 46

Figure 2-1: Fundamentals principles of Flow through Porous Media [12] ....... 55

Figure 2-2: Material Application for the single-Phase Equation in a Control

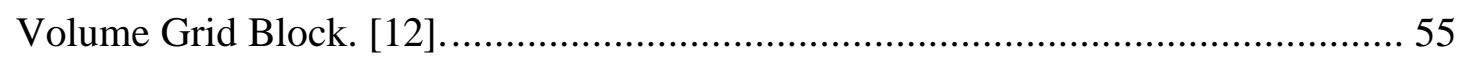

Figure 2-3: The control volume of 2D $x, y$ grid, Block $(i, j)[12] \ldots \ldots \ldots \ldots \ldots \ldots . . . . . . . . .56$

Figure 2-4: Streamline Simulation Flow Diagram [64]................................. 60

Figure 2-5: Comparison of the Full Field History Match Results with the Streamline and Finite Difference Simulators [76] ............................................... 61

Figure 2-6: 2D (right) and 3D (left) that Properly Accounts for the Vertical flow

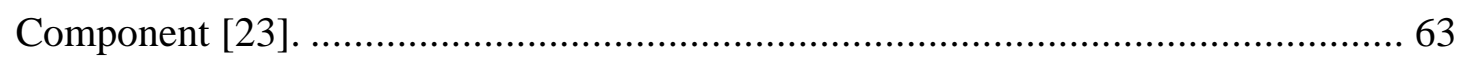

Figure 2-7: The linear Velocity Description in each Coordinate Direction [54]. 64

Figure 2-8: The time it takes for a particle to exit in each coordinate direction

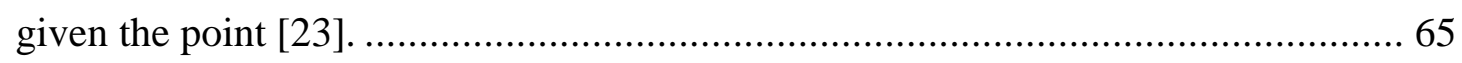

Figure 2-9: The 3D tracing method through a Cartesian Cell [54]................. 65 
Figure 2-10: An arbitrary entry point, the time to exit and the exit point can be

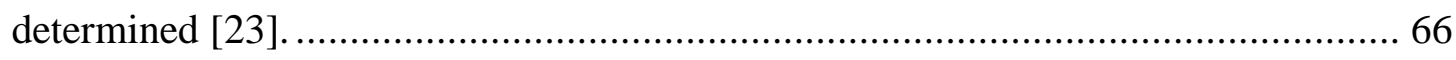

Figure 2-11: the basic algorithm for streamline simulation [75] ................... 67

Figure 2-12: 1D solver and Flux calculation at each time step [73]. ............... 71

Figure 3-1: Block centred grid [15]...................................................... 77

Figure 3-2: Time difference schemes [15]............................................... 79

Figure 4-1: Relative permeability curve generated from Corey's model, with both

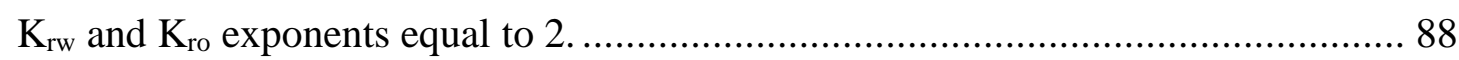

Figure 4-2: Well section showing completion interval for all the wells.......... 90

Figure 4-3: Different vertical permeability upscale for upper Tarbert ............. 91

Figure 4-4: Investigating petrophysical relationships for upscaled properties .. 92

Figure 4-5: Showing Water cut, Oil Production and Bottomhole Pressure for all

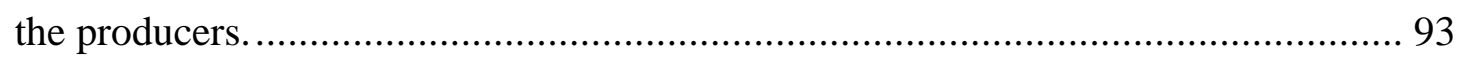

Figure 4-6: Streamlines for water injection for the first-time step................ 93

Figure 5-1: 3D Permeability Distribution and Well Configurations for the Tarbert

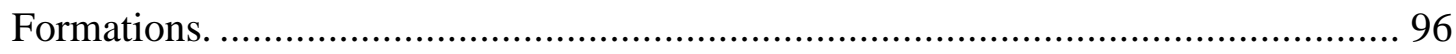

Figure 5-2: Oil Saturation Grid for water flooding at Different Time Steps .... 97

Figure 5-3: Comparison of Oil Production Rates of FD and SL Water Flood Models 98

Figure 5-4: Comparison of total Oil Production of FD and SL Water Flood Models 99

Figure 5-5: Comparison of Water Production Rates of FD and SL Water Flood Models ..... 99

Figure 5-6: Water Cut Performance for Water flooding Model......................100

Figure 5-7: Comparison of Pressure Performance of FD and SL Water Flood Models . 101

Figure 5-8: Total CPU Comparisons for FD and SL Models 102

Figure 5-9: CPU Time Required for Different Solvers used for SL Calculations 102

Figure 5-10: Comparisons of Number of Newton Iterations Calculated for FD and SL Models. 103

Figure 5-11: Comparisons of Number of Linear Iterations Calculated for FD and SL Models 103

Figure 5-12: Total Number of Streamlines Generated for Water Injection Model 
Figure 5-13: Schematic representation of WAG injection Process (U.S Department of Energy, 2013)

Figure 5-14: Oil Saturation Grid for WAG Models at Different Time Steps ...106

Figure 5-15: Comparison of Oil Production Rates of FD and SL WAG Models 107

Figure 5-16: Comparison of total Oil Production of FD and SL WAG Models 107

Figure 5-17: Comparison of Water Production Rates of FD and SL WAG Models 108

Figure 5-18: Water Cut Performance for Water flooding Model. 109

Figure 5-19: Comparison of Pressure Performance of FD and SL WAG Models .109

Figure 5-20: Total CPU Comparisons for FD and SL WAG Models 110

Figure 5-21: CPU Time Required for Different Solvers used for SL Calculations 111

Figure 5-22: Comparisons of Number of Newton Iterations Calculated for FD and SL Models.

Figure 5-23: Comparisons of Number of Linear Iterations Calculated for FD and SL Models. 112

Figure 5-24: Total Number of Streamlines Generated for the WAG SL Model 112

Figure 5-25: Comparison of Oil Production Rates of FD and SL Dead Oil Gas Injection Models. 114

Figure 5-26: Oil and Gas Relative Permeability Data Set used for the Initial Run (Dead Oil). 114

Figure 6-1: 3D Permeability Distribution and Well Configurations for the Tarbert formations. 116

Figure 6-2: Alternative Oil and Gas Relative Permeability data set used (Live Oil) 118

Figure 6-3: Oil Saturation Displayed grid for both FD and SL Gas Injection Models . 118

Figure 6-4: Oil production profile for both FD and SL 119

Figure 6-5: Sensitivity Results of the SL Tuning Parameters with the Relative Error (Oil Rate).

Figure 6-6: Sensitivity results (Oil production profile) for FD and SL simulation.

Figure 6-7: Sensitivity results (Oil production profile) for FD and SL simulation. 
Figure 6-8: Sensitivity results (Oil production profile) for both FD and SL simulation

Figure 6-9: Sensitivity results (Oil production profile) for both Finite Difference and Streamline simulation for the first 200 days.

Figure 6-10: Sensitivity Results (Oil production profile) for both Finite Difference and Streamline simulation for the first 500 days. 128

Figure 6-11: Streamline Simulation Oil saturation Grid at Initial time step before production.

Figure 6-12: Streamline Simulation Oil saturation Grid at the first time step just after start of production.

Figure 6-13: Streamline simulation Oil saturation Grid at the end of Simulation. 130

Figure 6-14: Field Oil Production Rate Differences (old and final) Simulation cases.

Figure 6-15: Field Oil Production Total Relative Error (Initial and final) Simulation cases.

Figure 6-16: Field Gas Production Rate Relative Error (Initial and final) Simulation cases.

Figure 6-17: Field Gas Injection Rates Relative Error (Initial and final) Simulation cases.

Figure 6-18: Field water production rates Relative Error (Initial and final) Simulation cases.

Figure 6-19: Field water cut Relative Error (Initial and final) Simulation cases.

Figure 6-20: Field Pressures Relative Error (Initial and final) Simulation cases. 135

Figure 6-21: First- and Last-time steps 3D Oil saturation Grid (Base Case Model) 136

Figure 6-22: First- and Last-time steps Sliced 3D Oil saturation Grid (Base Case Model) 136

Figure 6-23: First- and Last-time steps of Cross Section 3D Oil saturation Grid (Base Case Model)

Figure 6-24: First- and Last-time steps of sliced 3D Gas saturation Grid (Base Case Model)

Figure 6-25: First- and Last-time steps of a sliced 3D Gas saturation Grid (Base Case Model) .138 
Figure 6-26: First- and Last-time steps of a Cross Section 3D Gas saturation Grid

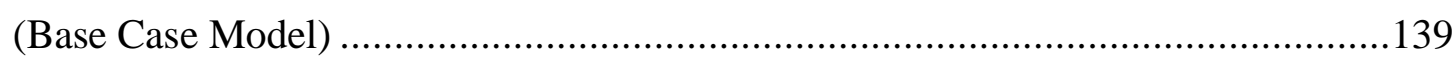

Figure 6-27: OTC of both FD and SL Models...........................................140

Figure 6-28: Oil production rate for both FD and SL (No Gas). ....................141

Figure 6-29: Cumulative Oil production rate for both FD and SL (No Gas)....142

Figure 6-30: Pressure profile for both FD and SL (No Gas).........................143

Figure 6-31: Total CPU required for FD and SL. .....................................143

Figure 6-32: Impact of different gas injection rates on Oil production rate for FD simulation.

Figure 6-33: Impact of different gas injection rates on oil production rate for SL simulation.

Figure 6-34: Impact of different gas injection rates on Cumulative Oil production for FD simulation.

Figure 6-35: Impact of different gas injection rates on Cumulative Oil production for SL simulation. 145

Figure 6-36: Impact of different gas injection rates on Gas Production Rate Performance for FD simulation. 146

Figure 6-37: Impact of different gas injection rates on Gas Production Rate Performance for SL simulation. 146

Figure 6-38: Impact of different gas injection rates on Reservoir Pressure Performance for FD simulation.

Figure 6-39: Impact of different gas injection rates on Reservoir Pressure Performance for SL simulation. 148

Figure 6-40: Impact of different Bottom-hole pressures on oil production rate for FD simulation. 148

Figure 6-41: Impact of different Bottom-hole pressures on oil production rate for SL simulation.

Figure 6-42: Impact of different bottom-hole pressures on cumulative oil production for FD simulation.

Figure 6-43: Impact of Bottom-hole pressures on Cumulative Oil production for SL simulation. 150

Figure 6-44: Impact of Different Bottom-hole Pressures on Gas Production Rate Performance for FD simulation.

Figure 6-45: Impact of Different Bottom-hole Pressures on Gas Production Rate Performance for SL simulation.

Figure 6-46: Impact of Different Bottom-hole Pressures on Gas Injections Rates for FD simulation. 
Figure 6-47: Impact of Different Bottom hole Pressures on Gas Injection Rates for SL simulation. 152

Figure 6-48: Impact of Different Bottom-hole Pressures on Reservoir Pressure Performance for FD simulation. 152

Figure 6-49: Impact of different bottom-hole pressures on Reservoir Pressure Performance for SL simulation. 153

Figure 7-1: High Resolution Porosity Grid vs. Low Resolution. 155

Figure 7-2: Permeability cross section of Fine Vs Cparse Grid. 156

Figure 7-3: Permeability Histogram of the coarse Vs fine Grid. .156

Figure 7-4: Oil production rate for both FD and SL (High Resolution Grid)...157

Figure 7-5: Cumulative Oil production rate for both FD and SL (High Resolution Grid). . 158

Figure 7-6: Pressure profiles for both FD and SL (High Resolution Grid).....159

Figure 7-7: Gas Injection Rate Profiles for both FD and SL (High Resolution Grid). 159

Figure 7-8: Gas Production Rate Profile for both FD and SL (High Resolution Grid).

Figure 7-9: Water Production Profiles for both FD and SL (High Resolution Grid). 161

Figure 7-10: Total CPU required for FD and SL (High Resolution Grid) 161

Figure 7-11: Relative Error in Oil Production Rate between FD and SL Simulation cases (High Resolution Grid).

Figure 7-12: Relative Error of Total Oil Production between FD and SL Simulation cases (High Resolution Grid).

Figure 7-13: Relative Error of Gas Production Rate between FD and SL Simulation cases (High Resolution Grid).

Figure 7-14: Relative Error of Gas Production Total between FD and SL Simulation cases (High Resolution Grid). 164

Figure 7-15: Relative Error of Total Gas Injection between FD and SL simulation models (High Resolution).

Figure 7-16: Relative Error of bottom hole pressure between FD and SL simulation models (High Resolution). .165

Figure 7-17: Oil Production Rates before and after improvement for both FD and SL models of the High-Resolution Grid. 166

Figure 7-18: First- and Last-time steps 3D Oil saturation Grid (High Resolution FD Model) .167 
Figure 7-19: First- and Last-time steps 3D Oil saturation Grid (High Resolution SL Model) 168

Figure 7-20: First- and Last-time steps of cross section 3D Oil saturation Grid (High Resolution Model) 169

Figure 7-21: First- and Last-time steps of 3D Gas Saturation Grid (High Resolution Model).

Figure 7-22: First- and Last-time steps of sliced 3D Gas Saturation Grid (High Resolution Model).

Figure 7-23: First- and Last-time steps of Cross section 3D Gas Saturation Grid (High Resolution Model) .172

Figure 7-24: High Resolution Grid OTC of both FD and SL Models. 173

Figure 8-1: Compositional streamline simulator flow chart. 175

Figure 8-2: Compositional modelling relative permeability data used in both FD and SL simulations. 176

Figure 8-3: Oil production rate for both FD and SL (Compositional).

Figure 8-4: Cumulative Oil production for both FD and SL (Compositional) 177

Figure 8-5: Gas Production rate for both FD and SL (Compositional) ............178

Figure 8-6: Gas Injection Rate for both FD and SL (Compositional).............179

Figure 8-7: Total CPU required for FD and SL (Compositional). 179

Figure 8-8: Relative Error Differences in Oil Production Rate between FD and SL Simulation cases (Compositional). 180

Figure 8-9: Relative Error in total Oil Production between FD and SL Simulation cases (Compositional). 181

Figure 8-10: Relative Error in Gas Production between FD and SL Simulation cases (Compositional). 181

Figure 8-11: Relative Error on Gas Injection Rate between FD and SL simulation models (Compositional). 182

Figure 8-12: 3D Oil saturation Grid for Compositional Modelling at 1st and Last Time step for Both FD and SL. 183

Figure 8-13: Sliced Oil saturation Grid for Compositional Modelling at 1st and Last Timestep for Both FD and SL. 183

Figure 8-14: Oil saturation Grid cross section for Compositional Model at 1st and Last Time Step.

Figure 8-15: 3D Gas Saturation Grid for Compositional Modelling at $1^{\text {st }}$ and Last Timestep for Both FD and SL.... 185

Figure 8-16: Sliced Gas saturation Grid for Compositional Modelling at $1^{\text {st }}$ and Last Timestep for Both FD and SL. 186 
Figure 8-17: Gas Saturation Grid cross section for Compositional Model at $1^{\text {st }}$ and Last Timestep.

Figure 8-18: Compositional model OTC at the end of field life of both FD and SL models.....

Figure 8-19: Miscible gas injection relative permeability data used in both FD and SL simulations.

Figure 8-20: Oil production Rates for both FD and SL (Miscible Gas)

Figure 8-21: Cumulative Oil production for both FD and SL (Miscible Gas) 190

Figure 8-22: Gas Production rates for both FD and SL (Miscible Gas) ...........191

Figure 8-23: Gas Injection Rate for both FD and SL (Miscible Gas).

Figure 8-24: Reservoir Pressure Performance for both FD and SL (Miscible Gas).

Figure 8-25: Total CPU required for FD and SL (Miscible Gas) 192

Figure 8-26: Relative Error in Oil Production Rate between FD and SL Simulation cases (Miscible Gas).

Figure 8-27: Relative Error in Cumulative Oil Production between FD and SL Simulation cases (Miscible Gas).

Figure 8-28: Relative Error in Gas Production between FD and SL Simulation cases (Miscible Gas).

Figure 8-29: Relative Error in Gas Injection Rates between FD and SL Simulation cases (Miscible Gas).

Figure 8-30: 3D Oil saturation Grid for Miscible Flood Model at $1^{\text {st }}$ and Last Timestep 195

Figure 8-31: Sliced Oil saturation Grid for Miscible Flood Model at $1^{\text {st }}$ and Last Timestep

Figure 8-32: Oil saturation Grid cross section for Miscible Flood Model at $1^{\text {st }}$ and Last Timestep 196

Figure 8-33: Gas Saturation Grid for Miscible Flood Model at 1st and Last Timestep

Figure 8-34: Sliced Gas Saturation Grid for Miscible Flood Model at $1^{\text {st }}$ and Last Timestep 197

Figure 8-35: First- and Last-time steps of sliced 3D Gas saturation Grid (Miscible Flood FD Model). 197

Figure 8-36: Miscible Flood Model OTC at the end of field life of both FD and SL models 198

Figure 9-1: Relative Error \% between FD and SL Models 200 
Figure 9-2: Total CPU vs. Time for Gas injection models in FD and SL. ......201

Figure 9-3: Comparison of Total CPU for Gas injection models in FD and SL.

Figure 9-4: 3D Oil Saturation Grid Comparisons of Black Oil Base for both FD and SL cases. 203

Figure 9-5: 3D Oil Saturation Grid Comparisons of High-Resolution case for both FD and SL cases.

Figure 9-6: 3D Oil Saturation Grid Comparisons of the Compositional case for both FD and SL cases. 204

Figure 9-7: 3D Oil Saturation Grid Comparisons of Miscible flood model for both FD and SL cases. .205

Figure 9-8: 3D Gas Saturation Grid Comparisons of Black Oil Base for both FD and SL cases. 205

Figure 9-9: 3D Gas Saturation Grid Comparisons of High-Resolution model for both FD and SL cases. .206

Figure 9-10: 3D Gas Saturation Grid Comparisons of Compositional model for both FD and SL cases. .207

Figure 9-11: 3D Gas Saturation Grid Comparisons of Miscible Flood model for both FD and SL cases. 207

Figure 9-12: OTC Differences of the base Case Gas Injection Model............208

Figure 9-13: OTC Differences of High-Resolution Gas Injection Model.......209

Figure 9-14: OTC Differences of Compositional Gas Injection Model...........210

Figure 9-15: OTC Differences of Miscible Flood Gas Injection Model. .........211

Figure 9-16: Total oil production per each well with 3D grid of the base case model.

Figure 9-17: Total oil production per each well with 3D grid of the high-resolution model. . 212

Figure 9-18: Total oil production per each well with 3D grid of the compositional model. 213

Figure 9-19: Total oil production per each well with 3D grid of the Miscible model. 


\section{List of Tables}

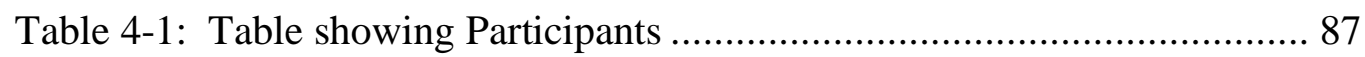

Table 4-2: PVT property of under saturated oil used in model 2................... 88

Table 5-1 : Oil and Gas Relative Permeability Data (Dead Oil).....................115

Table 6-1: Alternative Oil and Gas Relative Permeability Data Set used for the

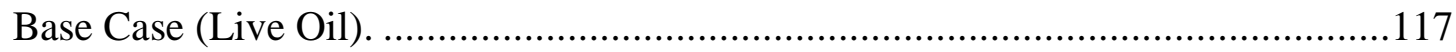

Table 6-2: Streamline simulation control output tuning parameters:..............123

Table 8-1: Compositional PVT data set......................................................175

Table 9-1: Summaries of the relative error between FD and SL in Simulation

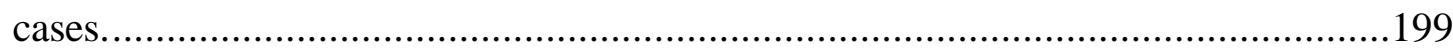

Table 9-2: Summaries Total CPU used by FD and SL...............................201 


\section{Nomenclature}

$\begin{array}{ll}\text { Abbreviation } & \\ \text { CFD } & \text { Computation Fluid Dynamics } \\ \text { CFL } & \text { Courant-Fredrich-Levy } \\ \text { EOR } & \text { Enhanced Oil Recovery } \\ \text { EOS } & \text { Equation of State } \\ \text { FD } & \text { Finite Difference } \\ \text { LPG } & \text { Liquefied Petroleum Gases } \\ \text { OTC } & \text { Oil Thickness Column } \\ \text { SAGD } & \text { Steam Assisted Gravity Drainage } \\ \text { SL } & \text { Streamline } \\ \text { Sor } & \text { Residual Oil Saturation } \\ \text { TOF } & \text { Time of Flight } \\ \text { WAF } & \text { Well rate allocation factors } \\ \text { WAG } & \text { Water Alternate Gas }\end{array}$

\section{Roman Symbols}

A

$b$

C

$f$

$g$

$\mathrm{k}$

$\mathrm{K}_{\mathrm{r}}$

M

$\mathrm{P}$

$\mathrm{P}_{\mathrm{c}}$
Area

Formation Volume Factor

Compressibility

Conserved Fluxes

Acceleration due to gravity

Permeability

Relative Permeability

Mobility

Pressure

Capillary Pressure 


$\begin{array}{ll}Q & \text { Flow Rate } \\ R & \text { Dissolved Gas } \\ S & \text { Saturation } \\ t & \text { Time } \\ V & \text { Velocity } \\ \mathrm{V} & \text { Interstitial Velocity } \\ C & \text { Compressibility } \\ x & \text { X direction } \\ y & \text { Y Direction } \\ z & \text { Z Direction }\end{array}$

\section{Greek Symbols}

$\Delta t$

$\Delta x$

$\Delta \mathrm{y}$

$\Delta \mathrm{z}$

$\varphi$

$\mu$

$\rho$

\section{Subscripts}

$g$

$o$

w
Change in $t$

Change in $x$

Change in $y$

Change in $\mathrm{z}$

Volume Fraction

Molecular Viscosity

Density

Gas

Oil

Water 


\section{Chemical symbols}

$\mathrm{C}$

$\mathrm{C} 12$

$\mathrm{CO}$

$\mathrm{CO}_{2}$

$\mathrm{H}_{2} \mathrm{O}$

$\mathrm{O}_{2}$
Carbon

Carbon-12

Carbon monoxide

Carbon dioxide

Water molecule

Oxygen molecule 


\section{Chapter 1}

\section{Introduction}

\subsection{Thesis rationale}

The production of oil from most commercially viable reservoirs is generally described by three stages termed natural recovery, supplementary recovery and enhanced recovery processes. The recovery processes are used in consecutive order and the shift between methods is usually governed by whether the subsequent production method is economic or not. The production of oil has a very wide range of efficiency, described as the recovery factor (RF). No matter whether any single recovery process is efficient or not, the three terms are used by convention from the initial planning stage. For instance, it is also worth noting that the use of enhanced methods without the use of supplementary methods may well still improve the overall recovery factor in the field after the primary recovery method has been either physically or equally economically exhausted.

Natural recovery, which is the same as the primary production, uses the natural drive energy locked up in the reservoir and surroundings to displace the oil from the porous rocks towards the production wells. Natural reservoir energy forces oil to the producing wells but with a decrease in the reservoir pressure. Due to this decrease in the reservoir pressure, the new in situ reservoir pressure may drop below the bubble point pressure, whereby dissolved gases are released from the oil [12][2].

Natural recovery extracts only about $25-40 \%$ of the original oil in place (OOIP). Generally, this means that natural drive mechanisms leave behind around $60-75 \%$ of the oil initially in place.

Secondary methods will generally increase the natural energy by the fluid injection - see Figure1.1. In so-called displacement recovery methods, volumes of injected fluid interact with the reservoir fluid to move reservoir fluid from one part of the reservoir to another. In order to maintain the reservoir pressure and also to sweep out oil from the porous rocks in a more efficient way, a common displacement process known as water flooding is used in many reservoir formations since this is a low cost and simple secondary method. For petroleum engineers, if sufficient information is available, engineers will be able to simulate and approximate reservoir conditions through modelling in order to know what best fits the reservoir in terms of efficient drainage. However, it is analytically impossible to exactly simulate any given natural reservoir due to the number of parameters that occur in the model, along with the associated uncertainty of various parameters.

Displacement methods are not always efficient, when high volumes of injected fluid may only displace a relatively small fraction of hydrocarbons. In particular, the inefficient displacement of the water 
flooding mechanism is often due to the unfavourable mobility ratio or reservoir heterogeneity. Research has established that the mobility of brine used in water flooding is highly reduced by adding small amounts of hydrolysed polyacrylamide, a water-soluble polymer. The reduction in the brine mobility results in greater oil recovery when compared to the conventional water flooding technique [3][4][5].

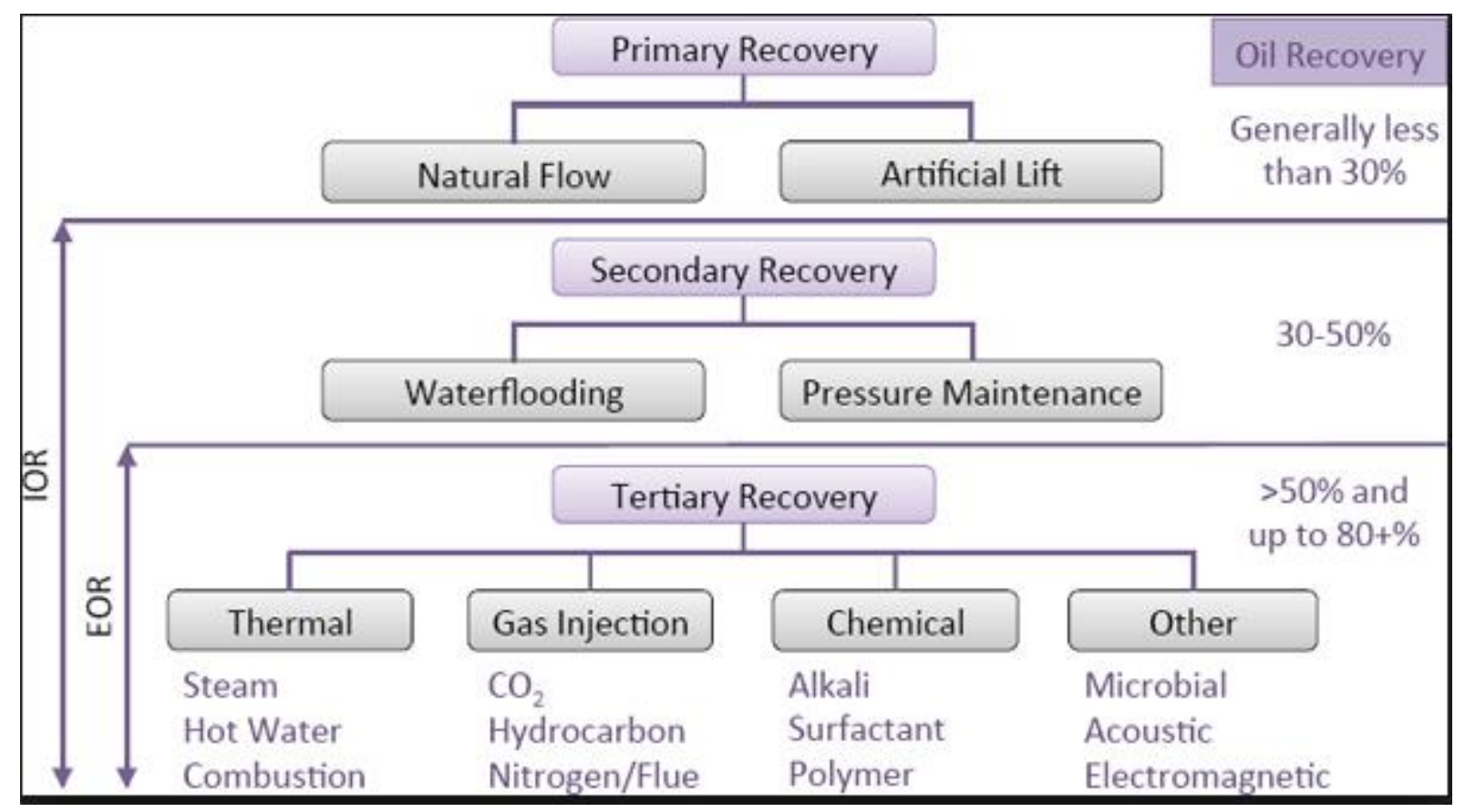

Figure 1-1: Schematic of Oil and Gas Recovery methods [5].

Hence, production plans increasingly include enhanced oil recovery (EOR) processes to mobilise oil in the reservoir rock.

\subsection{Primary Oil Recovery}

Primary oil recovery, also known as the natural reservoir drive mechanism, consists of different types of reservoir drive mechanisms, which displace the oil towards the producing well or well bore. Generally, there are six types of reservoir drive mechanism:

- Rock and liquid expansion drive

- Depletion drive

- Gas cap drive

- Water drive

- Gravity drainage drive

- Combination drive 


\subsubsection{Rock and Liquid Expansion Drive}

When an oil reservoir exists initially at a pressure higher than the bubble point pressure, the reservoir is known as an 'under-saturated oil reservoir'. At pressures above the bubble-point pressure, crude oil, connate water and rock are the only materials present. Whenever the reservoir pressure goes on to decline with increasing production from the reservoir, both rock material and fluids can then expand due to their individual compressibility [5][6].

The rock compressibility responds because of two factors:

- Expansion of individual rock grains

- Formation compaction.

The factors arise as a result of the decrease in fluid pressure within the pore spaces, and both tend to reduce the pore volume through the resultant reduced porosity. Further expansion of the fluids and reduction in the pore volume occurs with decreases in reservoir pressure. The crude oil and water will then be forced out of the pore space and into the well bore. Since liquids and rocks are only slightly compressible, the reservoir will experience a sharp pressure decline [5][6].

The reservoir under such a drive mechanism is characterised by a constant gas-oil ratio that is equal to the gas solubility at the bubble point pressure. This drive mechanism is considered the least efficient of all the drive mechanisms, when the result of the recovery of oil from the total oil in place is generally reduced in comparison with other mechanisms [5][6].

\subsubsection{Depletion Drive}

This drive mechanism can also be referred to by the following terms:

- Solution gas drive

- Dissolved gas drive

- Internal gas drive.

In this type of reservoir, the main source of energy is a result of gas liberation from the crude oil and further expansion of solution gas as the reservoir pressure decreases. As the pressure falls below bubblepoint pressure, gas bubbles are liberated within the microscopic pore spaces. The liberated bubbles of gas expand and force petroleum liquids as well as gas out of the pore space [5][6]. 
Depletion drives generally exhibit the following characteristics:

Reservoir Pressure: Reservoir pressure declines rapidly and continuously. The reservoir pressure behaviour is linked to the fact that no extraneous fluids or gas caps are available to provide any replacement of gas and oil withdrawals.

Water Production: During the life of the reservoir, water production is much less visible; meaning the production of oil comes without associated water during the production lifecycle.

Gas-Oil ratio: A rapid increase in gas-oil ratio on the producing wells is usually a characteristic of a depletion drive reservoir. As soon as the reservoir pressure decreases below bubble point pressure, gas evolves from solution throughout the reservoir. Once the gas saturation then exceeds the critical gas saturation, free gas begins to flow toward the wellbore and the gasoil ratio increases.

Ultimate Oil Recovery: Oil production in a depletion drive often has a weak recovery efficiency of about 5-30\%, with large quantities of oil otherwise being left in the reservoir. Therefore, depletion drive reservoirs are considered the most suitable sources for secondary recovery application [7].

Figure 1-2 shows a schematic diagram of a depleted reservoir (Solution Gas Drive).

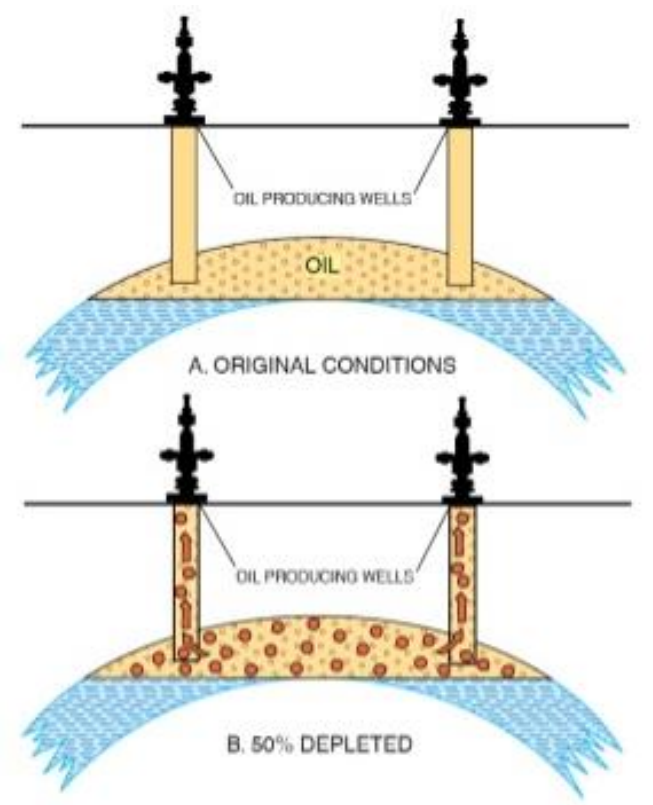

Figure 1-2: Schematics of a depleted reservoir (Solution-Gas Drive Reservoir) [12]. 


\subsubsection{Gas-Cap Drive}

A gas-cap drive reservoir is characterised by the presence of the gas-cap with little or no water drive as shown in Figure 1-3. With the ability of the gas-cap to expand, the reservoir is characterised by a slow decline in reservoir pressure [5][7].

The natural energy available to produce the crude oil comes from two sources:

- Expansion of the gas-cap gas

- Expansion of the solution gas as it is liberated

A schematic of a gas-cap reservoir is shown in Figure 1-3.

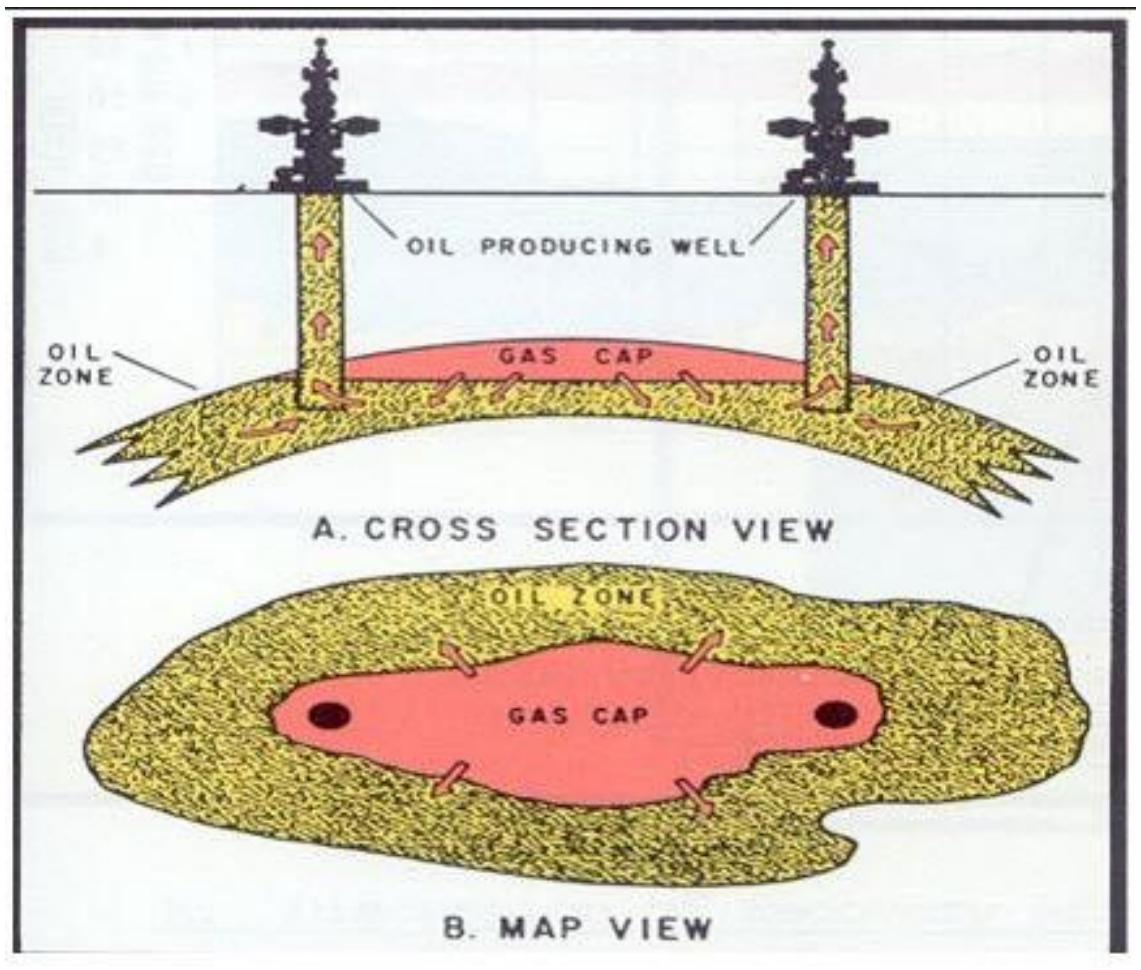

Figure 1-3: Schematics of a Gas-cap drive Reservoir [5].

The characteristics of a gas-cap drive reservoir are as follows:

Reservoir pressure: The reservoir pressure is usually dropped continuously and slowly. Pressure is required to be maintained at a higher level than in a depletion drive reservoir. The 
degree of pressure maintenance depends upon the volume of gas in the gas cap compared to the oil volume.

Water production: Absent or negligible water production.

Gas-oil ratio: The gas-oil ratio rises continuously in up-structure wells. Gas-oil ratio usually increases as the expanding gas cap reaches the producing intervals of up structure wells.

Ultimate oil recovery: Oil recovery by gas-cap expansion is actually a frontal drive displacing mechanism that, therefore, leads to a considerably larger recovery efficiency factor than that of depletion-drive reservoirs. This larger recovery efficiency is mainly due to the fact that no gas saturation is being formed throughout the reservoir at the same time.

Figure 1-4 shows the relative positions of the gas-oil contact at different times in the producing life of the reservoir. The expected oil recovery ranges from $20-40 \%$.

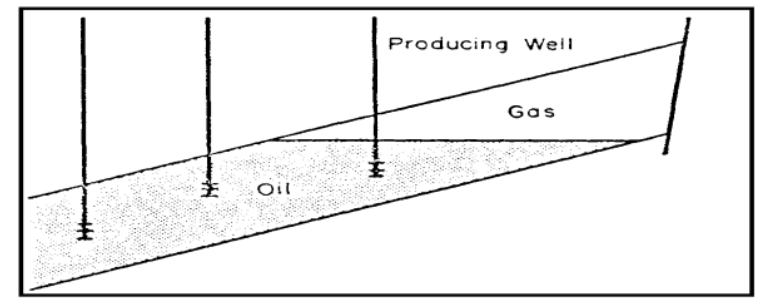

A. Initial fluid distribution.

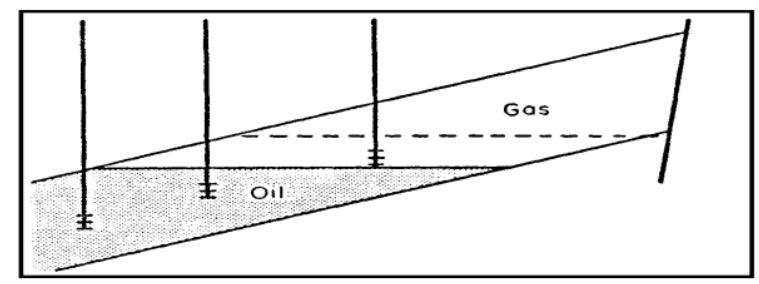

Figure 1-4: Relative positioning of the Gas-Oil contact at the different times [5].

Well behaviour: Due to the effects of gas-cap expansion on maintaining reservoir pressure and the effect of the decreased liquid column height and hence weight, as oil is produced from the well, gas-cap-drive reservoirs tend to flow for longer period than depletion-drive reservoirs.

\subsubsection{Water Drive}

Most reservoirs are bounded on a portion or on all of their peripheries by water bearing rocks labelled 'aquifers'. The aquifer may be so large compared to the reservoir that for all practical purposes the aquifer appears infinite. From another perspective, the reverse is also true, i.e. that the aquifer has small and 
negligible effects on reservoir performance. The aquifer may be entirely bounded by an impermeable rock so that the reservoir and aquifer together form a closed (volumetric) unit [5][7].

An alternative is that the reservoir may be replenished by surface water. Figure 1-5 shows a schematic of a water drive reservoir.

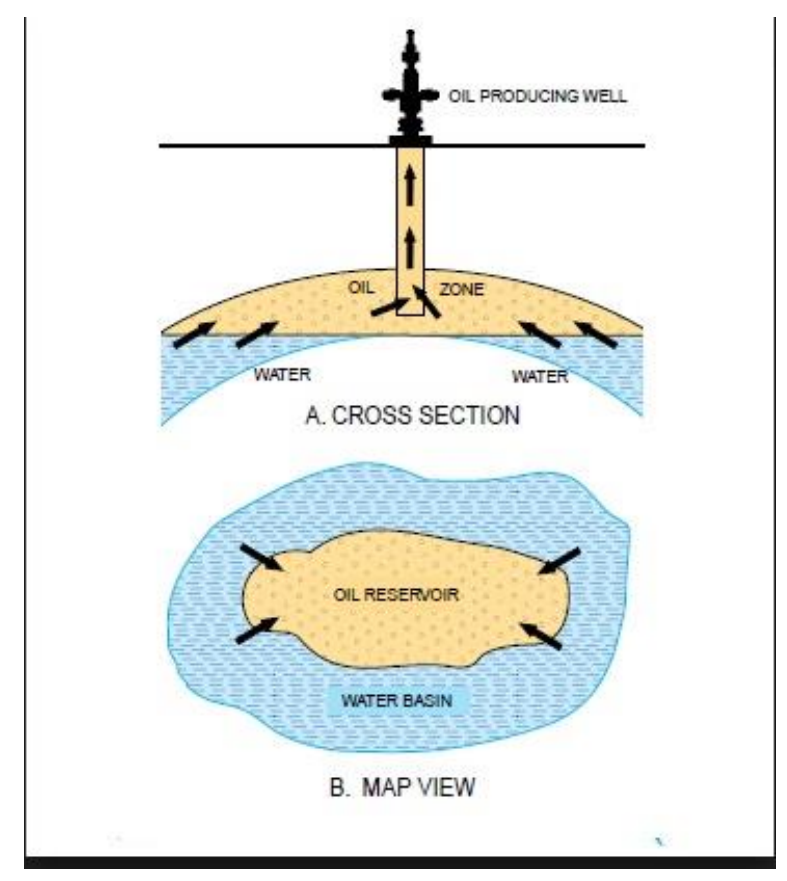

Figure 1-5: Schematic of a water drive mechanism (Artesian water drive) [7].

Figure 1-6 shows the two potential sources of water influx into a reservoir, by edge water or bottom water. Bottom water contact is directly beneath the oil-water contact depth in one continuous surface contact, while edge water occurs off the flanks of the structure at more than one edge surface of the oil reservoir. 


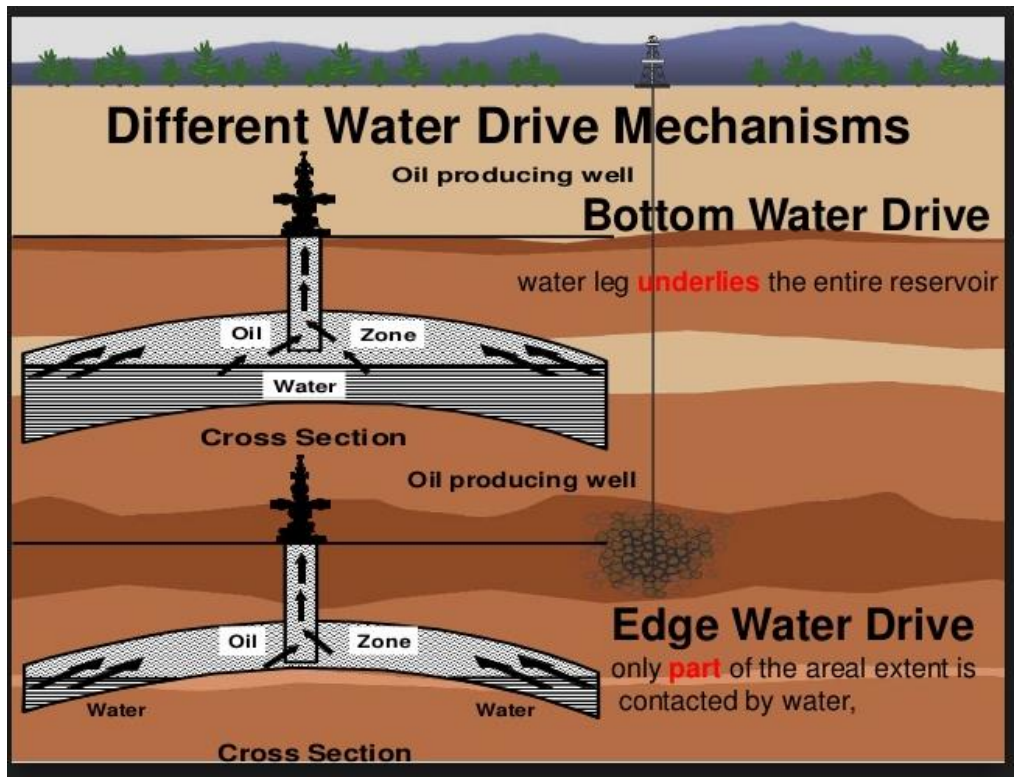

Figure 1-6: Types of water influx common in the water drive [7].

Regardless of the source of water, the water drive is the result of water moving into the pore spaces originally occupied by oil, replacing the oil and displacing it into the producing wells.

Reservoir Pressure: The pressure decline in the reservoir is gradual, as shown in Figure 17.

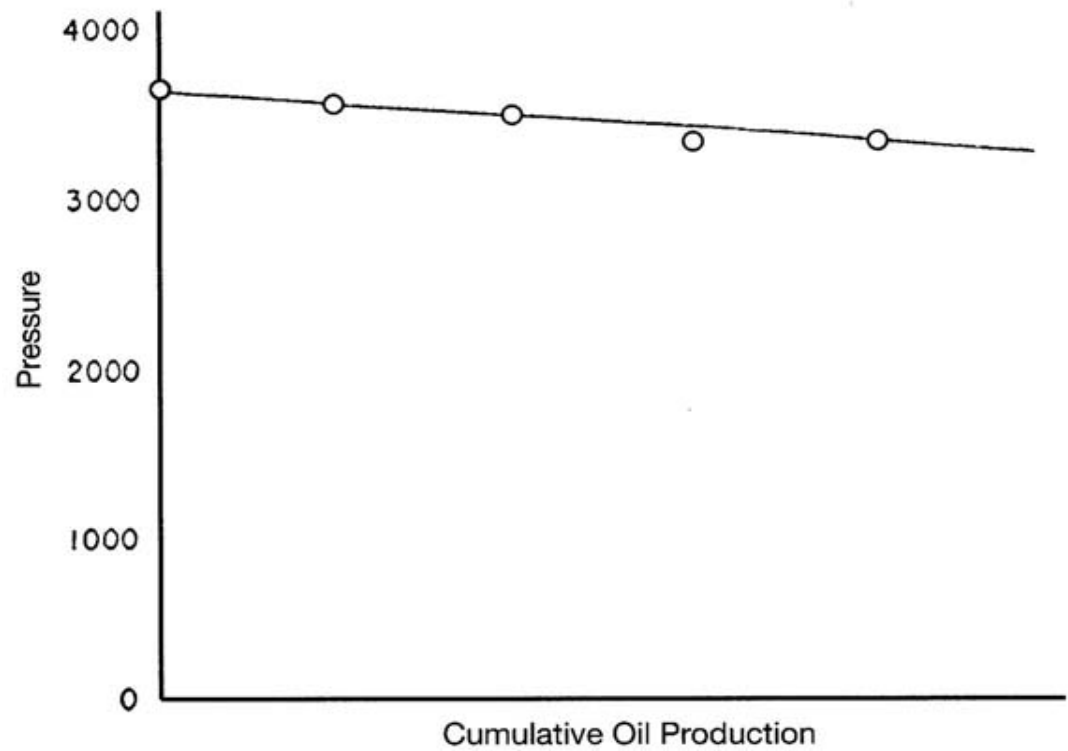

Figure 1-7: Reservoir pressure decline in water drive reservoir [5]. 
The reason for a gradual drop in reservoir pressure is that for each unit of oil and gas withdrawn from the reservoir, there is an equal or almost equal amount of water replacing these fluids and/or encroaching into the oil zone. It should be understood that total reservoir fluid withdrawals are the criteria for maintenance of reservoir pressure.

Since usually the principal economic benefit is from oil production alone, if the withdrawals of water and gas can be minimised, then the withdrawal of oil from the reservoir can be maximised with minimum pressure decline. In this case, it can be said that it is extremely important to reduce water and gas production to an absolute minimum. This requirement can usually be accomplished by shutting down wells producing large quantities of these fluids and, where possible, transferring their production quota to other wells producing with lower water-oil or gas-oil ratios [5][7].

Water Production: Initially, excess water production occurs in structurally low wells. This is one of the characteristics of a water-drive reservoir, and provided the water is encroaching in a uniform manner, nothing should be done to restrict this encroachment, as the encroaching water will probably provide the most efficient displacing mechanism possible.

If the reservoir has several lenses of very high permeability, the water will move through more permeable zones. In such cases, it will be more economical to perform remedial operations that can preferentially shut off the water producing permeable zone. Note that in many cases oil recovered from a structurally low well can eventually be recovered from wells more highly located on the same structure. Therefore, careful consideration to well planning and well control should be taken at all times, otherwise the considerable expense involved in remedial work may prove needless.

Gas-Oil Ratio: Mostly, there are little changes in the gas-oil ratio during the life of the reservoir. This is usually true when the reservoir has no initial free gas cap. Pressure will be maintained as a result of water encroachment, which results in a limited amount of gas that will be out of the solution.

Ultimate Oil Recovery: Ultimate recovery from a water-drive reservoir is generally larger compared to other producing mechanisms. Recovery ratios finally rely upon the efficiency of the 
flushing action of the water as it displaces the oil. The more the reservoir has heterogeneous features, the higher the probability the field will exhibit a decrease in the final recovery ratio. This is due to the uneven advance of the displacing water [1][8].

\subsubsection{Gravity Drainage Drive}

This drive mechanism occurs in petroleum reservoirs as a result of differences in the densities of the reservoir fluids. Gravitational force can simply be illustrated by placing crude oil and water into a jar and agitating the content. When at rest, the denser fluid (normally water) will be at the bottom of the jar, while the less dense fluid (normally oil) will remain on top of the water. This separation noticed is because of gravitational force acting on both fluids.

In petroleum reservoirs, the same has been noticed for the entire component in the reservoir, i.e. the gas content is placed on top of the oil, and the oil placed on top of the water in the reservoir. Figure 1-8. shows the initial fluid distribution in an oil reservoir.

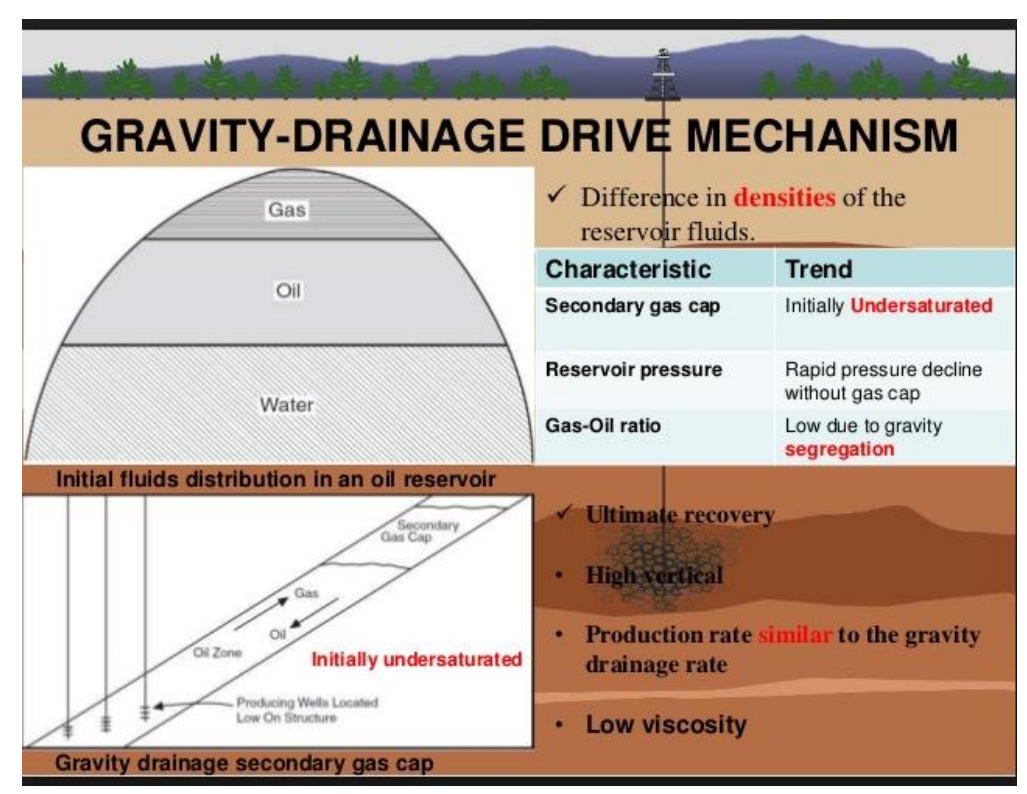

Figure 1-8: Initial fluid distribution in an oil reservoir [5].

Based on the long period of time involved in the petroleum accumulation and migration processes, it is assumed that the reservoir fluids are in equilibrium. If the reservoir fluids are in equilibrium, the gasoil and oil-water contacts should essentially be horizontal (although it is often the case in the field that it is difficult to determine accurately the reservoir fluid contact). Most data from oil fields does however indicate that reservoir fluid contacts are essentially horizontal [6]. 
Reservoir Pressure: Gravity drainage mechanisms shows rapid reservoir pressure decline and different rates of pressure decline depending on the amount of gas conservation. Where the gas is conserved and reservoir pressure is maintained, the reservoir will be operating under a combined gas-cap drive and gravity-drainage mechanism.

Gas-Oil Ratio: A low gas-oil ratio from structurally low wells is expected, mainly due to the migration of evolved gas up-structure, after the gravitational segregation of the fluids. The structurally high wells will experience an increasing gas-oil ratio as a result of the up-structure migration of the gas released from the petroleum crude.

Secondary Gas Cap: There is the formation of a secondary gas cap in a reservoir that is initially under saturated. The gravity-drainage mechanism does not operate until the reservoir pressure declines below the saturation pressure, since above saturation pressure there is no free gas in the reservoir.

Water Production: For gravity-drainage drives, little or no water production is expected.

Ultimate Oil Recovery: Ultimate recovery from gravity-drainage reservoirs varies widely, mainly and primarily to the extent of depletion by gravity drainage alone. Recovery can be very high where gravity drainage is good, if production is restricted to the maximum advantage of the gravitational forces. Reports have been made of over $80 \%$ efficiency rates in the recovery of initial oil in place [5][6].

\subsubsection{Combination Drive}

This driving mechanism involves both water and free gas as sources of displacing actions, sweeping the oil towards the producing wells. Hence, the name combination-drive mechanism, with two main driving forces present in combination, but usually in differing drive strengths:

- Depletion drive and weak water drive

- Depletion drive with a small gas cap and a weak water drive.

Gravity segregation will be a determinant in any of the noted drive mechanisms. A schematic diagram for a combination drive reservoir is shown in Figure 1-9. 


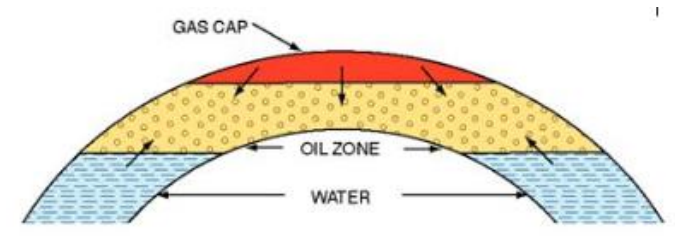

A. CROSS SECTION

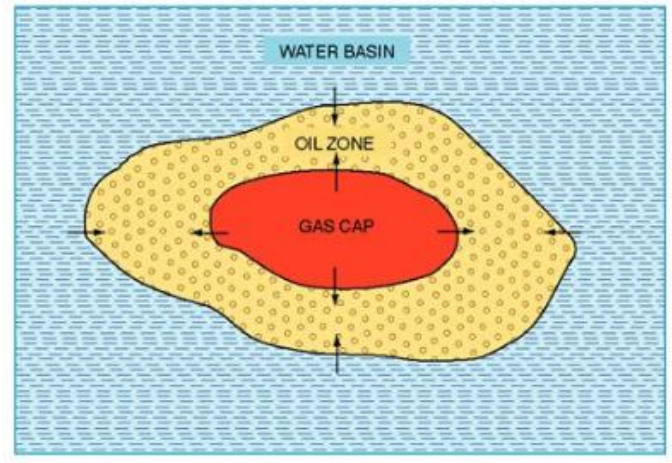

B. MAP VIEW

Figure 1-9: Combination-drive Reservoir [5].

\subsubsection{Factors Influencing Combination-Drive Reservoir}

Relatively rapid pressure decline: Water encroachment and/or external gas-cap expansion are often insufficient to maintain reservoir pressures.

Water encroaching slowly into the lower part of the reservoir: Down dip structurally producing wells will exhibit slowly increasing water producing rates.

If a small gas cap is present, the structurally high wells will exhibit continually increasing gas-oil ratios, as the gas cap expansion occurred. It is possible that the gas cap will shrink due to production of excess free gas, in which case the structurally high wells will exhibit a decreasing gas-oil ratio. Large amounts of in situ oil can be lost from production as a result of a shrinking gas cap, and so this situation should be avoided whenever possible [5].

A substantial percentage of the total oil recovery may be due to the depletion-drive mechanism. The gas-oil ratio of structurally low wells will increase due to evolution of solution gas throughout the reservoir, as pressure is reduced.

The final recovery from combination-drive reservoirs generally exceeds the recovery ratios from depletion-drive reservoirs, but can be expected to be less than recovery from water-drive or gas-cap-drive reservoirs. Final recovery will be heavily influenced to the extent it becomes possible to minimise the influence of recovery by depletion drive. In most combination-drive 
reservoirs, it will be desirable to institute pressure maintenance either by gas injection, water injection, or by both gas and water injection, depending upon the availability and the cost of the fluids [5].

\subsection{Secondary Oil Recovery}

Primary recovery techniques normally remove no more than about $30 \%$ of the oil in a reservoir. Petroleum engineers have long realised that a significant fraction of the remaining oil can be forced out by fluid injection. Flow out of the reservoir continues under the influence of a number of natural factors, such as gravity, pressure of surrounding water, and gas pressure. This flow can be continued and extended by means of pumping [11].

The process of fluid injection involves the drilling of a second hole into the reservoir at some distance from the first hole through which oil is produced. Some gas or liquid is then pumped down into the second hole, increasing pressure on the oil remaining in the reservoir. The increased pressure forces more oil out of the reservoir and into the recovery pipe.

Secondary recovery methods are thus used when the natural or primary recovery method of extracting oil from the reservoir is exhausted. That is, the reservoir pressure or energy from the reservoir, which drives oil towards the wellheads of producing wells, is no longer capable of moving oil towards the direction of the well. The single most common secondary recovery technique is water flooding. When water is pumped into the second well, it diffuses out into the oil reservoir and tends to displace oil from the particles to which it is absorbed.

Water flooding was used as early as 1900. A common practice was to drill a series of wells, some of which were still producing and others of which employed water injection. As soon as the old producers became wet, they were converted to water injection wells and another group of producer wells were drilled. The process was repeated until all available oil was recovered from the field [49].

Recently, more sophisticated approaches to fluid injection have been developed. The two fluids that have been used most extensively in these approaches are liquid hydrocarbons and carbon dioxide. The principle behind liquid hydrocarbons is to find a material that can mix more completely with oil and then 
allow the oil-mixture that is formed to be driven more easily out of the reservoir, into the recovery pipe, and with enhanced oil recovery. A commonly used hydrocarbon for this process is liquefied petroleum gas (LPG), which is completely miscible with oil [49].

Since LPG is economically expensive, only a small volume is actually used. The LPG is pumped into the reservoir to be followed by a different "pusher gas" The pusher gas, often methane, is considerably less expensive and can be used in larger volumes. The pusher gas forces LPG into the reservoir where the LPG then mixes with residual oil. The mixture is more mobile than the original hydrocarbon components and can be displaced by the 'pusher gas'.

This type of recovery process has worked well in the laboratory, but less well in actual practice. In field conditions, the LPG has a tendency to get lost in the reservoir to an extent that it does not effectively dissolve into and ultimately improve the removal of the residual oil. The heterogeneous quality of most reservoirs disallows, or at least poses many challenges, for the use of LPG dispersal and pusher techniques. Another secondary recovery technique is to use an actual fluid in the injection stage to help extract the remaining oil from a reservoir.

When these techniques are used immediately after primary recovery, they are known as secondary recovery techniques. If such injection methods are employed a second time after (primarily water) fluid injection has been tried, the methods are often referred to as tertiary recovery techniques. A whole set of recovery techniques can be called by different names, depending on the stage at which they are used. Secondary methods by water injection can be referred to as Improved Oil Recovery (IOR). However, it is becoming more common today to refer to any method for removing the residual oil from a reservoir as an Enhanced Oil Recovery (EOR) technique.

One EOR technique for removing residual oil from a reservoir makes use of surfactants. A surfactant is a substance whose molecules are attracted to water at one (hydrophilic) end and oil at the other (oleophilic) end of the molecule. The most familiar surfactants are the soaps and detergents found in every home [49].

If surfactants are injected into an oil reservoir, they will form emulsions between the oil and water inside the reservoir. Oil is usually washed off particles of rock in the reservoir the way grease is washed off a pan by a household detergent. The emulsion that is formed is then pushed through the reservoir and out of the producer pipe by a flood of water pushed down the injection pipe. 
The surfactant method works well in the laboratory, although not fully successfully in the field. Surfactants tend to adsorb on rock particles and get left behind as the water pushes forward. Methods for overcoming this problem are now being explored [11].

One of the fundamental problems with recovering residual oil in a reservoir is that oil droplets often have difficulty in squeezing through the small openings between adjacent rock particles. The use of surfactants is one way of helping the oil particles pass through such openings more easily. Another approach is to increase the temperature of the oil inside the reservoir, thereby reducing its viscosity (tendency to flow). Oil becomes less viscous and can be produced more easily, with the same pressure then forcing what was immobile oil through pores in the reservoir.

As a first application for this method, the steam soak method, was first used in Venezuela in 1959. In this method, steam is injected into one part of the reservoir and the producer will be closed. After a few days, the well will be reopened, and the oil then flows out. This process is repeated a few times before a change is made in the method and steam is pumped in continuously while the producer remains open. Steam injection works especially well with heavy oils that are not easily displaced by other secondary recovery techniques. The technique is now used commercially in a number of fields, primarily in Venezuela and California [52].

A dramatic form of secondary recovery is in situ combustion. The principle involved is simple and involves a portion of the oil in the reservoir being set on fire. The heat from this combustion then warms the remaining residual oil and reduces its viscosity, forcing it up the producer. In practice, the fire can sometimes be made to ignite spontaneously simply by pumping air down the injection well. In some cases, however, the oil must actually be ignited at the bottom of the well. The temperature produced in this process may reach $650-1200^{\circ} \mathrm{F}\left(350-650^{\circ} \mathrm{C}\right)$ and the region of burning oil may creep through the rock at a speed of $1-12$ in $(3-30 \mathrm{~cm})$ per day. As the fire continues, air, and usually water, is continually pumped into the injection well to keep the combustion zone moving. Under the best of circumstances, in situ combustion has recovered up to $50 \%$ of the oil remaining in a reservoir.

In general, each recovery method is suitable for particular kinds of reservoirs. Oil viscosity, rock porosity, depth of the reservoir, and the amount of oil remaining in the reservoir are all factors that 
determine which method to use. To date, however, the only method that has proved to be both economic and practical in actual field situations is steam injection [1][3][4][5].

\subsubsection{Water Flooding/Water Injection}

Water flooding is one of the methods/techniques labelled a secondary recovery process. Water is injected into the reservoir to improve the lost energy from the initial stored energy of the reservoir; as water is forced through the injector wells into the reservoir, the oil in the reservoir is displaced towards the producing well, along finally with injected water as it reaches the producer well and is recovered as a by-product. With time, more water is produced (the water cut rises), until the water ratio rises so high that the flooding process is stopped or discontinued for further recovery processes to be assessed and perhaps adopted.

While the average primary recovery efficiency is around $15 \%$ of the oil in place, the water flooding processes can raise the recovery substantially, by another $15-20 \%$ of the original oil in place. However, it must be noted that this still leaves the majority, and a huge amount of oil left in the reservoir.

The overall objective of water flooding is to increase the mobility of the oil, which is the effective permeability of the rock to the oil phase, divided by the viscosity of the oil. This is expressed as follows:

$$
\lambda=\frac{K}{\mu}
$$

In equation $1.1, \lambda$ is the mobility of the oil, $\mu$ is the fluid viscosity in centipoises (cp) and $k$ is the effective permeability of the reservoir rock, in millidarcies (md).

Furthermore, the mobility ratio $(\mathrm{M})$ is the mobility of the water divided by the mobility of oil, i.e.

$$
\mathrm{M}=\frac{K_{r w} \mu_{o}}{K_{r o} \mu_{w}}
$$

In equation $1.2, K_{\text {ro }}$ is the relative permeability of oil, $\mu_{\mathrm{o}}$ is the viscosity of oil, $\mu_{\mathrm{w}}$ is the viscosity of water, and $K_{\mathrm{rw}}$ is the relative permeability of water.

A value of $\mathrm{M}<1$ is indicative of a favourable displacement as oil moves faster than water; $M=1$ also indicates a favourable displacement as both the oil and water move at equal speed and $\mathrm{M}>1$ indicates an unfavourable displacement as the water moves faster than oil. The advantage of this method is that there is no change in the state of the recovered oil. However, this technique may be too expensive if insufficient 
formation water is readily available, especially during the early years of an oil field, and thus some other fluid is needed for requiring external pressure maintenance [9].

A schematic of a water flood operation is shown in Figure 1-10.

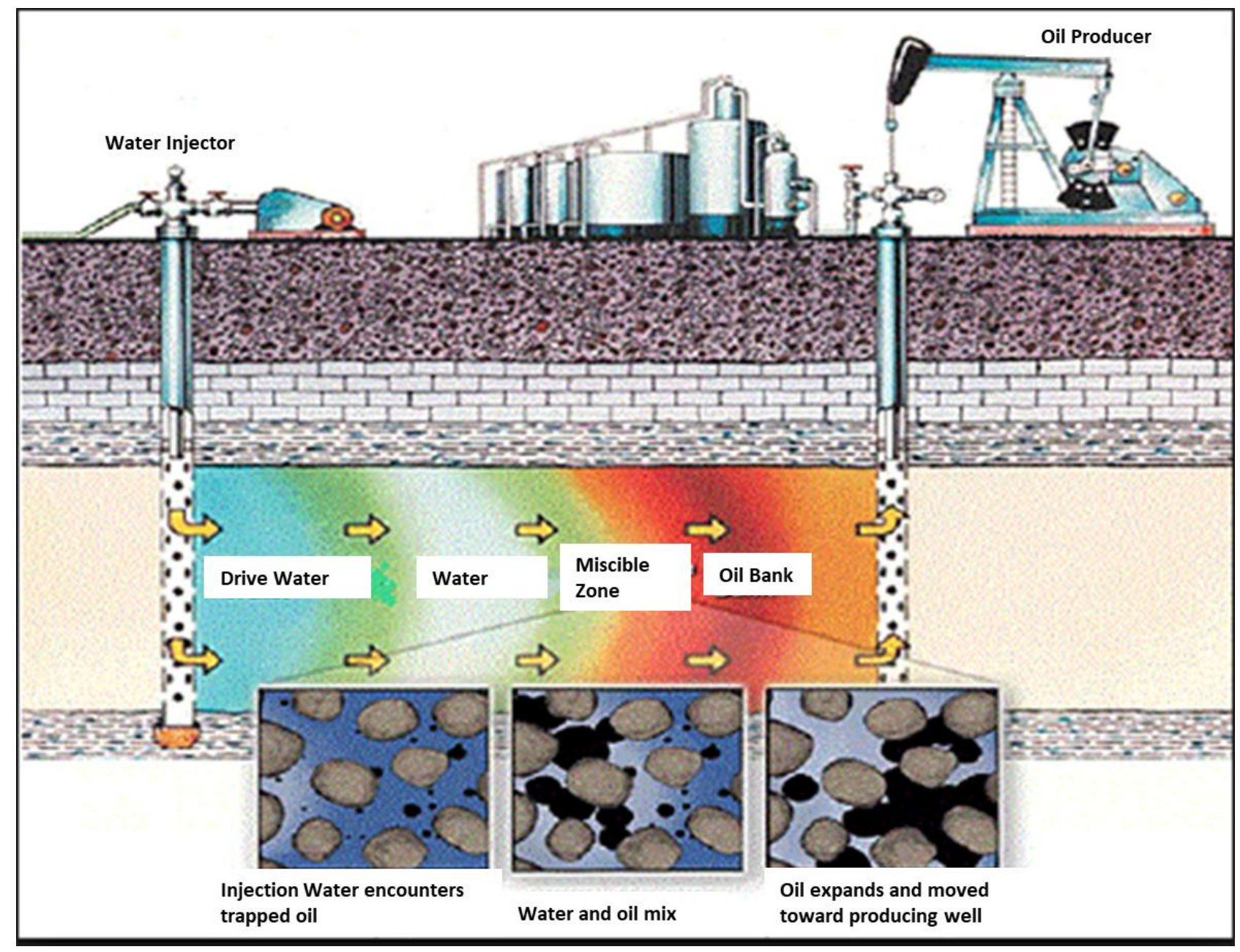

Figure 1-10: Schematic of a Typical Water Flood Operation Method [6].

\subsubsection{Gas Displacement Process}

Secondary production methods are employed to increase the production of oil basically by boosting the depleted pressure in a formation. As the oil, or natural gas, in a formation is produced, the hydrocarbons remaining in the reservoir may become trapped because the pressure in the formation has 
reduced, causing the production to be either dramatically slow or to stop altogether. Gas displacement recovery methods involve the usage of gases such as carbon dioxide $\left(\mathrm{CO}_{2}\right)$, nitrogen and LPG as sources of gases injected into the reservoir. Perhaps the most effective gas fluid now available for injection appears to be carbon dioxide.

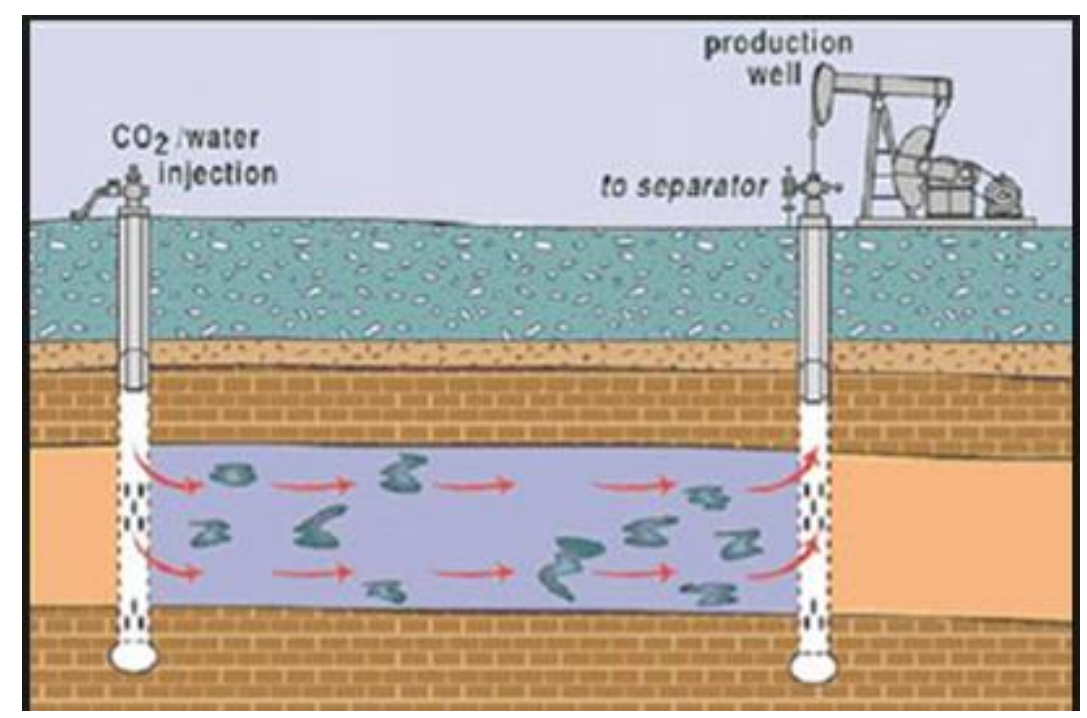

Figure 1-11: A Typical $\mathrm{CO}_{2}$ Gas Injection Method [5]

A typical gas injection schematic diagram is shown in Figure 1-11. The carbon dioxide process has been shown to be effective in displacing oil after water flooding has already been used and only $25 \%$ of the oil in a reservoir still remains.

A mixture of carbon dioxide and water can also be pumped down into the reservoir to be followed by an injection of pure water. The pure water injection works to drive the carbon dioxide-water mixture through the reservoir. As the partially miscible carbon dioxide comes into contact with the oil, it dissolves in the oil, causing the oil to expand and break loose from the surrounding rock. The oil-carbon dioxidewater mixture is then pumped out of the recovery pipe where the carbon dioxide is removed from the mixture and re-used in following secondary recovery phase.

However, in many cases, carbon dioxide is more efficiently used when reservoirs contain a larger fraction of residual oil. The gas is injected into the gas-cap, compared to water flooding injected directly into the oil zone. 


\subsection{Tertiary Recovery /Enhanced Oil Recovery Method}

Commonly, a larger reservoir will go through three different phases throughout its lifetime, namely the primary recovery, secondary recovery and tertiary recovery phases. The aim of all these phases is to maximise the production of oil during its life. As has been emphasised, primary recovery recovers only a small fraction of the original oil in place, secondary recovery increases the productivity to a third or more, while tertiary recovery (EOR) can help to recover over half of the original oil in place from the reservoir depending on the particular EOR adopted.

There are many different methods of enhanced oil recovery methods that can be adopted. Before the best-fit method is chosen, reservoir core samples are extensively tested. The best results will determine the best-fit method to be adopted. Such methods include Thermal EOR, Miscible EOR, Chemical EOR and others [7].

The tertiary recovery method introduces fluids that impact the recovery process since the fluid reduces the viscosity and improves the rate of flow towards the producing well head(s). The tertiary fluids can consist of gases that are miscible with oil (mostly carbon dioxide), steam, air or oxygen, polymer solutions, gels, surfactant-polymer formulations, alkaline-surfactant-polymer formulations and microorganism formulations.

\subsubsection{Thermally Enhanced Oil Recovery}

This process involves the injection of steam into the well. Hot water or steam reduces the viscosity of the oil, which enables the oil to flow more easily towards the wellhead. Various types of steam injection are applied, such as cyclic steam injection, which involves steam being pumped through the same well as that in which the oil will be produced. Continuous steam injection involves steam injected through a particular well continuously, while the oil is produced through another producing well. There is also hot water injection, steam assisted gravity drainage (SAGD) using horizontal wells, in-situ combustion and fire flooding. This process involves the injection of air or oxygen, i.e. the oxidation of the oil in place. The following reactions will generally occur:

- Heat is being produced to reduce the viscosity of the oil in the reservoir 
- Higher molecular hydrocarbons may be cracked into smaller molecules

- Vaporisation of some of the lighter hydrocarbons to make the oil components more miscible and also by the formation of gas, to help displace oil.

A typical schematic diagram for thermal recovery method technique is shown in Figure 1-12.

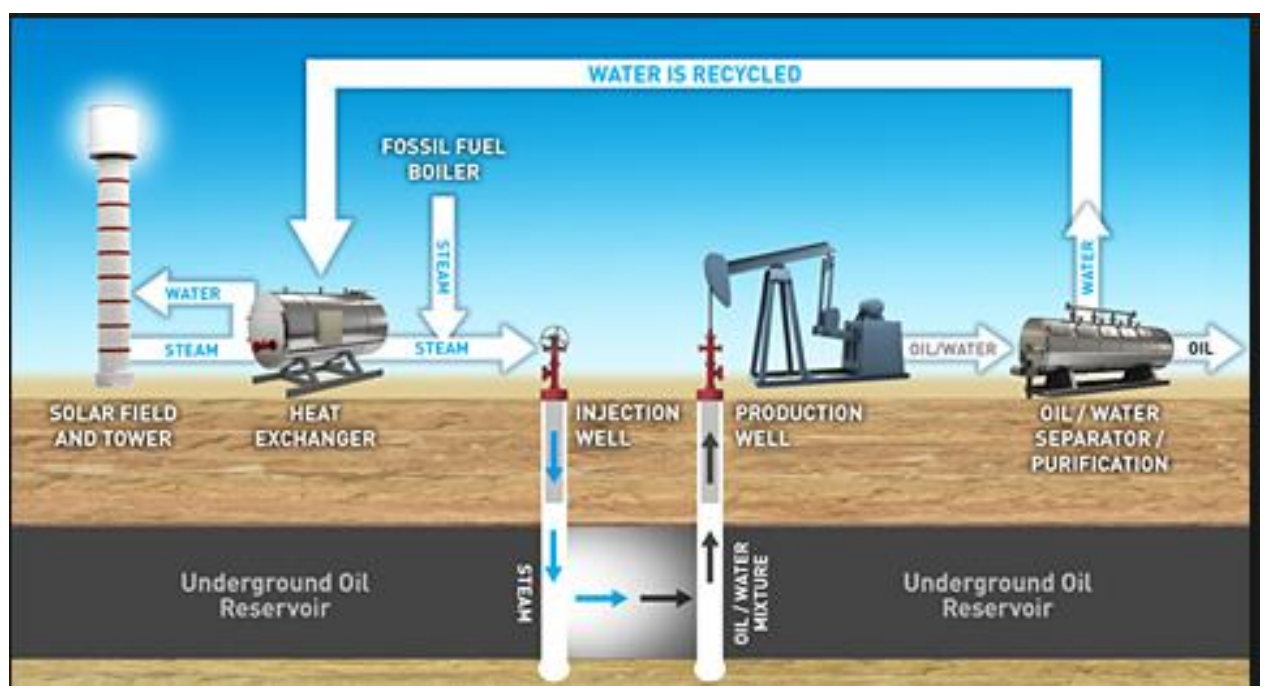

Figure 1-12: Typical Thermal Recovery Method [7].

\subsubsection{Miscible Enhanced Oil Recovery}

Carbon dioxide can form a gas, liquid, solid and supercritical fluid at a steady change in pressure and temperature. At or above the critical point, $\mathrm{CO}_{2}$ can maintain the properties of a gas and still have the density of a liquid. The miscible Enhanced Oil Recovery process involves the use of supercritical $\mathrm{CO}_{2}$ to displace the oil from a depleted oil reservoir with suitable characteristics, typically light oil.

The injected miscible $\mathrm{CO}_{2}$ mixes thoroughly with the oil within the reservoir such that the interfacial tension with these two substances disappears. It also it has the ability to dissolve in, swell and reduce the viscosity of oil.

In deep, high-pressure reservoirs, compressed nitrogen is introduced instead of $\mathrm{CO}_{2}$, and liquefied petroleum gases are used instead of $\mathrm{CO}_{2}$ for miscible oil displacement in some larger reservoirs. A typical schematic diagram for miscible EOR method is shown in Figure 1-13, and miscible EOR Process showing both nitrogen and $\mathrm{CO}_{2}$ injection in Figure 1-14 [9]. 


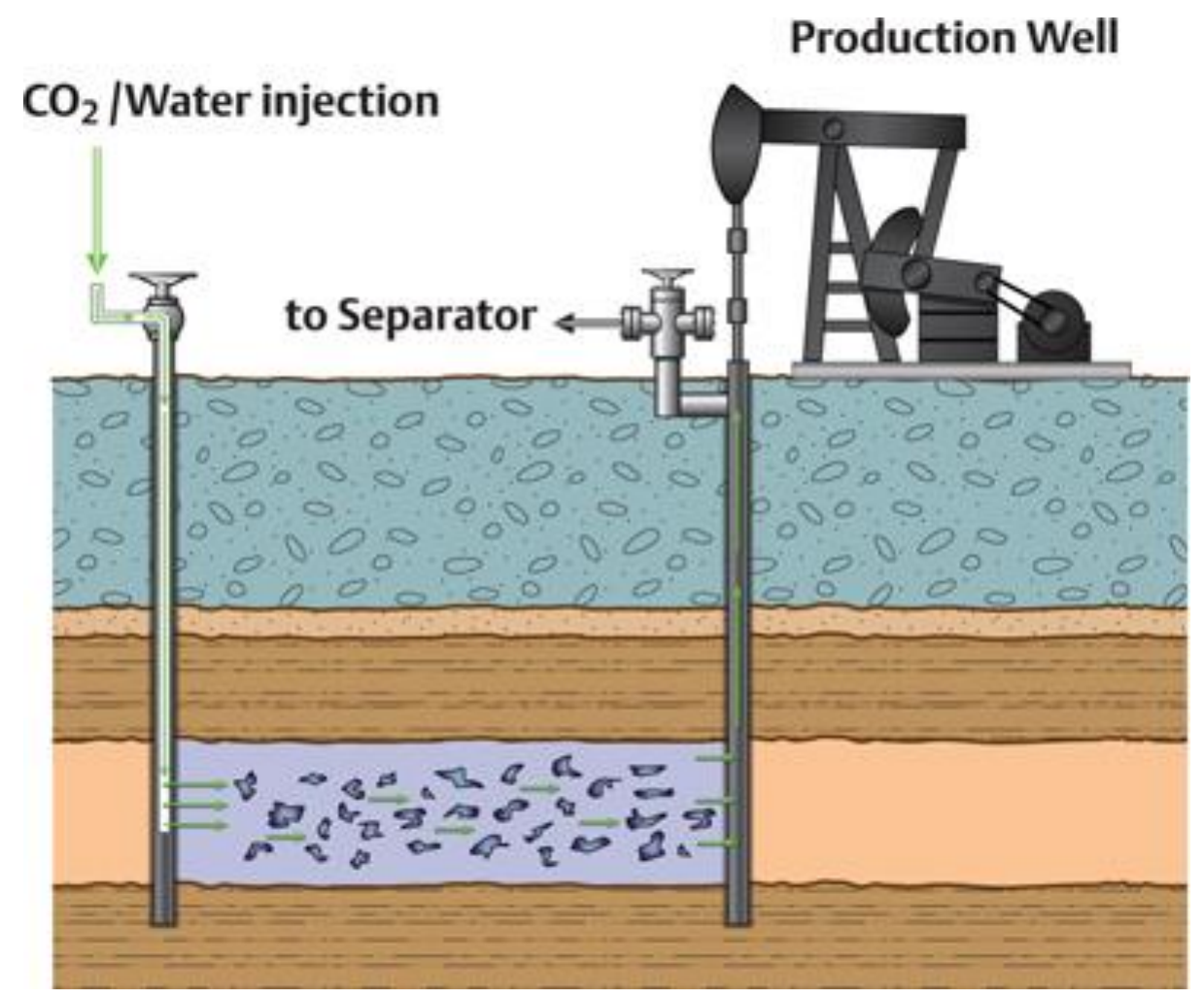

Figure 1-13: Typical Miscible EOR Method. [9]

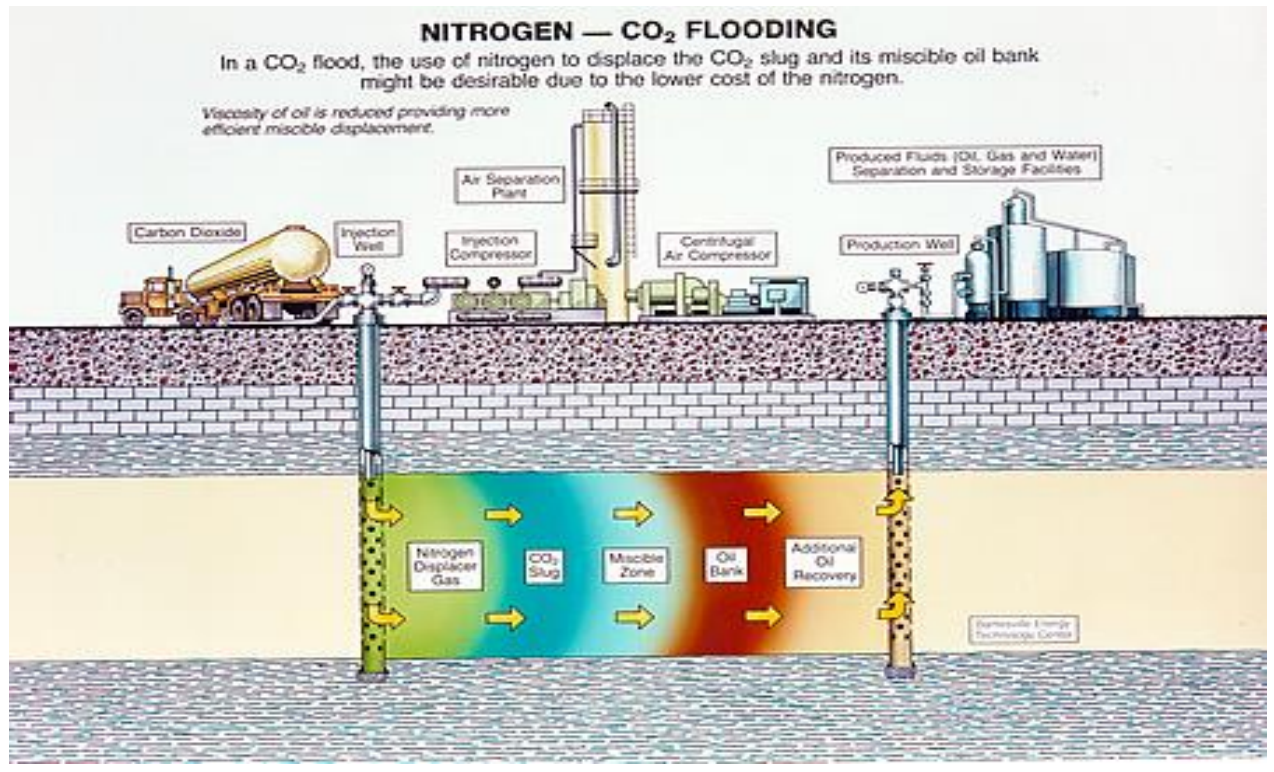

Figure 1-14: Miscible EOR Process showing both Nitrogen and $\mathrm{CO}_{2}$ Injection [9]. 


\subsubsection{Chemically Enhanced Oil Recovery}

This process involves three chemical flooding processes. There is polymer, surfactant-polymer and alkaline-surfactant-polymer (ASP) flooding. The polymer flooding method involves water-soluble polymers, which increase the viscosity of the injected water, and this leads to a more efficient (pistonaction) displacement of moderately viscous oils along the injected polymer front.

The addition of surfactant to the polymer formulation may reduce the oil-water interfacial tension to almost zero, thus displacing the trapped oil. However, the surfactant-polymer flooding method has not been used on a large scale. This method is still at the laboratory test level, where it has very good potential for improving oil recovery.

Alkalines have the ability to convert some acids within the oil to a surfactant molecule that then aids the oil recovery. The alkaline itself can also be useful in reducing surfactant retention in the rock. In any chemical flooding processes, the inclusion of a viscosifier, usually a water-soluble polymer, is a prime necessity to provide an efficient sweep of what expensive chemicals are being injected throughout the reservoir volume. To prevent some thief zone diverting the injected chemicals away from the oil in place, gels are also used to strategically plug fractures or other extremely permeable channels in the reservoir rocks. A typical schematic diagram for EOR chemical method is shown in Figure 1-15. Figure 1-16 shows the flow pattern of injected chemicals into the reservoir rocks [12] [9].

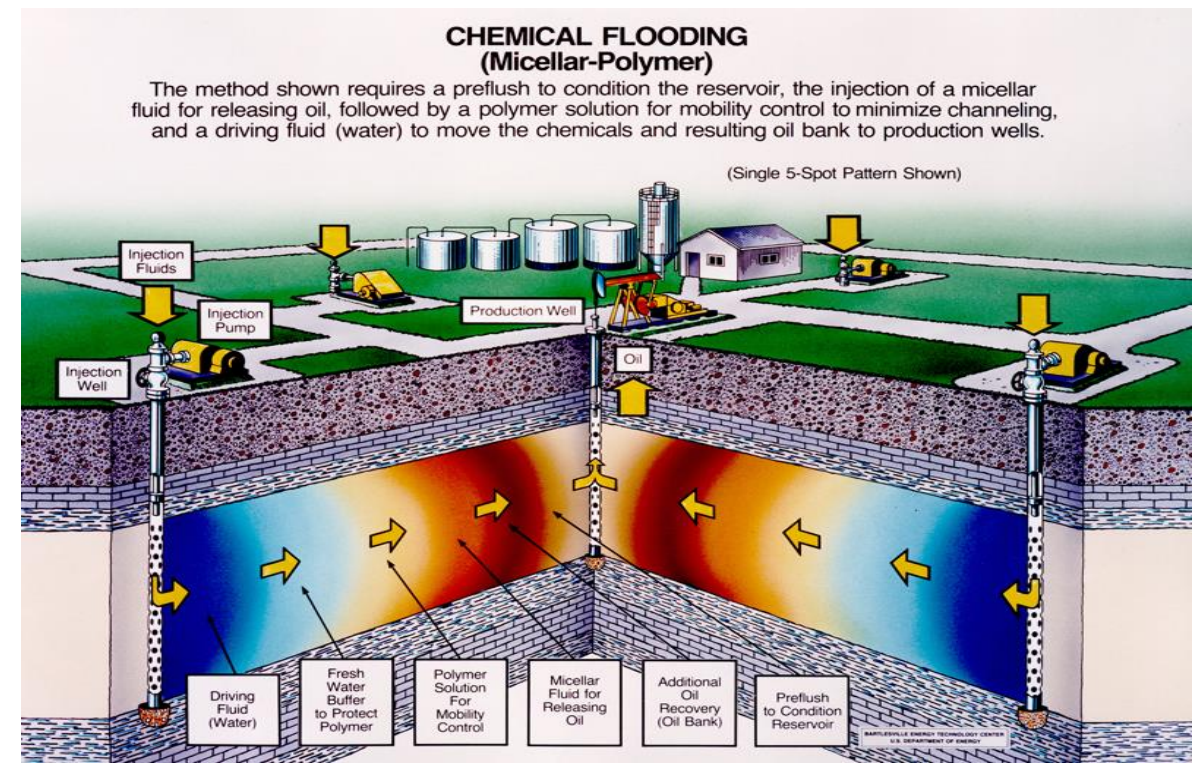

Figure 1-15: A Typical chemical EOR method [12]. 


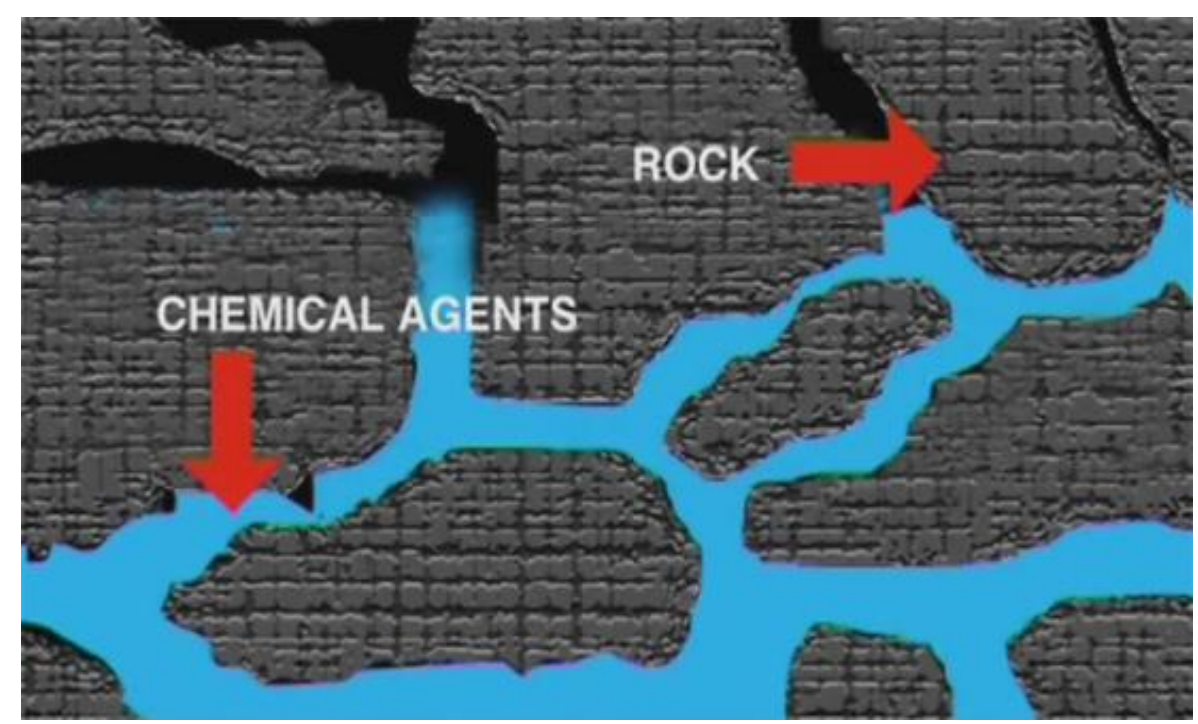

Figure 1-16: Flow pattern of Injected Chemicals into the Reservoir rocks [9].

\subsection{Reservoir Management and Streamline Simulation}

Water-flood and gas displacement studies in reservoir management projects have remained a complex yet valuable means of optimising current resources explored in great detail, ranging from well spacing, volume of fluid injected and economics of both water-flood and gas displacement designs. Advances in computational limits have also made possible the calculation of complex multi-dimensional algorithms on simulation grids that anticipate and bring insight into the behaviour of well-to-reservoir interactions in waterflood studies. However, if it can be relatively simple to investigate well-fluid interactions in homogenous reservoirs, the task becomes considerably complex when anisotropic phenomena are taken into account.

In today's industry, reservoir management also involves investigating production responses, otherwise known as production monitoring and also reservoir responses. When the production responses are used, engineers are less concerned about the grid-based, spatial representation of activity vectors that affect fluid flow, but rather the graphical representation of key variables of production and pressure. Theses graphs are then used to make dynamic inferences on the success of water flood projects. Simulated 
reservoir responses can also provide more detailed strategies that are helpful in making accurate predictions on the expected success of waterflood or gas displacement projects. This is true even when a pilot study has not been carried out; the displaced and displacing fluid can be monitored as fluid fronts and streamlines.

As such, reservoir simulation has gained wide acceptance in reservoir development and management stages. Most simulators are based upon the finite difference method where fully implicit schemes have the capability of solving compressible and slightly compressible fluid systems [66]. However, the degree of heterogeneity among reservoir properties presents ongoing challenges on simulation accuracy. Simulation has also been compromised due to the uncertainty of results compromising business decision and delaying action [66]. In addition, high-end hardware has been required to reduce simulation run times. To narrow the gap created between heterogeneous geological models and flow simulation constraints, streamline simulation technology has been developed, as a more economical alternative to conventional finite difference simulation methods. The Lagrangian particle tracking is a numerical technique for tracking Lagrangian particles within an Eulerian phase and it is commonly used in computational fluid dynamics. In order to describe and visualise the fluid flow, streamline, pathline and streaklines are convenient tools to do so given an external velocity field (q=qx, qy, qz). Streamline are a family of curves and can be traced for any vector field such as q, which represents the velocity obtained from the solution of a set of flow equations. In the case of incompressible fluids, streamlines defined at a single instant do not intersect and cannot begin or end inside the fluid [60].

Streamline technologies introduce a quantity known as time of flight (TOF) compared with conventional Eulerian simulation [9]. Steps in the streamline simulation procedure involve obtaining pressures and velocities from the numerical solution of the governing pressure equation. Re-sampling of the saturations from a grid block to a streamline follows at each pressure update, with gravity corrections and numerical dispersion corrections applied when necessary; transverse flux is managed using the same operator-splitting formulation.

Values added to the original conventional simulation include visualisation of flood fronts, naturally delineating fast and slow flow paths and in turn, provide a natural means for dynamic simulation. Figure 1-17 shows an illustration of the streamline process flow chart [9]. 


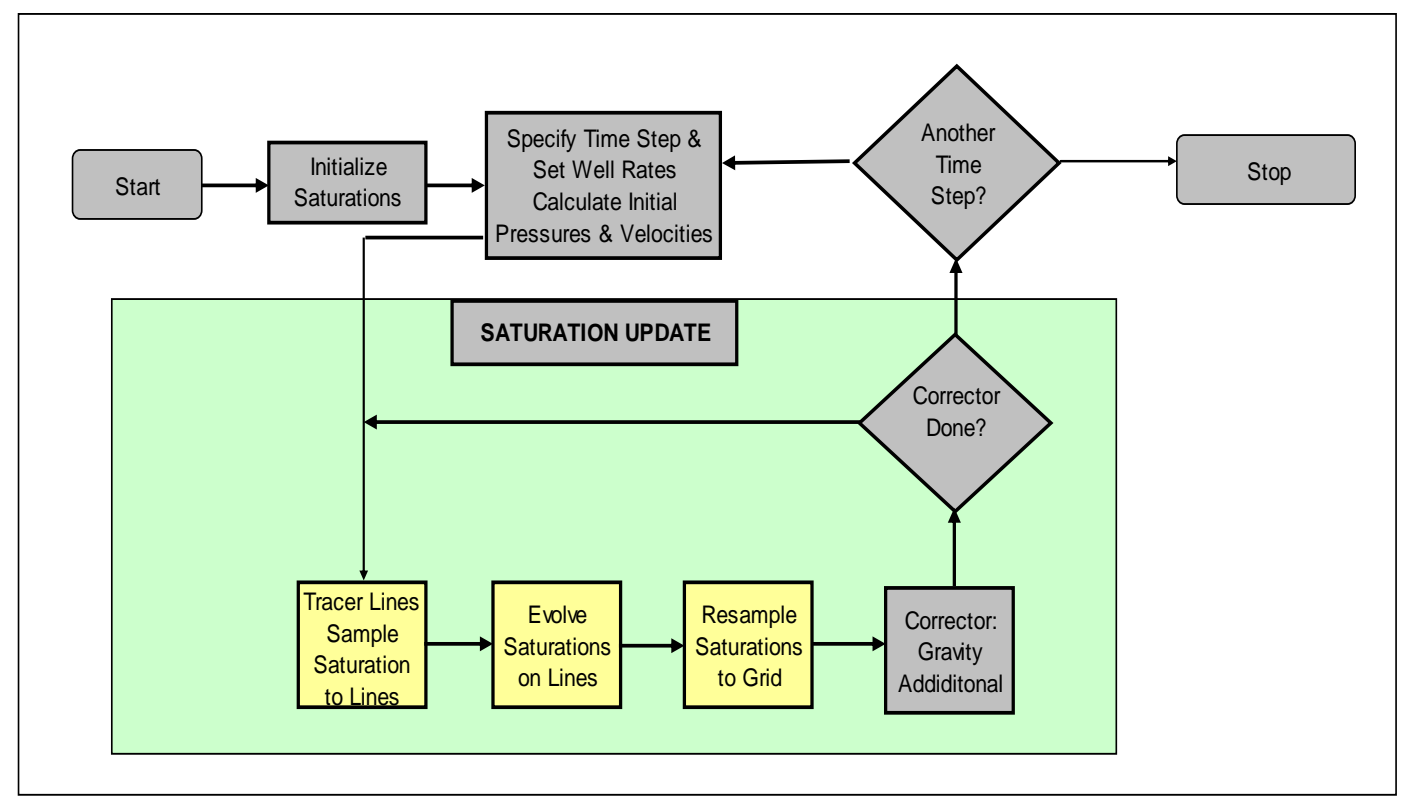

Figure 1-17: Flowchart illustrating steps in streamline simulation (Datta-Gupta, 2007)

\subsection{Problem Description}

The finite-difference method is widely used for solving large-scale multiphase displacement problems, e.g. displacement of oil by water/gas in heterogeneous petroleum reservoirs. In principals, finite difference methods based on IMPES approach suffer from the time step length limiting the CourantFredrich-Levy (CFL) condition. Numerical dispersion, grid orientation effects, small time-step size, and large computation time are normally factors which should be considered in finite difference simulation [19][78]. To overcome these issues, in streamline methods fluid is transported along the streamlines instead of the finite difference grid. Streamline simulation is an ideal reservoir management tool for mature water floods as it is easy to identify non-swept zones, evaluate multiple forecast scenarios and obtain novel information such as well pair interactions. Streamline methods are traditionally only applied to incompressible flow processes and are not yet fully implemented in compositional models [25]. In this work, a three-dimensional black oil and compositional streamline model is developed and integrated with an existing finite difference simulator to study water flooding, Water Alternate Gas and gas injections in a quarter five spot pattern. The 3-D multi-component material balance equation is decomposed into 1-D 
equations along the streamlines using the streamline time of flight as the spatial coordinate. The pressure field is solved in the conventional finite difference manner and streamlines are traced from injector to producer. Gravity effects are added using the operator splitting technique to account for the gravity segregation due to density differences. This simulator can handle the formation and flow of the fourth phase (third hydrocarbon phase). The streamline module is parallelised by distributing streamlines among different processors because computations along any streamline are independent of other streamlines and no communication is required.

Streamline simulation is ideally suited for modelling large heterogeneous multi-well systems dominated by convection. While Numerical Simulation methods can account for the convective terms, this is the domain where Numerical Simulation methods fail in practice. Conversely, the streamline method is not well suited to complex physics displacements (high compressibility, capillary effects, complicated phase behaviour). There is no numerical method that can solve the governing equation for all cases efficiently. As the gas displacement processes are one of the critical areas that have not been solved yet by streamline simulation, mainly because of the compressibility issue, this project will focus on studying streamline applications for gas displacement processes in more details. In this thesis it will attempted to produce guidelines on how to use Streamline Simulation for Gas Displacement processes for improved oil recovery.

Several trials have been attempted prior to this work addressing the compressibility issues [18][19]. Crane et al (2000), proposed 1-D fully implicit solutions of FD simulation are used to solve for pressure and fluid compositions together along each streamline, and account for the changes of the phase behaviour that depend on the changes in pressure. It was tested and compared against FD compositional simulator using SPE 9 Model; this work on SL showed that it is significantly faster and requires less memory [19]. Theile et al [59] developed a 3-D 3-phase compositional simulator for $\mathrm{CO}_{2}$ injection accounting for both gravity and capillary effects using orthogonal projection. This proposed approach enables the use of a larger time step compared with conventional operator splitting. The results showed a good agreement and more efficiency in comparison to the FD compositional simulator [59].

The fast-marching method technique is also be used to calculate a diffusive time of flight at every location within the domain. The diffusive time directly related to pressure from distribution, which can be solved by Eikonel equation. As a fast way, this pressure front distribution can be determined in a single non- 
iterative calculation and once this pressure fronts determined spatially, a pseudo steady pressure calculation within the moving from may be applied to determine the pressure depletion and well rates. Zahng ea al in (2013) used this fast marching technique to solve Eikonol equation for complex grids which allows the rapid approximation of reservoir simulation results without the need of complex flow simulation calculations. [69]

A fast and novel workflow for compositional simulation for unconventional reservoir was proposed in 2017. The approach was based on high frequency asymptotic solution of the diffusivity equation and was mainly tested for unconventional reservoirs. In this approach, high frequency equation leads to the Eikonal equation which is solved for 'diffusive time of flight' (DTOF) which governs distribution of the pressure front in the reservoir. This workflow consists of two decoupled steps: calculation of the DTOF using the Fast Marching Method (FMM) and fully-implicit compositional simulation using DTOF as a spatial coordinate. The results showed significant reduction in computational efficiency by reducing 3D compositional flow to $1 \mathrm{D}$ equation using DTOF as spatial coordinate. The savings in computation time increases significantly with grid refinement and for high-resolution models [70].

\subsection{Procedures Employed}

In the first part of this research, we build several hypothetical fine grid numerical simulation models for black oil reservoirs under water flooding, Water Alternate Gas and Gas Injection. We generate Finite Difference and Streamline Simulation models for the same reservoirs, compare the predictions and performance of both models and quantify the differences. The outcome of this work was published on SPE 169703-MS, which was presented at SPE EOR Conference held in Muscat - Oman, March 31, to April 2nd 2014.

In the second part of the research, several test cases have been generated for gas displacement using the numerical models. Then, several streamlines models been built with the same assumptions of the numerical model for gas displacement. Comparisons between FD and SL models have been done and generated several differences in terms of time, displacement, production performance and recovery factors. These differences were used to derive some guidelines for the use of streamline simulation in gas 
displacement processes. The outcome of this work was published on SPE 172261-MS, presented at the SPE Annual Caspian Technical Conference and Exhibition held in Astana, Kazakhstan, 12-14 November 2014 and SPE 178031, presented at the SPE Annual Technical Symposium and Exhibition held in AlKhobar, Saudi Arabia Section. In addition to the previously mentioned SPE papers published out of this work, it is also peer reviewed and published in the International Journal of Multiphysics. "12 International Conference of Multiphysics held in Beijing, China on 14-15 December 2017".

\subsection{Expected Results}

The expected results from this research project include:

1. Testing the approach of streamline simulation for gas displacement processes

2. Determining how far the streamline simulation is from finite difference modelling

3. Quantifying and minimising the gap between the two simulation techniques

4. Providing guidelines for streamline simulation modelling for gas displacement processes.

Streamline simulation technology has seen rapid progress over the past two decades, in terms of both its engineering application and its mathematical basics. The improvement of this technology has been driven by its efficiency over conventional finite-difference simulation, especially for:

- Flow simulation of large multimillion-cell geologic/static models.

- High-resolution geological models characterised by heterogeneous spatial distributions of static properties.

- The screening calculations for a large number of realisations of these models.

- The speed and versatility

- Flow visualisation as it allows pattern surveillance of well-pair interactions.

This effectiveness of streamline technology has reduced the widening gap between geological modelling and flow simulation. For a sensible reservoir management practice, the impact of the unknown elements of the static model on fluid flow and transport must be understood and quantified. This is possible with increased model resolution, which comes with the increased acknowledgement of their uncertainty.

The speed and versatility of the streamline technology have led to many other new applications such as the following: 
- Swept Volume Calculations

- Rate Allocation and Pattern Balancing

- Modelling Tracer Flow and Waterflooding

- Ranking Geostatistical Models

- Upgridding and Upscaling

- History Matching/Production Data Integration

- Primary Recovery and Compressible Flow

- Solvent Flooding and Compositional Simulation

Streamline methods are particularly well suited for modelling and interpretation of tracer tests because of their ability to control numerical dispersion. Streamline methods have also been used successfully for modelling waterflooding both in fractured (dual porosity and dual permeability) and unfractured reservoirs. Speed-up factors of 1-1000 over the conventional finite-difference simulators have been reported in the literature, although this depends on the application [23].

\subsection{Objectives of the Thesis}

The objectives of the research project areas are as follows:

- To quantify and attempt to eliminate the differences between finite difference simulation and streamline simulation for gas displacement processes.

- To develop some guidelines on the use of streamline simulation for gas displacement processes.

The concept of streamline technology in order to achieve the objectives above can be explained this way in very simple terms:

Streamlines represent reservoir movement/dynamic response to the velocity field (in this application, this is the total multiphase Darcy velocity divided by the porosity) through strict observation of differences in area pressure/spatial representation. This concept is simply looked at in form of "Time of Flight". Time of flight is the time taken for a flux line or a pressure response to move from one point to another. In this 
way, fluid leaving an injector for example can be tracked. Thus, how fast fluid moves can be monitored particularly in regions of higher permeability, the tracer moves faster, leading to early breakthrough.

\subsection{Thesis outline}

The outline of the thesis is as follows:

Chapter 2 focuses on a discussion and introduction of both Finite Difference Simulation and the streamline and its uses in the oil and gas industry as it has been confirmed and implemented successfully in the area of the water flood management (incompressible fluids).

In Chapter 3, a detailed discussion on the methodology and mathematical modelling related to both Finite Difference and Streamline simulation and governing equations.

Chapter 4 introduces and describes the previous work that has done using the Tenth SPE Comparative solution project using water injection in both fine and coarse grids. In this thesis, this model will be converted into other different recovery schemes in order to test the gas displacement process.

Chapter 5 concentrates on the simulation modelling aspects and conversion of the bases case of the Tenth SPE Model. Three cases were presented for the $10^{\text {th }}$ SPE model. One was for Water Injection, which was then converted in to a Water Alternate Gas Scheme, and the third one was the base case Gas Injection model.

In Chapter 6, the base line gas injection case is presented after using the live oil relative permeability data and the SL simulation model is run fully to the end of the simulation period. Tuning options for the saturation solver were used in order to improve the SL simulation model performance and minimise the relative error between FD and SL simulation. Additional runs to assess the impact of different injection rates and bottom hole pressures as well for full assessment. The results indicated that SL model produce similar results compared to FD with significant reductions of TCPU used.

In Chapter 7, highlights the added value of introducing the high-resolution grid of the same base case gas injection model. This has been done by constructing a new high-resolution grid that captures and maintained all the heterogeneities in the bases case model in both Porosity and Permeability as we 
highlighted the benefit from high resolution grid ran by SL with significantly less CPU. Results indicated that SL is efficient and produce comparable results with FD model.

In Chapter 8, the baseline gas injection case is modified to compositional mode and miscible flood cases using different sets of PVT and relative permeability data. The results showed an overall good match in all parameters. The same tuning parameters were used in the compositional mode and the overall relative error noticed is low and within the acceptable limits.

Finally, overall conclusions and the future work are discussed in Chapter 9. 


\section{Chapter 2}

\section{Literature Review}

\subsection{Introduction to Computational Fluid Dynamics (CFD)}

The high-speed digital computer developments in the last century had a remarkable impact on the way principles of fluid mechanics and heat transfer were applied to modern engineering practice and design. Computational methods were made available that before would have taken years to work out and solve a problem, and can now be solved at very little cost in a few seconds of computer time. This does not mean that computers run today only in seconds. On the other hand, many computational tasks today take days of CPU time, as the complexity of problems that can be tackled have increased significantly, and we still even need more powerful computers to accurately simulate the flow inside porous media [12].

The history of CFD is tied to the development of the modern digital computer and most of the problems were solved using either analytical or empirical methods until 1955. Before this time, a few pioneers used numerical methods to solve problems as the calculations used to be performed by hand and only a single solution was presented. Then, the digital computer was developed and the actual start of CFD occurred. During the Second World War, a huge amount of research was done using numerical methods for solving problems of fluid flow. During this time, Professor John von Neumann developed a new method to evaluate the stability of numerical methods for solving time-marching problems. Later on in 1950, a detailed description of the von Neumann method and this work was very significant as it presented a practical way of evaluating the stability. This method is one of the most common methods used in CFD [13][14].

Finite difference methods generally are serving the point of departure for CFD analysis. The principal idea of CFD methods is to replace a governing partial differential equation by an equivalent set of algebraic equations and it is one of several options for this discretisation of the governing equations. The basis of finite difference methods is the Taylor series of expansion of a function that considers that a continuous function $f(x)$ and its value at neighbouring points can be expressed in terms of a Taylor series as follows: 
(1) $\mathrm{f}(\mathrm{x}+\Delta \mathrm{x})=\mathrm{f}(\mathrm{x})+\mathrm{df} / \mathrm{dx}(\Delta \mathrm{x})+\mathrm{d}^{2} \mathrm{f} / \mathrm{dx}^{2}\left(\Delta \mathrm{x}^{2}\right) / 2 !+. .+\mathrm{d}^{\mathrm{n}} \mathrm{f} / \mathrm{dx}^{\mathrm{n}}\left(\Delta \mathrm{x}^{\mathrm{n}}\right) / \mathrm{n} !+.$.

The term $\mathrm{df} / \mathrm{dx}$ in this series expansion can be rearranged to give:

$$
\mathrm{df} / \mathrm{dx}=[\mathrm{f}(\mathrm{x}+\Delta \mathrm{x})-\mathrm{f}(\mathrm{x})] / \Delta \mathrm{x}-\mathrm{d}^{2} \mathrm{f} / \mathrm{dx}^{2}(\Delta \mathrm{x}) / 2 !-\ldots-\mathrm{d}^{\mathrm{n}} \mathrm{f} / \mathrm{dx} \mathrm{x}^{\mathrm{n}}\left(\Delta \mathrm{x}^{\mathrm{n}-1}\right) / \mathrm{n} !-\ldots
$$

Or

$$
\mathrm{df} / \mathrm{dx} \approx[\mathrm{f}(\mathrm{x}+\Delta \mathrm{x})-\mathrm{f}(\mathrm{x})] / \Delta \mathrm{x}+\mathrm{O}(\Delta \mathrm{x})
$$

\subsection{Law of Conservation of Mass}

The Darcy equation was developed in 1856 by Henry Darcy a French Hydrologist who studied flow of water through porous media and the fundamentals of flow through porous media. This law is proportional to the pressure gradient and inversely proportional to the viscosity of the fluid and on the bases of the primary differential from the equation of single-phase flow.

$$
\frac{q}{A}=-\frac{k}{\mu} \frac{\partial P}{\partial x}
$$

Where

$q=$ volumetric flow rate, $\mathrm{ft}^{3} / \mathrm{s} \quad \mathrm{A}=$ area perpendicular to flow direction, $\mathrm{ft}^{2}$

$k=$ permeability, milli Darcy (md), ${ }^{\mu}=$ dynamic viscosity of flowing fluid, centipoise,

$P=$ pressure, psi, $\quad x=$ length, $\mathrm{ft}$

The flow equation describes the flow of fluids from a reservoir rock to its pore network and under reasonable pressure differentials. The law of conservation of mass states that over a given period of time, the sum of all the mass that flows in and out of any system, is the change in mass in that block. Figure 2.1 shows the fundamental principles of the discretisation of the equation for flow through porous media. 


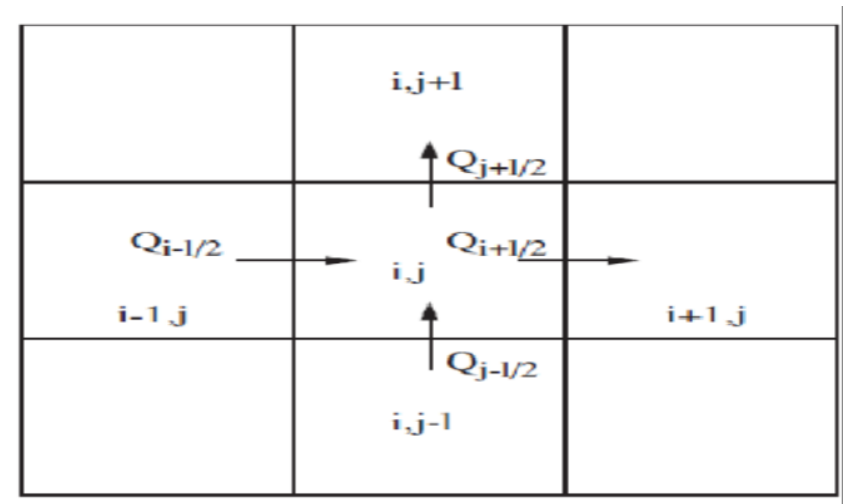

Figure 2-1: Fundamentals principles of Flow through Porous Media [12]

Principals of the flow in porous media are mainly bases on the combination of both Darcy's Law and conservation of Mass Law and mainly governed by the flow through the porous media, which derived from the combination of Darcy's Law and mass balance equation.

\subsection{Single phase 1D Pressure Equation}

In fluid mechanics a one-dimensional flow is defined as a flow in which all flow is parallel to a plane and the properties of the flow do not change when the fluid is flowing perpendicular to the plane. In other words, the flow parameters such as velocity, pressure, density etc at any point in time only vary in the direction of flow and not across the section. An example of a 1D flow is laminar flow of fluid through a pipe. In this section, the single-phase one-dimensional pressure equation without gravity effects would be obtained. For the 1D flow, the control volume is a block i, and the flow area A, and depth $\mathrm{H}$, of the reservoir are assumed to be a function of the single space variable $\mathrm{x}$.

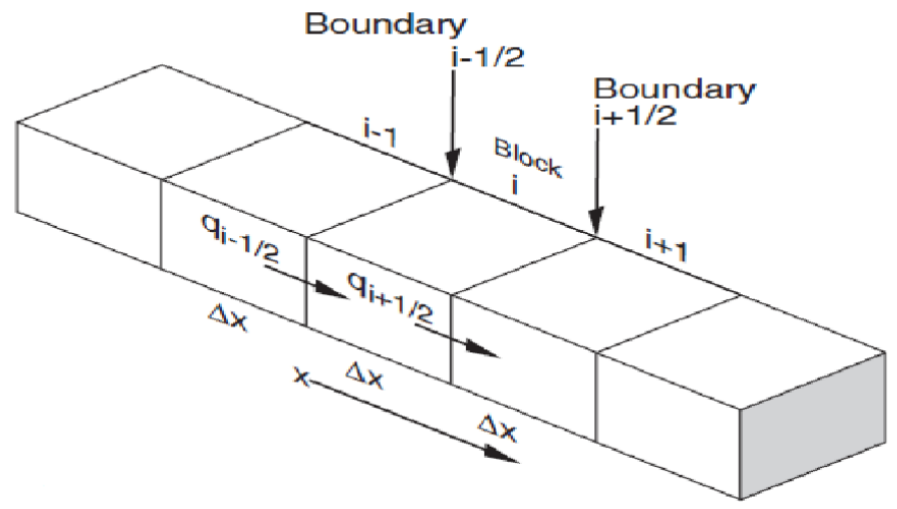

Figure 2-2: Material Application for the single-Phase Equation in a Control Volume Grid Block. [12]. 


\subsection{Single Phase Two-Dimensional (2D) Pressure Equation}

In reality flow occurs in the three coordinate direction $(\mathrm{X}, \mathrm{Y}$ and $\mathrm{Z})$, i.e, it is in three-dimensional (3D); in practical solution it is possible to assume that the flow occurs in a set of parallel planes with no flow normal to them and this flow is similar in each of these parallel planes. Concerning the 2D case, the control volume is ( $i$ and $j$ ) and the depth of the reservoir is considered to be a function of the space variable and $y$ i.e $\mathrm{H}(\mathrm{x}, \mathrm{y})$. The mass of the fluid into the grid block is equal to:

$$
\tilde{q}_{i j} \times \rho_{i j} \Delta x \Delta y \Delta z
$$

Where:

$$
\begin{aligned}
& \tilde{q}_{i j}=\text { well flow rate bbls/day } \\
& \begin{aligned}
\rho_{i j} & =\text { flowing fluid density, } i b / f t^{3} \\
\Delta x \Delta y \Delta z & =\text { Volume of block }(i, j), f t^{3}
\end{aligned}
\end{aligned}
$$

Figure 2.3 shows the control volume block $(i, j)$ for a 2D system.

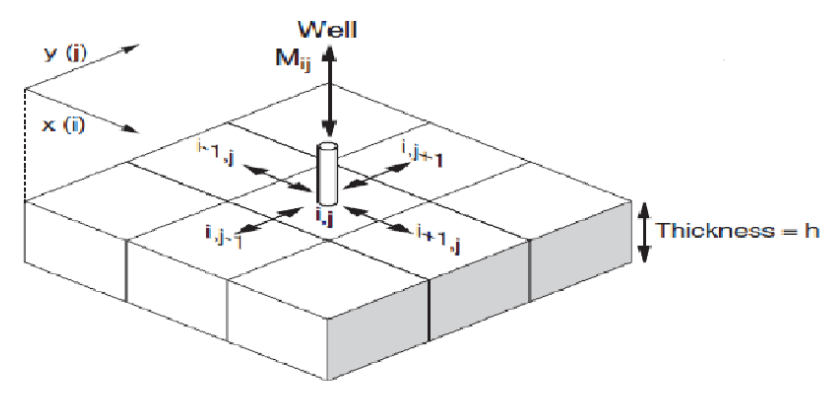

Figure 2-3: The control volume of 2D $x, y$ grid, Block $(i, j)[12]$.

\subsubsection{Explicit vs. Implicit Methods}

Explicit method are simple to program and allow marching forward in time point by point from given initial condition and it is generally less stable than implicit methods and may give an unphysical solution if the marching time step is too large. However, implicit methods are more difficult to program and require simultaneous solutions of algebraic equations at each time step to get the solution [16][17]. 
As per Runge-Kutta methods, an example of an explicit method is Euler's method which is expressed by the following equation:

$$
y_{1}=y_{0}+h f\left(x_{0}, y_{0}\right)
$$

However, the simplest example of an implicit method is the backward Euler method, as described in the following equation:

$$
y_{1}=y_{0}+h f\left(x_{1}, y_{1}\right)
$$

Where: $\left(x_{0}, y_{0}\right)$ is the initial point required to initiate the new point $(x 1, y 1)$, where $x_{1}=x_{0}+h \mathrm{x} 1=\mathrm{xo}+\mathrm{h}$ and $h \mathrm{~h}$ is a so-called "step size"[77].

\subsection{Streamline simulation}

A simple definition of a streamline is a path following the instantaneous fluid velocity within a reservoir system, and it defines these flow paths then models the fluid displacement along them by generating numerical solutions to the governing fluid equations in one dimension [45].

This technique decouples the computation of saturation and the pressure variation in time and space. The pressure field is initially solved by the finite difference method using a specific time step, which is independent from that used in the saturation solution. The velocity field is then computed from the pressure field and the streamlines are traced according to the method of Pollock (1988) [44]. The new saturation field is then updated several times using a smaller time step than that originally used for the pressure field.

The introduction of a hybrid streamline was introduced in 1981 to model large-scale chemical floods [1]. Their approach was that finite difference simulators with fully detailed physical descriptions could not be used for large-scale simulations, while stream tube-models that account for large-scale simulators were seriously deficient in displacement physics. They proposed to solve detailed cross-sectional simulations using differences and then map these pseudo-solutions along areal streamlines as a way to predict field performance of the chemical flood [1][47]. 
Today's modern engineering workflows require many forward simulations and the computational load using finite differences to accommodate such workflow, particularly with the use of advanced displacement physics and geostatistics-driven reservoir models, is simply too high for field performance predictions and management. Because of the efficiency of this method, large time step sizes are used and engineers can simulate 100+ well models with 500,000+ active cells in tens of minutes rather than days. It is suitable for injection/production type displacements (water, miscible gas) or even strong aquifer systems [9].

Modern day streamline based flow simulation differentiates itself from cell based simulation techniques such as finite differences and finite elements in that components are transported along streamlines rather than moved from cell to cell. This difference enables streamlines to be extremely efficient in solving large field scale, and geologically complex situations. The basic element of streamline simulation is its dual-grid approach. The traditional static (Eulerian) grid is used to specify petro-physical properties, well locations, rates and initial conditions, and to solve for the spatial pressure distributions using an IMPES formulation when we use Implicit pressure and Explicit Saturation (IMPES). [50] [56] [57].

The dynamic (Lagrangian) grid represented by the streamlines is used to solve hyperbolic equations that determine the transport of chemical species. The speed of novel data and streamline simulation, such as well factors and well drainage/irrigation zones, have made streamlines an important, complementary approach to traditional simulation approaches in reservoir and production engineering workflows such as sensitivity runs, evaluating up-scaling algorithms, flow management and history matching. The distinguishing feature of modern streamline simulation to the approach of the late 1970s and 1980s is the $3 \mathrm{D}$ nature of the streamline versus $2 \mathrm{D}$ and the ability to update the streamlines as well change in conditions and the inclusion of gravity [50].

\subsubsection{Stream tubes and Streamlines}

Both streamline and streamtube methods have been used earlier for many years for computational fluid flows, and it was reported for the first time in the 1950s. During the last decades, it has solved the complex fluid flow models for multiphase flow in porous media with a reasonable balance between the 
physics modelled and computational efficiencies. As the oil and gas reservoir models have grown in complexity and its geological models have several million grid cells, the computers have become more powerful toward developing reservoir simulators based on finite difference (FD). However, the conventional FD methods suffer from numerical smearing and inefficiencies for multimillion grid cell models [18]. Streamline-based flow simulation has made significant advancement in the last decades. Streamline simulation is based on a sequential approach; the pressure solution is calculated at the end of the time step based on the saturations at the beginning of the time step. The boundary conditions are generally based on open wells with given rate targets and limits, aquifer modelling, pressure boundaries and flux boundaries defined by the user. Streamline simulation is an IMPES type reservoir simulation, solving pressure implicitly, then the saturation explicitly. It operates on the principal of decoupling the pressure equation from the saturation, which helps in decomposing a heterogeneous 3D into a number of 1D streamline where all fluid computations are completed. A generalised flow diagram of streamline simulation is presented in Figure 2-4. As per this flow diagram, grid geometry, rock and fluid properties, well locations, production/injection data and boundary conditions are the main input date required. Then, the 3D grid will be initialised, and using finite difference approximations, the pressure distribution is derived to generate velocity vectors which are perpendicular to the predicted pressure contours. This velocity field is used to trace the streamlines. Wells (source or sink) plus the boundary conditions govern the initiation and ending of all traced streamlines, and make a second grid system to help and solve the fluid flow equation along the streamline.

The second grid system works with a local time step controlled by fluid migration calculations. The solution obtained from this grid is then mapped into the 3D Cartesian grid to honour the saturation distribution. Pressure is solved again and the streamlines are remapped. This process will be repeated until the end of the simulation, and the dual grid approach is the underlying difference between streamline simulators and finite difference methods [55][58]. 

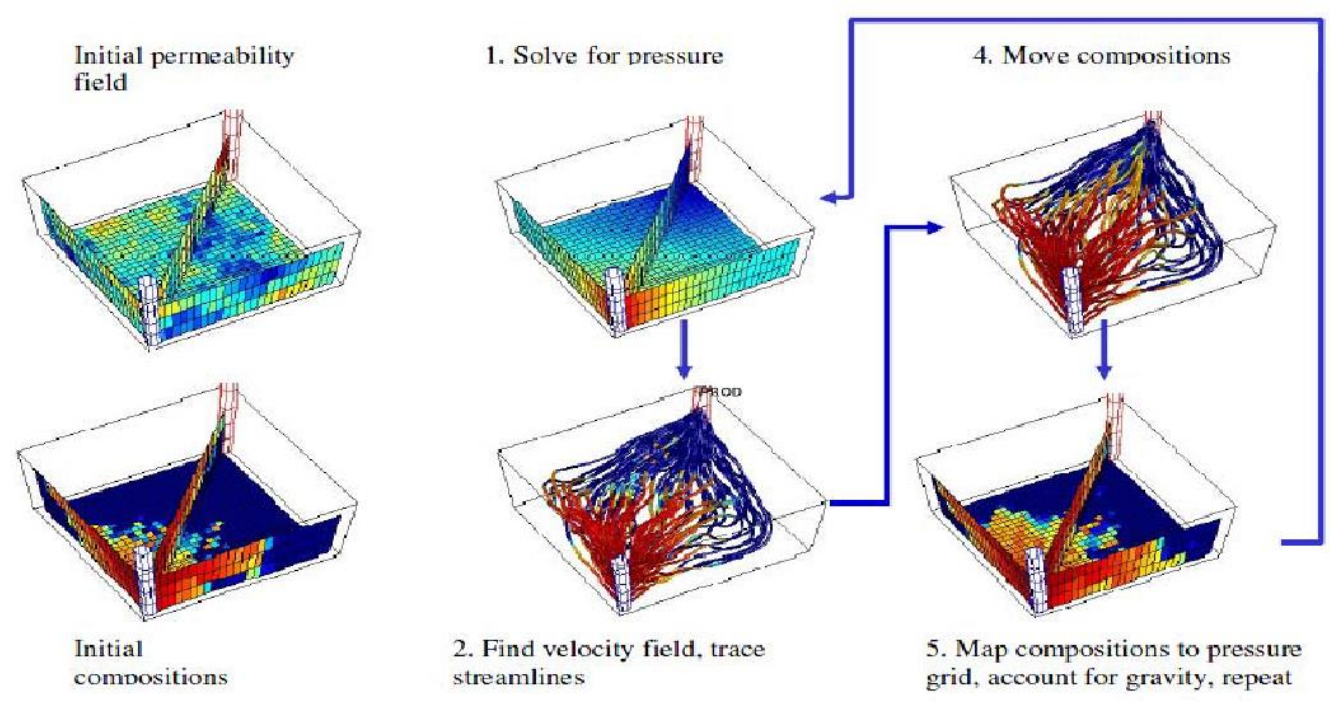

Figure 2-4: Streamline Simulation Flow Diagram [64].

There are many advantages of implementing streamline simulation for reservoir modelling. One of these advantages is the computational speed, which attracted reservoir engineers initially to this technology. This is mainly due to the fact that 1D transport calculations are not constrained by grid size and permit the use of larger time steps. Streamline simulation has been reported to be 1 to 3 times faster than the conventional finite difference simulation [56].

Streamtubes and streamlines are well known in Computational Fluid Dynamics (CFD). In the petroleum industry, numerical computation of streamtubes has been applied to two-phase and three-phase flow in homogeneous, areal systems for several well patterns with the assumption that the geometries of the streamtubes does not change throughout the displacement process. Under this assumption, the Buckley-Leverett theory was used to calculate the fluid displacement along the streamtubes. Although the geometries of streamtubes were fixed throughout the displacement in their work, good agreement with experimental results were obtained [16].

A new method has been developed to update the streamtubes method, and this was introduced in order to honour the changing mobility field. The result of this work was compared with those obtained by Higgins et al [17] and it showed that the fixed streamtubes approach is satisfactory for most two-phase problems for mobility ratios ranging from 1 to 1000 [17]. 
Streamline simulation has also been used in detailed history matching processes, as about $80 \%$ of the history matching of the preferred geological realisations has been performed using Streamline simulations. When comparing these history matched models done by streamline simulation with the finite - difference simulators before moving into predictions, the results showed an excellent agreement as shown in Figure 2-5 [76].

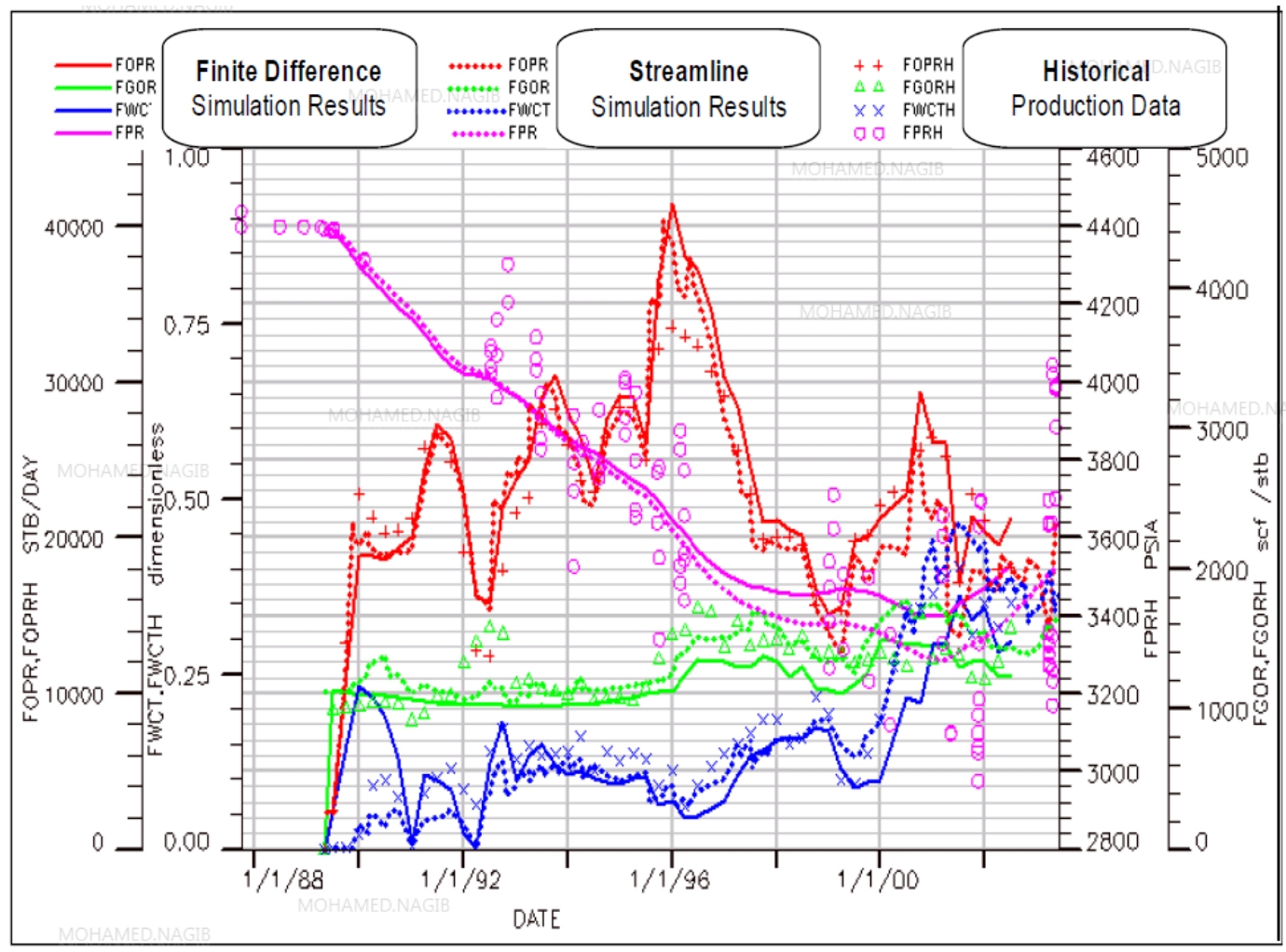

Figure 2-5: Comparison of the Full Field History Match Results with the Streamline and Finite Difference Simulators [76].

Streamline Simulations have now been established as a reservoir engineering tool that is well-suited for geologically complex, heterogeneous reservoir systems and for convection dominated flows. In many applications, streamline simulation offers particular advantages over conventional finite-difference simulation, for example for fast simulation of waterflood and assisted history matching of mature fields. Streamline models also have the added feature of flow visualisation, which makes it physically intuitive. Its speed and accuracy can be used for identifying swept and un-swept regions in waterflood, i.e. flood fronts, establishing injector-producer relationship, tracer transport, water-flood allocation, predicting water breakthrough optimising water injection, management of waterflood, identifying reservoir 
compartmentalisation and statistical ranking of stochastic geo-models, using the concept of effective density [18]. The lack of understanding of numerical stability and error estimates has posed a problem on the choice of the time-stepping in the streamline simulators. However, a novel approach has been presented for the selection of time steps during streamline simulation, which is based on three factors. Firstly, the reformulation of the streamline equation is used to capture 3-dimensional flux terms. Secondly, a grid-based corrector algorithm is formulated to update the saturation to account for the transverse flux. Thirdly, a discrete CFL (Courant-Fredrich-Levy) technique was applied to ensure the stability and provide effective time step control [19].

\subsection{Streamlines in 3D using the Time of Flight Technique}

One main feature of the current SL simulation is that the streamlines are truly $3 \mathrm{D}$ rather than $2 \mathrm{D}$ as in the streamline and streamline methods of the 1970s and 1980s. While 2D streamlines are generally depicted from a bird's eye perspective, 3D streamlines now correctly account for the previously missing third (vertical) flow component. Adding the third dimension has been critical to current usage of the technology, thus capturing the cross-flow between layers and the flow around geological barriers, and more importantly gravitational effects. From a practical point of view, the availability of 3D streamlines no longer requires geological models to be transformed into pseudo 2D areal homogenous models. The models retain a full 3D, geocellular description, and the streamlines can appear to be crossing in a 2D plan view - as in Figure 2-6 and this is because of the added dimensionality of the problem. 


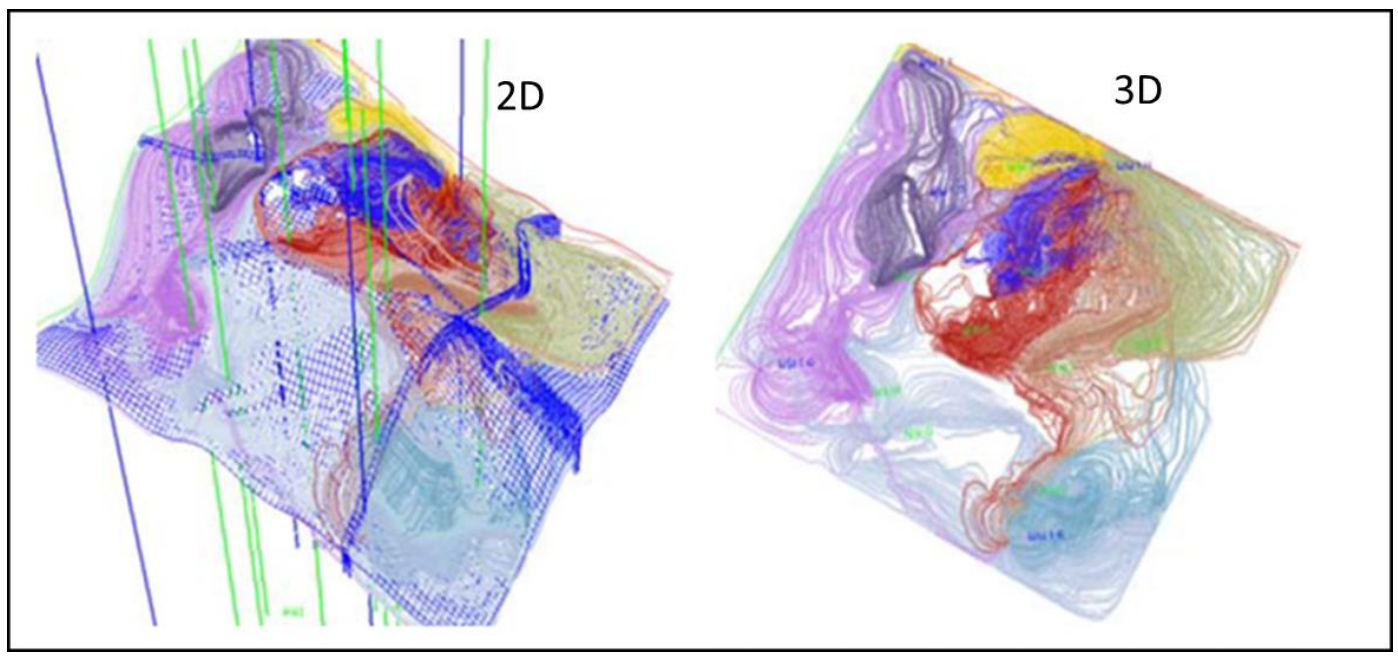

Figure 2-6: 2D (right) and 3D (left) that Properly Accounts for the Vertical flow Component [23].

Breakthrough work was done by Pollock in 1998 for the efficient tracing of streamlines in 3D. His method is simple and it is formulated in terms of a time of flight (TOF) coordinate [54]. When applying Pollock's tracing method to any of the cells, the total flux in and out of each of the cell boundaries is calculated using Darcy's law. When the flux is known, the algorithm centres on determining the point of exit of the streamline and the time to exit given any entry point assuming a piece-wise linear approximation for the velocity field in each coordinate direction. The assumptions inherent in the 7-point stencil used in finite-differencing are used, namely if $v$ is the interstitial velocity $(\nu=\mu / \phi)$, then a linear, independent velocity description in the $\mathrm{x}$-direction is assumed, and is given by:

$$
v_{x}=v_{x 0}+g_{x}\left(x-x_{0}\right)
$$

Where $V_{\mathrm{x} 0}$ is the $\mathrm{x}$-velocity at $\mathrm{x}=\mathrm{x}_{0}$ and $g_{\mathrm{x}}$ is the velocity gradient in the $\mathrm{x}$-direction, estimated using finite differences:

$$
g_{x=} \frac{v_{x+\Delta x}-v_{x 0}}{\Delta x}
$$

This is the linear velocity model, and it is shown in in Figure 2-7. 


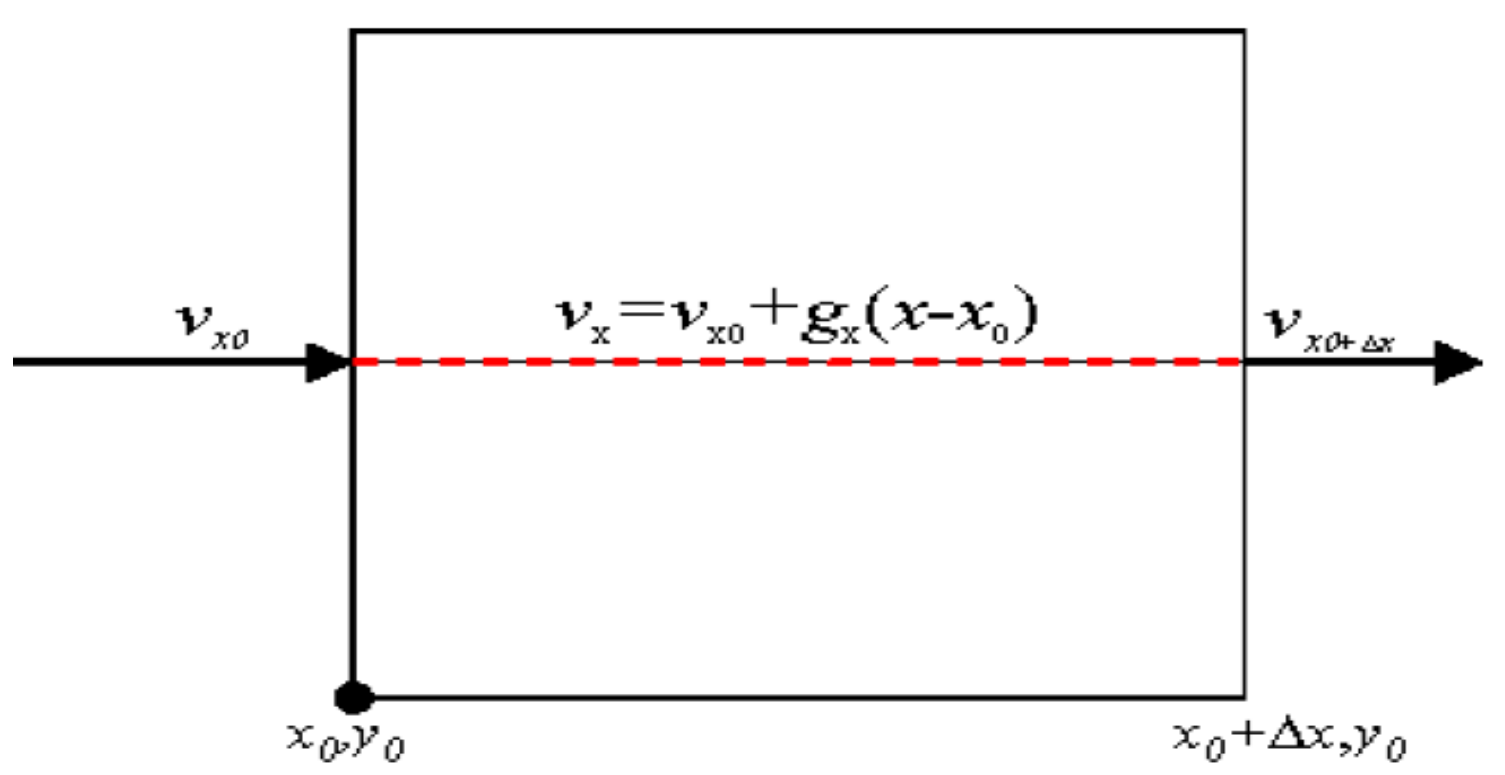

Figure 2-7: The linear Velocity Description in each Coordinate Direction [54].

Since $v_{x}=d x / d t$, equation 2.8 may be integrated in each coordinate direction to determine the position vector as a function of time within the cell as follows:

$$
\begin{aligned}
& x_{e}=\frac{1}{g_{x}}\left(v_{x i} \exp \left(g_{x} \Delta t_{m}\right)-v_{x 0}\right)+x_{0} \\
& y_{e}=\frac{1}{g_{x}}\left(v_{y i} \exp \left(g_{y} \Delta t_{m}\right)-v_{y 0}\right)+y_{0} \\
& z_{e}=\frac{1}{g_{x}}\left(v_{z i} \exp \left(g_{z} \Delta t_{m}\right)-v_{z 0}\right)+z_{0}
\end{aligned}
$$

Equations (2.9) are then inverted to estimate the time of flight in each coordinate direction, as given in equations (2.9). Figure 2-8 indicates the time it takes for a particle to exit in each coordinate direction.

$$
\Delta t_{x}=\frac{1}{g_{x}} \operatorname{In}\left[\frac{v_{x 0}+g_{x}\left(x_{e}-x_{0}\right)}{v_{x 0}+g_{x}\left(x_{i}-x_{0}\right)}\right]
$$




$$
\begin{aligned}
& \Delta t_{y}=\frac{1}{g_{y}} \operatorname{In}\left[\frac{v_{y 0}+g_{y}\left(x_{e}-x_{0}\right)}{v_{y 0}+g_{y}\left(x_{i}-x_{0}\right)}\right] \\
& \Delta t_{z}=\frac{1}{g_{z}} \operatorname{In}\left[\frac{v_{z 0}+g_{z}\left(x_{e}-x_{0}\right)}{v_{z 0}+g_{z}\left(x_{i}-x_{0}\right)}\right]
\end{aligned}
$$

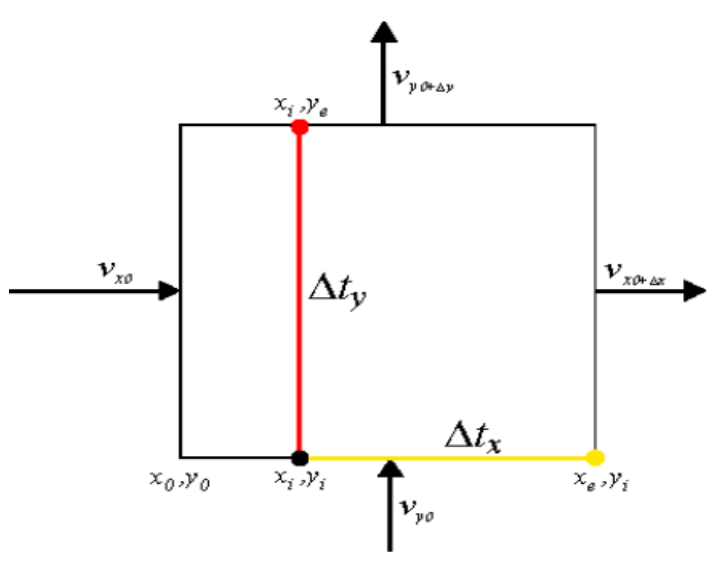

Figure 2-8: The time it takes for a particle to exit in each coordinate direction given the point [23]. Finally, we use:

$$
\Delta t_{m}=\operatorname{MIN}\left(\Delta t_{x}, \Delta t_{y}, \Delta t_{z}\right)
$$

The 3D tracing method through a Cartesian cell is shown in Figure 2-9.

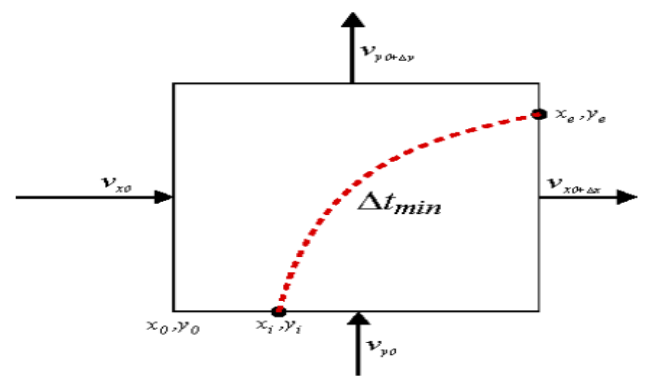

Figure 2-9: The 3D tracing method through a Cartesian Cell [54]. 
After the end point location $\left(x_{e}, y_{e}, z_{e}\right)$ is known, it will become the inlet position of the neighbouring block, then the streamline can be traced from block to block as shown Figure 2-10, and this is based on the assumptions of the case that has incompressible flow processes so the effects of compressibility are negligible. In this case, incompressible and immiscible fluids both gravity and capillary pressure are neglected.

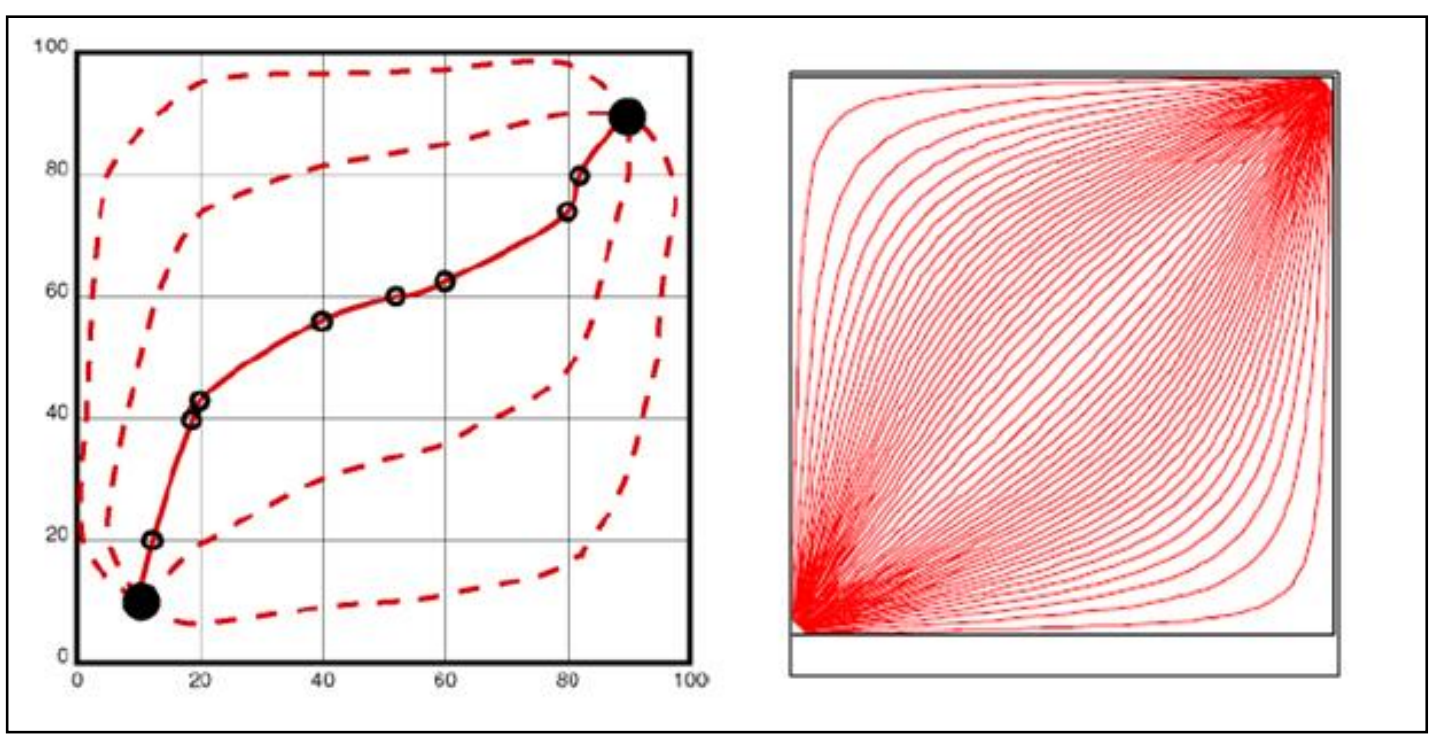

Figure 2-10: An arbitrary entry point, the time to exit and the exit point can be determined [23].

Therefore, velocity field is updated frequently and new streamlines are recomputed to reflect the nonlinear nature of fluid movement [75].

Figure 2-11 illustrates the algorithm used for 3D distribution forward in time between streamline in each time step. 


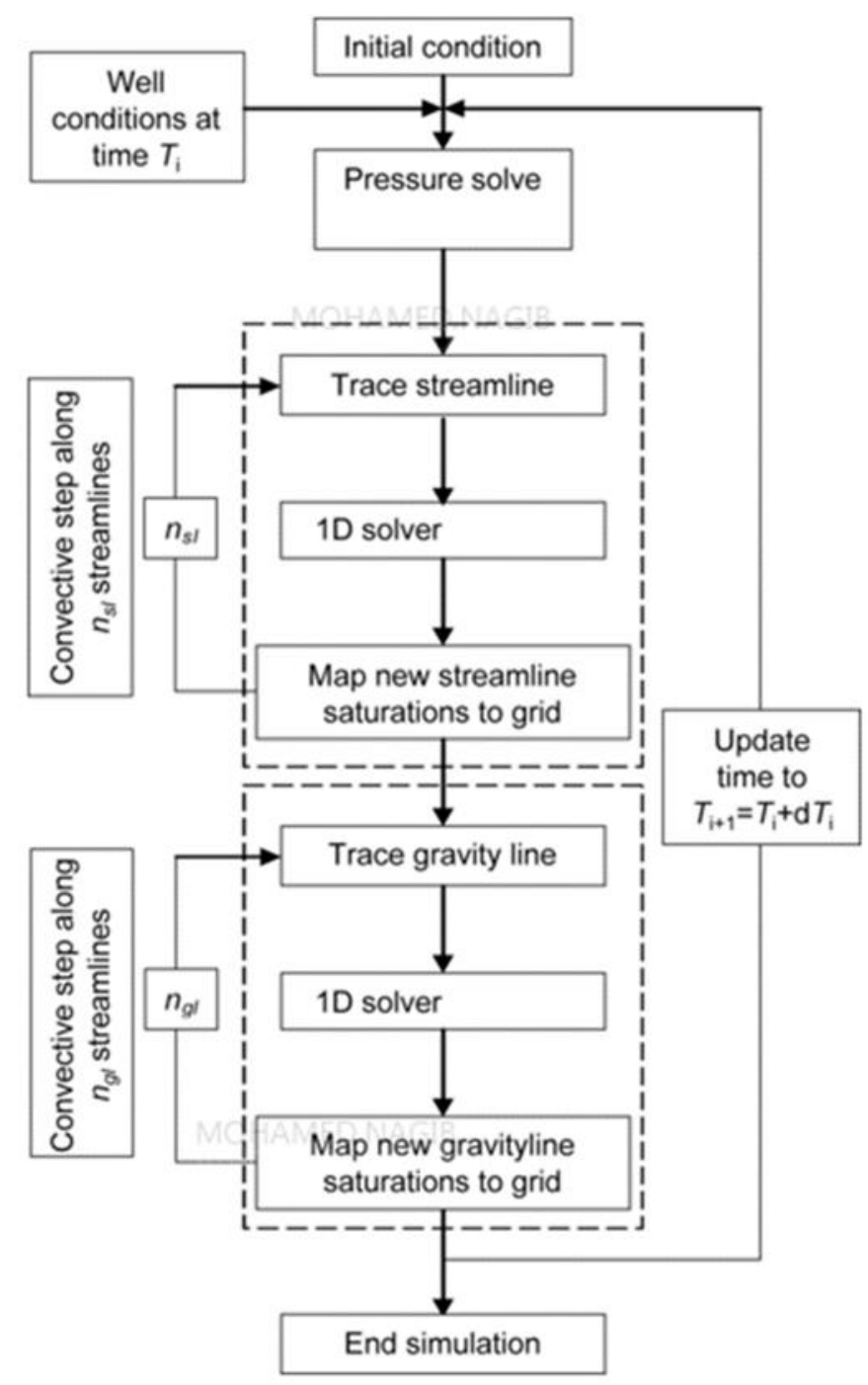

Figure 2-11: the basic algorithm for streamline simulation [75]

Streamline technology is now well known in the oil and gas industry and it has been used for many years. It has been recently noted that a large extent was driven by developments in reservoir characterisation as there has been huge progress in the ability of the industry to develop fine-scale models (model size of multi-million cells), that integrate detailed three-dimensional geologic and geophysical data. This has resulted in a steadily increasing gap between flow simulation and the static model, not only because of the size of these models, but also because of our desire to obtain reservoir performance predictions for multiple realisations of such models. Secondly, with high-resolution models comes increased acknowledgement of their uncertainty. Therefore, a demand is there in order to be able to measure the impact of the unknown elements of the static model on fluid flow and transport for sensible 
reservoir management. Recent developments in streamline simulation offer important potential to meet some of these challenges. Streamline models provide us with a tool for speedy evaluation and ranking of 3-D reservoir models [23][24][44].

\subsection{Applications of Streamline Simulation}

Many case studies have been presented on the application of streamline simulation on various areas of reservoir management. These areas include heterogeneous geologic screening, tracer modelling, water flood performance, etc. Multiple realisation is the key concept through which geological screening was achieved. Multiple models are generated and ranked to allow for better economic forecasting, particularly at early project stage. From a practical sense, multiple scenarios are framed by creating a detailed tree that is an outline for the project's workflow. Depending on the maturity of the project, different amount of data would be available, as more information becomes available; there is possible elimination of some branches of the tree as some scenarios become tightly constrained. One example of multiple realisation in the primary level may be changing the depositional environment being modelled whereas in the secondary level, we may be looking at the effect on channel orientation in a fluvial environment on field performance [24].

Streamline simulation is well known now as an ideal reservoir management tool for mature water floods as non-swept zones can be easily identified, multiple forecast scenarios evaluated, and novel information like well pair interactions obtained. Well-pair interactions are particularly useful as they allow for pattern surveillance, quantifying offset production with injection volumes, and identifying efficient versus inefficient areas of injection [25]. In this work, a 10\% gain in offset oil producers was achieved while applying pattern optimisation in water flood reservoirs. The enablement offered by pattern optimisation can lead to increasing the injection rates of some wells, diverting flow towards offset oil producers by high gross rate wells. This is demonstrated in the Thuleitat field whose complex description (multiple fluid contacts, numerous faults and stacked reservoir sections) was successfully optimised by identification of well-pairs and rebalances of well rates [25]. The benefits of streamline simulation in pattern flooding cannot be over-emphasised. It minimises runtime, allows for regional investigation of 
fluid anomalies during history match and investigates good versus bad areas of the field and reduces fluid cycling [26].

\subsubsection{Finite Difference Compositional Simulation}

In this case, the fluid model is defined by an equation of state (EOS) and its critical parameters. Critical parameters are entered for each component in order to allow the liquid and vapor phases to be split down into their components. Compositional models typically recognise and determine the lighter hydrocarbons. They then create single or lumped components for $\mathrm{C}_{7}+$ or more, pseudo components are defined by their molecular weight and specific gravity [28].

Existing compositional simulators (FD) such as E300 use multipoint spatial discretisation to account for tensor permeabilities with PVT described by EOS. Conservation component equations are then written for each component [31].

As mentioned earlier, streamline-based techniques were developed mainly to make use of the highspeed computations with the ability to take large global time steps as FD usually needs excessive numerical dispersion, and small-time steps.

\subsubsection{Streamline Compositional Simulation}

First compositional streamline simulator was developed in 1997 to study miscible/immiscible gas injection. In this simulator, a 1D compositional FD method was used to move components numerically along streamlines and then map the saturations back on the 3D grid and finally obtain a 3D compositional solution. This streamline method assumed the pressure to be constant throughout all time steps, resulting in inaccurate calculation when extended to the 3-phase mode [27]. The black oil streamline formula was extended in 2006 to became more generalized form by including compressibility effects in their threephase black-oil simulator. A new approach was proposed for history matching three-phase flow using a novel compressible streamline formulation and analytic sensitivities. In this approach, a generalised streamline model was used to calculate the compressible flow by introducing an effective density of the total fluids in order to capture the changes in fluid volumes with pressures before tracing the streamlines. Then, an accurate way was found to calculate the actual GOR based upon the sensitivity parameters that defined the relation between the reservoir properties and the production response, water cut and GOR. These sensitivities became an integral part of the history match process [32]. 
A systematic approach to include gravity in streamline-based compositional simulators was introduced in 2004. This approach was to use 1D fully implicit solutions of FD simulation, to solve for pressure and fluid compositions together along each streamline, accounts for the changes of the phase behaviour that depend on the changes in pressure. The method was tested and compared against the FD compositional simulator using the SPE 9 Model. This work on SL showed that it is significantly faster and requires less memory [29][30].

The error dependence on the number of streamlines was examined in order to provide an analytical proof and numerical demonstration of the order of spatial convergence of the mass balance discretisation error. The results showed the impact of stagnation regions of the order of convergence. However, a transverse error was due to a faulted grid at the cell faces resulting in a problem on the well trajectories. This error can only be solved by introducing additional streamlines, and this might lead to inaccurate time of flight and material balance error [31].

The cumulative volume was used instead of time of flight in 2008. This approach was implemented in a 3D two-phase streamline simulator based on some Brazilian oil field data and using the SPE $10^{\text {th }}$ comparative case. The results showed that it can be substantially faster than the FD simulation [33].

The streamline simulator was extended in 2011 to account for the two new phase behaviour algorithms by assuming thermodynamic equilibrium along streamlines. This method was applied to one of the $\mathrm{CO}_{2}$ projects in Canada and showed results similar to the results that were obtained by an FD simulator with a significant reduction in CPU time [34]. A 3D 3-phase compositional simulator was developed in 2014 for $\mathrm{CO}_{2}$ injection accounting for both gravity and capillary effects using orthogonal projection. This proposed approach enables larger time steps to be taken compared with conventional operator splitting. The results showed a good agreement and greater efficiency in comparison to the FD compositional simulator [35].

A compositional streamline simulation was developed mainly to integrate with an existing finite difference simulator. Pressure is computed using finite difference and velocity calculated using Darcy's law. The velocity field is assumed to change linearly in each direction within the grid blocks. Then, 
streamlines are traced from injectors to producers using Pollock's Method 1998, later on Time of flight was introduced as well.

For each streamline, point time of flight recorded along all the streamlines. Permeability heterogeneity, total mobility, and well locations determine the streamline paths. 3D material balance transformed to 1D equation along the streamlines. Then, Saturations, densities, and mole fraction mapped from the FD grid to the streamline points. Flash calculation performed at the streamline points to determine the phase compositions and then the fluxes evaluated [73].

A 1D solver is used and overall compositions of each component is updated by taking multiple time steps along streamlines. Figure 2-12 represents the 1D solver and Flux calculation in each time Step [73].

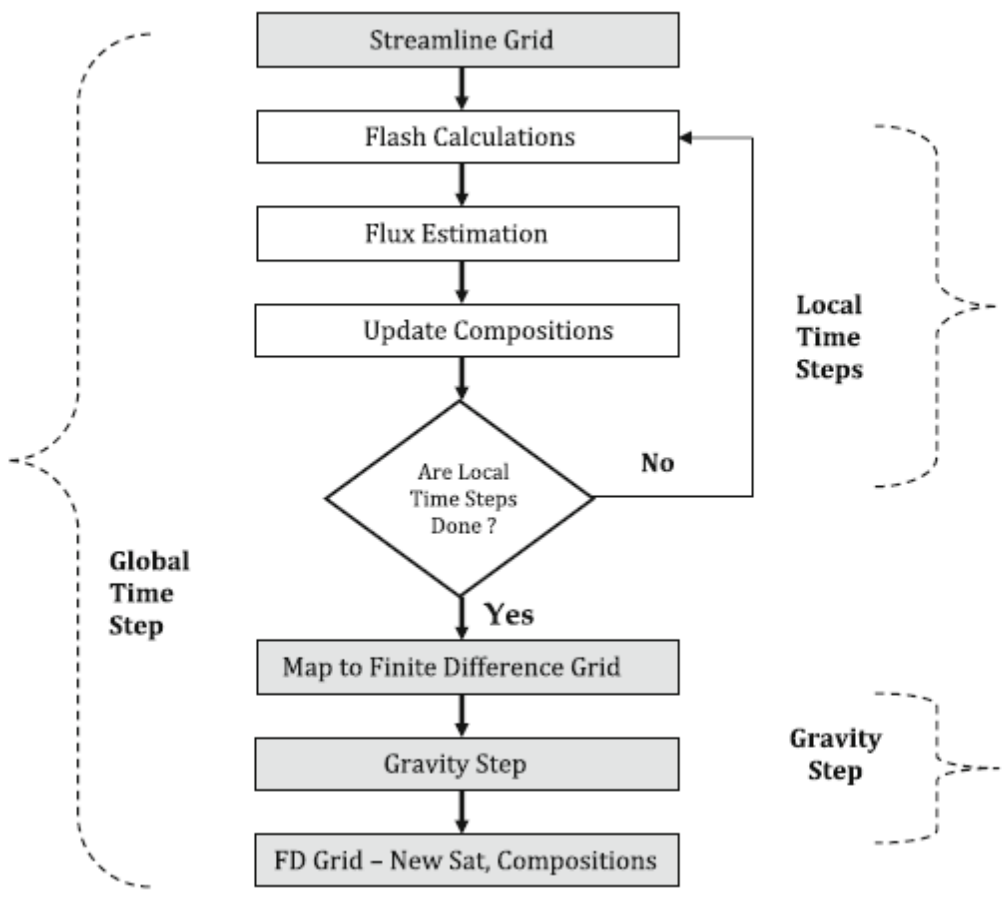

Figure 2-12: 1D solver and Flux calculation at each time step [73].

\subsubsection{Compressibility and Streamline Simulation}

As normally stream lines starts in a source (Injector) and end in a sink (Producer), all streamlines theories were developed based upon the assumptions of incompressible flow in which mainly due to the constant flow rate along each streamline. So, basically simplifying the assumptions that particularly suitable for SL simulation. The flow transport along a streamline only involved in solving the component 
for wave speeds, with each velocity as it is allowing to calculate the volume associated with a streamline simply as the product of time of flight times the flow rate along the streamline [38].

For compressible flow case, streamline can start or end in any grid blocks that acts as a volume source or sink because of the compressible nature of the model. Streamline begins and end / or end in the far field. A conversion equation then solved along each streamline to account for compressibility. Thus, volumetric flow rate (q) along streamline is no longer constant.

In this case, an extension of the main incompressible theory is required to accommodate compressible flow along the streamline. To do so, a compressible pressure equation is applied to determine the velocity field. Streamlines begin and end in the far field and volumetric flow rate $(\mathrm{q})$ along streamlines is no longer constant and time step is restricted by pressure change [38].

\subsubsection{Innovative Approach by Streamline Simulation}

The difference in streamline simulation to conventional finite difference simulation lies in the transportation of fluid. The fluid is transported over a time step $(\mathrm{t}+\Delta t)$ along streamlines rather than from cell to cell as in the conventional FD method. Because streamlines represent an image of the instantaneous velocity field, anything assumed to move with the total velocity field will follow the streamlines until the velocity field is updated to account for its changing behaviour in time.

The geometry of the streamlines and the velocity at which components travel along each individual streamline result directly from the spatial distribution of the static petro-physical properties (e.g. permeability, porosity, and relative permeability regions) in addition to the volumes produced/injected at the wells. The ability of streamlines to visualise flow is unmistakable, even to the untrained eye. To trace the streamlines at a particular time $t$, the total velocity field is required at that instant [23].

Streamline simulation is used also for covariance localisation in Ensemble Kalman Filtering for three phase history matching. The Ensemble Kalman Filter is a sequential Mont Carlo approach that works with an ensemble of reservoir models. This method uses the covariance between measurements and model parameters estimated from the ensemble, and it was used recently in the three-phase hisory matching process with the use of streamline simulation technology [62]. 
An innovative use for streamline simulation that has recently been tested is for an improvement of polymer flood managment. The technology has been extended for polymer flooding and was tested on a field scale in Romania. Streamline simulation gives an opportunity to plot cumulative oil produced as a function of polymer injected for the well patterns, and it helps in maximising the sweep and also minimising the operating costs [63].

Another approach also to develop and demonstrate a fast and robust derivative-free workflow to improve economic values of water and gas flooding optimization using rate allocation by stream-line based simulation technique. This approach provided a powerful tool for application to water flood as the work flow provide the ability to control injector and producer flow rates for improving economics values under multiple constraints [71].

The fast marching based flow simulation technique was used as a rapid and efficient approach for optimizing $\mathrm{CO}_{2}$ and Gas injection EOR in unconventional reservoirs. The Fast Marching Method is analogous to streamline simulation approach and utilises the concept of "Diffusive Time of Flight". The DTOF is a way to represent the travel time of pressure 'front' propagation and accounts for geological heterogeneity [72].

\subsubsection{Advantages of Streamline Simulation}

The benefits of streamline simulation, such as the speed and the ability to process high-resolution grids efficiently, have been emphasised in many articles. However, knowing the limitations is also important in the application of the software.

The major advantage of streamline simulation compared with other simulation methods is the comprehensive information provided by streamline simulation. There are two particularly useful sources of data. Firstly, streamlines can outline the drainage and irrigation volume associated with the producers and injectors respectively and have the ability to know which grid blocks are associated with which well, whether injector or producer, at any particular time. This method has been used as well level assisted history matching work flows to decide how to modify static grid properties to improve the match between simulated and historically observed volumes [23][24].

The second data source comes from summing the volumetric flow rates associated with all the streamlines connecting an injector or producer pair. Doing so enables determination of the well rate allocation factors (WAFs), i.e. the percentage of flow from one well to each offset well with which it 
communicates. Thus, streamlines offer a simple solution to the challenging problem of trying to associate produced and injected volumes. Well-allocation data are critical to workflows that are based on pattern analysis and are critical to manage floods effectively.

Streamline simulation can be significantly faster than finite difference methods. The immediate application of the efficiency of streamline simulation is in the simulation of fine grids with a high level of geological detail. In addition, streamlines lend themselves to easy parallelisation.

For instance, a 1.5 million active cell water flood model of the forties field (UK) with 200+ wells and 40+ years of history, ran in less than 2.5 hours on a 2-CPU quad-core system compared to approximately 6 hours for a single-core run [53]. However, the improved computational speed and memory efficiency apply to problems that are particularly tailored to streamline simulation, such as in the case of slightly compressible systems in which the principal flow physics is the displacement of resident oil by an injected fluid. Usually, water and miscible gas or both are in the presence of strongly correlated geological features. These problems are referred to as being convection dominated (i.e. principally governed by pressure gradients rather than absolute pressure).

These cases are traditionally difficult to model with FD methods, and the use of streamline simulation can be an effective complementary approach for a broader Reservoir Engineering analysis $[25][53]$.

\subsubsection{Disadvantages of Streamline Simulation}

As mentioned earlier, knowing the limitations of streamline simulation will assist in applying the right method for a particular task. Since streamline simulation is best suited for problems dominated by convection than problems dominated by diffusive-flow physics, such as gas expansion and capillary pressure, these latter problems are more challenging for streamline simulation. The reason lies in the definition of well flow direction, an exact opposite of a streamline. However, such problems are treated effectively and efficiently in finite difference methods. Initial depletion of a reservoir creating a gas cap, followed by re-pressurisation by water injection, is a classic hybrid case. With experience, such problems 
can be solved sequentially, by the use of the FD method to solve the gas expansion, then solving the repressurisation phase with streamline simulation.

Major drawbacks of streamline simulation may come from its two core architectural features, the dual grid and the assumption that streamlines are independent of each other. The dual grid requires repeated mapping of the solution variables, pressure and overall compositions between the static Eulerian and the dynamic Lagrangian grid, which leads to a method that is inherently not mass conservative. Additionally, the independence between streamlines does not favour capturing physics that is transverse to the main direction of flow, such might be the case with gravity (driven by density gradients), transverse capillary pressure (driven by saturation gradients), diffusion (driven by concentration gradients), compressibility (in all directions), and transverse-thermal (temperature gradients) effects. These difficulties can be alleviated with an operator-splitting approach, which solves the convective part along the streamlines and the diffusive part on the Eulerian grid. In fact, modern streamline simulators can be considered a sequential multi-grid method to solve nonlinear partial differential equations, with the special feature that one grid is dynamic and streamline-based [23][24][25][54].

\subsubsection{Conclusions}

In this chapter, a discussion and introduction of the streamline technology and its uses in the oil and gas industry has been confirmed and implemented successfully in the area of water flood management (incompressible fluids).

Some trials have been performed and tested in very limited conditions for compressible fluids. It has been confirmed also that SL is faster than FD and also has an ability to handle fine grid models without upscaling, and this was confirmed for water flooding cases and will be tested for the gas displacement techniques in this work.

Incompressible flow transport along a streamline usually involved in solving for the component of the wave speeds, with each velocity as it is allowing to calculate the volume associated with a streamline simply as the product of time of flight times the flow rate along the streamline.

For compressible flow case, streamline can start or end in any grid blocks that acts as a volume source or sink because of the compressible nature of the model. Streamline begins and end / or end in the far field. A conversion equation then solved along each streamline to account for compressibility. Thus, volumetric flow rate $(\mathrm{q})$ along streamline is no longer constant. 


\section{Chapter 3}

\section{Methodology and Mathematical Model}

\subsection{Differential Equations}

The one dimensional, two phase Buckley-Leverett displacement will be solved using the finite difference solution. The Buckley-Leverett solution will be used to evaluate the approximation errors of the finite difference method [12][14][15].

$$
\begin{aligned}
& \frac{\partial S_{j}}{\partial t}=-\frac{u_{I}}{\phi} \frac{\partial f_{j}}{\partial x}, \quad x>0, \quad t>0, \quad j=w, o \\
& S_{j}(x, 0)=\text { I.C. } \\
& f_{j}(0, t)=\text { B.C. }
\end{aligned}
$$

Where $S_{j}$ represents the saturation variable and $f_{i}$ represents the conserved fluxes.

$$
\begin{aligned}
& S=\frac{S_{w}-S_{w r}}{1-S_{o r}-S_{w r}} \\
& x_{D}=\frac{x}{L} \\
& t_{D}=\frac{u_{T}}{\left(1-S_{o r}-S_{w r}\right) \phi L} t
\end{aligned}
$$

Where,

$L$ is the distance parallel to flow direction and $u_{t}$ is the fluid velocity

In terms of the non-dimensionalised variables, the Buckley-Leverett equation may be expressed as:

$$
\frac{\partial S}{\partial t}=-\frac{\partial f}{\partial x}
$$

The equation could be expanded as: 


$$
\frac{\partial S}{\partial t}=-\frac{d f}{d S} \frac{\partial S}{\partial x}
$$

but the finite difference approximation to this latter equation will not be conservative. The flux leaving one grid block must equal the flux entering the adjacent grid block [33][34].

\subsubsection{Finite Difference Approximations}

The time derivative is approximated with the following finite difference expressions:

$$
\frac{\partial S}{\partial t} \approx \frac{S^{n+1}-S^{n}}{\Delta t}=\frac{\Delta_{t} S}{\Delta t}
$$

Several different approximations can be used for the spatial derivative. Figure 3-1 illustrates the "block centred" grid that is commonly used in reservoir simulation. The dependent variable is defined at the centre of the grid block and the fluxes are evaluated across the faces separating the grid blocks.

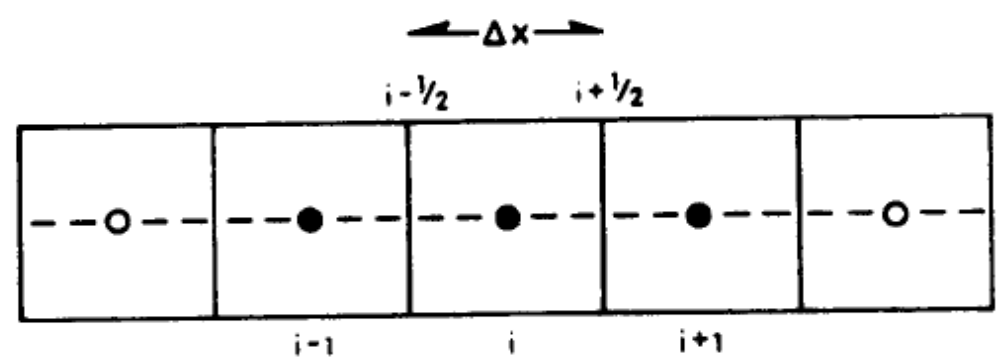

Figure 3-1: Block centred grid [15].

The divergence of the flux in grid block expressed in finite difference is the net flux from the grid block. The same concepts are applied for unequal grid spacing, but the equations have some detail not needed here.

$$
\left(\frac{\partial f}{\partial x}\right)_{i} \approx \frac{f_{i+1 / 2}-f_{i-1 / 2}}{\Delta x}
$$




\section{Central difference:}

When $f$ is a function of $\mathrm{S}$ which is evaluated at $\mathrm{x}_{\mathrm{i}-1}, \mathrm{x}_{\mathrm{i}}, \ldots .$. , the central difference approximation approximates the value at $i+1 / 2$ and $i-1 / 2$ with an average of the value on either side.

$$
\begin{aligned}
\left(\frac{\partial f}{\partial x}\right)_{i} & \approx \frac{\frac{1}{2}\left(f_{i+1}+f_{i}\right)-\frac{1}{2}\left(f_{i}+f_{i-1}\right)}{\Delta x} \\
& =\frac{f_{i+1}-f_{i-1}}{2 \Delta x}
\end{aligned}
$$

Even though the central difference approximation is a second order correct method, it suffers from problems such as severe overshoots and undershoots at discontinuities [15].

\subsubsection{Explicit or Implicit Formulation}

The finite difference approximation accuracy is dependent on the time level at which the flux terms in the equation is estimated. Figure 2-2 shows the three common time levels for estimating the flux terms. 

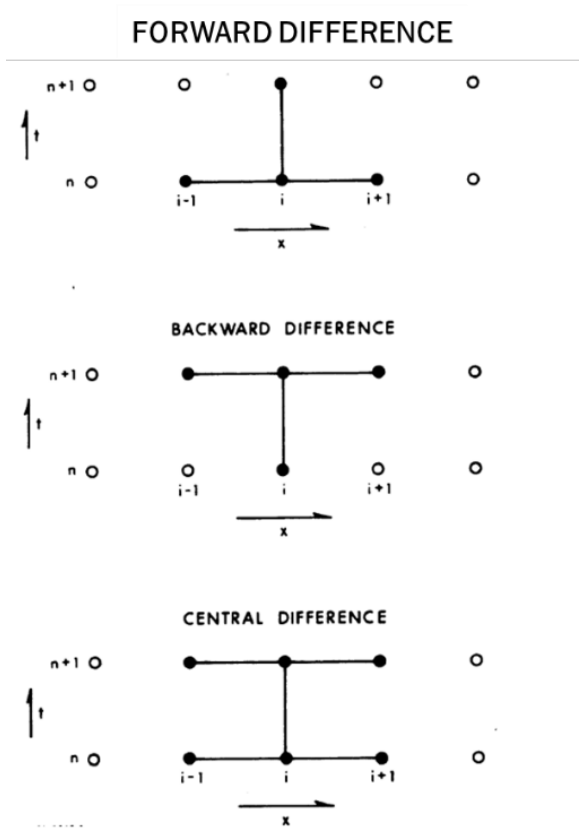

Figure 3-2: Time difference schemes [15].

The index $\mathrm{i}$ is the point in the space for this one-dimensional illustration, and it was assumed that the spatial difference terms in the flux require the values at $x_{i-1}$ and $x_{i}$, and $x_{i+1}$ for the finite difference equation. The points in space and time at which values of the dependent variable appear in the finite equations are shown in Figure 2-2 above [16][17].

\subsection{ECLIPSE Reservoir Simulation Software}

The Eclipse simulation software is a reservoir engineering software package, which handles all kinds of reservoir engineering applications, such as coal bed methane, gas field operations, calorific value-based controls, reservoir coupling, and surface networks, injection purpose, etc.

Eclipse provides solutions to the entire spectrum of reservoir simulation. It is also known as a finite difference simulator with various types of simulators such as Eclipse Black oil, Eclipse Compositional and Eclipse Thermal and Eclipse Frontsim for streamline simulation.

Eclipse has an extensive range of features to optimise finite difference reservoir simulations and to provide versatility and increased accuracy.

For the purpose of this thesis, initially will use Black oil then will upgrade the models and run compositional modelling. 


\subsection{Development of Streamline Simulator (FrontSim)}

The ECLIPSE ${ }^{\mathrm{TM}}$ simulator suite consists of three separate simulators: ECLIPSE 100 specialising in black oil modelling, ECLIPSE 300 specialising in compositional modelling and ECLIPSE FrontSim, specialising in streamline and front tracking. ECLIPSE 100 is a fully implicit, three phase and three dimensional, general purpose black oil simulator with gas condensate options. ECLIPSE 300 is a compositional simulator with cubic equation of state, pressure dependent permeability value and black oil fluid treatments. ECLIPSE 300 can be run in fully implicit, IMPES adaptive implicit (AIM) modes. FrontSim is a three-dimensional, three-phase fluid flow simulator based on the streamline concept. FrontSim can perform simulations on large and complex reservoir models several orders of magnitude faster than standard finite difference simulators, and can achieve this without grid orientation effects or numerical dispersion.

\subsubsection{Streamline Solution Strategy}

The streamline concept is based on an IMPES (Implicit Pressure Explicit Saturation) solution. The IPES method is based on a sequential approach which normally suffers from the time step length limiting CFL conditions that occurs when fluid cannot move more than one grid cell during one time step. Based upon the user defined boundary conditions within one time step the following computation is required:

- Pressure is solved with an implicit numerical method, then total Darcy velocities calculated based on the pressure potentials.

- Streamline computation to represent the computation domain for the saturation solver.

- Saturation mapping onto streamlines by solving the saturation equation individually on each streamline then solve the gravity segregation.

- Repeat the above steps in each time steps in simulation

Wells with target rates, aquifer model and pressure boundaries are the boundary conditions for each user. In summary, the pressure is used to compute a velocity field, which is used to compute streamlines. The saturation equations are solved on the streamlines using front tracking and numerical methods. The allocation factor for a producer $\mathrm{P}$, from an injector $\mathrm{I}$, is the sum of the streamline rates coming from 
injector I divided by all the streamline rates coming to producer P. The allocation factor for an injector is computed in a similar way. The production/injection rates that are not represented by a well (such as PSIDE, PNODE, FLUXSIDE and aquifers) are represented by I-edge and P-edge. The allocation factor for a well should add up to 1.0 (unity); but for compressible models this is not always the case, as the flow rate varies along the streamlines. The flow rate going into the bundle is not the same as the flow rate coming out of the bundle [26][36].

\subsubsection{The Governing Equations}

As a conservation of each fluid phase, the black-oil formulation is:

$$
\begin{aligned}
& \frac{\partial}{\partial t}\left(\varphi b_{w} S_{w}\right)+\nabla \cdot\left(b_{w} \vec{v}_{w}\right)=q_{w} \\
& \frac{\partial}{\partial t}\left(\varphi\left(b_{o} S_{o}+R_{v} b_{g} S_{g}\right)\right)+\nabla \cdot\left(b_{o} \vec{v}_{o}+R_{v} b_{g} \vec{v}_{g}\right)=q_{o} \\
& \frac{\partial}{\partial t}\left(\varphi\left(b_{g} S_{g}+R_{s} b_{o} S_{o}\right)\right)+\nabla \cdot\left(b_{g} \vec{v}_{g}+R_{s} b_{o} \vec{v}_{o}\right)=q_{g}
\end{aligned}
$$

Using Darcy's law, the oil, water and gas velocities vi are defined as.

$$
\begin{aligned}
& v_{o}=-\lambda_{o}\left(\vec{\nabla} P_{w}-\rho_{o} G \vec{V} D\right) \\
& v_{w}=-\lambda_{w}\left(\vec{\nabla} P_{o}-\rho_{w} G \vec{V} D\right) \\
& v_{g}=-\lambda_{g}\left(\vec{\nabla} P_{g}-\rho_{g} G \vec{V} D\right) \\
& \lambda_{i}=K \frac{k_{i}}{\mu_{i}}
\end{aligned}
$$

Where, 


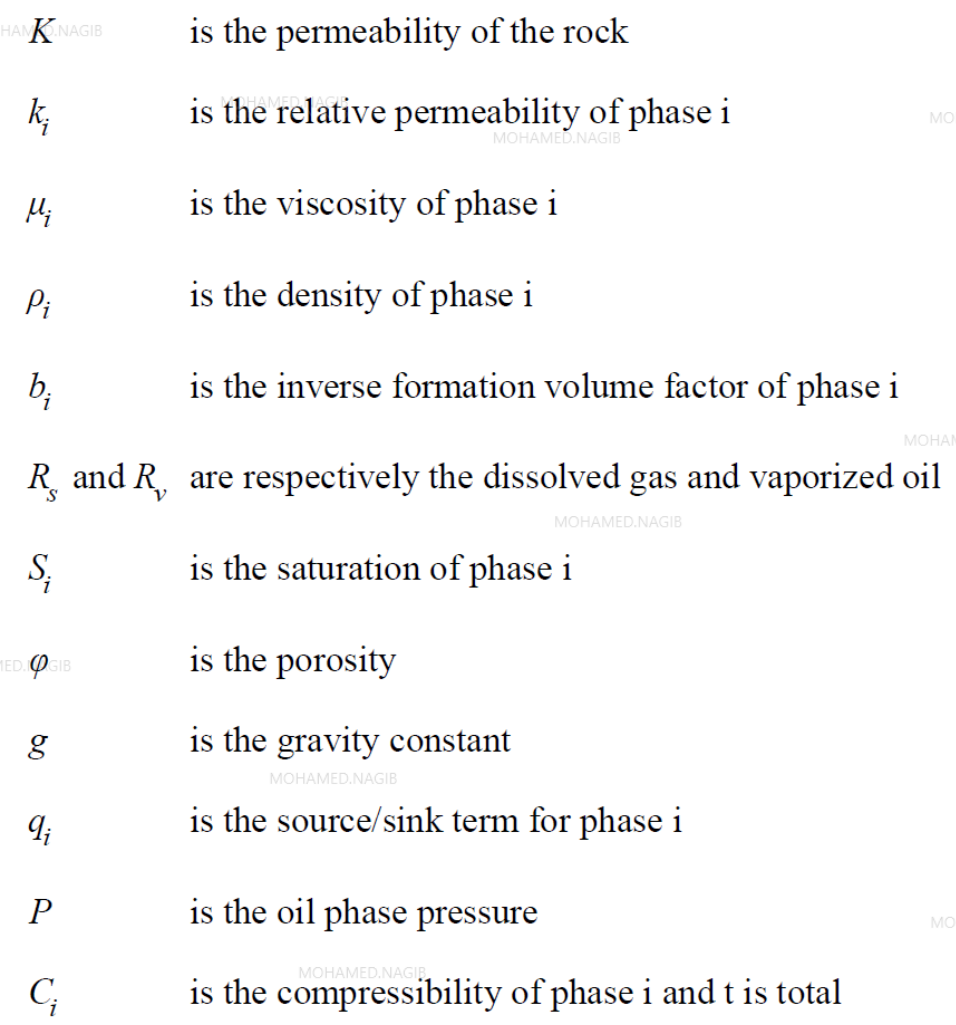

The numerical approximations of these equations generally are based on a finite-difference IMPES or fully implicit formulation. The IMPES formulation uses operator splitting to separate one equation for the pressure so that the pressure solution can be found separately from the equations modelling the movement of fluids. This approach recognizes the different nature of these equations and the potential advantage of using different numerical approaches for solving them [37].

Making the assumption, the capillary pressure $\mathrm{Pc}=0$, the pressure equation can be written as

$$
-\vec{\nabla} \cdot \vec{v}_{t}+Q=\varphi C_{t} \frac{\partial p}{\partial t}+\left(\underset{i=o, w, g}{\Sigma} \vec{v}_{i} c_{i}\right) \cdot \nabla p
$$

The total Darcy velocity i.e. $v_{t}$ is defined by $\left(\mathrm{P}_{c}=0\right)$

$$
\vec{v}_{t}=-\lambda_{t} \vec{\nabla} P_{o}+\left(\sum_{j} \lambda_{j} \rho_{j}\right) \vec{g}
$$


Equations 3.8 and 3.9 are used to find the pressure distribution for a given saturation distribution. The pressure equation is solved implicitly with a finite-difference method.

The saturation/concentration equations read:

$-\frac{\partial\left(\varphi \rho_{n} S_{n}\right)}{\partial t}+\vec{\nabla} \cdot\left(\rho f_{n}\right)=0$

Where $f$ is defined by equation 3.1

\subsection{StreamSim (3DSL)}

Another Streamline Reservoir Flow Simulator worth mentioning is that of Streamsim Technologies, Inc. Their streamline-based software uses a 4-stage simple to complex approach to manage and optimise floods by implementing these workflows: Reservoir Surveillance, Reservoir Flow Simulation, History Matching and Reservoir Sweep Optimisation.

A streamline is defined as a line that is everywhere tangent to the local velocity field at a given time. The smoke lines generated in a wind tunnel and shown in advertisements to demonstrate aerodynamic qualities of cars are a good representation of streamlines under the assumption of steady state.

Streamline simulation is characterised to be associated with six important ideas:

- Tracing 3D streamline by use of the concept of TOF rather than arc length

- Expressing the mass-conservation equations in terms of Time of flight (TOF)

- Periodic updating of the streamlines in times

- Solving the transport problems numerically along the streamlines rather than analytically

- Accounting for gravity effects

- Extension to compressible flow.

All of the above ideas originated from the need to relax the limiting assumptions inherent in the early semi-analytical stream tube methods, and adapt the method to more realistic and complex reservoir scenarios [37][38][39]. 


\subsection{Frontsim Vs StreamSim (3DSL)}

From the general comparisons and as per users experiences / feedback, the following can be summarised as an advantages of Frontsim simulator:

- Integrated with Petrel

- Petrel visualisation and plotting

- Fast

- Reduces number of full-physics simulations

- Screening geological realisations and scenarios

- Testing upscaling methods/parameters

- Runs from workflow manager

- Encourages multi-disciplinary integration

However, Streamsim 3DSL has the following features:

- Flood optimisation

- Screening of enhanced recovery projects

- Novel streamline-specific outcomes such as FLUX maps and Injection Efficiencies

- More friendly user in terms of producers injection relationships

- Automated optimiser for flood management.

From all the above the following can be summarised:

Frontsim is an excellent tool for screening geological models and work flow manager in addition to a reasonable long-term planning tools with more accurate long term production forecast. However, streamsim 3DSL is more powerful and cost effective tool for day to day optimisation.

\subsection{Conclusions}

In this chapter mathematical models were presented for both FD and SL and detailed advantages of SL in compared to FD. Fronstim and Streamsim 3DSL softwares are also presented and finally a comparison of the two simulators and differences between them. 
In summary: Frontsim is an excellent tool for screening geological models and work flow manager in addition to a reasonable long-term planning tools with more accurate long-term production forecast. However, streamsim 3DSL is more powerful and cost effective tool for day to day optimisation. 


\section{Chapter 4}

\section{SPE Comparative Solution Project}

\subsection{Description of the Original Tenth SPE Comparative Solution Model}

The model is a 2-phase (oil and gas) model that has a simple 2D vertical cross-sectional geometry with no dipping or faults. The dimensions of the model are 762 metres long by 7.62 metres wide by 15.24 metres thick. The fine scale grid size is $100 \times 1 \times 20$ with uniform size for each of the grid blocks. The top of the model is at 0.0 metres with initial pressure at this point of 100psia. The model in the beginning is fully saturated with oil (no connate water). The permeability distribution is a correlated geo-statistically generated field. The fluids are assumed to be incompressible and immiscible. The fine grid relative permeability capillary pressure was assumed to be negligible in this case. Gas was injected from an injector located at the left of the model and dead oil was produced from a well on the model. Both wells have a well internal diameter of 1.0ft. and are completed vertically throughout the model. The injection rate was set to give a frontal velocity of $0.3 \mathrm{~m} / \mathrm{d}$ (about 1 foot/day or $6.97 \mathrm{~m}^{3}$ per day), and the producer is set to produce at a constant bottom pressure limit of 15,000psia. The reference depth for the bottom hole pressure is at the top of the model. The tasks specified were to apply upscaling methods in the following scenarios:

1. $2 \mathrm{D}-2 \mathrm{D}$ uniform $5 \times 1 \times 5$ coarse grid model

2. 2D - 2D non-uniform coarsening, max 100 cells, directional pseudo-relative permeabilities were allowed if necessary [25].

The SPE comparative solution presented two key problems: a gas injection study and waterflood of a large geostatic model. Nine participants provided results for one or both problems and the results are presented in this work. The main purpose is to rate the best simulator by comparing respective speed and visualising accuracy. The research consortia who participated in this study and the associated simulators used are shown in Table 4-1. 
Table 4-1: Table showing Participants

\begin{tabular}{|c|c|c|c|c|c|c|c|}
\hline Chevron & Coats & Geoquest & Landmark & Phillips & StreamSim & Total & Roxar \\
\hline Cheers & SENSOR & Frontsim & VIP & SENSOR & 3DSL & Eclipse & RMS \\
\hline \multicolumn{8}{|l|}{ UNSW } \\
\hline IMEX & & & & & & & \\
\hline
\end{tabular}

The fluid description of model 1 is a two-dimension oil and gas vertical cross-section dipping fault with areal dimension $2500 \mathrm{ft}$. by $25 \mathrm{ft}$. and the model's thickness is $50 \mathrm{ft}$ with a $100 \times 1 \times 20$ grid size. Model 1 is initially saturated with oil and has average initial pressure of 100 psia.

The fine grid relative permeability has a residual oil saturation of 0.2 and critical gas saturation of 0.0 , capillary pressure is negligible and gas was injected from an injector at the left flank of the reservoir, which led to production of under-saturated oil from a well at the right flank of the reservoir. The producer and injector are vertically completed with the same diameter, with the producer producing at a constant pressure limit of 95 psia. Figure 4-1 shows the permeability distribution in the model and Figure 4-2 shows the 3D Porosity model of fine grid channel sand.

There are four vertical producers located at the four corners of the model and completed throughout the formation. Each of these producers flow at $4000 \mathrm{psi}$ bottom hole pressure, central injector exists at an injection rate of $5000 \mathrm{bbl} /$ day at maximum injection bottom hole pressure of 10,000psi.

For the relative permeability of oil and water, Equations 4.1 and 4.2 were the ones generally used for calculating these. However, Figure 4-1 showed the relative permeability curve generated from Corey's Model. Table 4-2 showed the PVT Table of under saturated black oil table.

$$
k_{r w}=\left(\frac{S-S_{w c}}{1-S_{w c}-S_{o r}}\right)^{2}
$$


$k_{r w}=\left(\frac{1-S-S_{o r}}{1-S_{w c}-S_{o r}}\right)^{2}$

$S_{w c}^{2}=S_{w i}=S_{o r}=0.2$

Assuming $S_{o r}=0.2$, the relative permeability curve is constructed as shown.

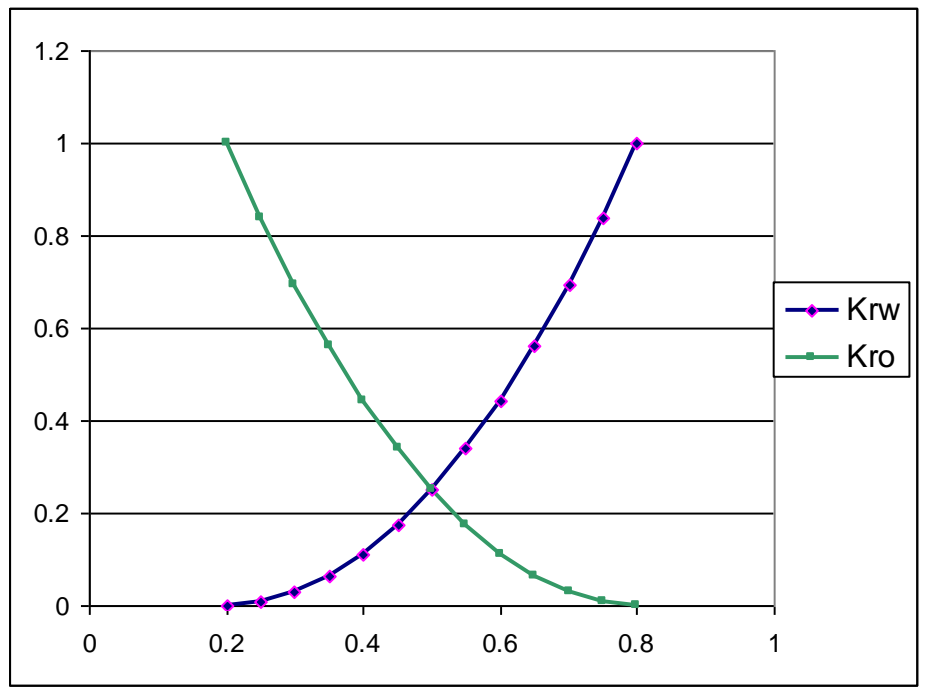

Figure 4-1: Relative permeability curve generated from Corey's model, with both $\mathrm{K}_{\mathrm{rw}}$ and $\mathrm{K}_{\mathrm{ro}}$ exponents equal to 2 .

Table 4-2: PVT property of under saturated oil used in model 2.

\begin{tabular}{|c|c|c|}
\hline $\mathrm{P}(\mathrm{psi})$ & Bo (rb/STB $)$ & $\mu_{\mathrm{o}}(\mathrm{cp})$ \\
\hline 300 & 1.05 & 2.85 \\
\hline 800 & 1.02 & 2.99 \\
\hline 8000 & 1.01 & 3.0 \\
\hline
\end{tabular}




\subsection{Objective}

The technical concept of the Tenth SPE comparative study is to apply different upscaling techniques in the two problems discussed previously. However, for this project work, we shall look at the impact of streamline simulation in the results.

The objective of the Tenth SPE comparative study is to upscale the $60 \times 220 \times 85$ geostatistical model to a coarse grid and compare results generated on both fine and coarse grid using core and pseudo-based relative permeabilities.

\subsection{Enhancement work on the Tenth SPE model}

A review of the contribution of this thesis to the Tenth SPE comparative model follows the simulation of the upper zone (Tarbert of the fine grid model). The upper Tarbert has a grid size of $60 \times 220 \times 35$, areal dimensions are the same as the full field model and the vertical extent for the fine grid ranges from layer 1 to layer 35. The upscaling method used in the SPE model is applied in the fine grid case to reduce the upper zone to not more than 15,000 cells. With the assumption that flow to the well is uniform across the completed interval, the reservoir volume injection rate was slightly reduced to a rate that corresponds to the impact of the perforation interval of Tarbert zone in the full field model. The completion interval for the wells is shown in Figure 4-2. 


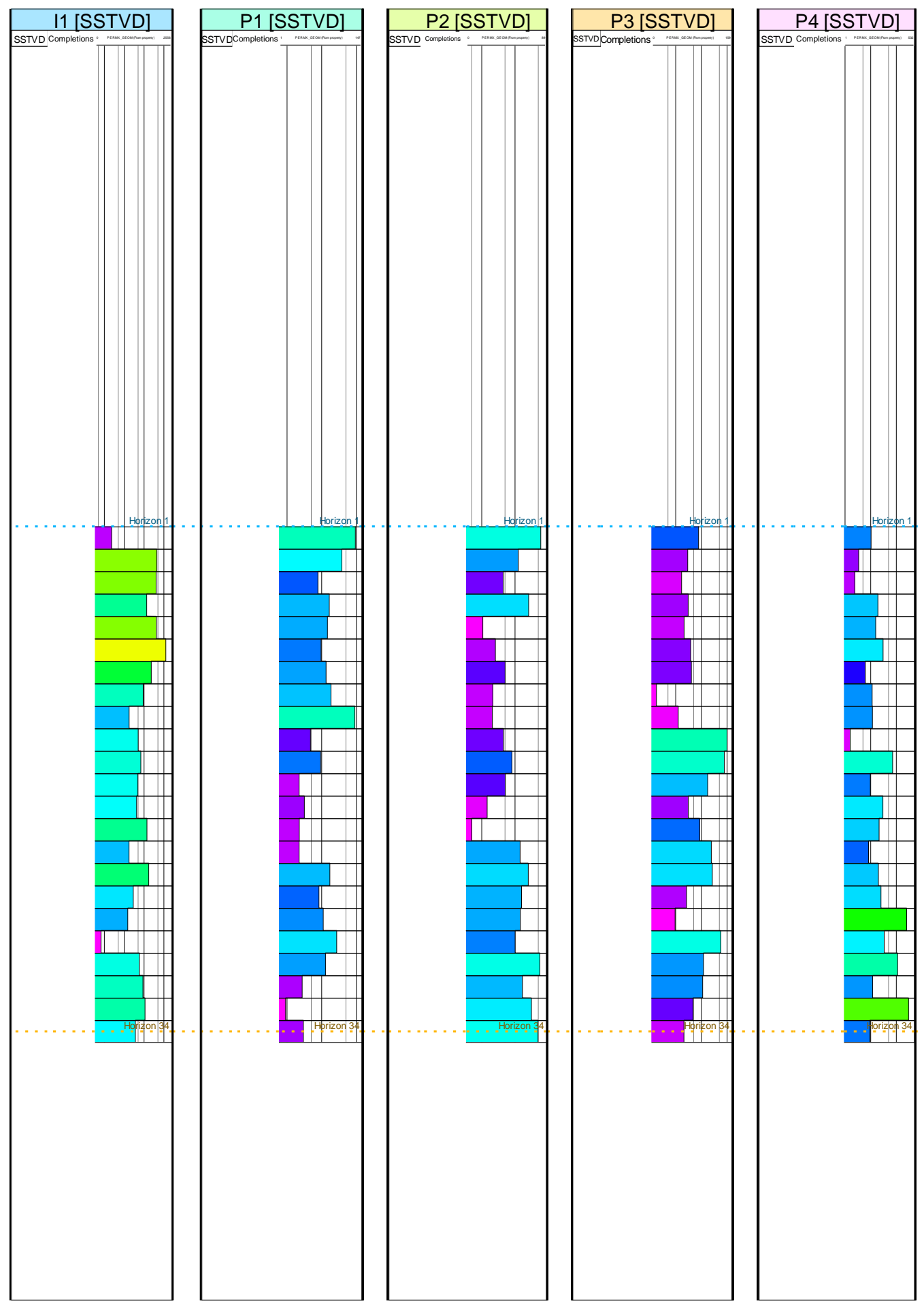

Figure 4-2: Well section showing completion interval for all the wells 
The fine prediction results and the relative permeability of the full field models were used to history match the upscaled model.

In the making of the upscaled model, the top and bottom surfaces (i.e. the 1st and 34th layers) were used as constraints to make a $19 \times 33 \times 24$ grid in Figure $4-3$. During the upscaling process, the fine porosity was upscaled using both arithmetic and harmonic methods. Geometric permeability upscale was used for permeability upscaling.

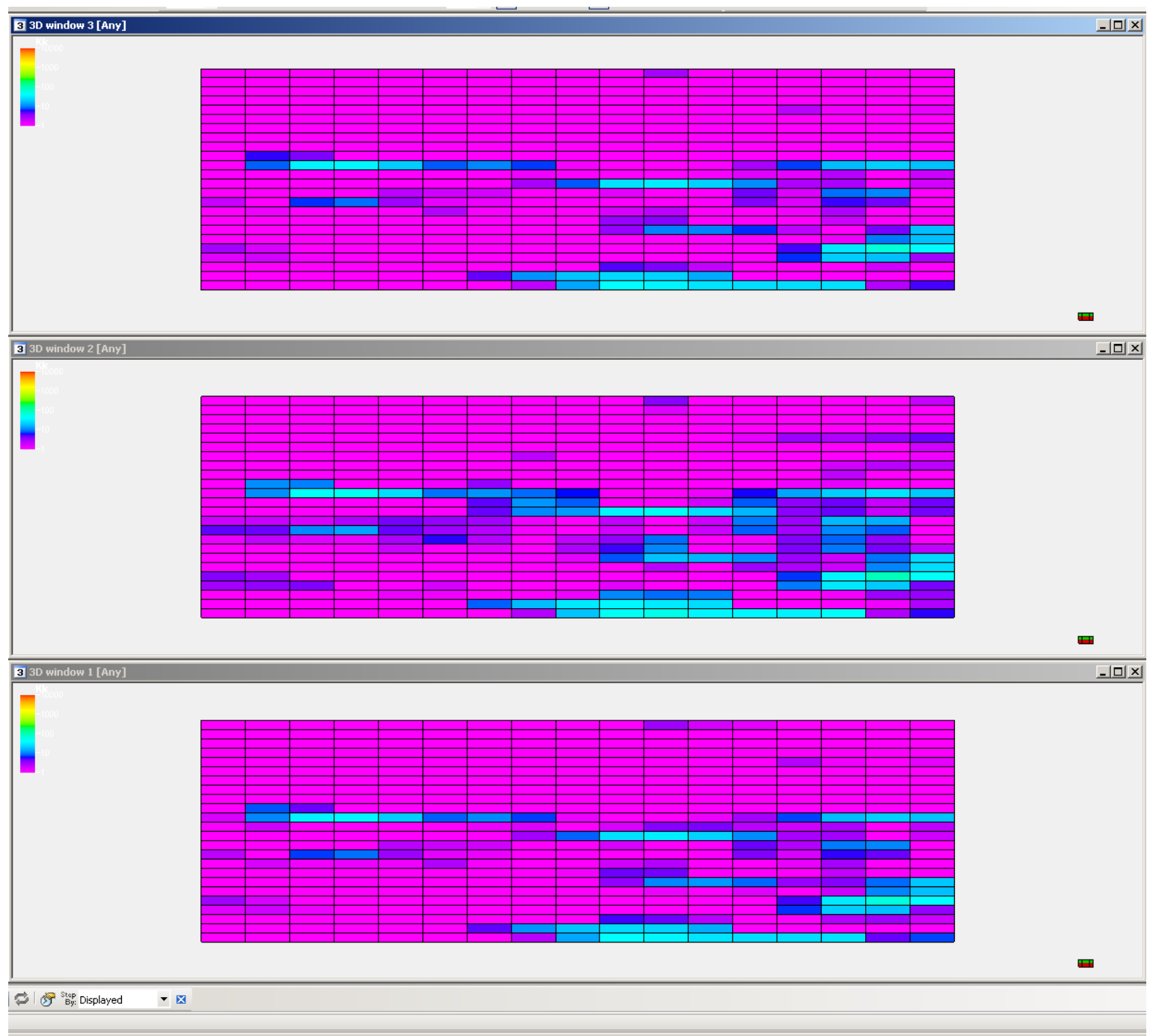

Figure 4-3: Different vertical permeability upscale for upper Tarbert 
This is valuable information used to check exactness of upscale when the resulting correlation is matched with core analysis porosity-permeability cross plot as shown in Figure 4-4.

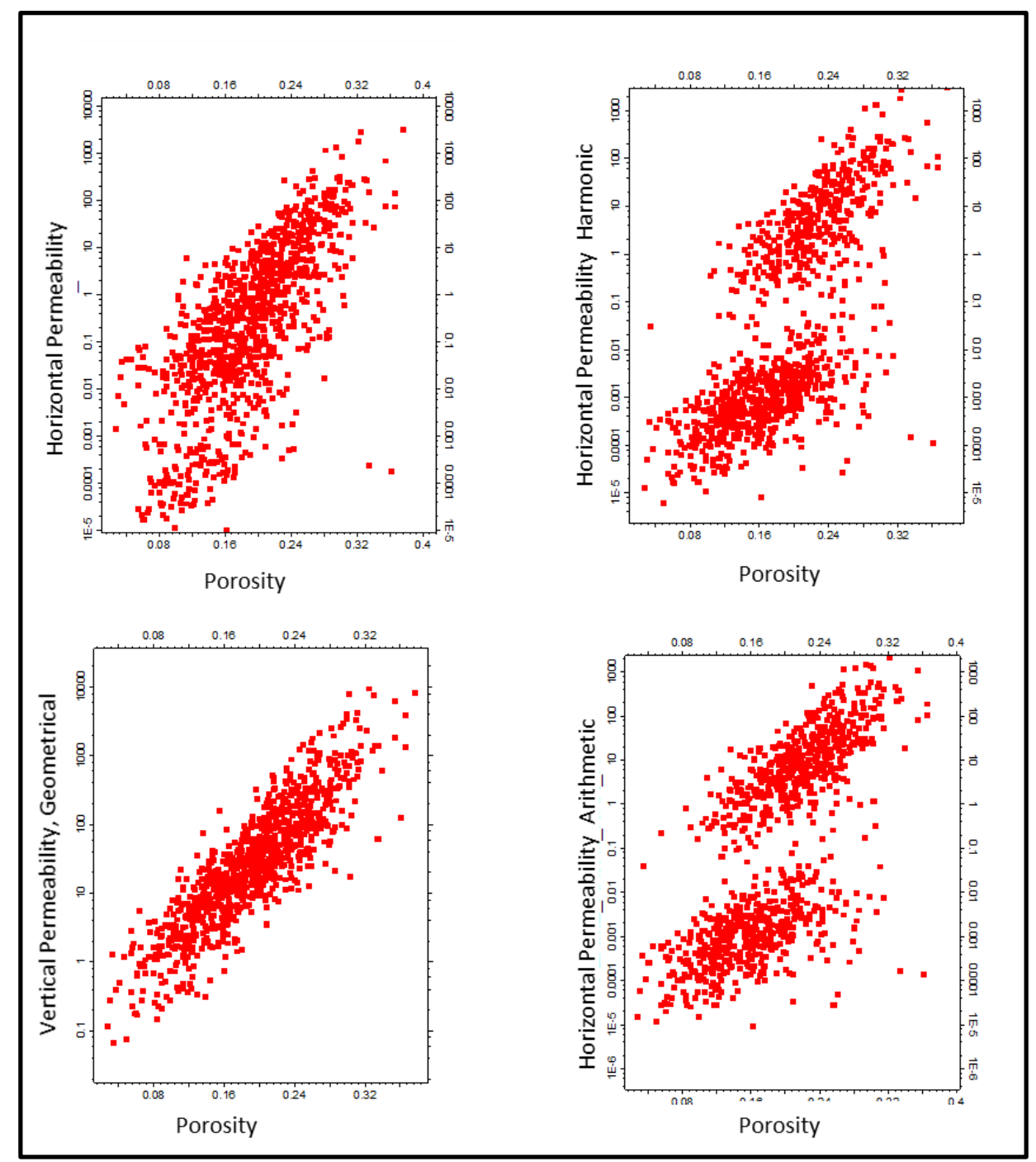

Figure 4-4: Investigating petrophysical relationships for upscaled properties

Figure 4-5 shows well production performance of one the four producers. As can be seen green colour is oil production rate for the first producer, initially started with constant plateau rate then start declining after approximately 4 months, water cut in blue colour and water breakthrough started after 
about 6 months. Hence, water cut started to developed gradually till reach $95 \%$ at the end of simulation time.

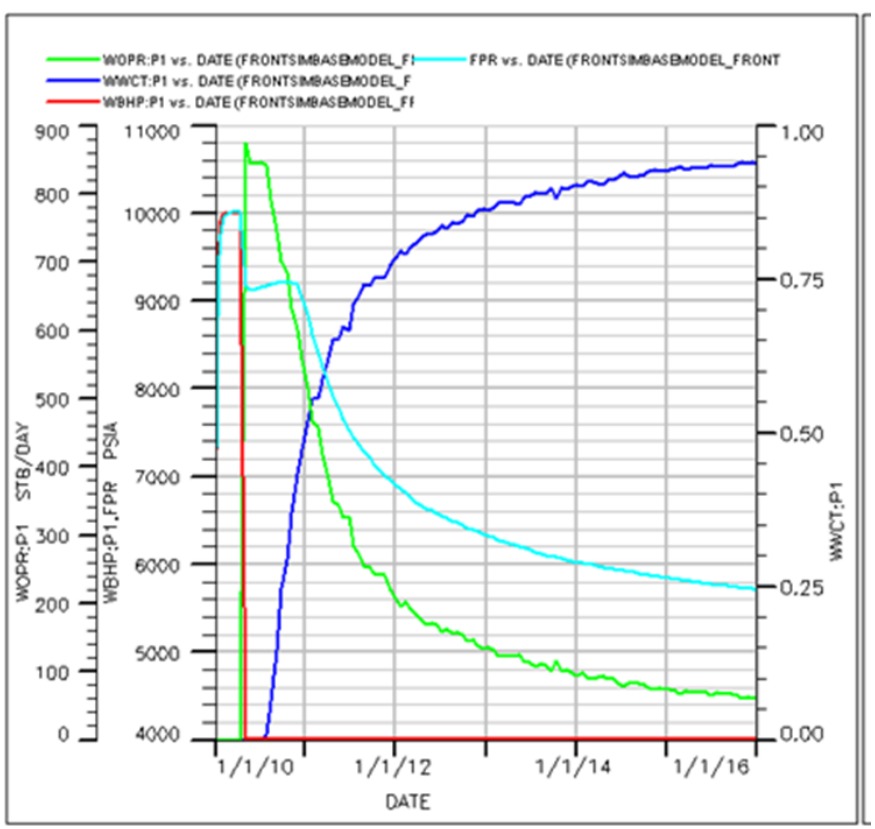

Figure 4-5: Showing Water cut, Oil Production and Bottomhole Pressure for all the producers.

Figure 4-6 shows the streamlines generated from injector to the four producers at the first timesteps.

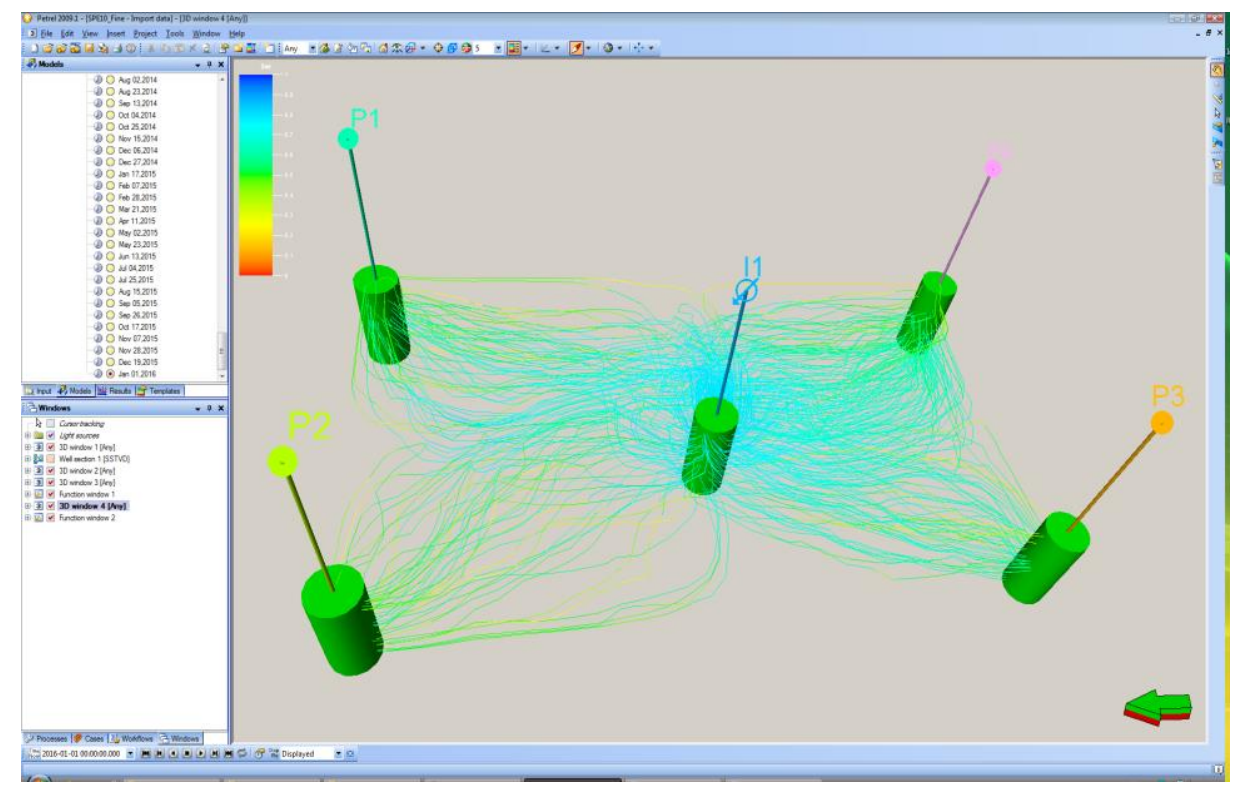

Figure 4-6: Streamlines for water injection for the first-time step. 


\subsection{Conclusion}

In this chapter, a detailed discussion for all the previous work has been done using the Tenth SPE Comparative solution project using water injection in both fine and coarse grids. Upscaling techniques and petrophysical data inputs also presented in addition to the predicted well performance and streamline generated by the water injector

In the next sections, SL models will be converted to simulate different recovery schemes in order to test the gas displacement process 


\section{Chapter 5}

\subsection{Research Methodology and Simulation Models}

To achieve the objectives of this research, several injection methods were tested, such as water injection, water alternating gas and gas injection using Eclipse 100 and FrontSim as the basis for comparison, then compositional modelling to include the compressibility effect. The main objective is to quantify the differences between finite difference and streamline simulations, especially for gas injection as the technology has already been proved for water flood.

Simulation results were analysed, graphs were generated and interpreted, and subsequently results were compared. The results were monitored noting the relative changes in quantities such as time steps, total volume of production, rates of production at each producer, varying rates of injections, etc.

This chapter will present the three modelling cases to prove the theory of streamline simulation. The testing of streamline technology for a gas displacement process will be presented in more detail in a separate chapter.

\subsection{Models Description used for the Research}

The model used for this investigation was developed from the original Tenth SPE Comparative Solution project (Christie and Blunt, 2001). The Original Tenth SPE Comparative Solution Model was developed with a Dead Oil PVT structure. The model consists of a 5-spot pattern (i.e., four producer wells placed at each corner of the reservoir and one injector at the centre). The model has a simple geometry, with no variation in the top structure and no faults. The model is described on a regular Cartesian grid within the fine geological model scale with $15 \times 55 \times 17$ (14025) grid cells and it consists of two formations: Shallow-marine Tarbert formation in the top 10 layers, where the permeability is relatively smooth, and a fluvial Upper-Ness in the bottom. The two formations contain large permeability variations, approximately 10 orders of magnitude as can be seen in Figure 5-1. In addition, the most heterogeneous structure lies in the Upper-Ness formation. The model was adapted for a different secondary recovery technique, e.g. Water Injection, Water Alternate Gas (WAG) and Gas Injection, as per the objective. All models have the same number of grid cells, namely 14,025 cells as described earlier, PVT, rock properties, and fluid densities. For the case of gas injection, the model becomes a three-phase run (i.e. water, gas and 
oil phases). Since we consider a three-phase fluid description for gas injection, live oil (i.e. dissolved gas with oil) was used. Well completion and operation constraints for the four different models remain the same, with simulation periods over the same duration of time. Figure 5-1 represents a 3D Model with the permeability distribution and well configurations as for the Tarbert formation. Four oil producers and one injector comprise one 5-spot pattern, all the four producers are completed in all vertical grid cells. For the case of the Gas Injection model, 4 phases were considered in the simulation: Oil, Gas, Dissolved Gas for gas injection and Water. All grid cells have the following dimensions: $80 \mathrm{ft}$. in $\mathrm{X}$ direction, $40 \mathrm{ft}$. in $\mathrm{Y}$ direction and $10 \mathrm{ft}$. in $\mathrm{Z}$ direction as height and this will compromise the oil in place in the model. This results in pore volume 13.7 reservoir barrels and oil in place 6.8 million stock tank barrels (STB).

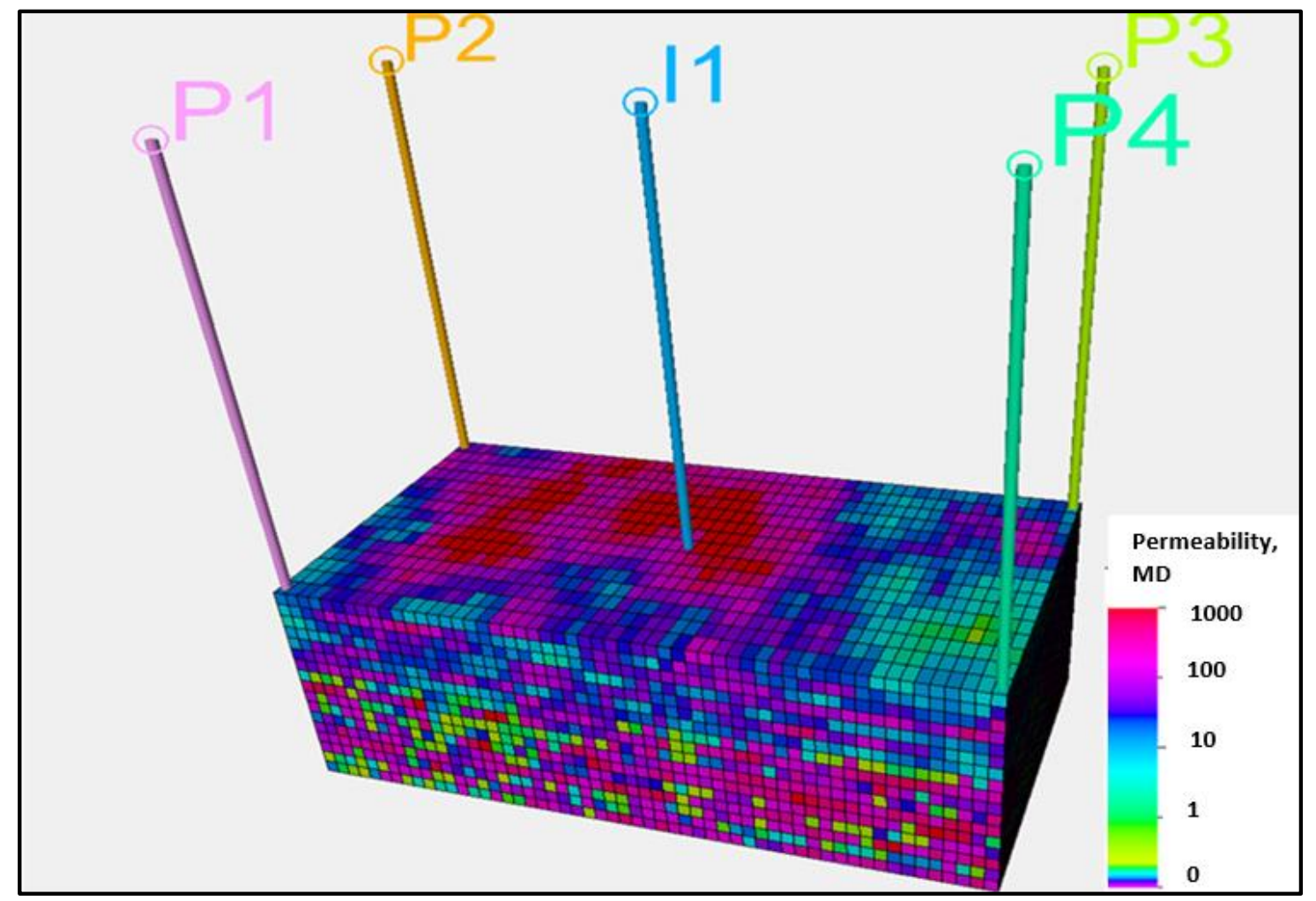

Figure 5-1: 3D Permeability Distribution and Well Configurations for the Tarbert Formations. 


\subsubsection{Water Flooding Model}

In this case, the model was developed to improve the production of this heterogeneous reservoir using water injection. Having observed the steady depletion of production of the four producers over a 6 years production period considering the nature of the reservoir, being more of a water drive reservoir, a probable solution was to adopt the water flooding or water injection as the first measure to tackle the problem of improving the oil production.

\subsubsection{Water Flooding Model Analysis}

The following grid view shows the oil saturation to show the progression and the direction of sweep towards the producers. Figure 5-2 shows the oil saturation grid for water flood FD and SL Models. As can be seen in the graph, the upper two graphs show the FD simulation and the bottom two graphs the SL simulation model. Good agreement is shown for the oil saturation grid at both the first- and last-time steps.

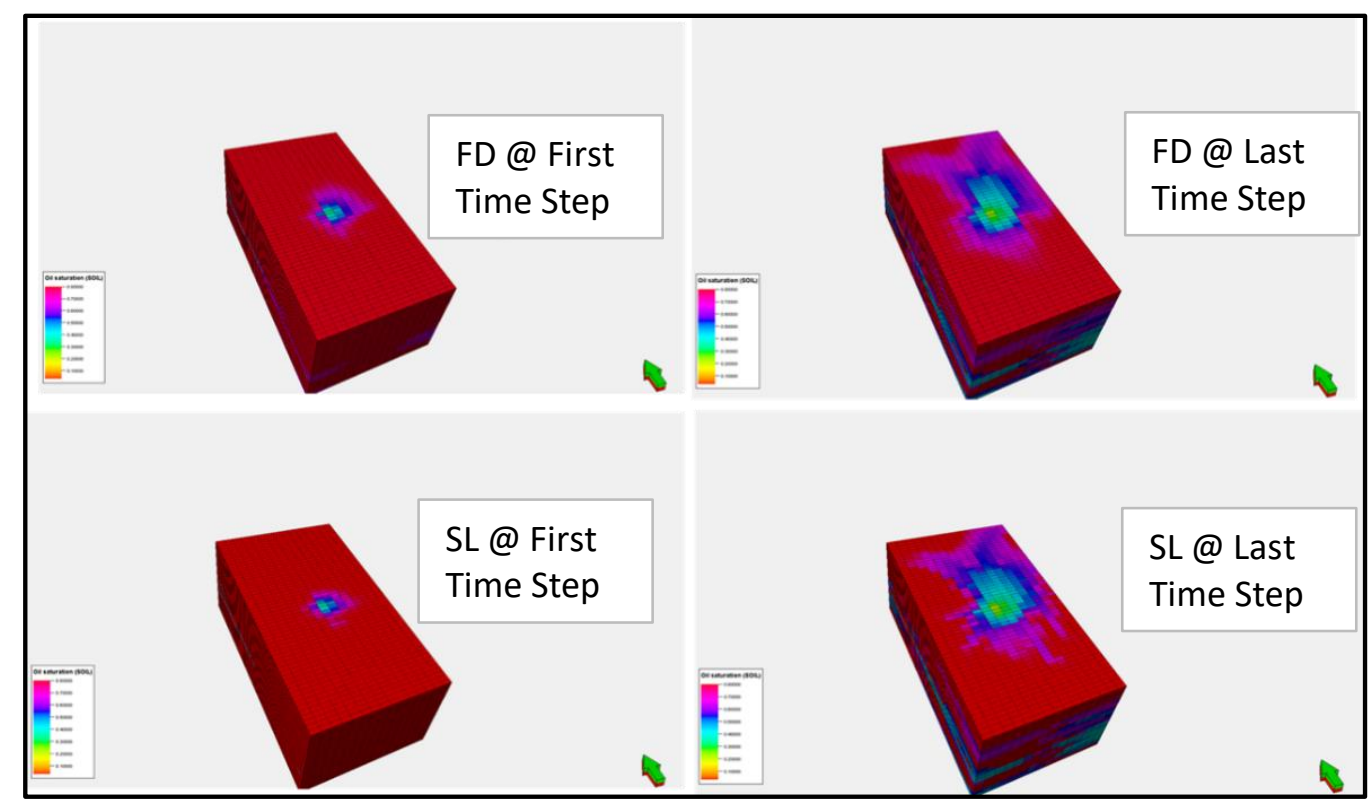

Figure 5-2: Oil Saturation Grid for water flooding at Different Time Steps

\subsubsection{Water flooding Model Performance}

A detailed analysis and comparison of both FD and SL simulation models are presented in this section. SL technology has already been proved and used now for decades in modelling and optimisation of water flood projects. So, less relative errors and differences were expected to be seen from these results. In the following section, detailed 2D graphs will be presented for a detailed comparison of all vectors 
calculated by both FD and SL simulation models. Figure 5-3 shows a comparison of the oil production rates of both FD and SL simulation models. A good match is obtained for time later than 1st Jan 2009. Minor differences are noticed at times earlier than this as a result of the differences in time steps between FD and SL models.

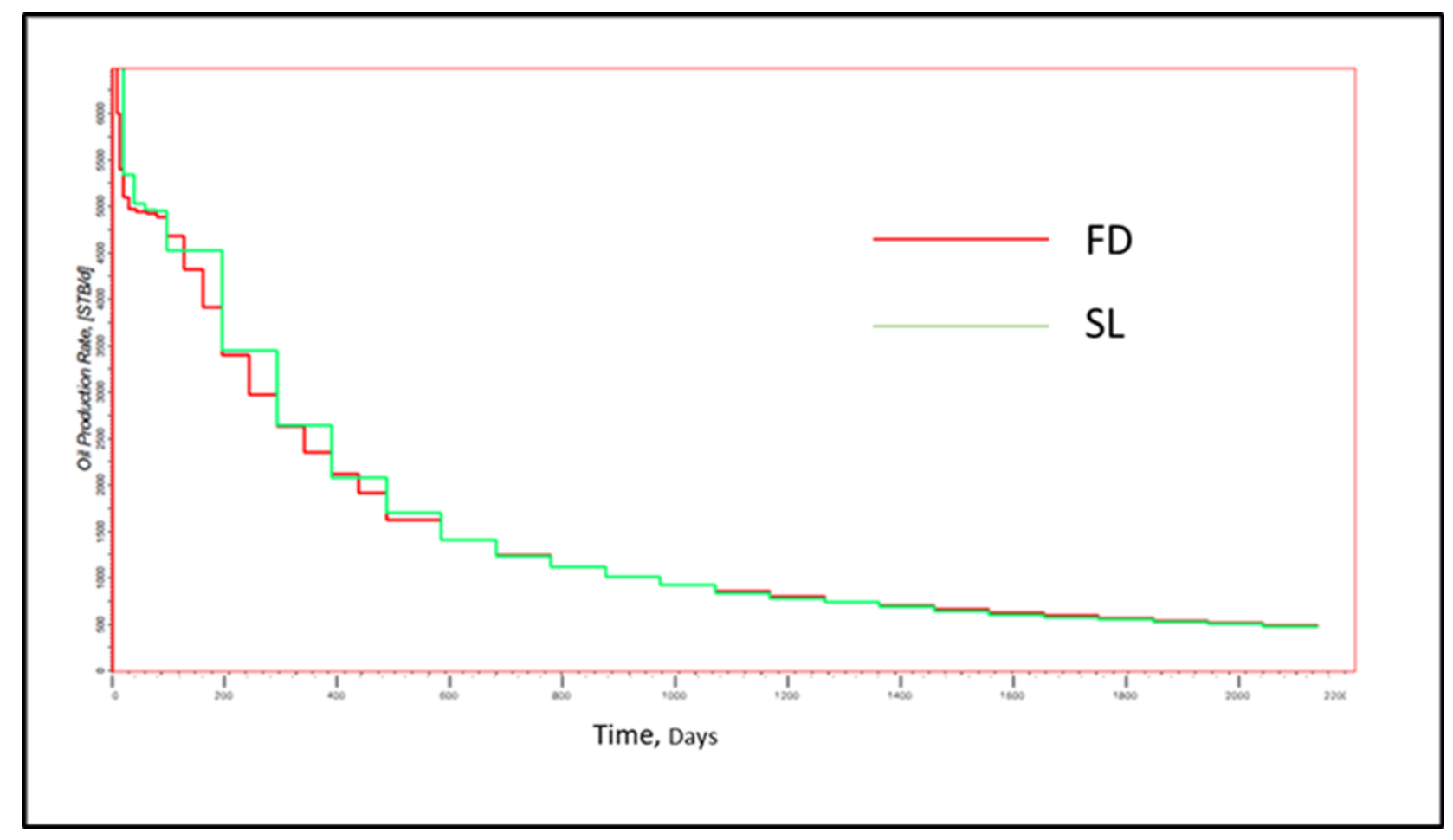

Figure 5-3: Comparison of Oil Production Rates of FD and SL Water Flood Models

Figure 5-4 shows a comparison of the total oil production of both FD and SL simulation models. A good match can be seen, with no major differences of the time steps required in both models. The results of total oil production shows that a very small difference was achieved, with SL slightly larger than FD. 


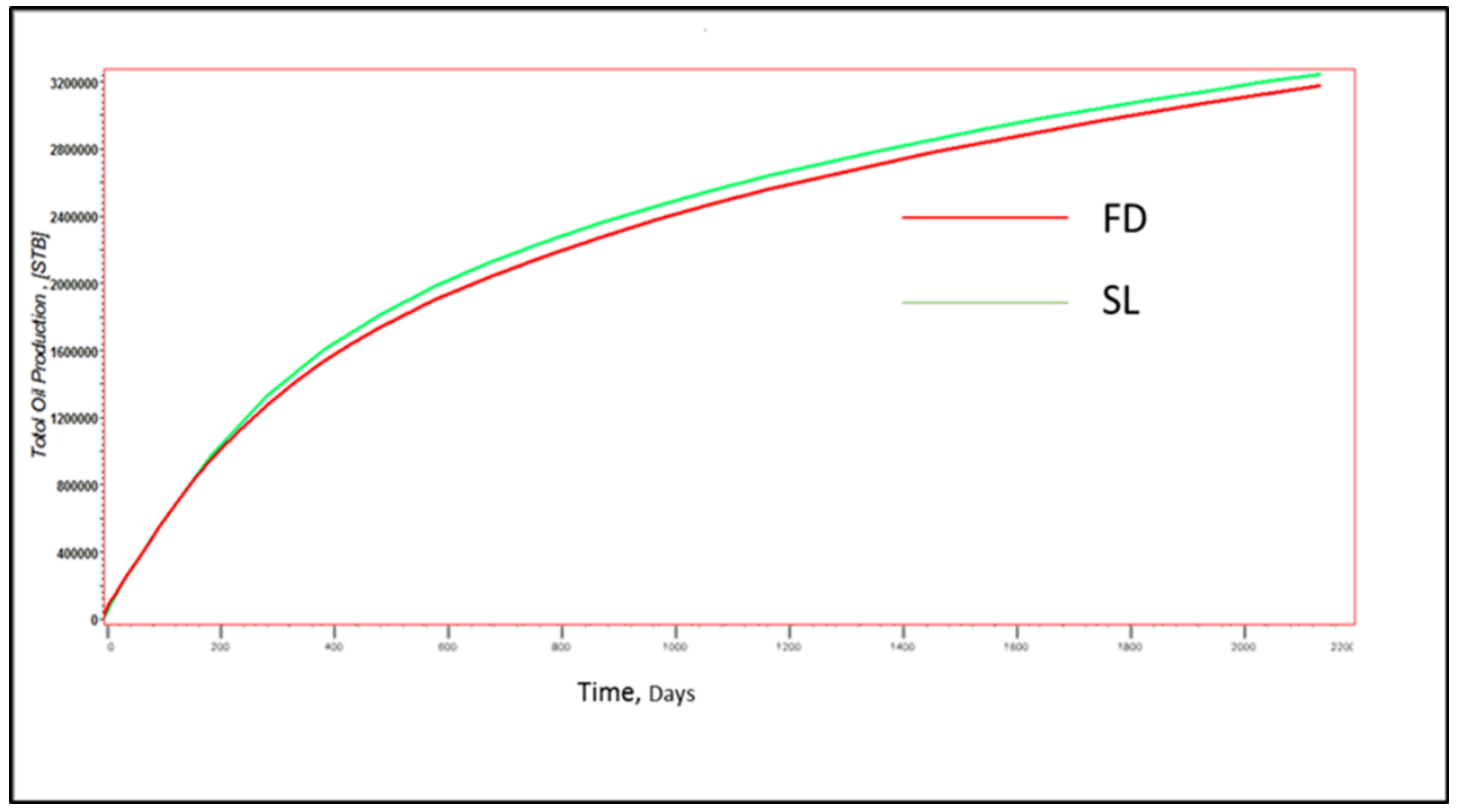

Figure 5-4: Comparison of total Oil Production of FD and SL Water Flood Models

Concerning the water production profiles comparisons. Figure 5-5 shows the water production profiles for the water injection model for both SL and FD models. A good match is obtained for time later than 1st Jan 2009.

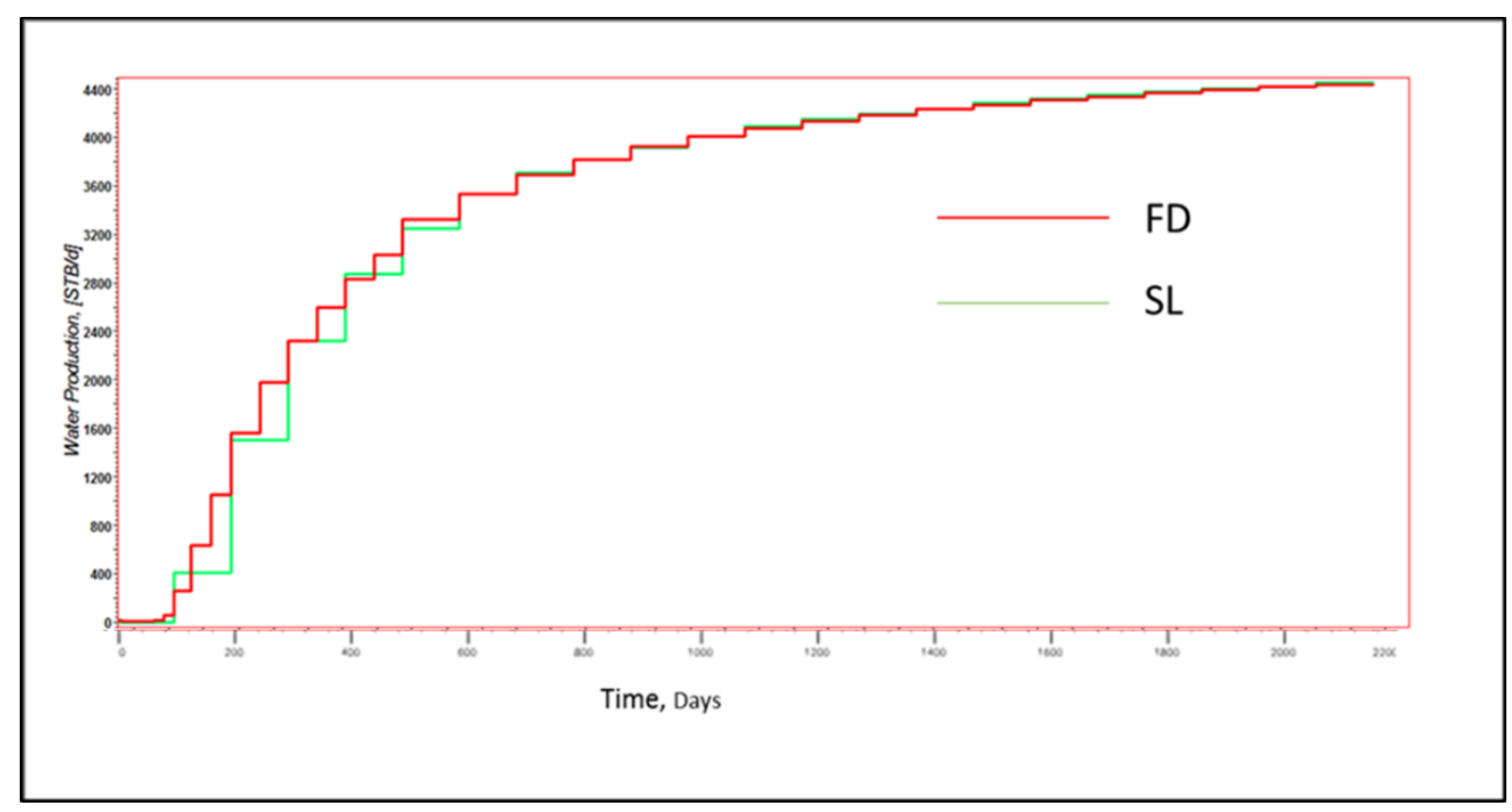

Figure 5-5: Comparison of Water Production Rates of FD and SL Water Flood Models 
Water cut performance for both models are presents in Figure 5-6 and only small differences are noticed from the graph.

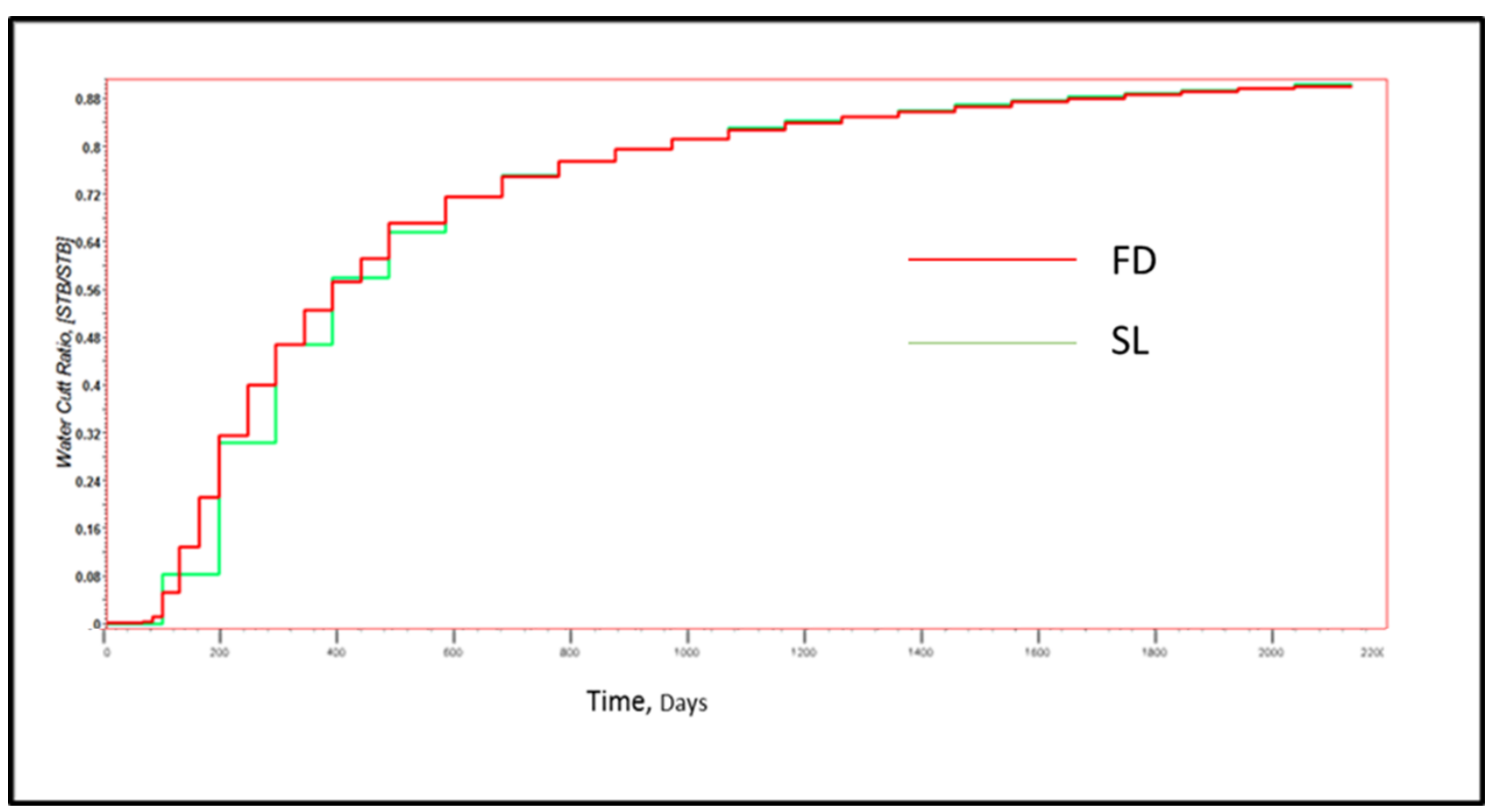

Figure 5-6: Water Cut Performance for Water flooding Model

Comparison of the pressure performance is presented in Figure 5-7 for both FD and SL simulation models of the water flood case. As can be noticed in the graph both pressure trends are in line with a minor difference indicating the high accuracy of SL simulation model of the water flood case. 


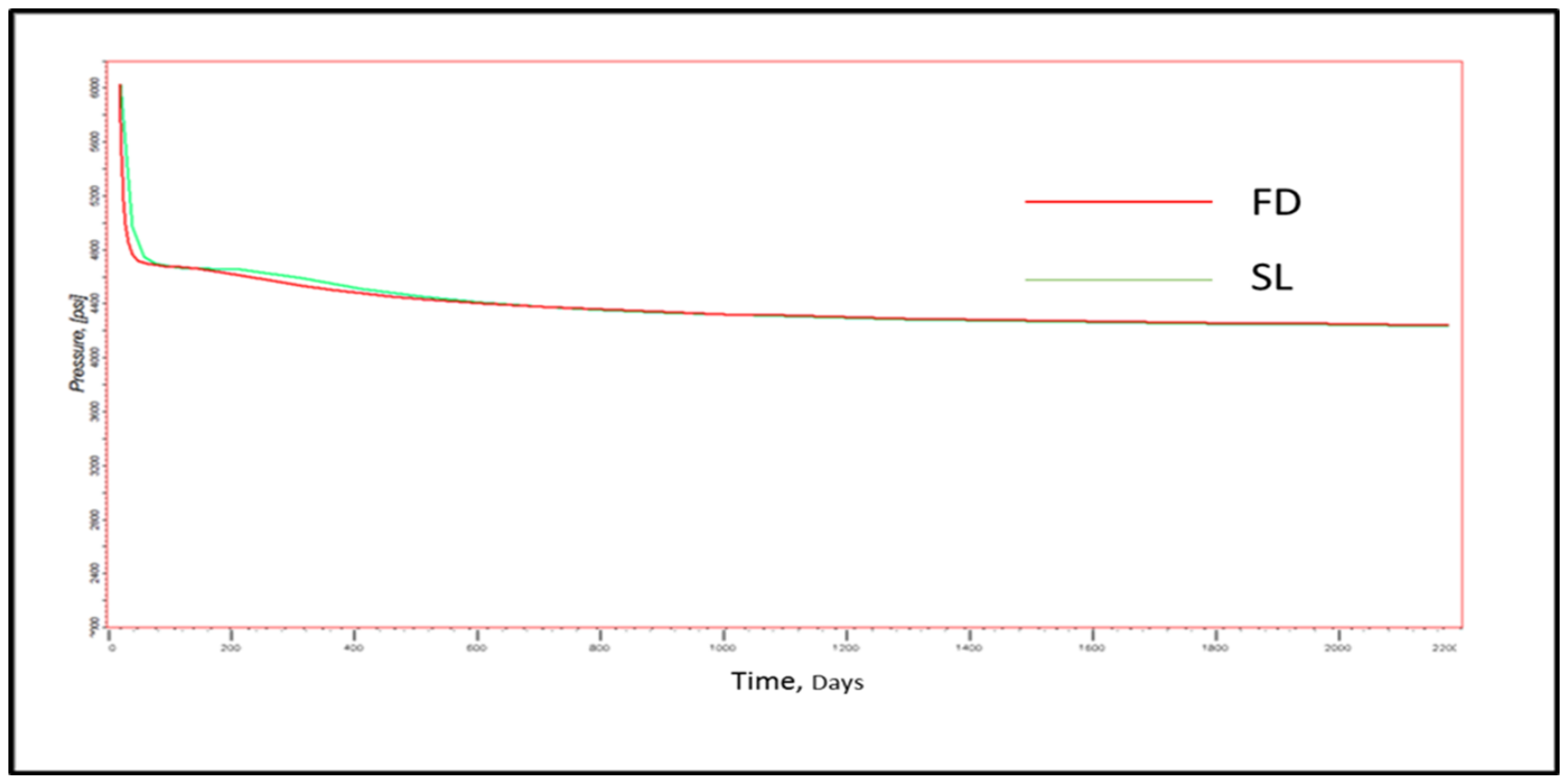

Figure 5-7: Comparison of Pressure Performance of FD and SL Water Flood Models

In order to cover all the controlling parameters/factors affecting the simulation performances of both FD and SL simulation models, in this section detailed comparisons will be presented of the following quantities:

- Total CPU used for FD vs SL

- Detailed distribution of CPU required and used for each solver on the SL simulation model

- Detailed numbers of Newton iterations used by FD vs SL

- Detailed numbers of Linear iterations used by FD vs SL

- Total number of streamlines used in the SL model

Figure 5-8 represents the total CPU required to complete the simulation of both FD and SL models. As indicated in the graph, almost double the amount of CPU is consumed for the FD simulation model in comparison to the SL simulation model. 


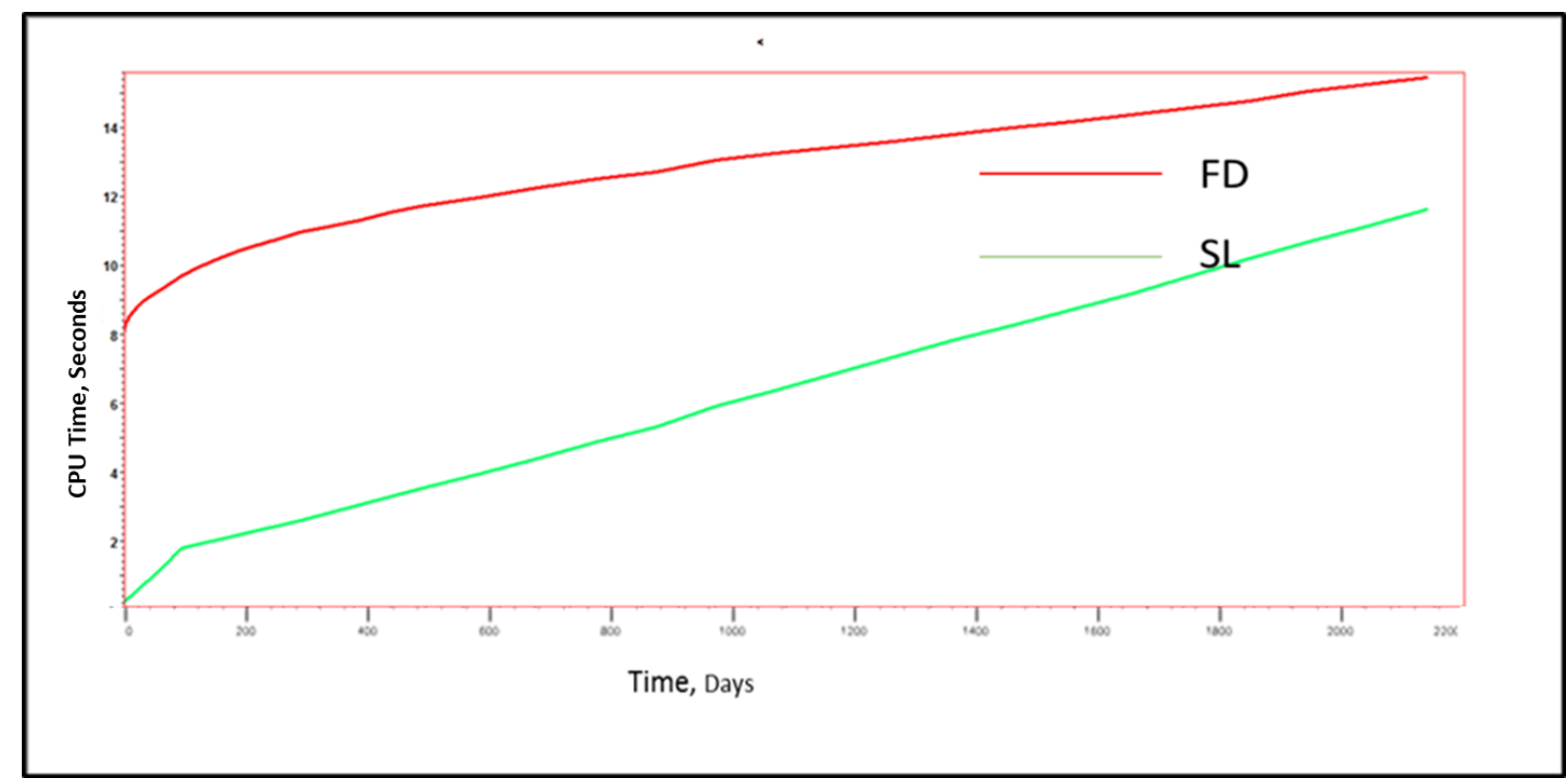

Figure 5-8: Total CPU Comparisons for FD and SL Models

Figure 5-9 represents the detailed CPU time distribution in order to complete the simulation run. As can be seen from the graph, the highest amount of CPU time is taken by the saturation solver, followed by pressure solver. Assembling the matrix as both takes same amount of CPU time. Both initialization and velocity solvers employ the lowest amount of CPU time.

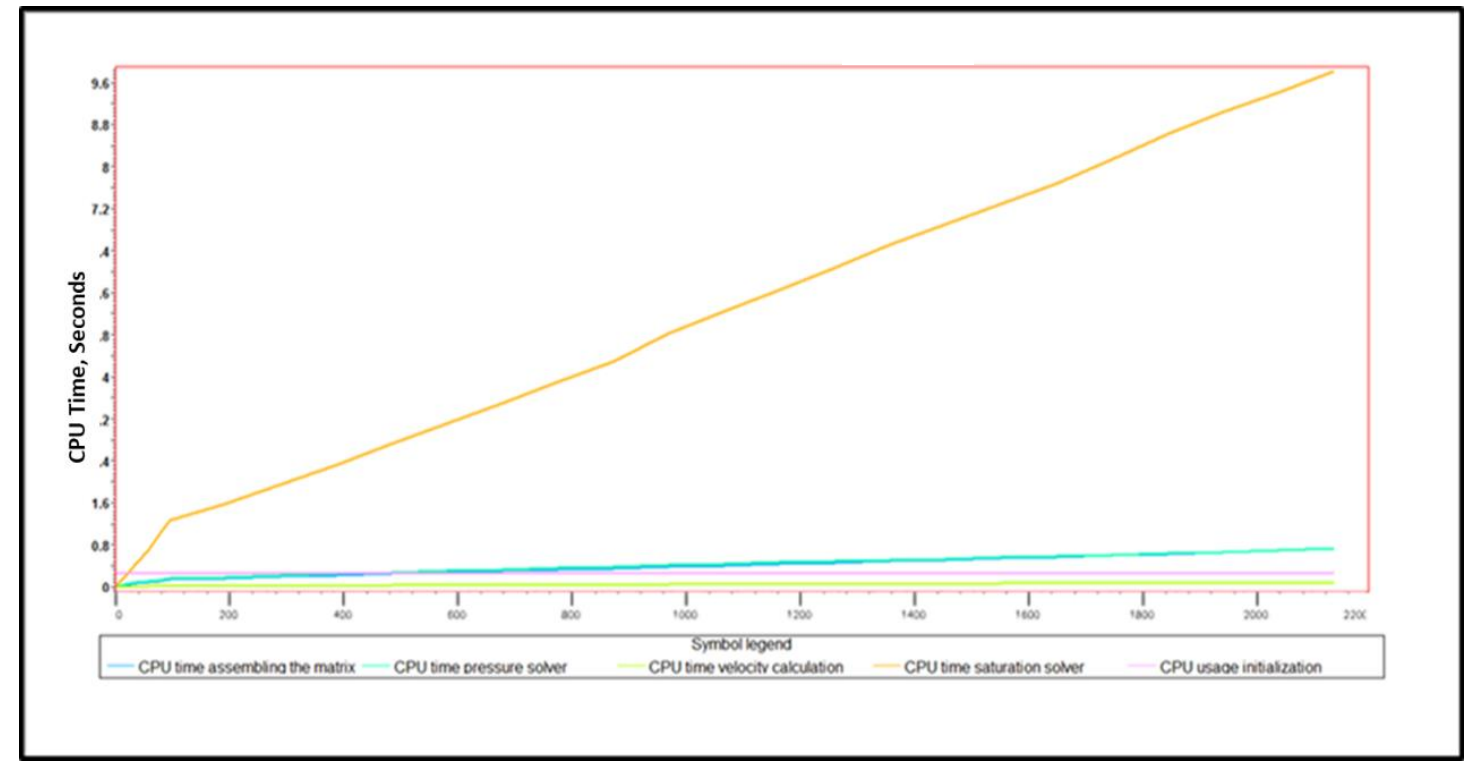

Figure 5-9: CPU Time Required for Different Solvers used for SL Calculations 
Figure 5-10 represents a comparison of the number of Newton iterations used for FD vs SL simulation models. As can be seen, the SL simulation model uses a lower number of Newton iterations, and is more stable in comparison to the FD simulation model.

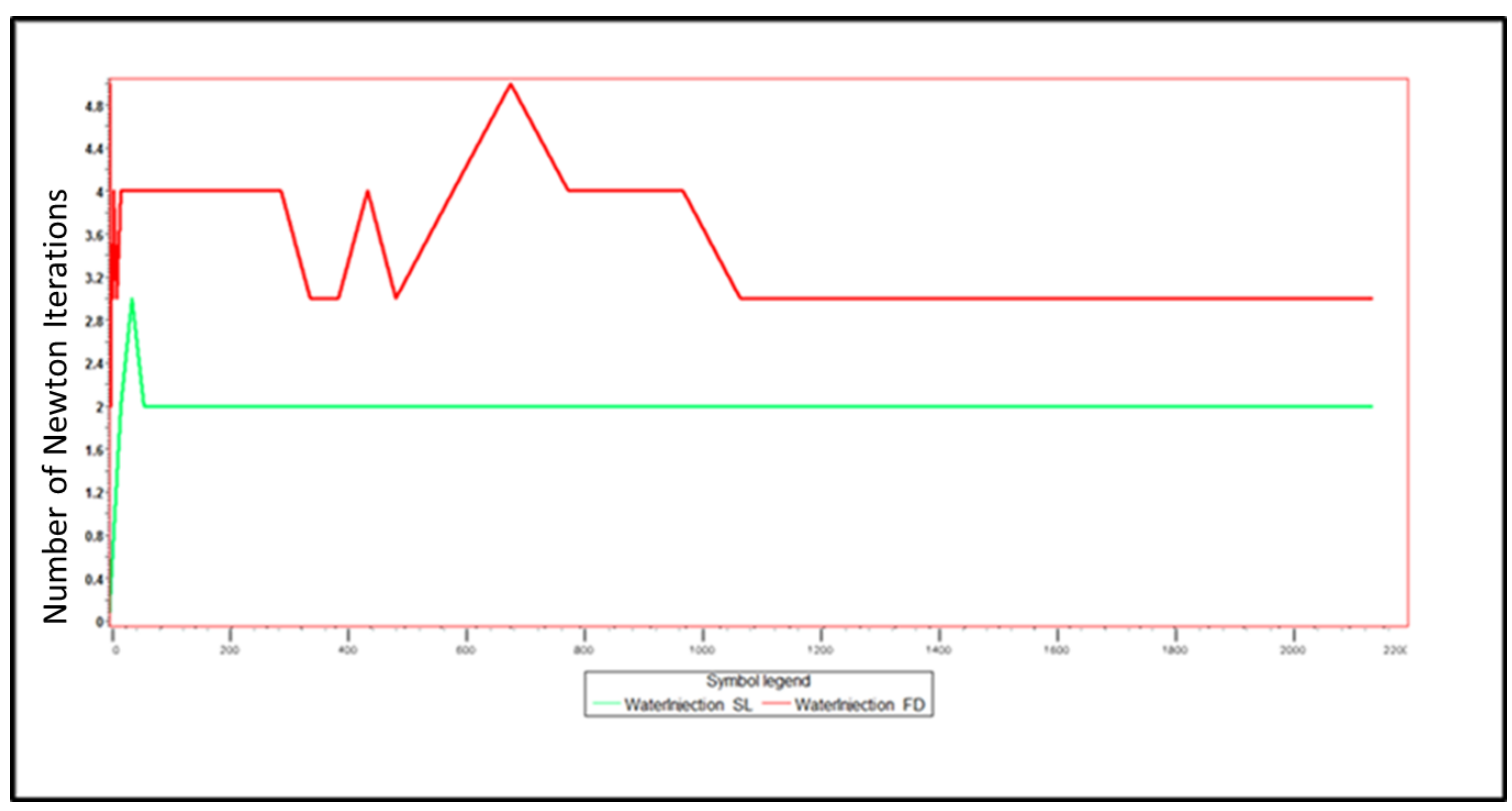

Figure 5-10: Comparisons of Number of Newton Iterations Calculated for FD and SL Models.

The same observation can be concluded as well from Figure 5-11 for the linear iterations of FD vs. SL simulation models as well.

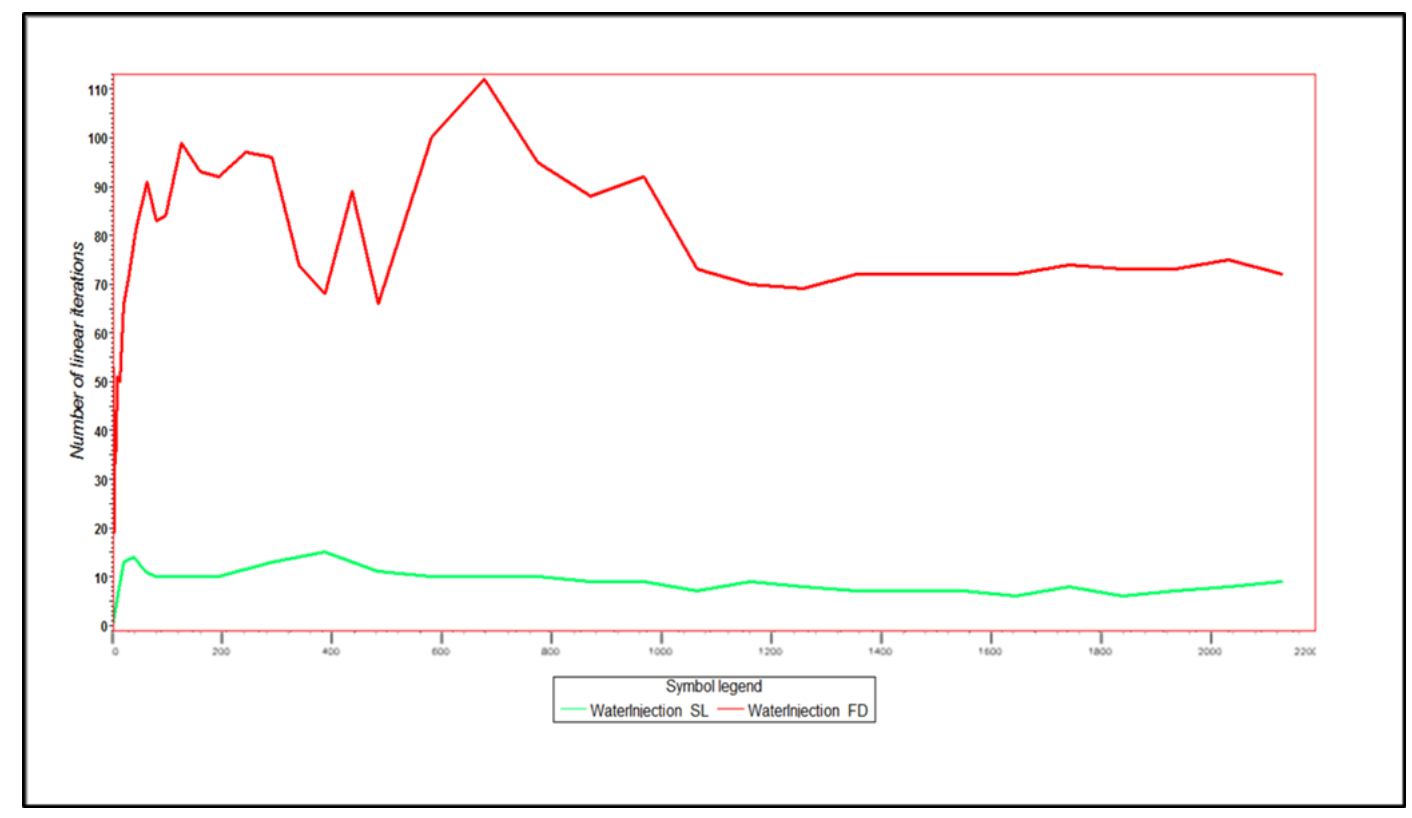

Figure 5-11: Comparisons of Number of Linear Iterations Calculated for FD and SL Models 
The number of streamlines required on the water flooding model is presented in Figure 5-12. As can be seen from the graph, 3000 streamlines were generated to calculate the time of flight and to map the saturation.

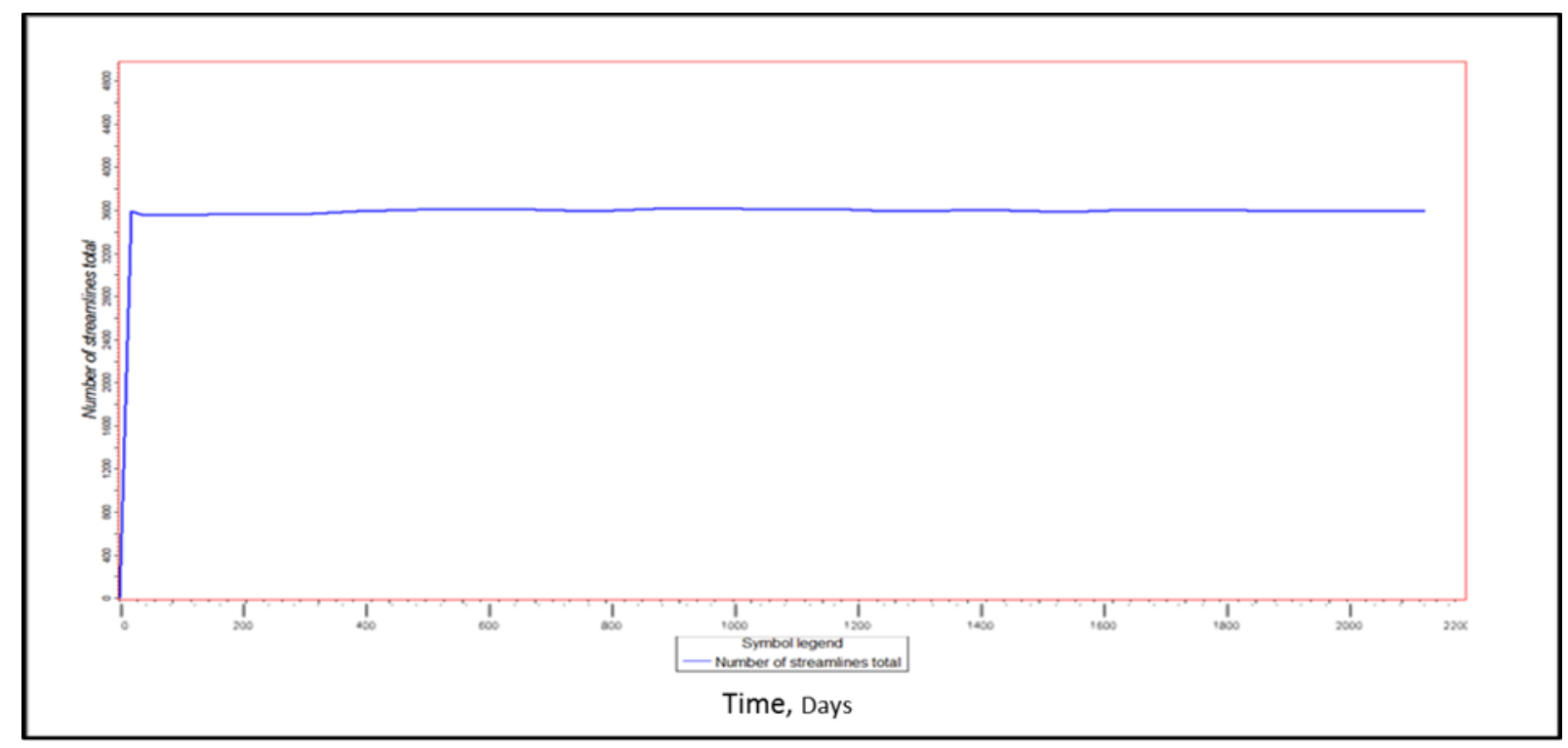

Figure 5-12: Total Number of Streamlines Generated for Water Injection Model

\subsubsection{Water Alternating Gas (WAG) Model}

The main objective of the injection process is to squeeze more oil out of the reservoirs as it is well known that there is remaining (residual) oil in the flooded rock. In this process, a gas slug is followed by a water slug or combined water/gas injection can be done also, and it is recommended to improve the gas injection sweep efficiency by using water to control the mobility and to stabilise the front. Water injection alone tends to sweep the lower parts of a reservoir, while gas injection alone sweeps more of the upper parts of a reservoir owing to the gravity forces. Three-phase gas, oil and water flow is favoured at displacing residual oil in the pore system than two-phase flow. The model permits the use of primary drainage, imbibition and secondary drainage data for both gas-oil and oil-water systems. Figure 5-13 represents the schematic diagram of the WAG Process. 


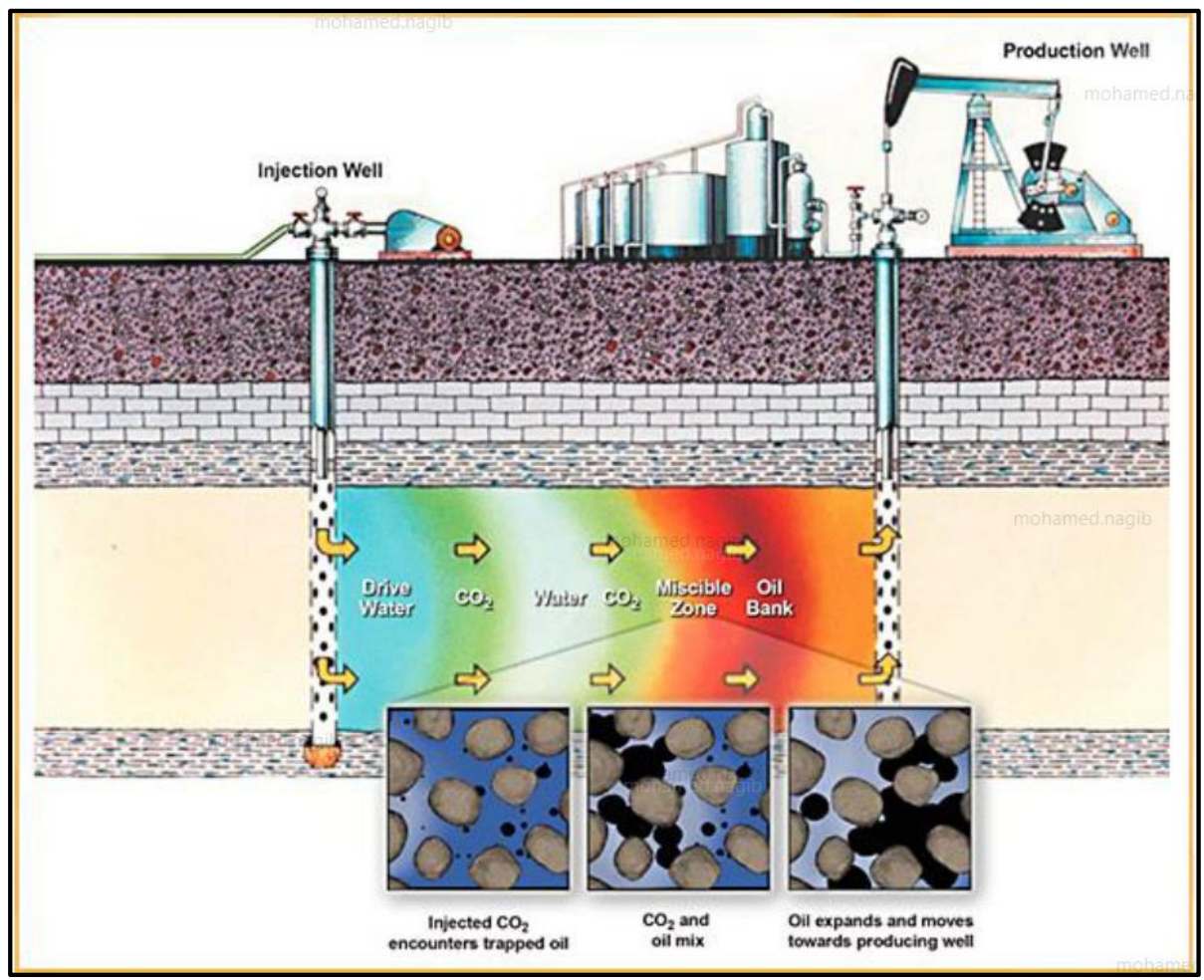

Figure 5-13: Schematic representation of WAG injection Process (U.S Department of Energy, 2013)

In this case, the model used in the previous case for Water Flood was modified to be a Water Alternate Gas (WAG) model. This alternate both water and gas injection into the reservoir to achieve increased recovery. The following grid view shows the oil saturation grid in both FD and SL simulation models to show the progression and the direction of sweep towards the producers. Figure 5-14 Shows Oil saturation grid for WAG FD and SL Models. As can be seen in the graph, the upper two graphs show the FD simulation and the bottom two graphs show the SL simulation model. Good agreement is shown for the oil saturation grid both at the first- and last-time steps. 


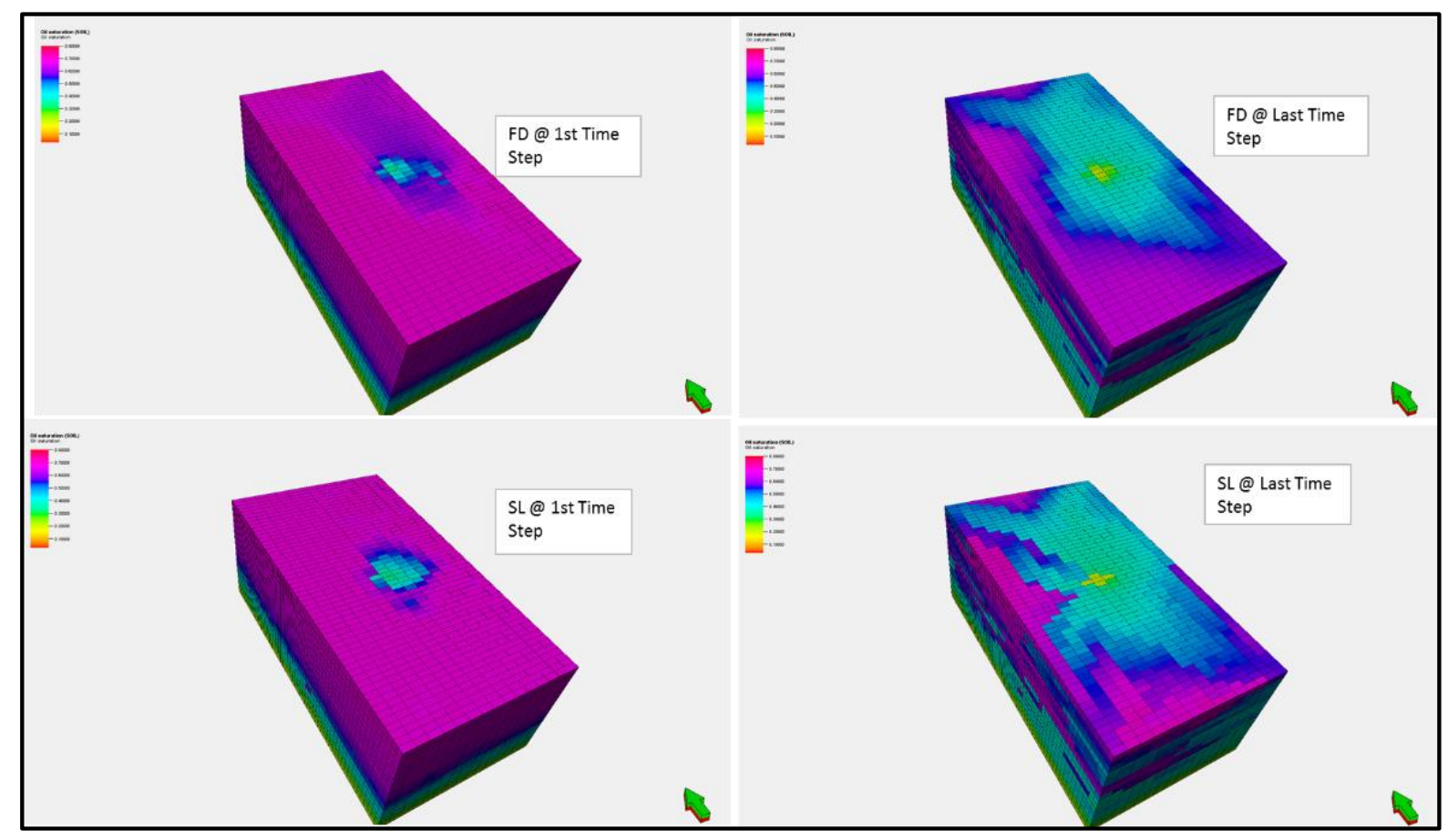

Figure 5-14: Oil Saturation Grid for WAG Models at Different Time Steps

\subsubsection{WAG Model Performance}

With the WAG injection model, predictions of oil production performance are presented in Figure 5-15, and total oil production in Figure 5-16. A total oil production of 2.4 million barrels over a 6-year period was achieved. Water was initially injected for a duration of 90 days to understand the effect of displacement of oil in the reservoir, and a cumulative of over 600,000 barrels was achieved. Gas was later injected into the reservoir for the same number of days. Gas injection yielded a cumulative of over 158,000 barrels. Both injections were alternated subsequently for the next six years with injection periods of 90 days each. A detailed analysis and comparison of both FD and SL simulation models are presented in this section. SL technology has already been proved and used now for decades in modelling and optimisation of water flood projects. So, less relative errors and differences were expected to be seen from these results. In the following section, detailed 2D graphs representing a detailed comparison of predictions of both FD and SL simulation models are presented. There are small mismatches in the middle of the simulation time. 
This could be due to differences in the time steps calculated by the FD simulation in comparison to the SL simulation. Figure 5-15 represents the oil production rate in both cases of FD and SL simulation.

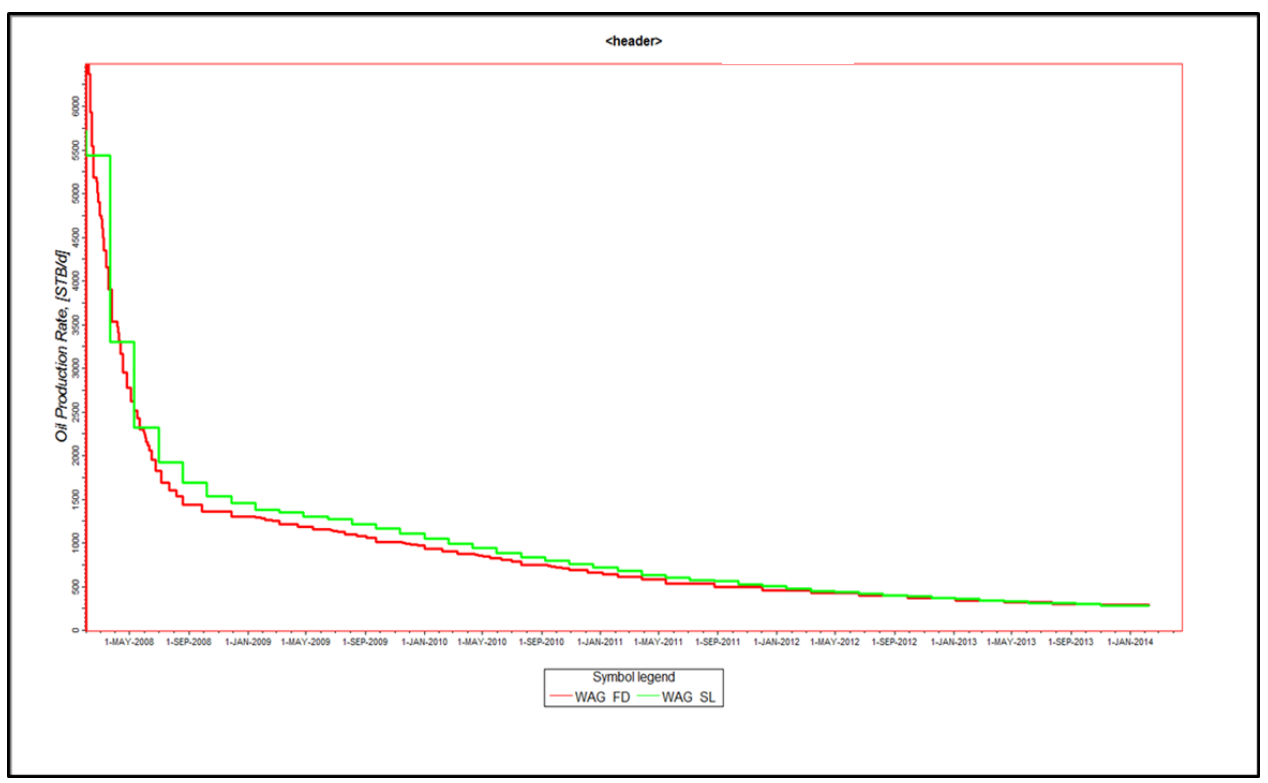

Figure 5-15: Comparison of Oil Production Rates of FD and SL WAG Models

Figure 5-16 shows a comparison of the total oil production of both FD and SL simulation models. As a result of the differences in production rates, an incremental difference of $8 \%$ is observed.

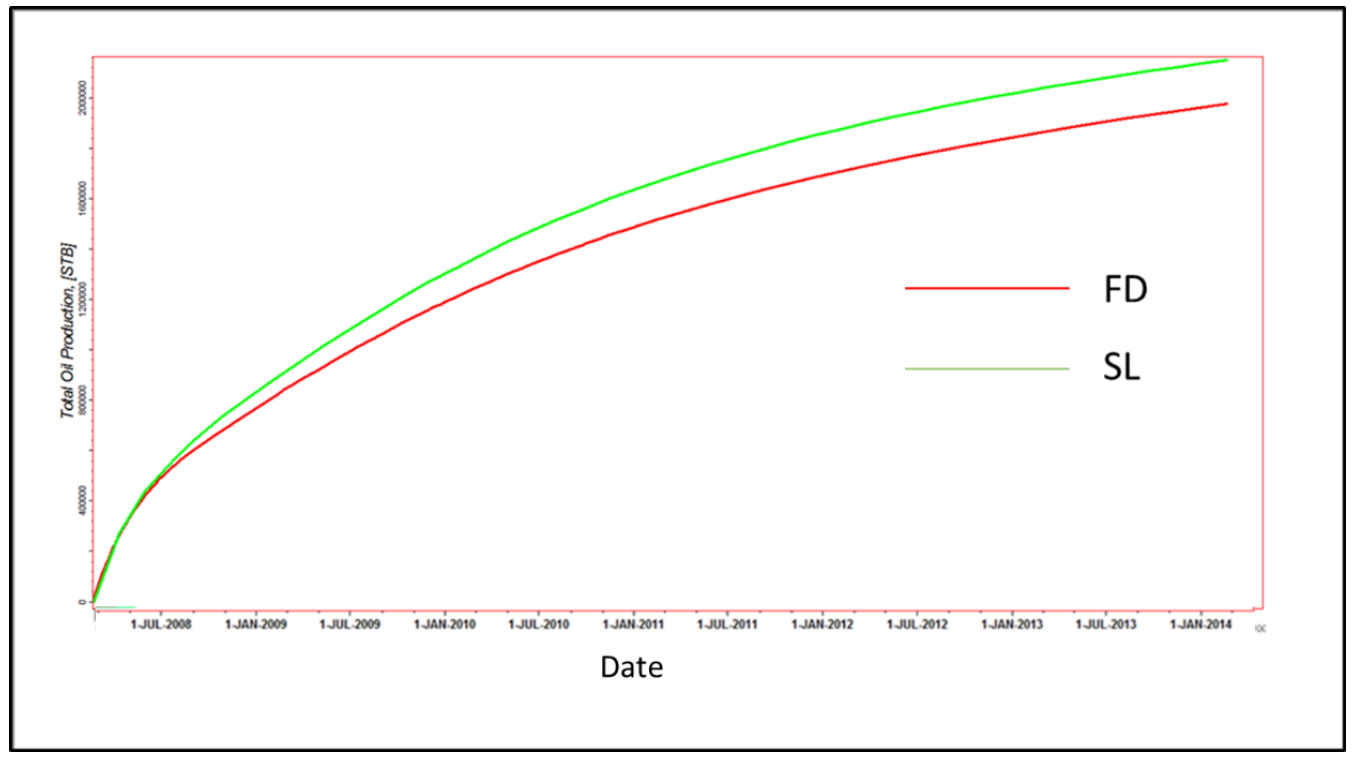

Figure 5-16: Comparison of total Oil Production of FD and SL WAG Models 
With regards to the water production profiles comparisons, Figure 5-17 shows the water production profiles for the water injection model for both SL and FD Models. There is a good match at later times. However, there are large differences in the initial transient which settle down to a maximum of $9 \%$ difference at intermediate times. This is probably due to the use of different time steps in the SL and FD WAG models.

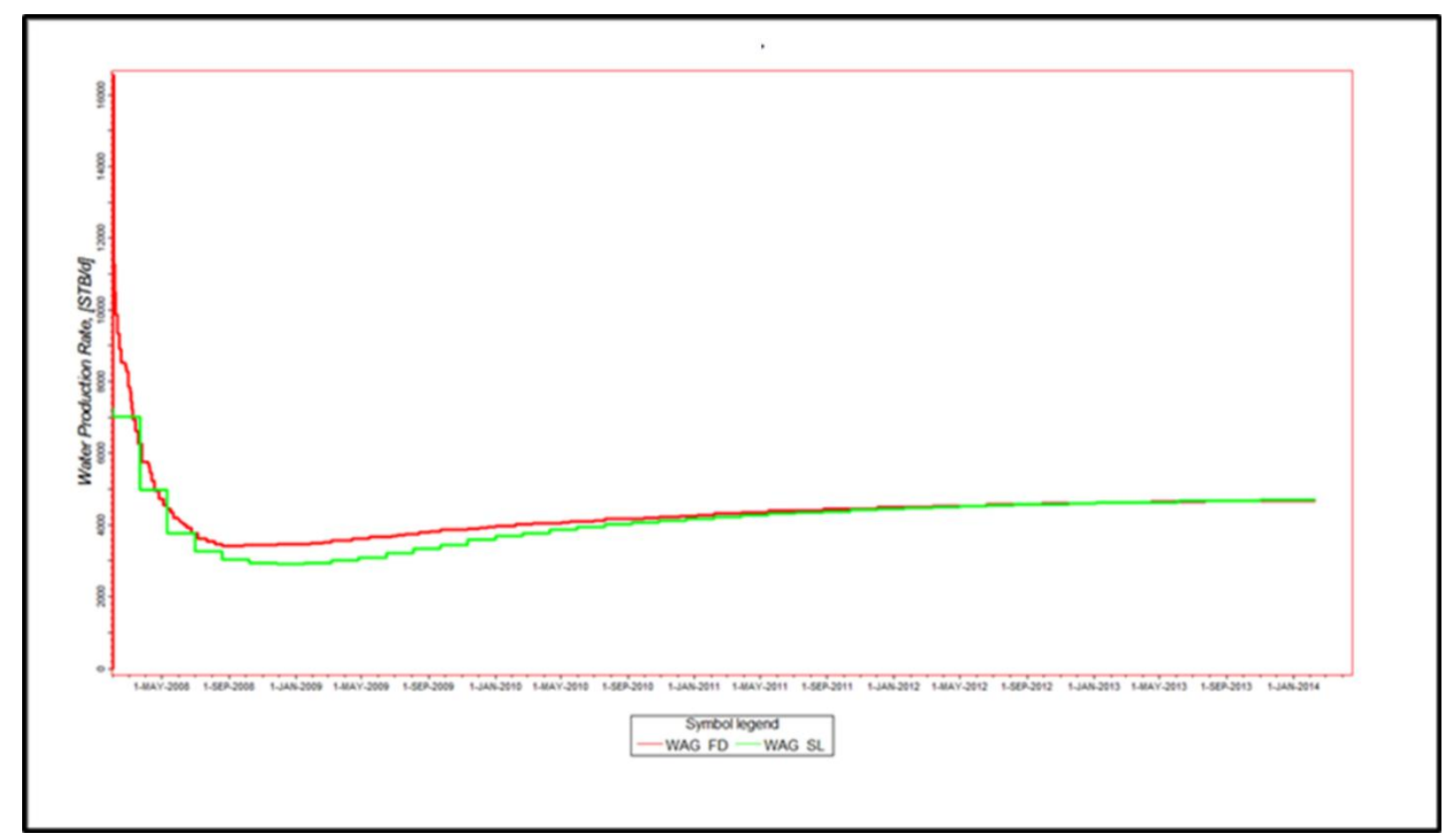

Figure 5-17: Comparison of Water Production Rates of FD and SL WAG Models

Water cut performance for both models are presented in Figure 5-18. As can be observed from the graph, agreement is good at later times, with a difference of around $9 \%$ occurring at earlier times 


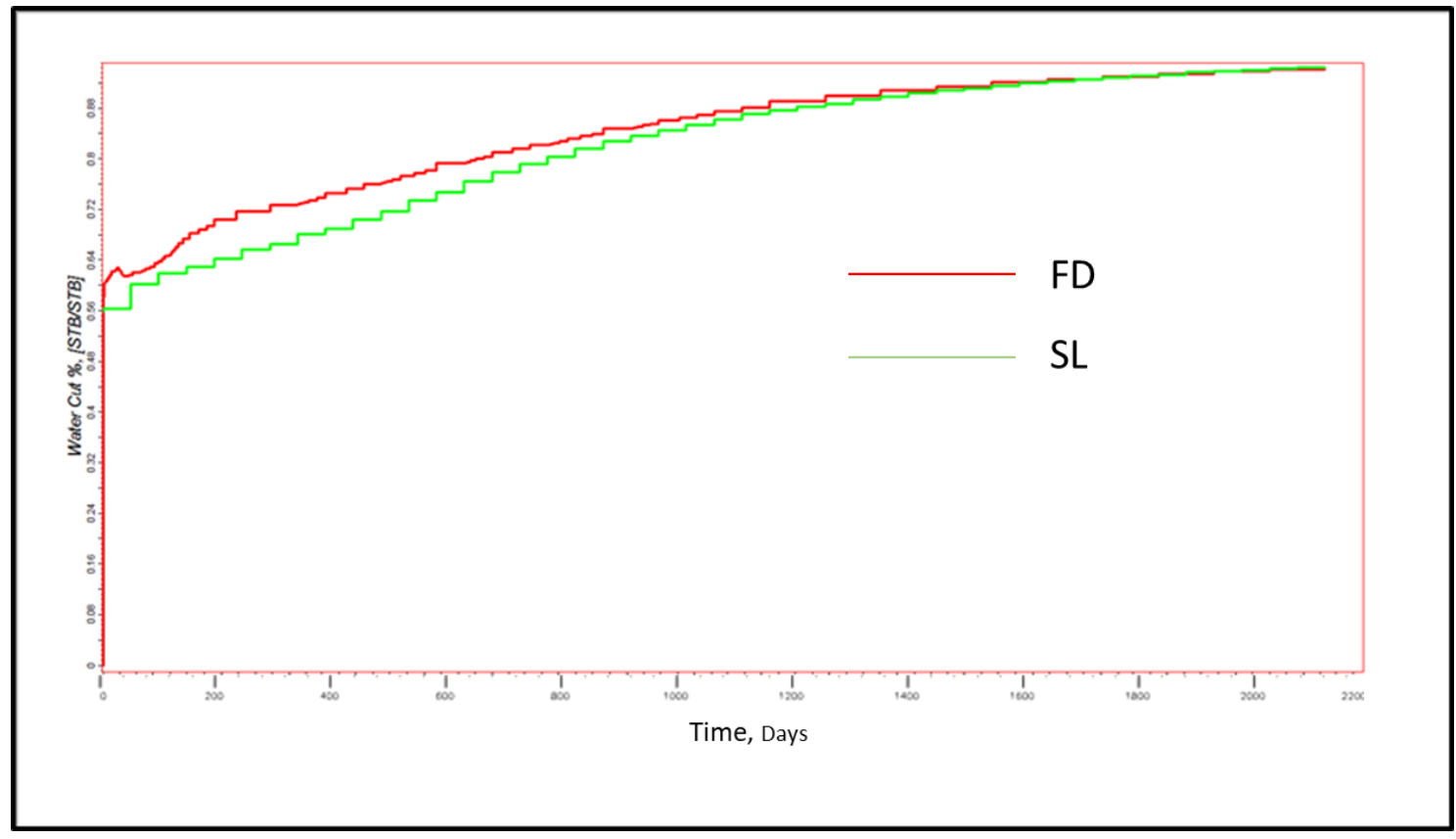

Figure 5-18: Water Cut Performance for Water flooding Model

Figure 5-19 represents the pressure performance for the WAG case. As indicated from the graph, the initial reservoir was +/-6000psi in both SL and FD simulation models. Once production and injection started, a decline in reservoir pressure was noticed, and this continued until the impact of the response of injecting both water and gas inside the reservoir was noticed. After this, the reservoir pressure became more sustainable with minimum decline.

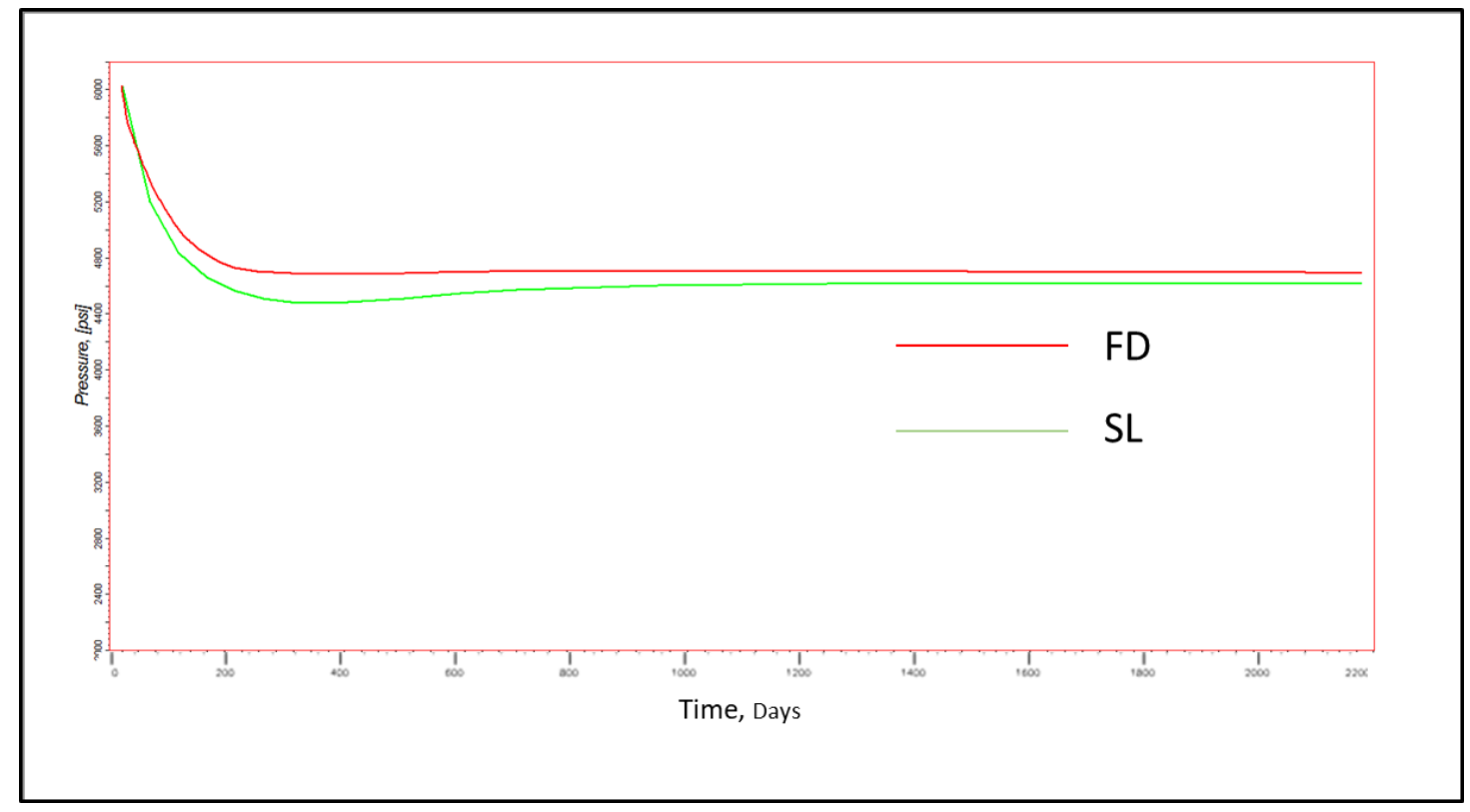

Figure 5-19: Comparison of Pressure Performance of FD and SL WAG Models 
In order to cover all the controlling parameters/factors affecting the simulation performances of both FD and SL simulation models, in this section detailed 2D plots comparisons of the following vectors are be presented:

- Total CPU used for FD vs. SL

- Detailed distribution of CPU required and used for each solver on the SL simulation model

- Detailed numbers of Newton iterations used by FD vs. SL

- Detailed numbers of Linear iterations used by FD vs. SL

- Total number of streamlines used in the SL model.

Figure 5-20 represents the total CPU required to complete the simulation of both FD and SL models. As indicated on the graph, almost four times the amount of CPU was consumed for the FD simulation model in comparison to the SL simulation model.

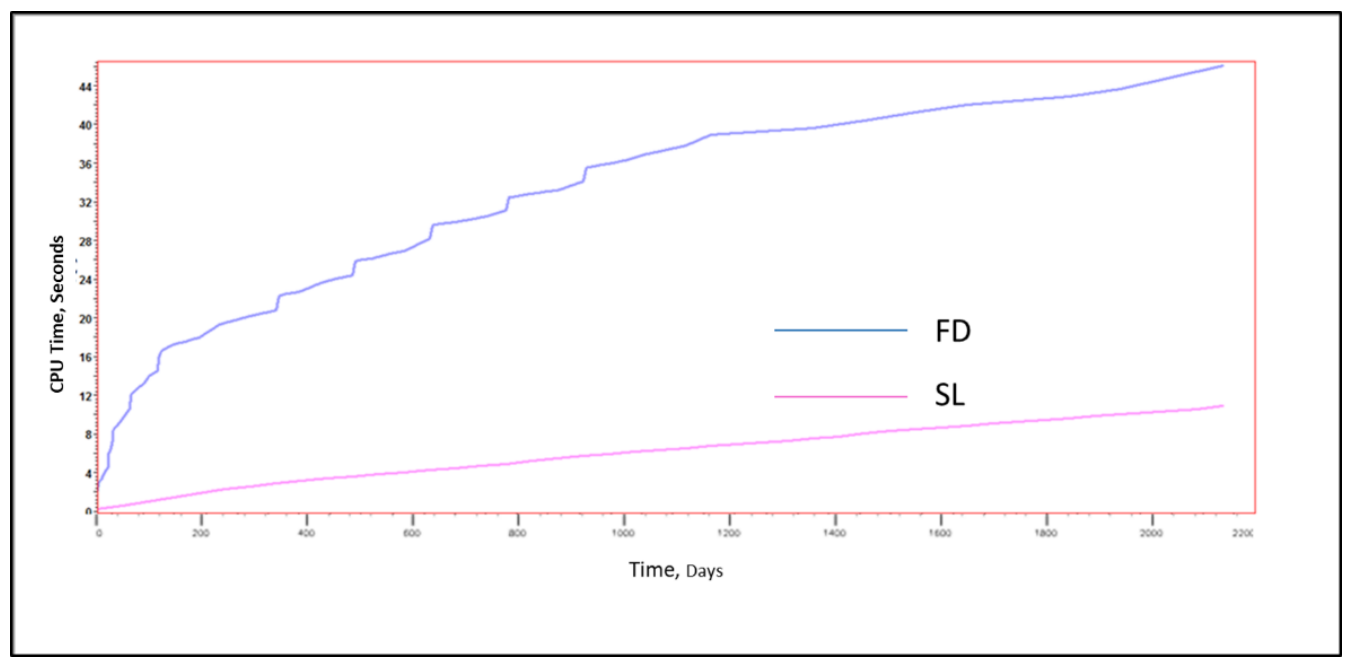

Figure 5-20: Total CPU Comparisons for FD and SL WAG Models

Figure 5-21 represents the detailed CPU time distribution in order to complete the simulation run. As can be seen from the graph, the highest amount of CPU time is taken by the saturation solver, followed by the pressure solver. Assembling the matrix as both takes the same amount of CPU time. Both initialization and velocity solvers employ the lowest amount of CPU time. 


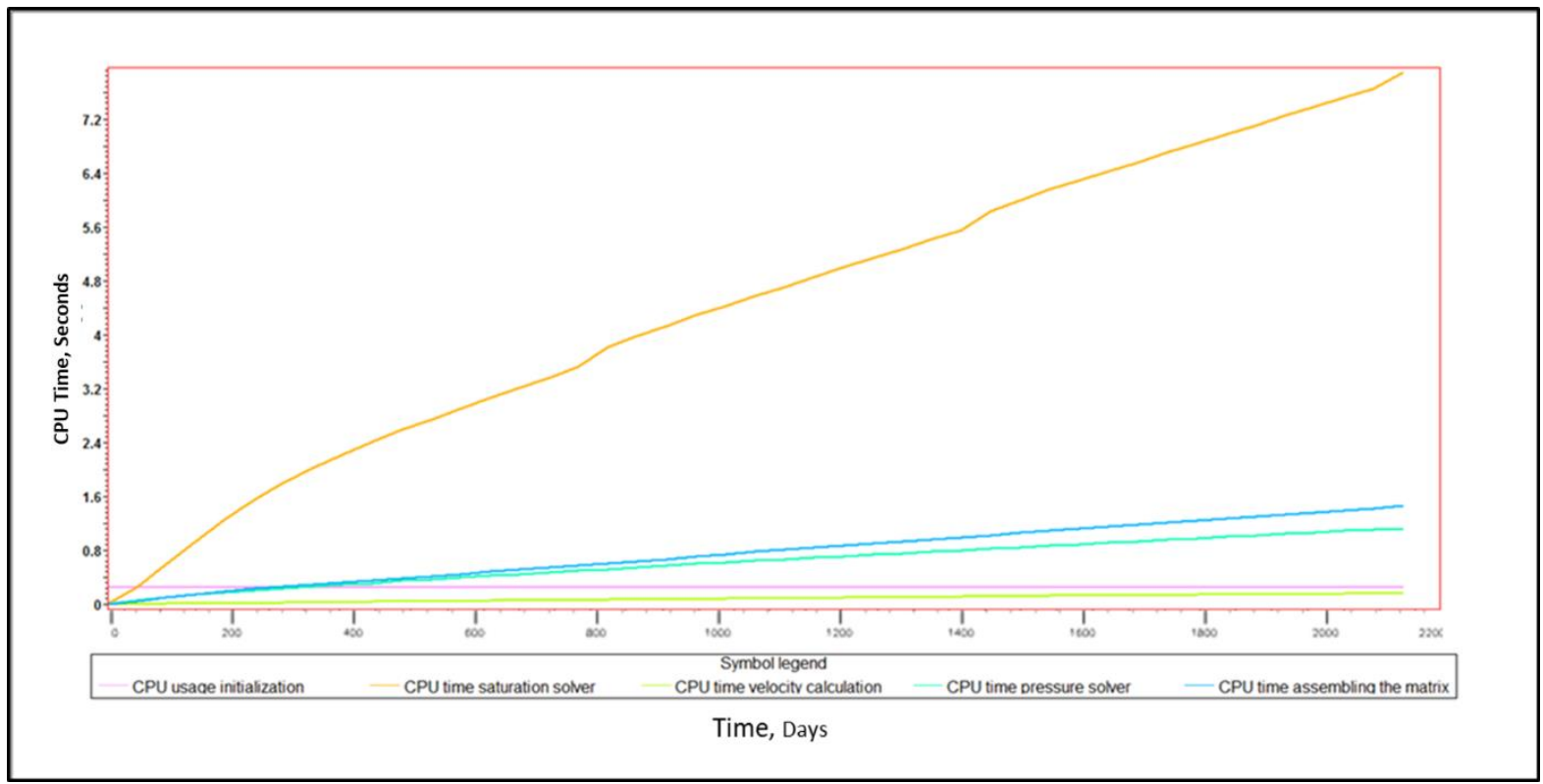

Figure 5-21: CPU Time Required for Different Solvers used for SL Calculations

Figure 5-22 represents a comparison of the number of Newton iterations used for FD vs. SL simulation models. As can be seen, the SL simulation model used a lower number of Newton iterations and is more stable in comparison to the FD simulation model. The same observation can be concluded from Figure 5-23 for the linear iteration of FD vs. SL simulation model as well. The total number of streamlines generated for the WAG SL Model is presented in Figure 5-24. The averaged total number of streamlines generated for this model is equal to 200 .

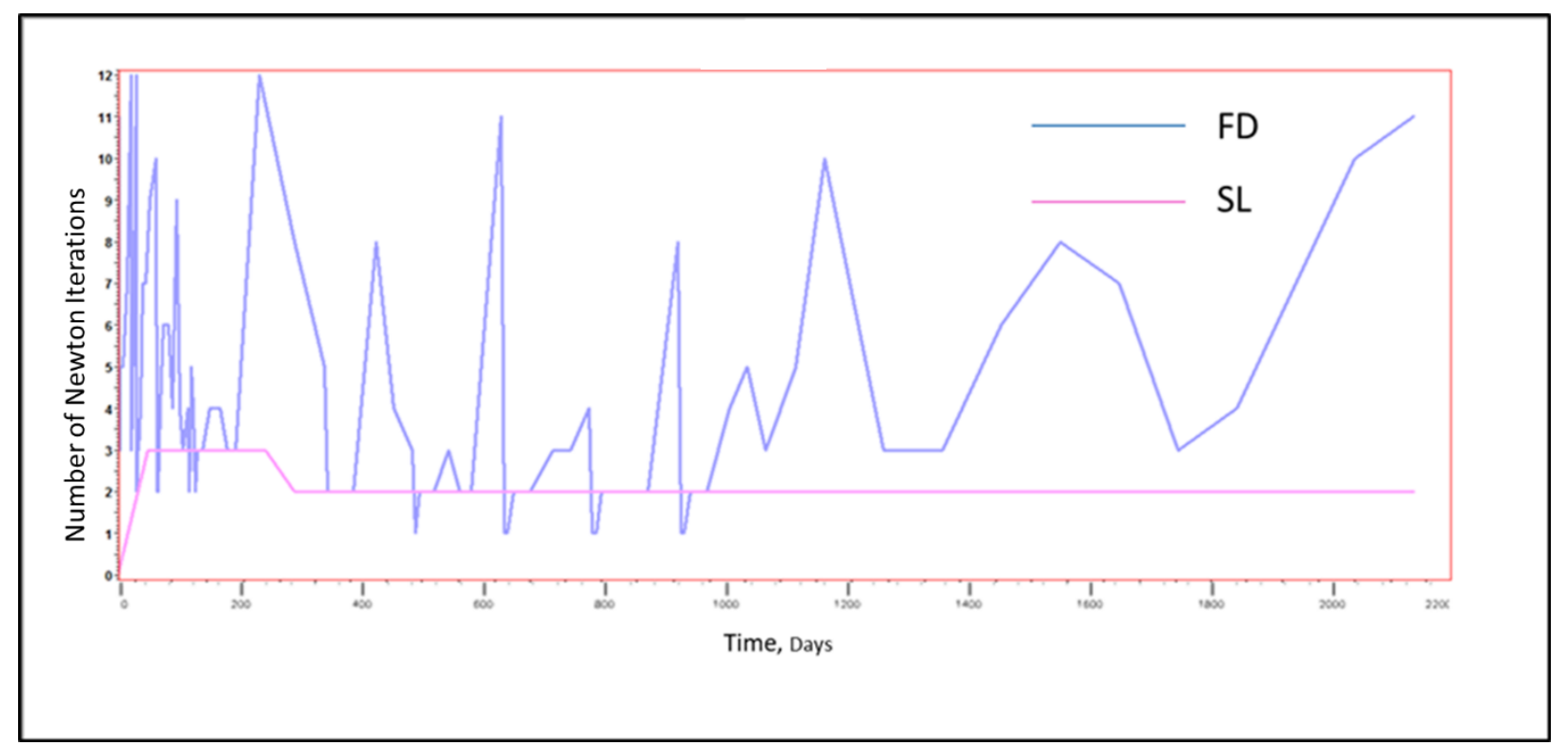

Figure 5-22: Comparisons of Number of Newton Iterations Calculated for FD and SL Models 


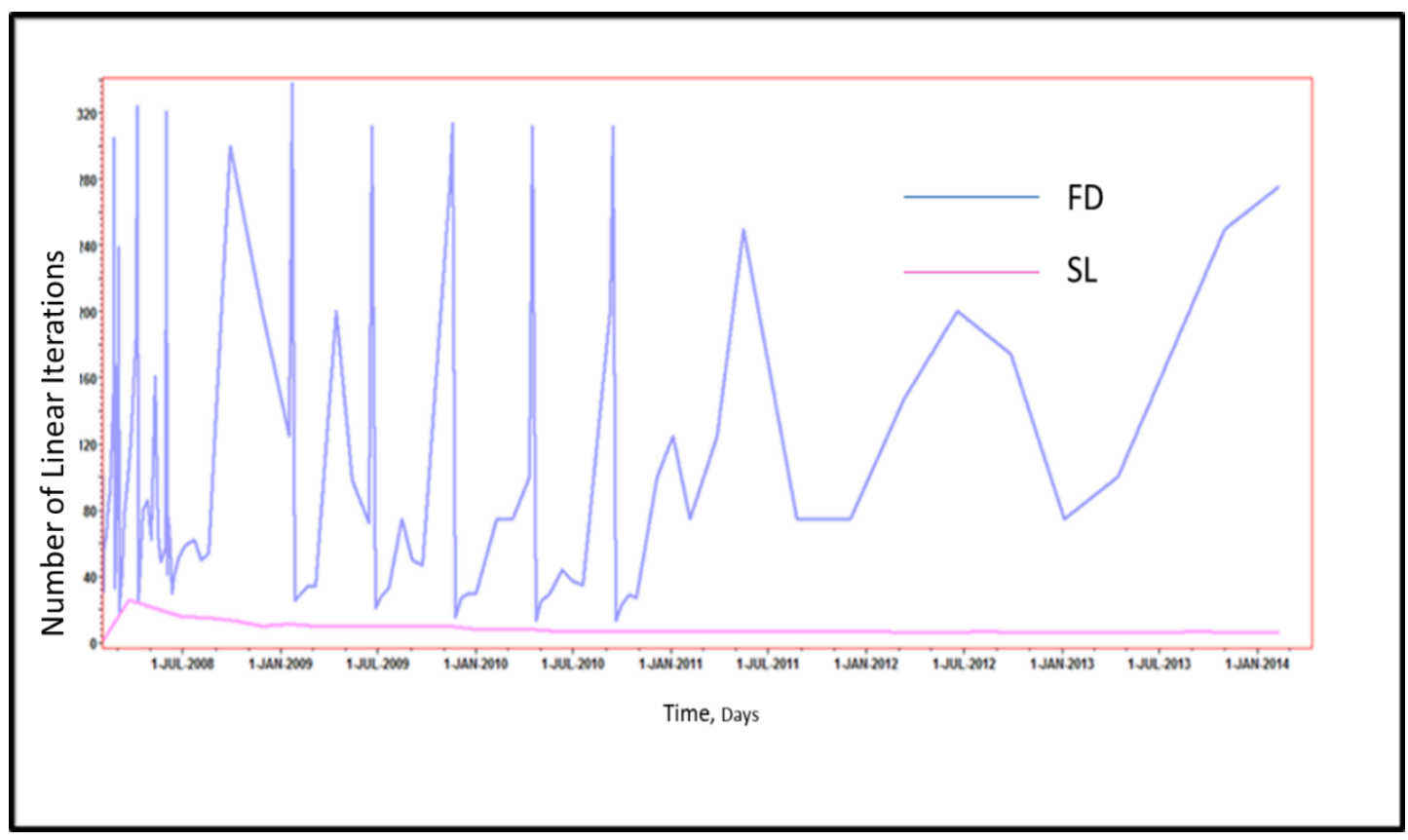

Figure 5-23: Comparisons of Number of Linear Iterations Calculated for FD and SL Models

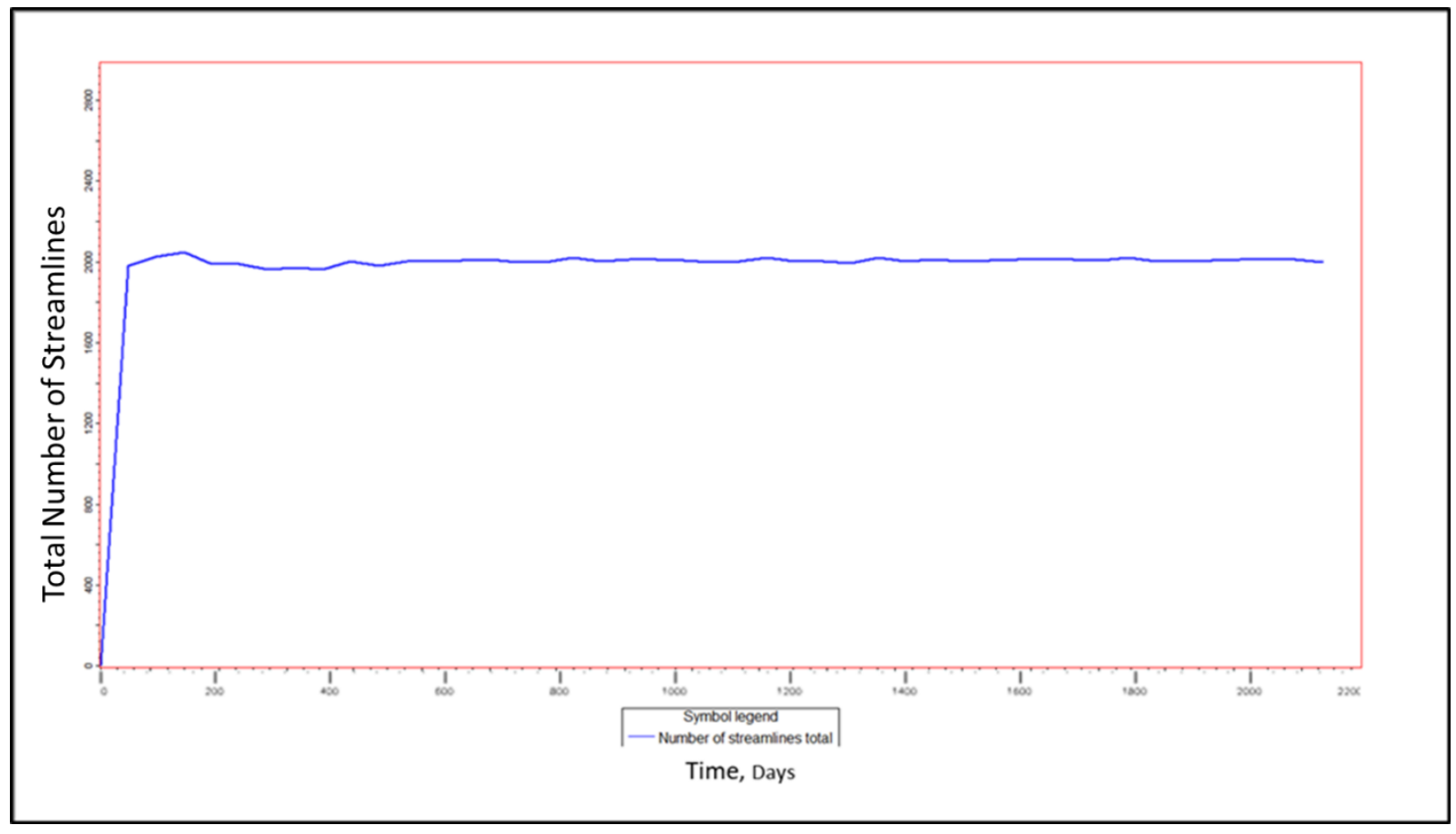

Figure 5-24: Total Number of Streamlines Generated for the WAG SL Model 


\subsubsection{Gas Injection Model}

The initial model set up was the same as the original SPE10 water injection scheme, except the gas is to be injected instead of water, but all other model parameters are the same as the water flood model; completion intervals, well placements, the PVT data and relative permeability curves used are the same. A three-dimensional rectangular reservoir model is investigated on the SPE 10 model and the grid dimensions are $17 * 55 * 15$ grid blocks.

\subsubsection{Dead Oil and Live Oil/Dissolved Gas}

The Original SPE Tenth Comparative Solution Model was developed with Dead Oil PVT structure, considering three phase fluid structure for gas injection and water alternating gas (WAG) led to further improvement of the PVT structure to Live Oil (i.e. Dissolved gas with water and oil). The improvement of the PVT Live Oil structure led to a perfect case study for comparison during the analysis of the result.

Dead oil is the oil usually at sufficiently low pressure that it does not contain dissolved gas, or a relatively thick oil, or residue that has lost its volatile components. Live oil is oil containing dissolved gas in solution that may be evolved from solution at surface conditions. Live oil should be handled and pumped under closely controlled conditions to avoid the risk of explosion or fire. Figure 5-25 shows oil production performance for both the SL and FD models and as per the plot, the streamline model runs only for six months of the production before the model stops running. A subsequent analysis showed that there is a material balance problem in the model, when gas can no longer be injected into the reservoir with the properties of the dead oil initially characterised as in the water injection case. At sufficiently low pressure, the oil does not contain dissolved gas or a relatively thick oil or residue that has lost its volatile components. The dead oil PVT and relative permeability responses no longer allow the injection of the gas into the reservoir and hence the oil can no longer be displaced. Many trials were then made in order to improve the flow dynamics within the model using tuning factors and other available simulation controls in the simulator, but no major improvements were achieved. The conclusion was that dead oil cannot be used in streamline simulation for the case of gas displacement processes. Figure 5-26 and Table 5-1 show respectively oil water relative permeability data and gas oil relative permeability data sets used for the initial runs. 


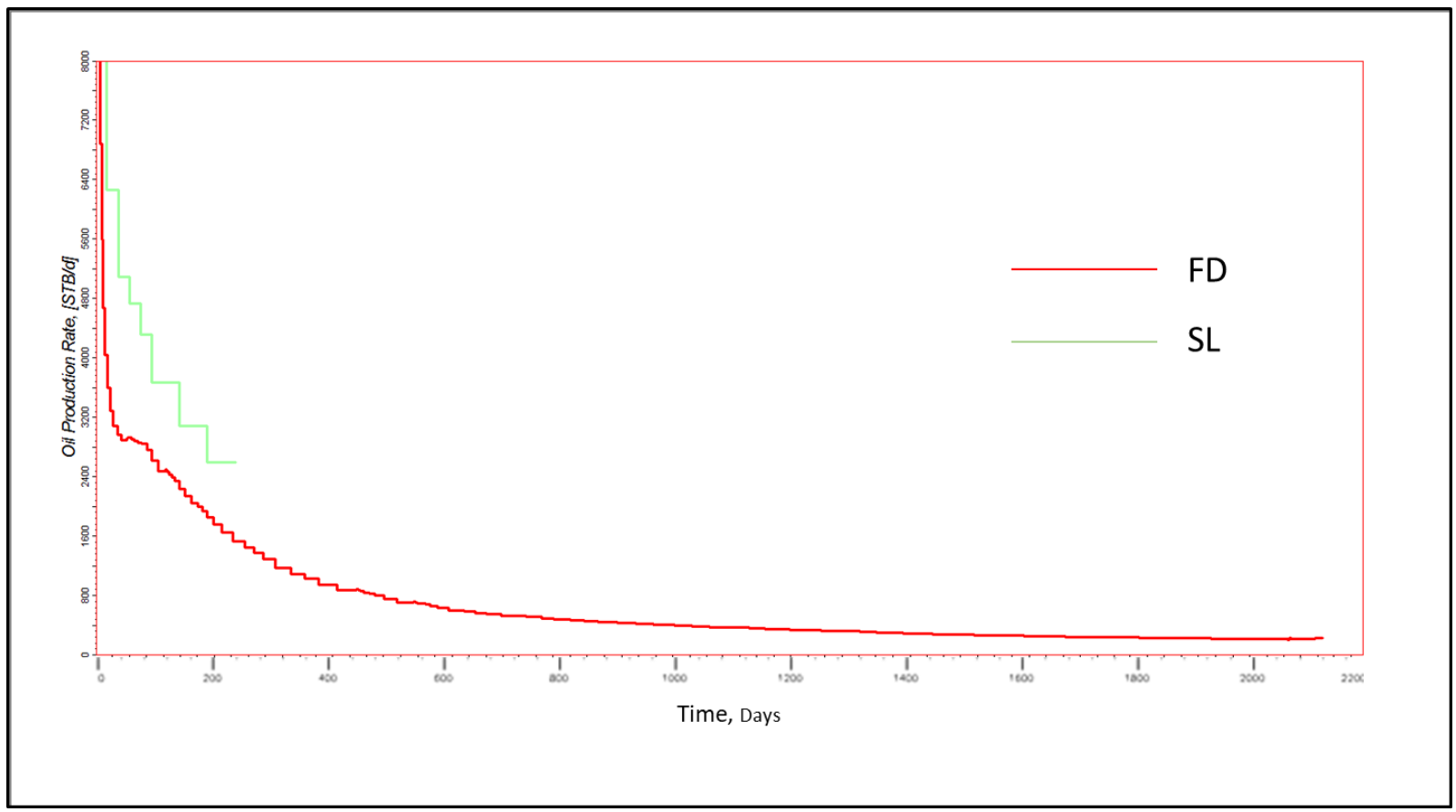

Figure 5-25: Comparison of Oil Production Rates of FD and SL Dead Oil Gas Injection Models

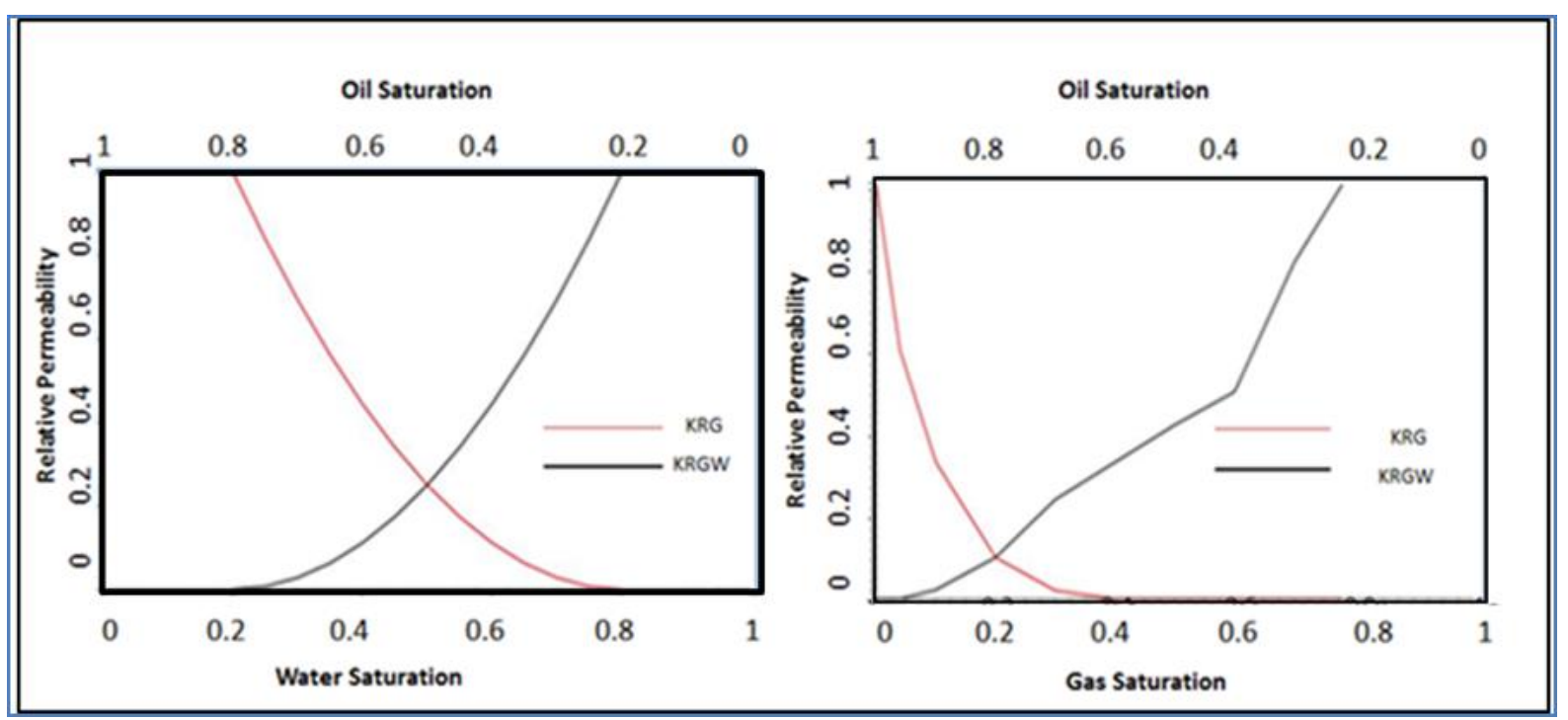

Figure 5-26: Oil and Gas Relative Permeability Data Set used for the Initial Run (Dead Oil). 
Table 5-1 : Oil and Gas Relative Permeability Data (Dead Oil).

\begin{tabular}{|c|c|c|c|c|c|}
\hline \multicolumn{3}{|c|}{ Water - Oil Relative Permeability } & \multicolumn{3}{|c|}{ Gas to Oil Relative Permeability } \\
\hline $\begin{array}{l}\text { Water } \\
\text { Saturation }\end{array}$ & $\begin{array}{l}\text { Water Relative } \\
\text { Permeability }\end{array}$ & $\begin{array}{l}\text { Capillary Pressure } \\
\text { to Water }\end{array}$ & $\begin{array}{l}\text { Gas } \\
\text { Saturation }\end{array}$ & $\begin{array}{l}\text { Gas Relative } \\
\text { Permeability }\end{array}$ & $\begin{array}{l}\text { Capillary Pressure } \\
\text { to Gas }\end{array}$ \\
\hline 0.0 & 0.0 & 0.00 & 0.20 & 0.000 & 1.00 \\
\hline 0.04 & 0.0 & 0.20 & 0.25 & 0.007 & 0.84 \\
\hline 0.10 & 0.02 & 0.50 & 0.30 & 0.028 & 0.69 \\
\hline 0.20 & 0.10 & 1.00 & 0.35 & 0.062 & 0.56 \\
\hline 0.30 & 0.24 & 1.50 & 0.4 & 0.111 & 0.44 \\
\hline 0.40 & 0.34 & 2.00 & 0.45 & 0.17 & 0.34 \\
\hline 0.50 & 0.42 & 2.50 & 0.5 & 0.25 & 0.25 \\
\hline 0.60 & 0.50 & 3.00 & 0.56 & 0.34 & 0.17 \\
\hline 0.70 & 0.81 & 3.50 & 0.60 & 0.44 & 0.11 \\
\hline 0.78 & 1.0 & 3.90 & 0.66 & 0.56 & 0.06 \\
\hline & & & 0.70 & 0.69 & 0.03 \\
\hline & & & 0.75 & 0.84 & 0.07 \\
\hline & & & 0.80 & 1 & 0.00 \\
\hline
\end{tabular}

\subsubsection{Conclusion}

In this chapter, three cases were presented for the $10^{\text {th }}$ SPE model. One was for Water Injection, which was then converted into a Water Alternate Gas Scheme, and the third one was the base case Gas Injection model.

The latter used dead oil PVT and relative permeability data and the preliminary results showed that streamline simulation cannot be run in the case of dead oil as per the data presented earlier in case of Gas Injection. However, both water injection and WAG cases are in good agreement when compared with FD simulation results. 


\section{Chapter 6}

\section{Baseline Gas Injection Model}

\subsection{Initial model set up}

In the previous chapter, the SPE 10 model was initially used for water flooding streamline techniques, and then this five-spot water injection pattern model was converted to a gas injection model. The objective of this research is to evaluate the applicability of streamline simulation with gas displacement processes. The initial model set up was the same as the original SPE10 water injection scheme except gas is to be injected instead of water, but all other model parameters are similar to the water flood model; completion intervals, well placements, the PVT data and relative permeability curves used were the same. On SPE 10, a three-dimensional rectangular reservoir model was considered, with grid dimensions of $(17 * 55 * 15$ grid blocks).

Horizontal permeability varies six orders of magnitude and vertical permeability varies ten orders of magnitude. Porosity is strongly correlated with permeability and initially the reservoir is filled with $70 \%$ oil saturation and 30\% water saturation. Figure 6-1 shows the permeability distribution and well configurations for the Tarbert formation used on the Gas Injection model.

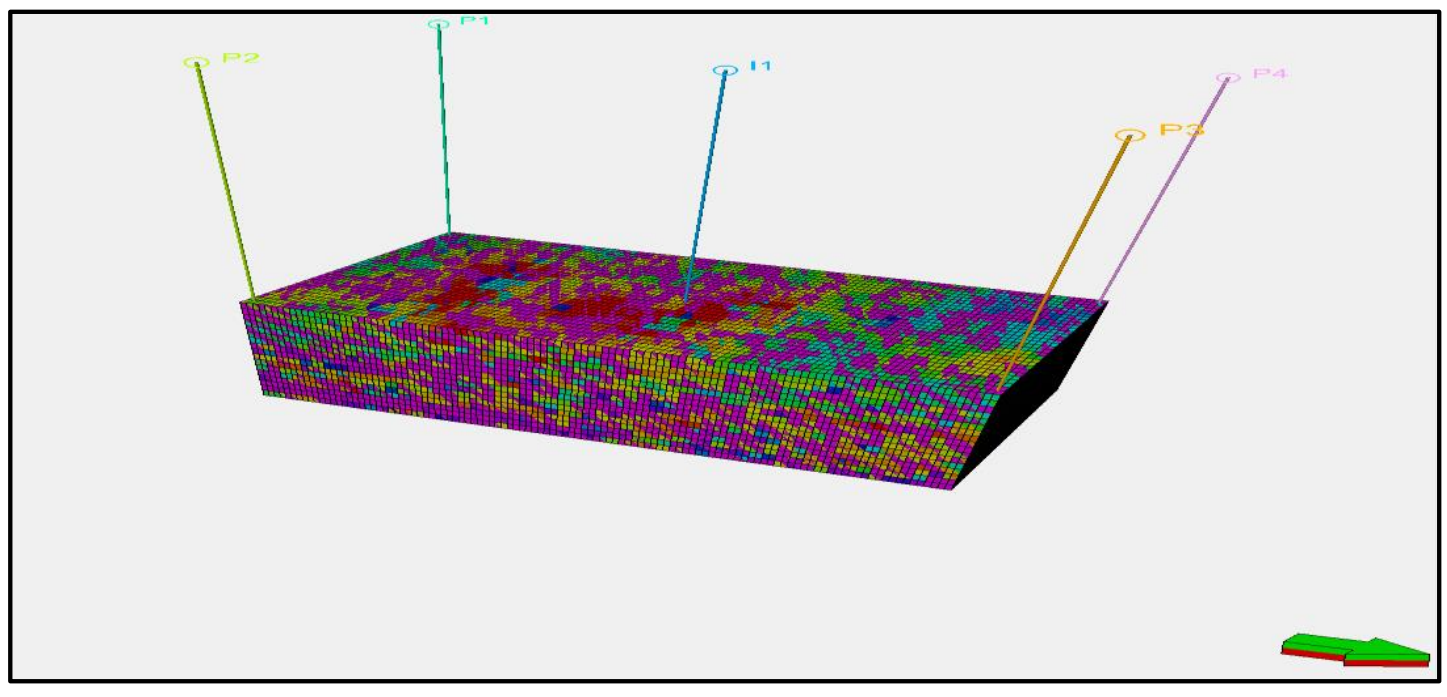

Figure 6-1: 3D Permeability Distribution and Well Configurations for the Tarbert formations. 
As per the initial results presented in the last part of the previous chapter, the SL model runs for only six months of production before the model stops running. A subsequent analysis showed that there is a material balance problem in the model, when gas can no longer be injected into the reservoir with the properties of the dead oil initially characterised in the water injection case. This occurs for oil at sufficiently low pressure that it contains no dissolved gas or a relatively thick oil or residue that has lost its volatile components. The dead oil PVT and relative permeability responses no longer allow the injection of the gas into the reservoir and hence the oil can no longer be displaced. Many trials were then made in order to improve the flow dynamics within the model using tuning factors and other available simulation controls in the simulator, but no major improvements were achieved. The conclusion was that dead oil cannot be used in streamline simulation for the case of gas displacement processes.

Live oil was then used and indeed the flow dynamics in the reservoir improved considerably, so that the streamline model could be run for the same period as the FD simulator. Table 6-1 below illustrates both oil and gas relative permeability alternative data set used for the initial runs and Figure 6-2 below shows the same data in a graphical display.

Table 6-1: Alternative Oil and Gas Relative Permeability Data Set used for the Base Case (Live Oil).

\begin{tabular}{|c|c|c|c|c|c|}
\hline \multicolumn{2}{|c|}{ Water - Oil Relative Permeability } & \multicolumn{3}{c|}{ Gas to Oil Relative Permeability } \\
\hline SW & KRW & PCW & SG & KRg & Pcg \\
\hline 0.22 & 0 & 7.0 & 0.00 & 0.00 & 0.0 \\
\hline 0.30 & 0.07 & 4.0 & 0.04 & 0.00 & 0.2 \\
\hline 0.40 & 0.15 & 3.0 & 0.10 & 0.02 & 0.5 \\
\hline 0.50 & 0.24 & 2.5 & 0.20 & 0.10 & 1.0 \\
\hline 0.60 & 0.33 & 2.0 & 0.30 & 0.24 & 1.5 \\
\hline 0.80 & 0.66 & 1.0 & 0.40 & 0.34 & 2.0 \\
\hline 0.90 & 0.83 & 0.5 & 0.50 & 0.42 & 2.5 \\
\hline 1.00 & 1.00 & 0.0 & 0.60 & 0.50 & 3.0 \\
\hline & & & 0.78 & 0.81 & 3.5 \\
\hline
\end{tabular}




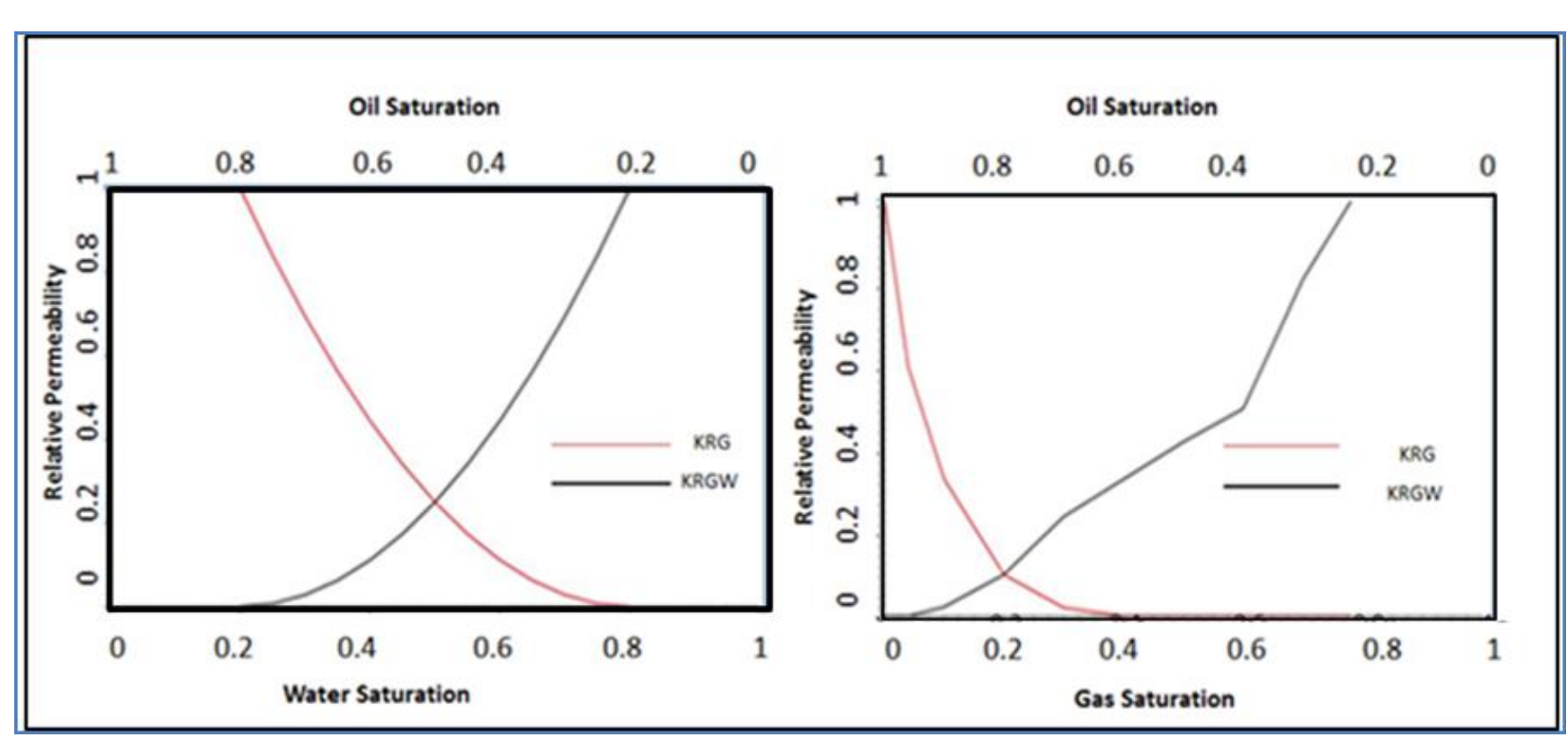

Figure 6-2: Alternative Oil and Gas Relative Permeability data set used (Live Oil)

As a result of the improvement of the flow dynamics inside the model due to live oil, the streamline model now is able to run and cover the full period targeted. The reason for this is the live oil is connected with how the gas is able to flow inside the reservoir. Hence, the sweep and oil recovery are improved.

Figure 6-3 below shows the comparison between the two simulators after the adjustment of the relative permeability curves used in the live oil model.

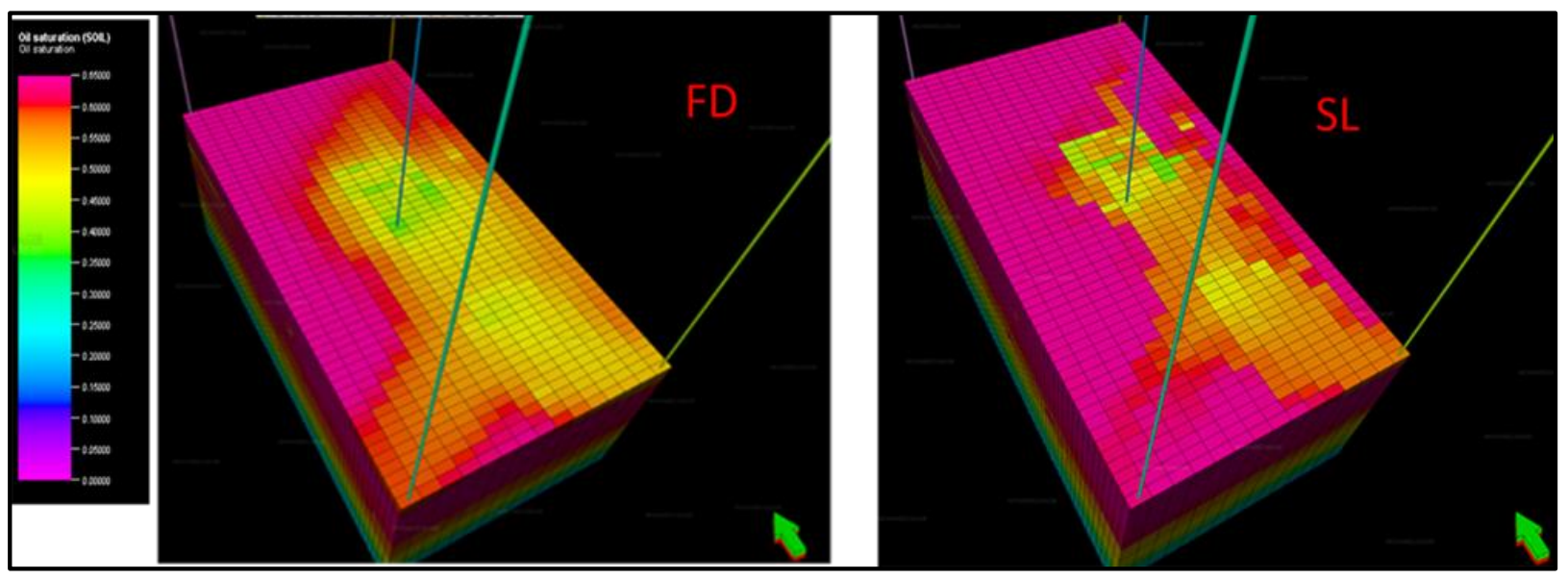

Figure 6-3: Oil Saturation Displayed grid for both FD and SL Gas Injection Models 
The plot above shows the oil saturation distribution for the FD model versus the SL simulation model. As can be seen, the streamlines reveal gas displaced from injector to producers. As reported in the previous chapter, and as expected, there were still mismatches in terms of oil rate, cumulative oil production and water production. Figure 6-4 below shows oil production rates for both FD and SL models.

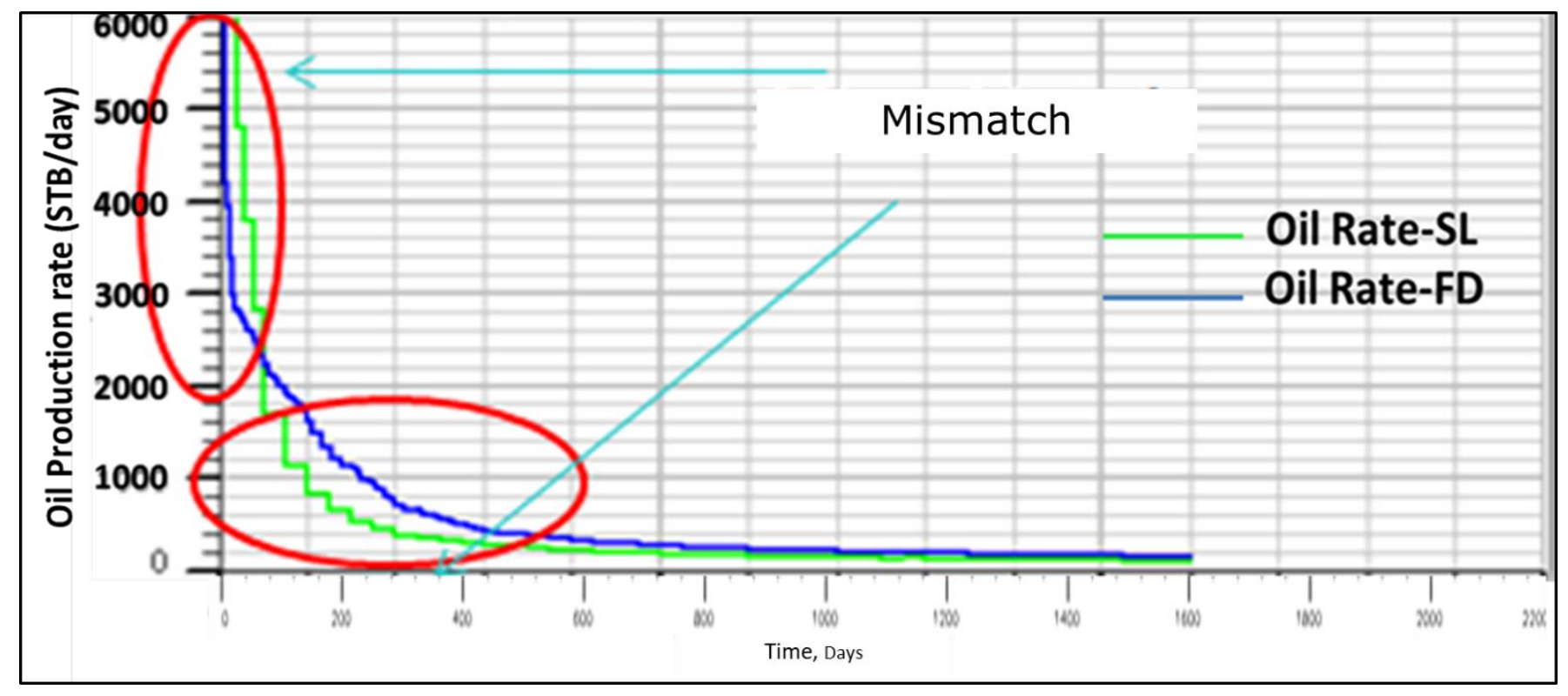

Figure 6-4: Oil production profile for both FD and SL.

There is a mismatch in the oil rate right from the start date; the streamline simulation shows similar maximum scaling of the oil rate. However, there is enhanced oil production (the area under the rate graph) compared to the finite difference model in the early period of simulation time for approximately the first 100 days. After 100 days, the SL streamline oil rate decreases more quickly in this 'middle period'. At around 1000 days, the rates begin to converge again in a classic asymptotic decline pattern. In the following section, we investigate and explain how the flow regimes inside the models are different, and what is required to improve the flow in the streamline model in order to minimise the differences between FD and SL.

The focus now is on gas displacement process matching and the fine-tuning of the streamline simulation model, quantifying, analysing the differences and trying to minimise them to be within the applicable limits such as in the water injection streamline case. This can be done in two ways. Firstly, we analyse how the SL simulation works and consider the implications of the gas displacement process on the fluid movement into the reservoir. Secondly, we analyse and adjust the parameters and controlling factors in the simulation in order to improve the flow and minimise the differences, in order that an acceptable match can be reached. 
As per the results presented in Figure 6-4 previously, the final acceptable degree of match has not yet been obtained, and according to the analysis of the results, it was concluded that SL time steps calculations and reporting is still not capable of matching the FD results, as FD is able to report and handle any required time steps. To control this and to improve the SL calculation, additional tuning improvements and fine controlling of the time steps is required to reach an acceptable level of match. Therefore, in order to enhance the match, additional control parameters were introduced from FD to SL and sensitivities were performed. For example, the initial rates and large numbers of time steps help to improve and minimise the error differences between both finite difference and streamline simulation models. A series of iterations and sensitivities have been performed and discussed below.

\subsection{Streamline sensitivities to improve the flow inside the model}

If the main motivation for using streamlines to solve for the fluid flow is computational speed, it is also an attractive feature of streamline modelling that streamlines form a 'natural' grid for the transport equations. Over this natural grid, a choice of numerical methods could be applied. Modern streamline simulators now also include 3D irregular and faulted grids, changes to well controls, rock compressibility and gravity segregation, as well as multi-component, multi-phase flow. However, in terms of gas displacement processes, streamline simulators are not yet fully tested. The principal factors of concern here modelling gas displacement processes will be gas compressibility and then gravity segregation of the different phases.

This part of the dissertation discusses in detail one particular solution strategy concerning how pressure is solved for in streamline simulation, and how the pressure solution is used to minimise the difference between finite differences and streamline simulators. Streamline simulation is based on a sequential approach; the pressure solution is calculated for the end of the time step based on the saturations at the beginning of the time step. The boundary conditions are based upon open wells with given rate targets and limits, aquifer modelling, pressure boundaries and flux boundaries defined by the user. The base case assumption in streamline simulation is that the fluid compressibility is basically zero since water is used as the displacing fluid. However, for this research problem using gas this assumption is not valid. How to solve this issue in order to minimise the differences and improve the match will be done by running a series of sensitivity studies for the streamline case, using tuning factors until a reasonable match is obtained. The saturation solution is tuned with the key word TUNEFSSA. The use and conditions for using TUNEFSSA will be 
described in more detail in the following section. The results are presented in Table 6-3 and Figure 6-8 summaries also the results of the key runs and the impact of different values of the parameter 2 used to control the SL simulation. The sensitivities are based upon the following factors and reasoning:

I. Gravity Segregations: This parameter is used to determine whether or not gravity effects are included in the saturation solver. If it is set to zero, gravity effects are not included. If it is set to a positive integer, then gravity effects are included, and the integer value determines the number of sub-iterations used for it in the saturation solver. Two values have been tested for this, either 1 or 2. The results showed that there was no affect at all between 1 and 2. So, a value 1 was used in all modelling work.

II. Number of streamlines: This parameter key word is called StreamDens. It increases or decreases the number of streamlines used by the saturation solver. The number of streamlines used is the default number calculated by the SL simulator, multiplied by StreamDens. As the speed of the simulator is partly dependent on the number of streamlines, this can be used and scaled via similar tuning options mentioned above. In order to enhance the speed of the simulation, the multiplier in the tuning option TUNEFSSA should be less than 1.0. The use and conditions for using TUNEFSSA will be described in more detail as part of the discussion on sensitivities, but the fact that the number of streamlines needs some independent consideration remains valid. Figure 5-5 summaries the results of the key runs and the impact of different values of the parameter 2 used to control the SL simulation. As can be seen from Figure 6-5, the parameter 2 (StreamDens) number of streamlines used by the saturation solver plays a large role as it has the highest impact of the results based upon the results achieved. The value 0.7 appears to be the most practical one to use.

III. Number of streamlines used in the cell: This parameter key word is called StreammapNS. It activates the streamline mapping of the saturation. The recommended value is zero and the default is zero.

IV. Number of sampling points: This parameter key word is called StreammapNi. It activates the number of streamlines and number of sampling points in one single cell. The recommended value is zero and the default is zero.

V. Add Streamlines: This parameter key word is called Addlines. After streamlines through the generated start points have been traced, the streamline simulator will look for the cells that have not yet been visited by streamlines, in order to add other streamlines when necessary. This 
additional process can be switched off by setting TUNEFSSA parameter five to NO. In this work, YES was used as an option of adding more line whenever was needed in order to fulfil the aim of this work to achieve maximum accuracy using SL. Therefore, the option was chosen to ensure that all cells have been visited by streamlines.

VI. Reserved for Future use: This key word been left as it is by the developer and the default is No.

VII. FluxMult: The streamline algorithm is an interactive method where an entry point can be traced to an exit point for every grid cell. The time of flight variable and the flow rate, in the case of compressible fluid, are updated for the exit point given the values at the entry point, and the process will stop whenever a source/sink is reached or the flow rate is less than a minimum. This minimum flow rate can be controlled and scaled using TUNEFSSA parameter seven. In the case of a compressible fluid such as gas, the source or sink condition will be definitively different than that for an incompressible fluid. The default value was used on this work in order to ensure that more streamlines are traced for maximum accuracy, and the default value is 1 .

VIII. StarType: This parameter is used to determine where to start tracking of streamlines, and there are three possible values that can be used. These values are: Both, INJ and PROD. Default is Both, and the default was used in this work.

A series of sensitivities were performed using the tuning options described in the previous section. Table 6-3 and Figure 6-5 show the sensitivity results on parameter 2, the impact on oil production rate, how the rate improved and the difference. The sensitivities mainly were done using the options TUNESFSSA. 
Table 6-2: Streamline simulation control output tuning parameters:

\begin{tabular}{|c|l|c|}
\hline TUNESSFA & \multicolumn{1}{|c|}{ Parameter } & $\begin{array}{c}\text { Value } \\
\text { used }\end{array}$ \\
\hline 1 & Gravity Segregation, Default is 1 & 1 \\
\hline 2 & $\begin{array}{l}\text { StreamDens, number of streamline used by } \\
\text { saturation solver }\end{array}$ & 0.7 \\
\hline 3 & $\begin{array}{l}\text { StreammapNs, number of streamlines used in a cell } \\
\text { Default is 0, recommended 0 }\end{array}$ & Default \\
\hline 4 & $\begin{array}{l}\text { StreammapNi, Number of sampling points Default } \\
\text { is 0, recommended 0 }\end{array}$ & Default \\
\hline 5 & $\begin{array}{l}\text { Addlines, When set, FrontSim checks whether each } \\
\text { cell has been visited by at least one streamline }\end{array}$ & Yes \\
\hline 6 & $\begin{array}{l}\text { Reserved for future use. } \\
\text { No } \\
\text { flux, Default is 1 multiplier adjusts the threshold }\end{array}$ & $\begin{array}{c}\text { Default } \\
\text { (1) }\end{array}$ \\
\hline 8 & $\begin{array}{l}\text { StartType:where to start tracking streamline, either } \\
\text { INJ, PROD, or Both }\end{array}$ & $\begin{array}{c}\text { Default } \\
\text { (Both) }\end{array}$ \\
\hline
\end{tabular}

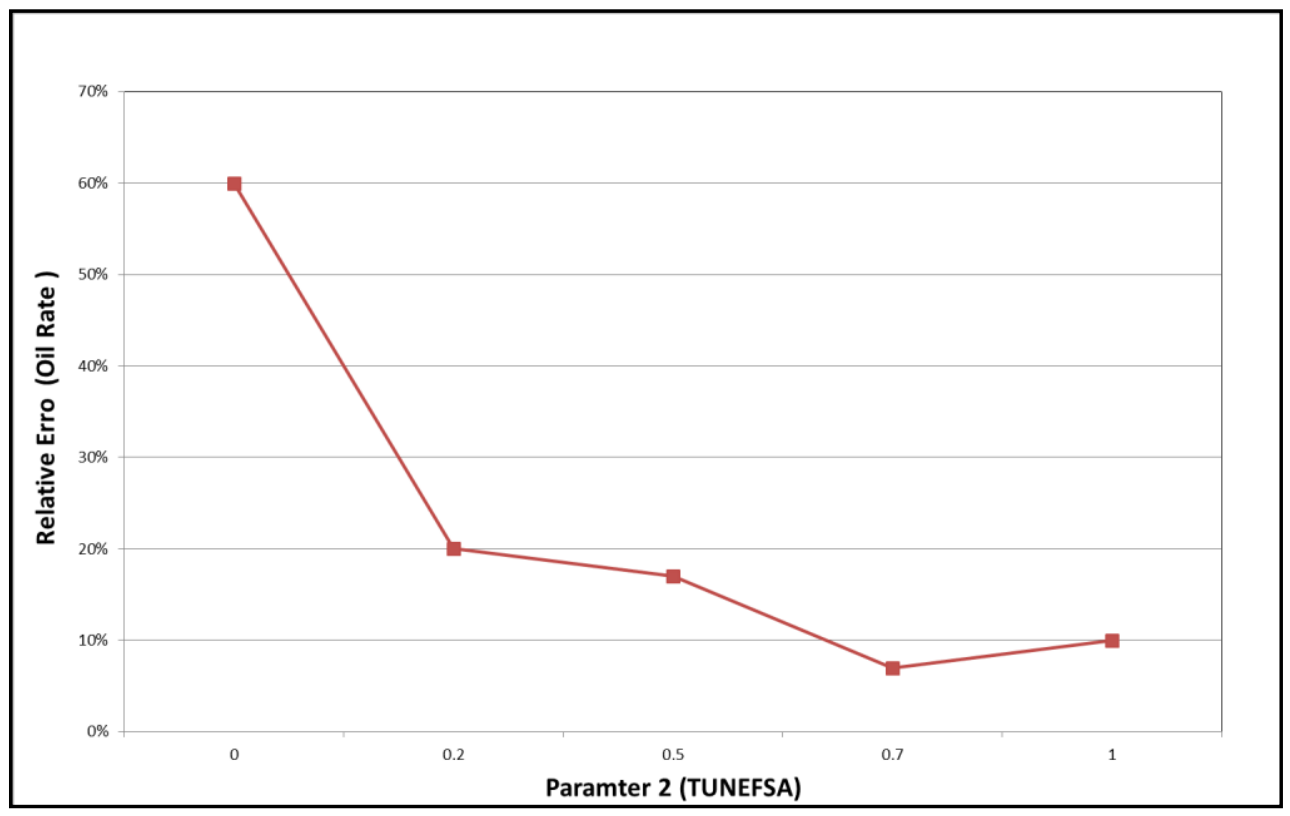

Figure 6-5: Sensitivity Results of the SL Tuning Parameters with the Relative Error (Oil Rate).

Figure 6-6 shows the sensitivity results on the oil production using different tuning parameter values, and the comparisons are always made against the FD simulation results. 


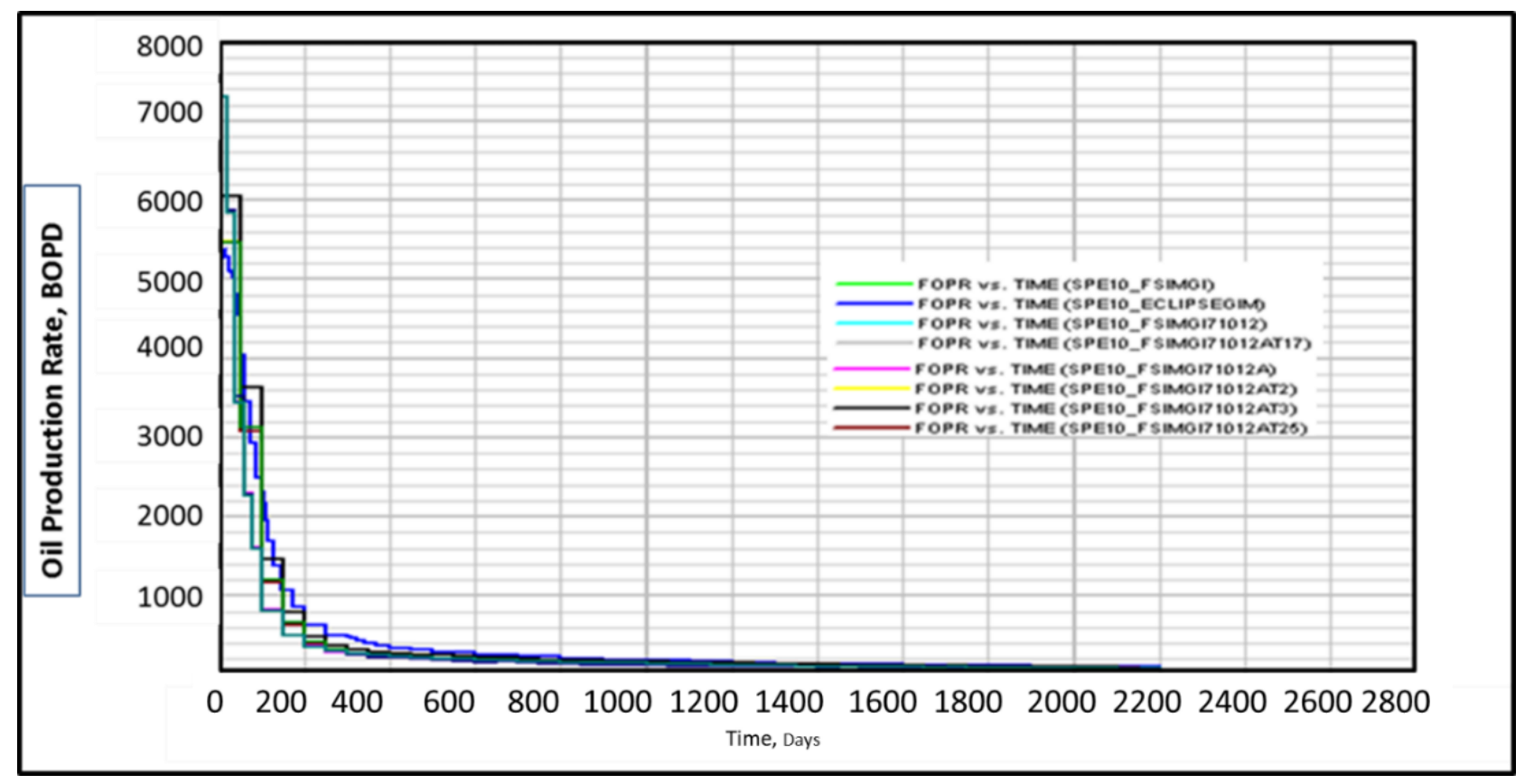

Figure 6-6: Sensitivity results (Oil production profile) for FD and SL simulation.

Figure 6-7 shows the same results in a smaller time scale, for the first 200 days, at the top (A), and or the first 500 days, at the bottom (B). 

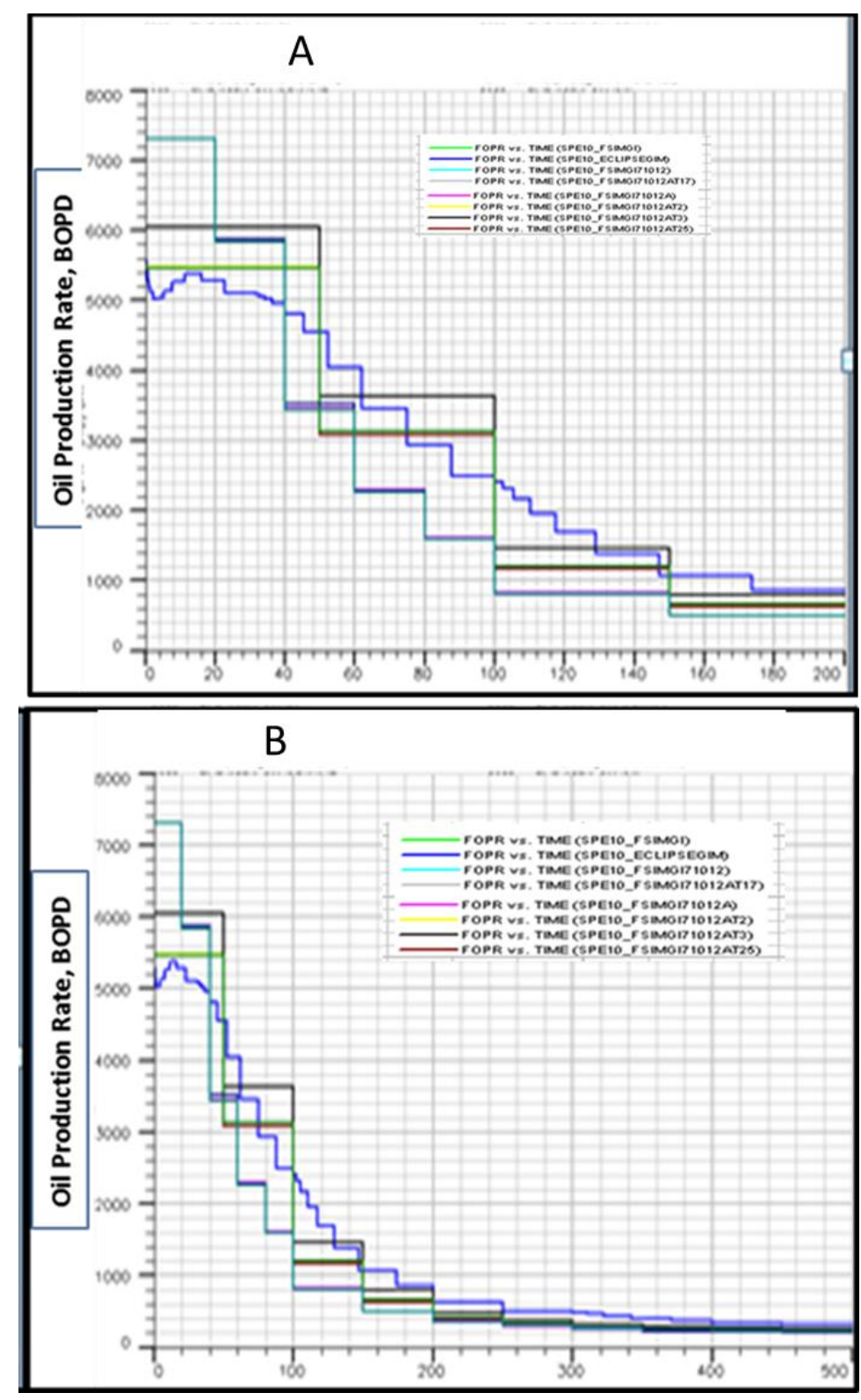

Figure 6-7: Sensitivity results (Oil production profile) for FD and SL simulation.

As can be seen from the above plots, a very reasonable match was achieved, with minor differences between both streamline and finite difference simulators.

\subsection{Final Tuning on Time step control}

As per the results presented above, the final acceptable degree of match has not yet been achieved. It was concluded that SL time steps calculations and reporting are still not capable to match the finite 
difference results, as the finite difference method is able to report and handle any required time steps. To control this and improve the streamline calculation, an additional tuning, improvements and fine controlling on time steps is still required to reach an acceptable level of match. Therefore, in order to enhance the match, additional control parameters were introduced from FD to SL and sensitivities were performed. These parameters were mainly to add more control in time steps and initial rates in order to make sure that both FD and SL have similar controls. In this part, all the constraints such as initial oil rate per well, maximum liquid rates and water cut used in the FD simulation are similar to the ones used in the SL simulation. An improvement on oil rate and total cumulative oil produced was noticed and all other parameters as shown in the following graphs. Figure 6-8 shows the latest results of the streamline simulation compared with finite difference simulation results.

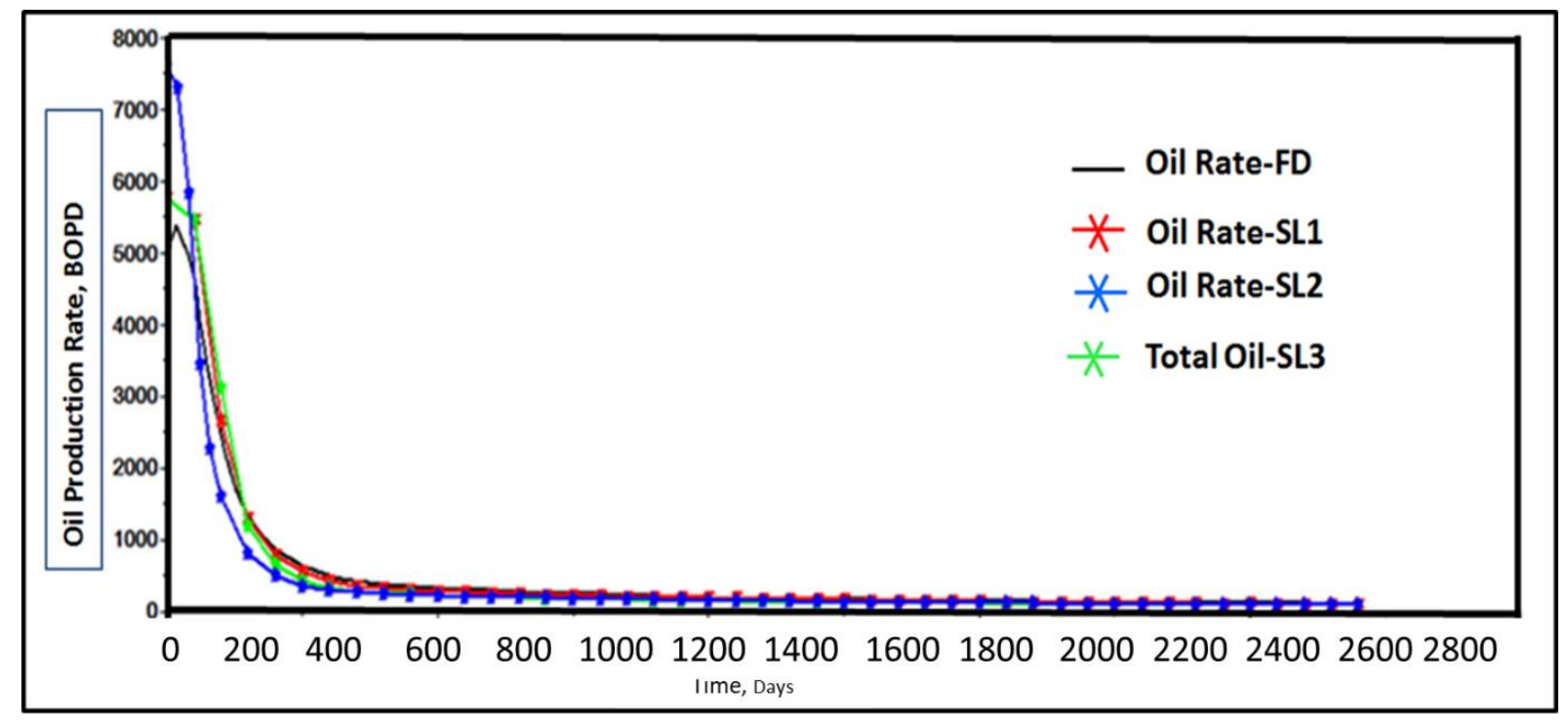

Figure 6-8: Sensitivity results (Oil production profile) for both FD and SL simulation

The above figure represents a comparison between FD and SL simulation. The left-hand side access represents oil rates for three cases: case 1, FD simulation (light blue colour), case2, Streamline old model (blue colour) and case 3, latest Streamline simulation model (green colour). The right-hand side curves represent the total oil produced for the three cases; case 1, FD simulation (Pink), case 2, old Streamline model (Yellow) and case 3, Latest streamline simulation model (black). As noticed in the above graph, a high degree of match was obtained from the streamline simulation model and the performance now is 
reasonably close to the one predicted from FD simulation. The same graphs for shorter periods of time are presented next.

Figure 6-9 show the sensitivity results and comparisons of the oil production rates on the left axes, total oil produced on the right axes versus time using streamline simulation with final tuning and controlling parameters, and compares them with Eclipse Finite Difference on smaller time scales (200 days).

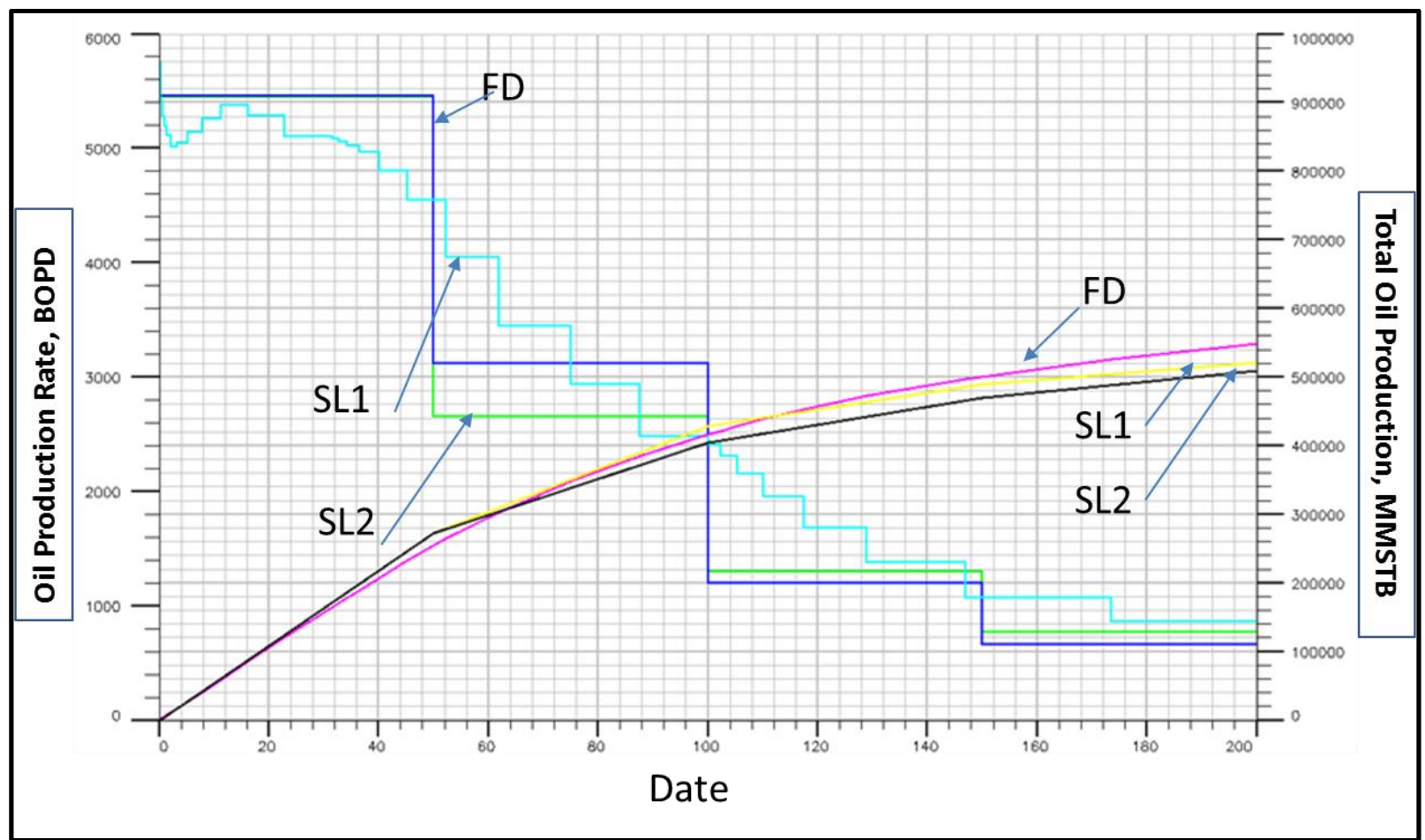

Figure 6-9: Sensitivity results (Oil production profile) for both Finite Difference and Streamline simulation for the first 200 days.

Figure 6-10 show the same sensitivity results and comparisons as presented in figure 6-9 for the first 500 days period. 


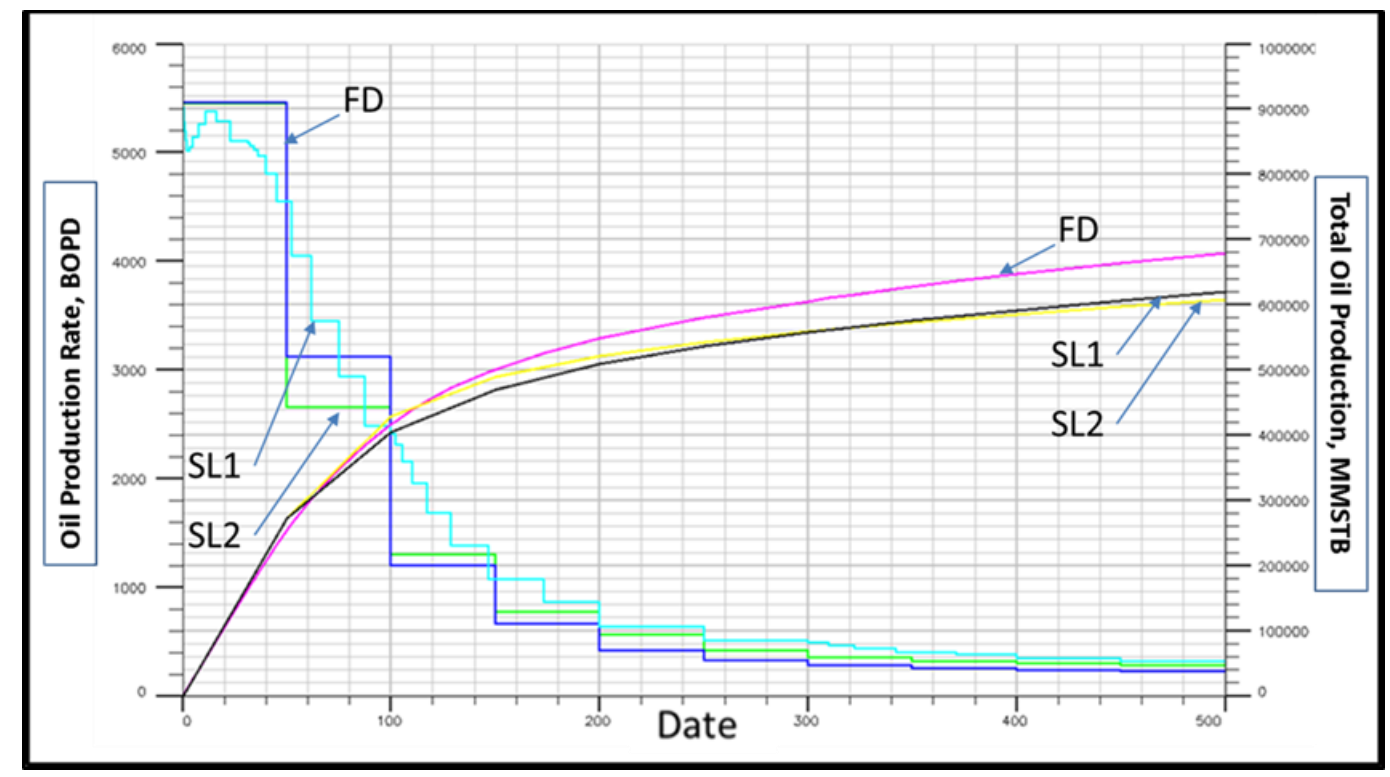

Figure 6-10: Sensitivity Results (Oil production profile) for both Finite Difference and Streamline simulation for the first 500 days.

Figure 6-11 shows the oil saturation grids at zero time step and, as can be seen from the graph, there are no changes in oil saturation as there is still no production. 


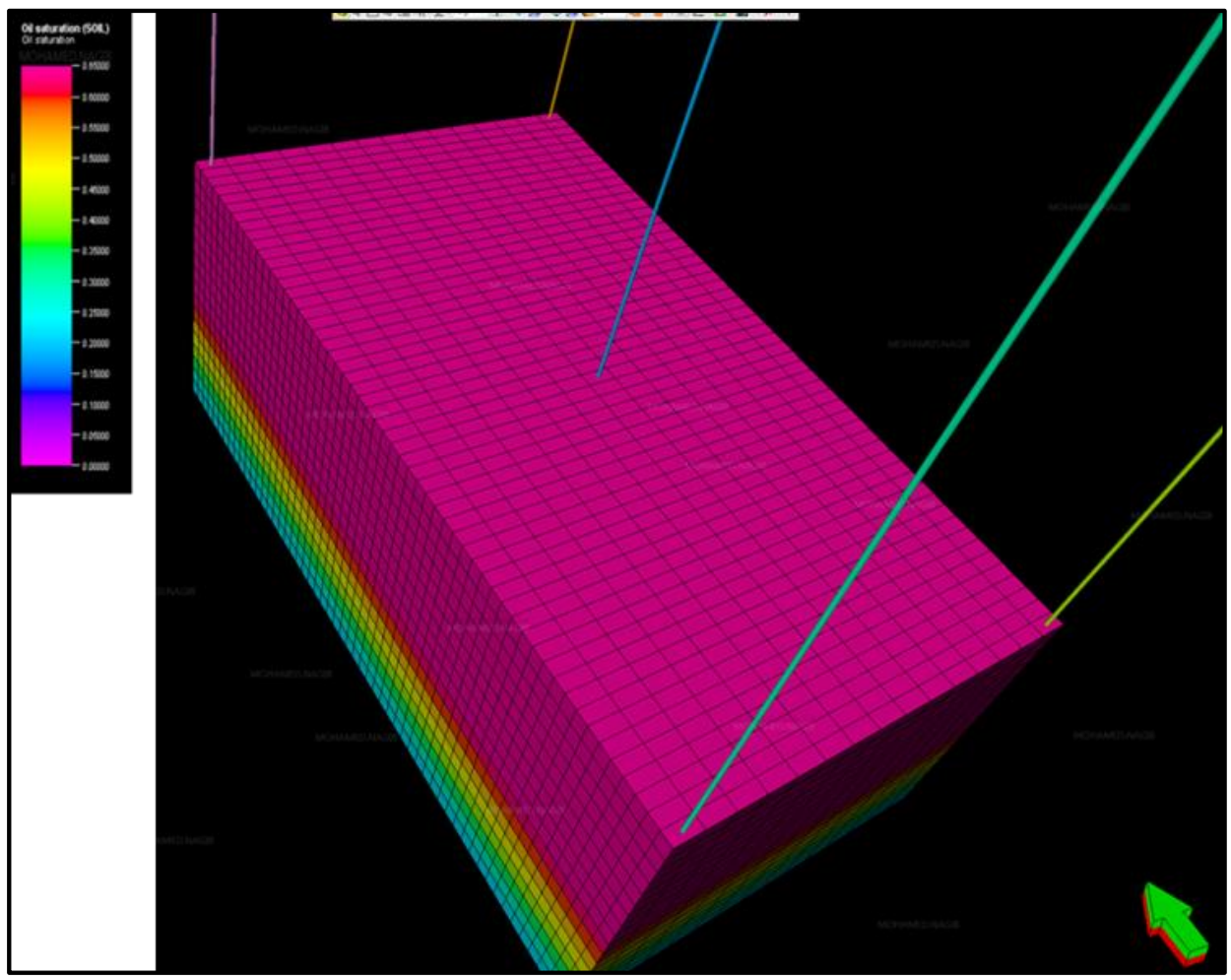

Figure 6-11: Streamline Simulation Oil saturation Grid at Initial time step before production.

The oil saturation grid at the second time step is presented in Figure 6-12. In the graph and on this time step, gas injection has just started as shown in the middle of the grid and, in the meantime, oil is starting to be produced as well. A change in the oil saturation starts to appear.

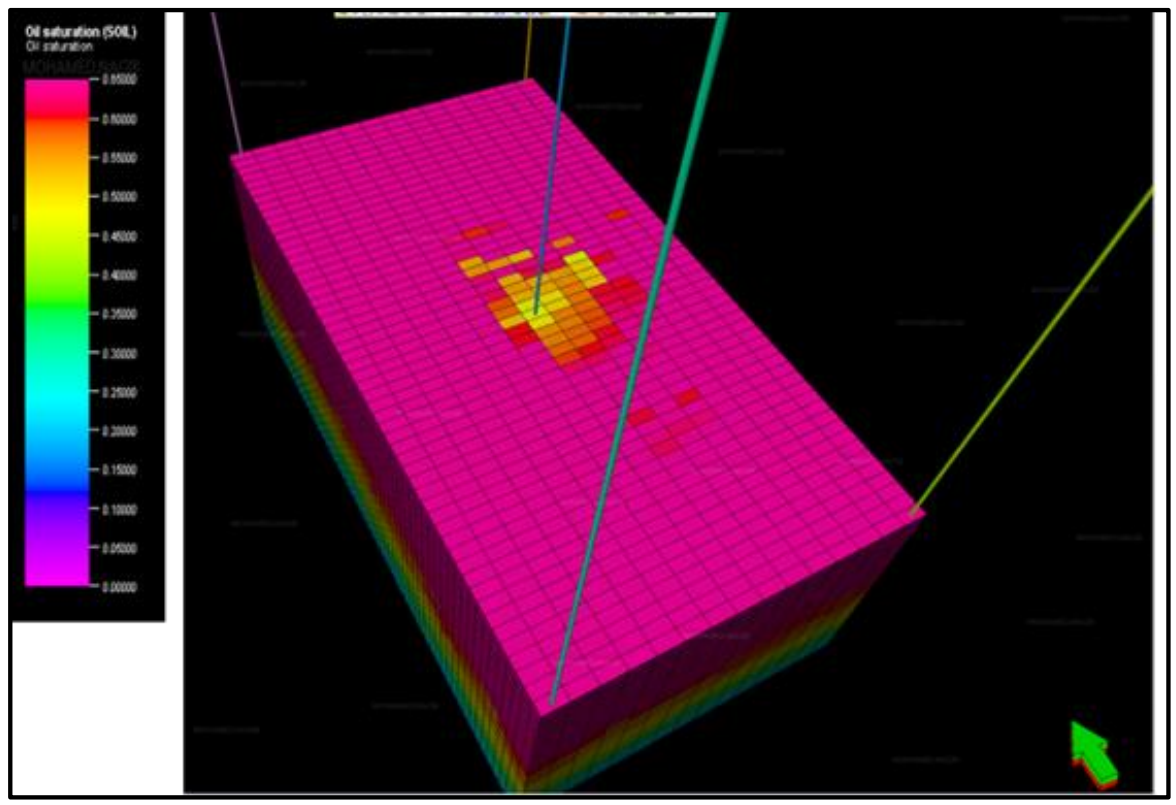


Figure 6-12: Streamline Simulation Oil saturation Grid at the first time step just after start of production.

The oil saturation grid at the end of the simulation time is presented also in figure 6-13; on this time step we can notice that the change in the saturation as a result of displacing the oil by gas injection throughout the injector is in the middle of the grid.

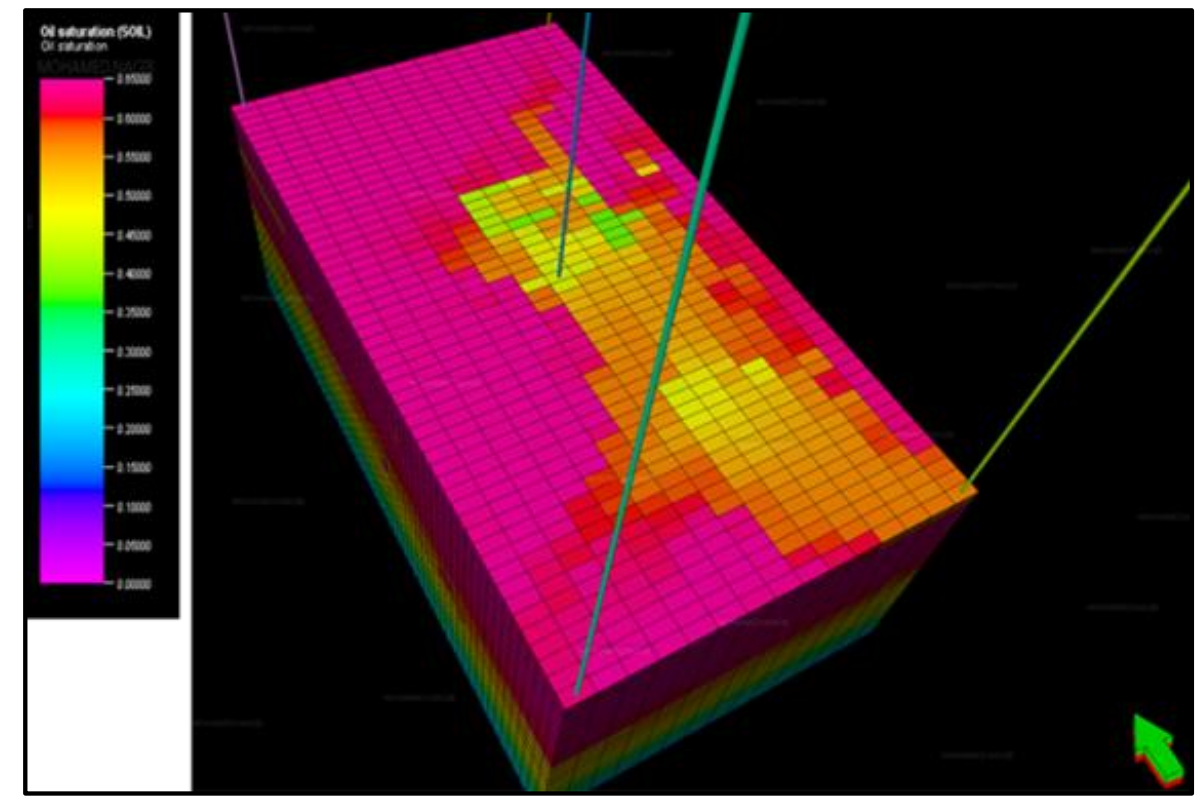

Figure 6-13: Streamline simulation Oil saturation Grid at the end of Simulation.

\subsection{Critical Analysis of the Results}

A special program was developed in order to properly define and quantify the difference between finite difference and Streamline simulations, and it was mainly used as a post processor for the simulation results and mainly to quantify and plot the error difference between the two separate runs. Another development was further added on how old and new sets of runs can be evaluated simultaneously in order to show the comparison before and after these sensitivities. The following series of graphs present a full evaluation and comparisons between FD simulation and Streamline simulation for gas displacement processes. This evaluation tool was developed based upon the following assumptions; given a FD 
simulation parameter $\mathrm{A}$ and a corresponding streamline simulation parameter $\mathrm{B}$, the relative error difference will be based on the following equation;

$$
\mathrm{Y}=(\mathrm{A}-\mathrm{B}) / \mathrm{A}
$$

The Program creates a new vector for each parameter calculated on the dynamic simulation such as oil production rates, oil production total, gas production rates, gas production totals, gas injection rates, gas injection totals and field pressures. We are then able to evaluate, assess and quantify the error difference between FD and Streamline simulation.

\subsection{Initial Runs vs. Final Tuned Model}

Figure 6-14 below presents the percentage differences between finite differences and streamline simulation for the oil production rate versus time for the two sets of data. As can be seen from the plot, for the initial set model run, both (FD \& SL), the relative error has an average value of $+/-50 \%$ in oil rate. However, the final matched and tuned runs represent a $+/-5 \%$ error difference. The same observation can be noticed in Figure 6-15 for cumulative oil production.

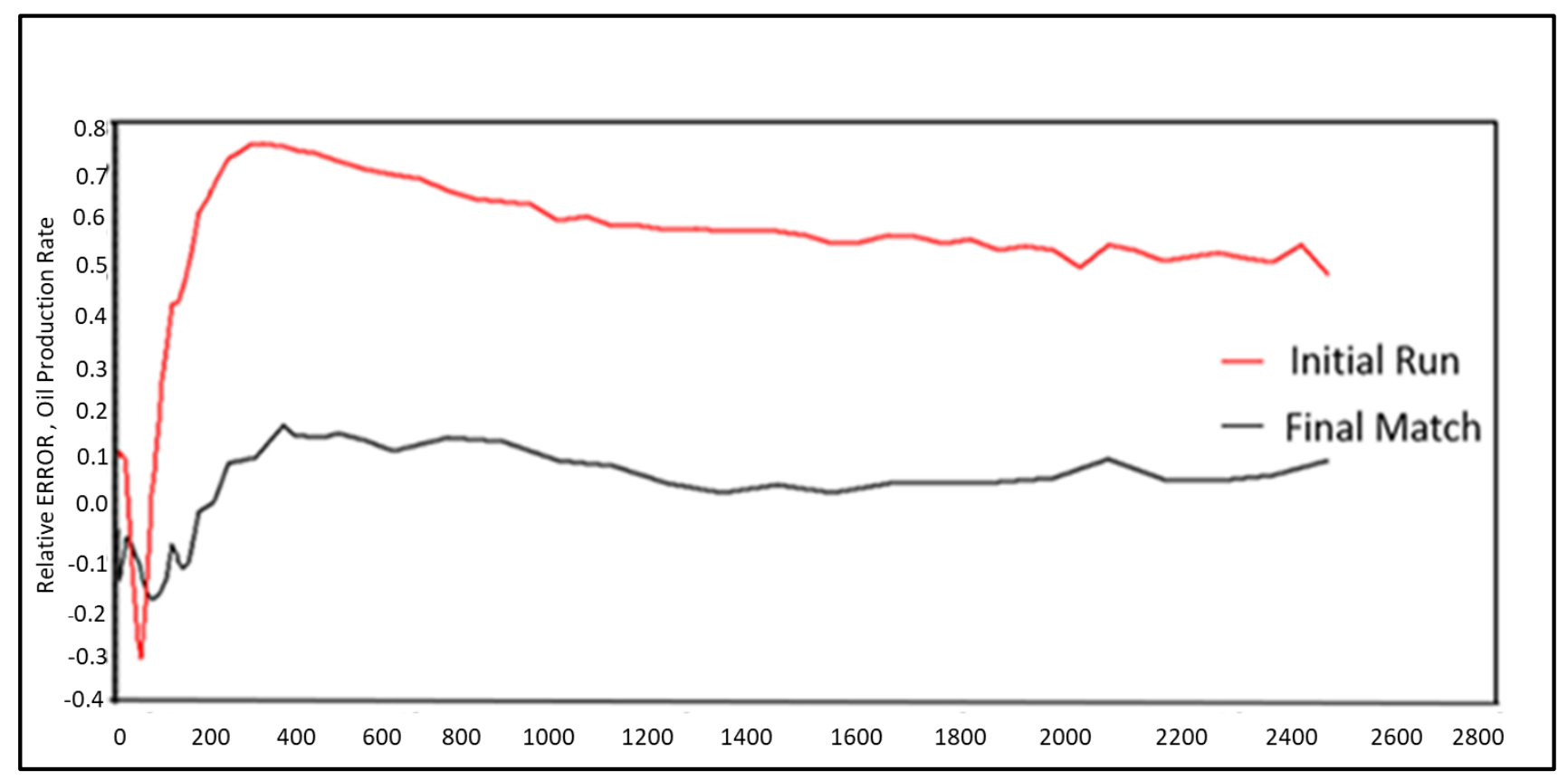

Figure 6-14: Field Oil Production Rate Differences (old and final) Simulation cases. 


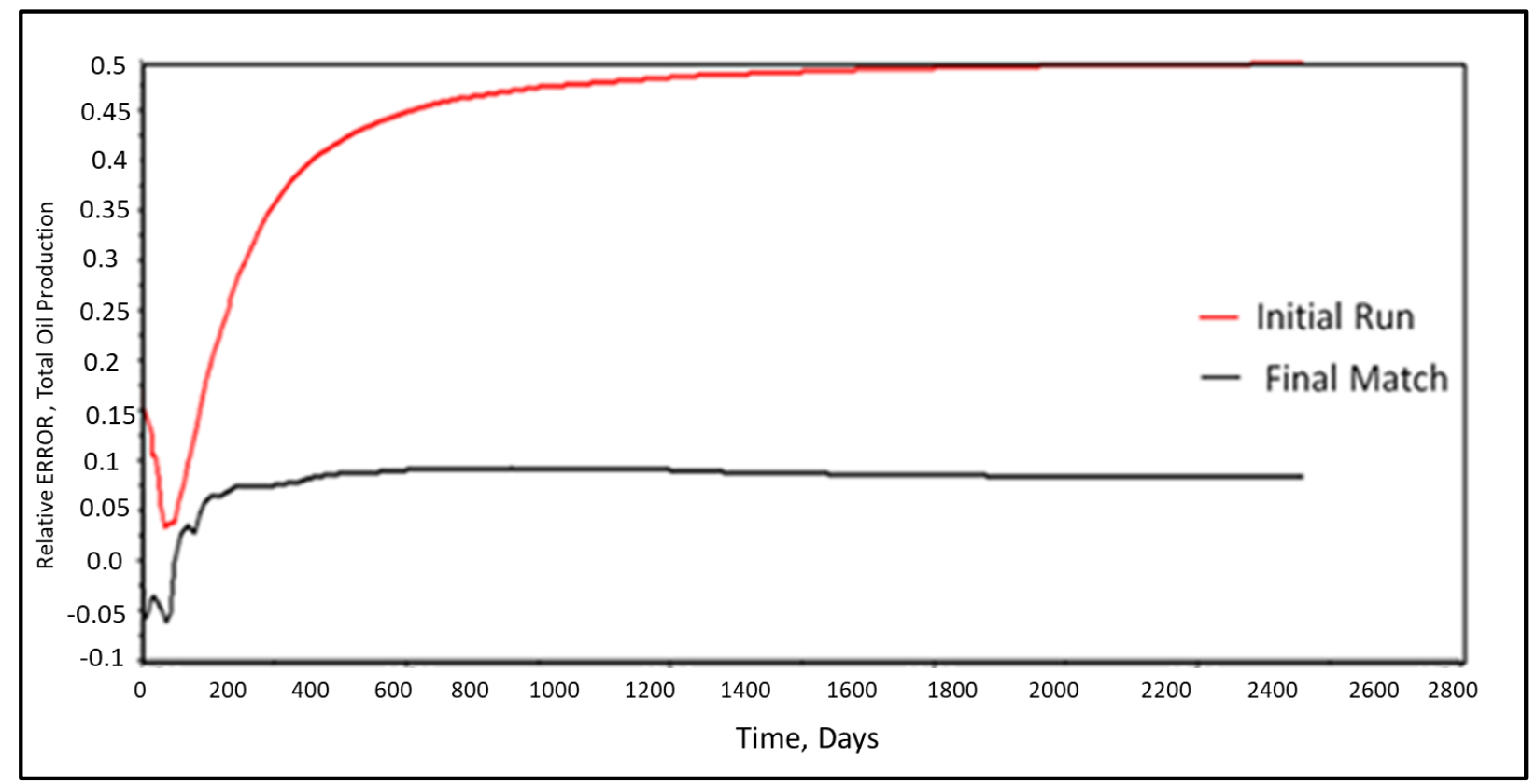

Figure 6-15: Field Oil Production Total Relative Error (Initial and final) Simulation cases.

Figure 6-16 presents gas production rates in both models at initial models set up vs. the final tuned model. A general observation can be noticed that in the initial run the relative error is approximately +/$4 \%$. However, the final tuned model represents negligible differences.

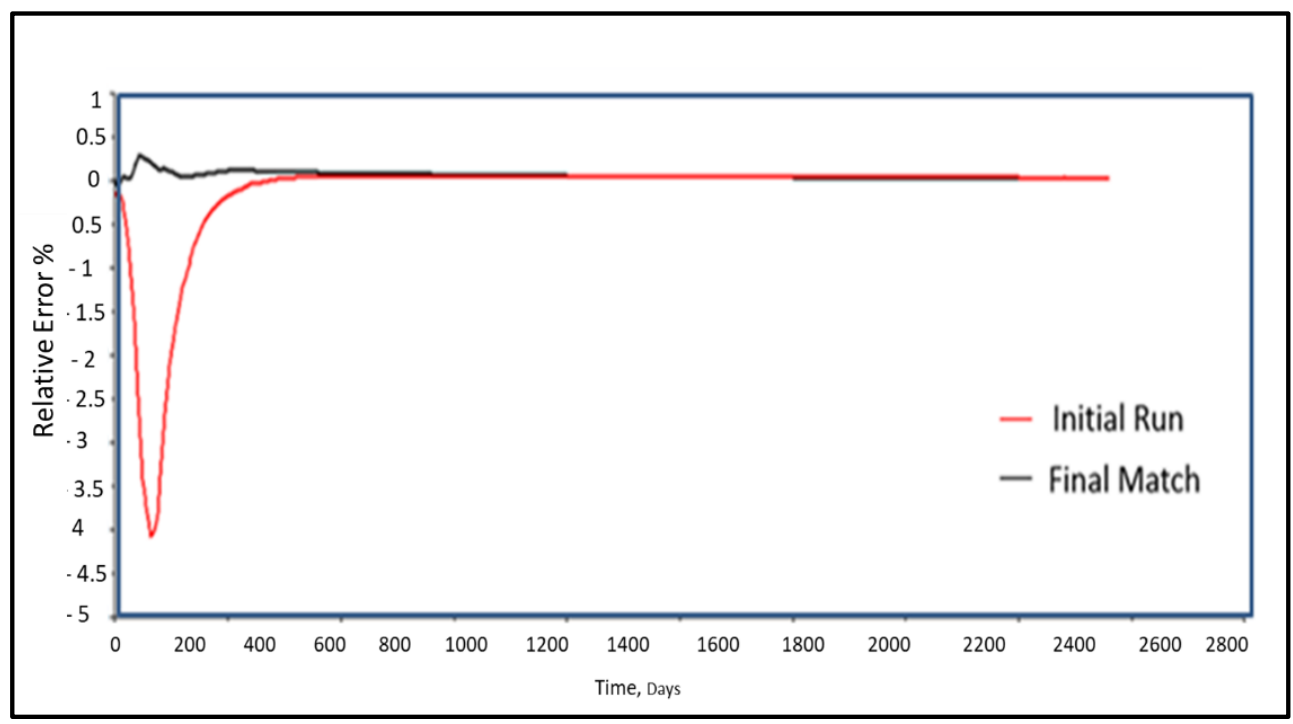

Figure 6-16: Field Gas Production Rate Relative Error (Initial and final) Simulation cases. 
Figure 6-17 presents the relative error for gas injection rate parameter. As can be seen from the graph, the initial run initial relative error was $+/-25 \%$. However, the final match relative error is almost zero.

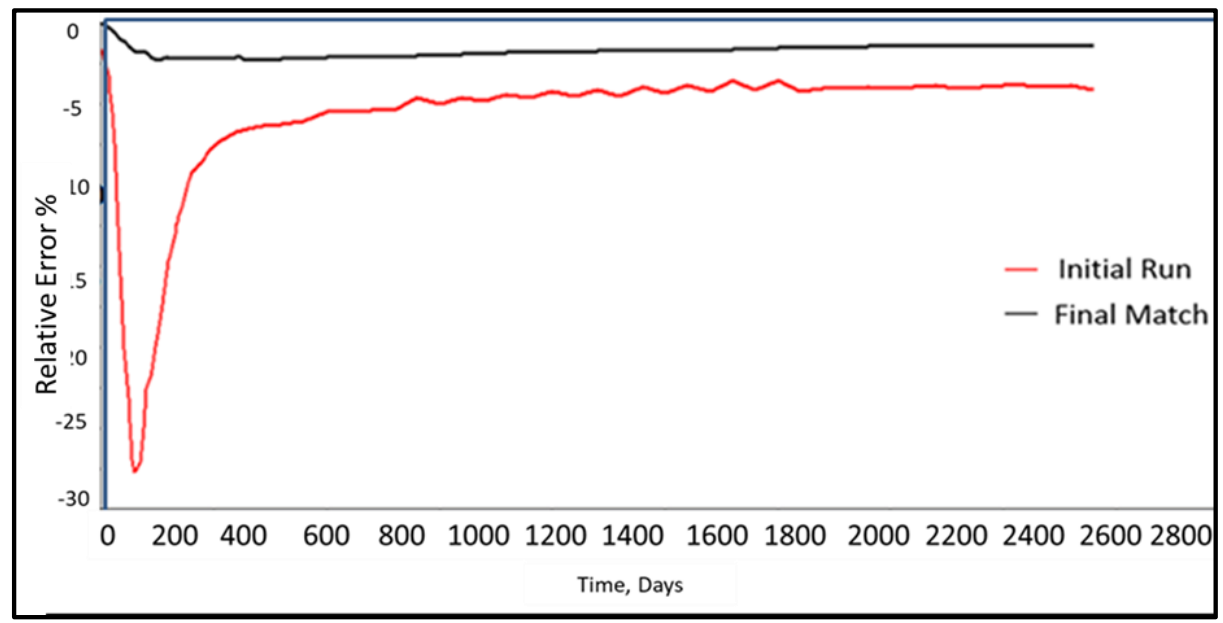

Figure 6-17: Field Gas Injection Rates Relative Error (Initial and final) Simulation cases.

Figure 6-18 presents the relative error for the water production parameter. As can be seen from the graph, the initial run initial relative error was $+/-1.5 \%$. However, the final match relative error is almost zero.

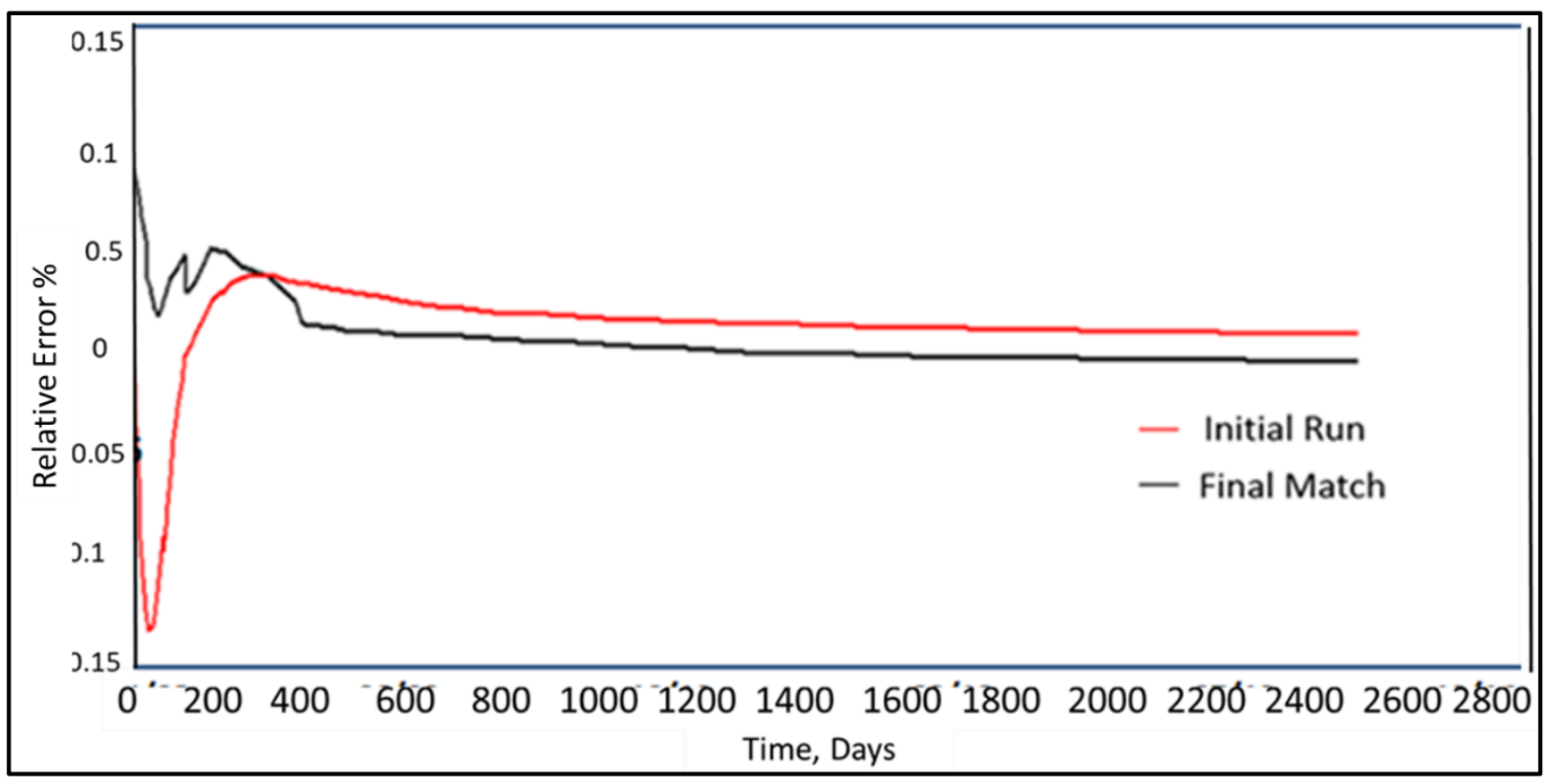

Figure 6-18: Field water production rates Relative Error (Initial and final) Simulation cases. 
Figure 6-19 presents the relative error for the water cut parameter. As can be seen from the graph, the initial run initial relative error was $+/-15 \%$. However, the final match relative error is almost zero.

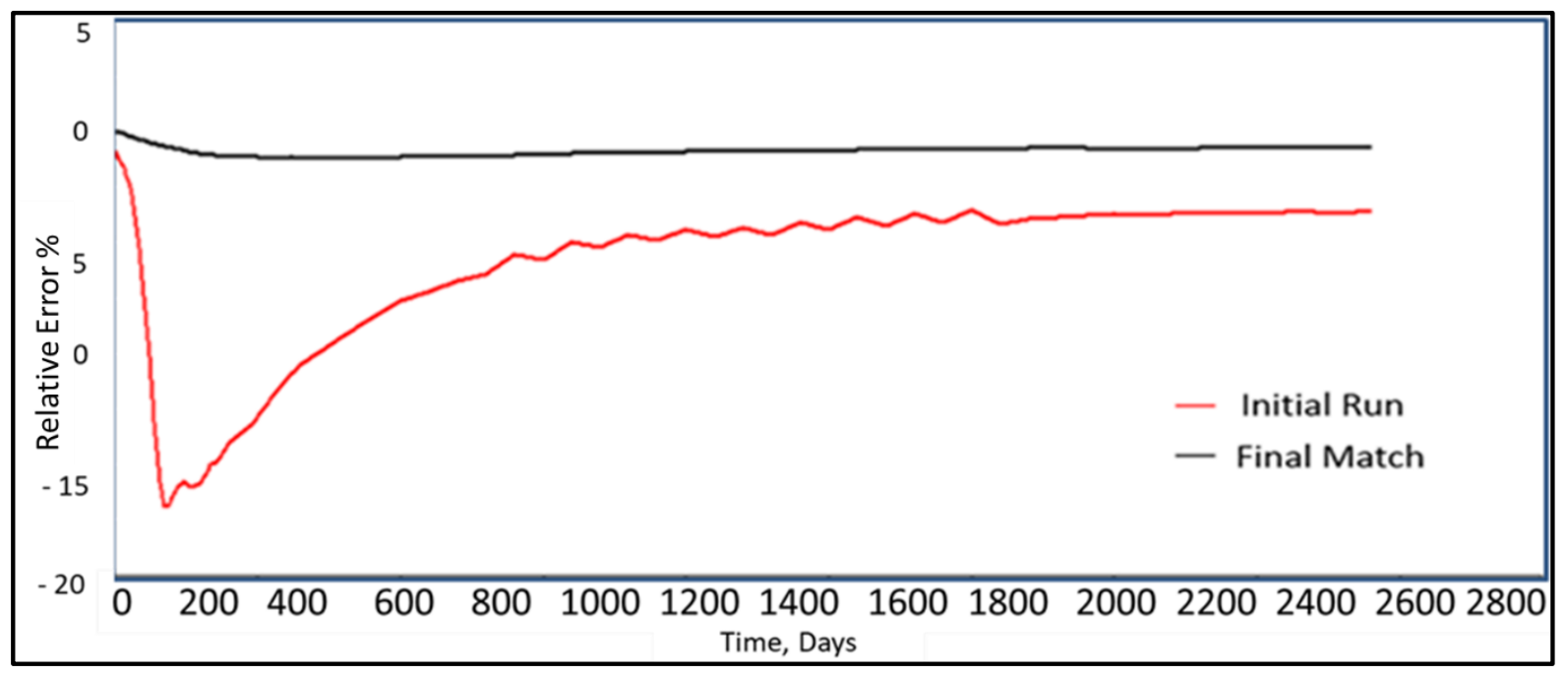

Figure 6-19: Field water cut Relative Error (Initial and final) Simulation cases.

Figure 6-20 presents the relative error for the reservoir pressure parameter. As can be seen from the graph, the initial run initial relative error was $+/-10 \%$; this high relative error was only observed on the first few time steps, then this high relative error is diminished and becomes minimum. However, the final match relative error is almost zero. 


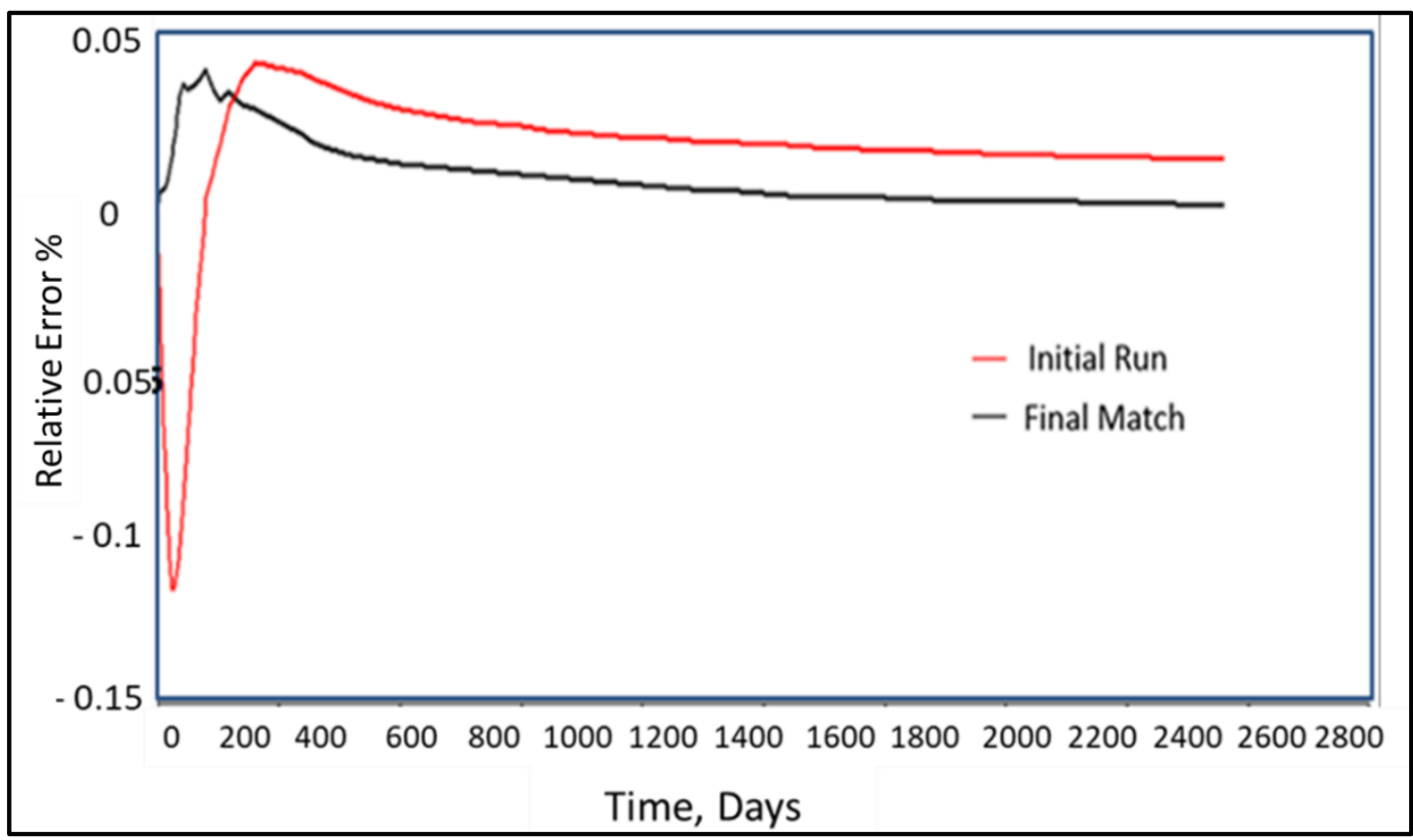

Figure 6-20: Field Pressures Relative Error (Initial and final) Simulation cases.

\subsection{Detailed 3D Analysis (Base Case Gas Injection)}

The following section will present detailed 3D analysis, the analysis carried out in order to see if there are differences in 3D domain in terms of oil saturation at both first- and last-time steps.

With regards to the base case gas injection model (Black oil FD Vs SL Simulation), figure 6-21 below shows the 3D oil saturation grid at the first-time step on the left and on the right shows at the end of simulation for FD black oil case.

When comparing both models in Figure 6-21, slight differences can be noticed in terms of oil saturation changes. 


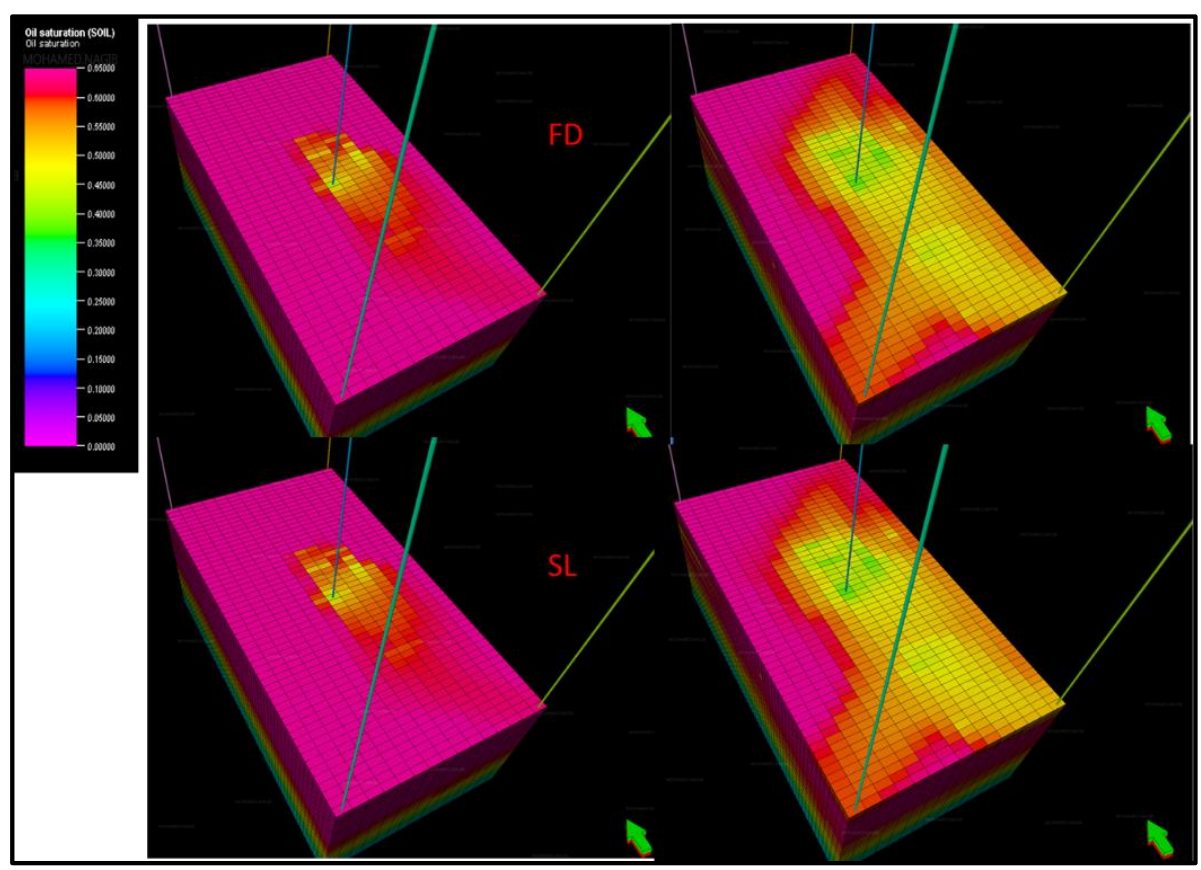

Figure 6-21: First- and Last-time steps 3D Oil saturation Grid (Base Case Model)

Sliced 3D oil saturation grids are presented in figures 6-22 for both FD and SL base case gas injection model. Similar conclusion obtained as well from this comparison as concluded earlier in 3D grids above.

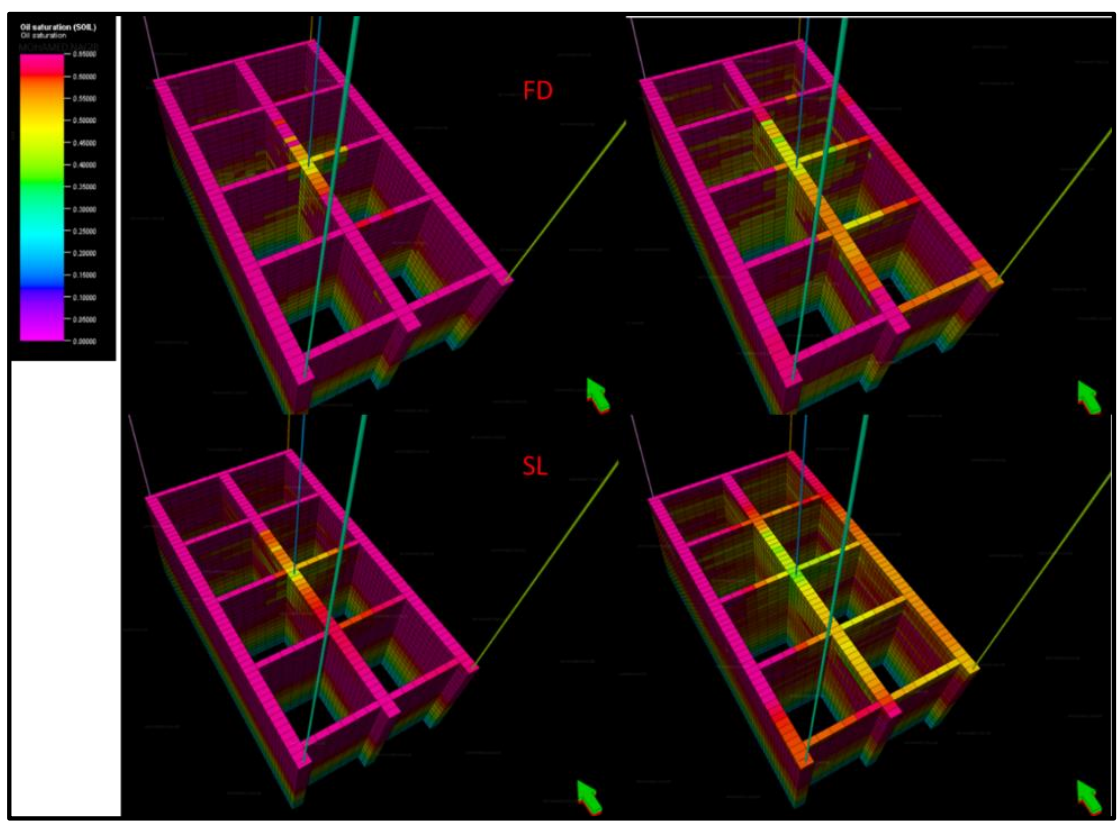

Figure 6-22: First- and Last-time steps Sliced 3D Oil saturation Grid (Base Case Model) 
Cross section 3D oil saturation grids are presented in figures 6-23for both FD and SL base case gas injection model. Similar conclusion obtained as well from this comparison as concluded earlier in 3D grids above.

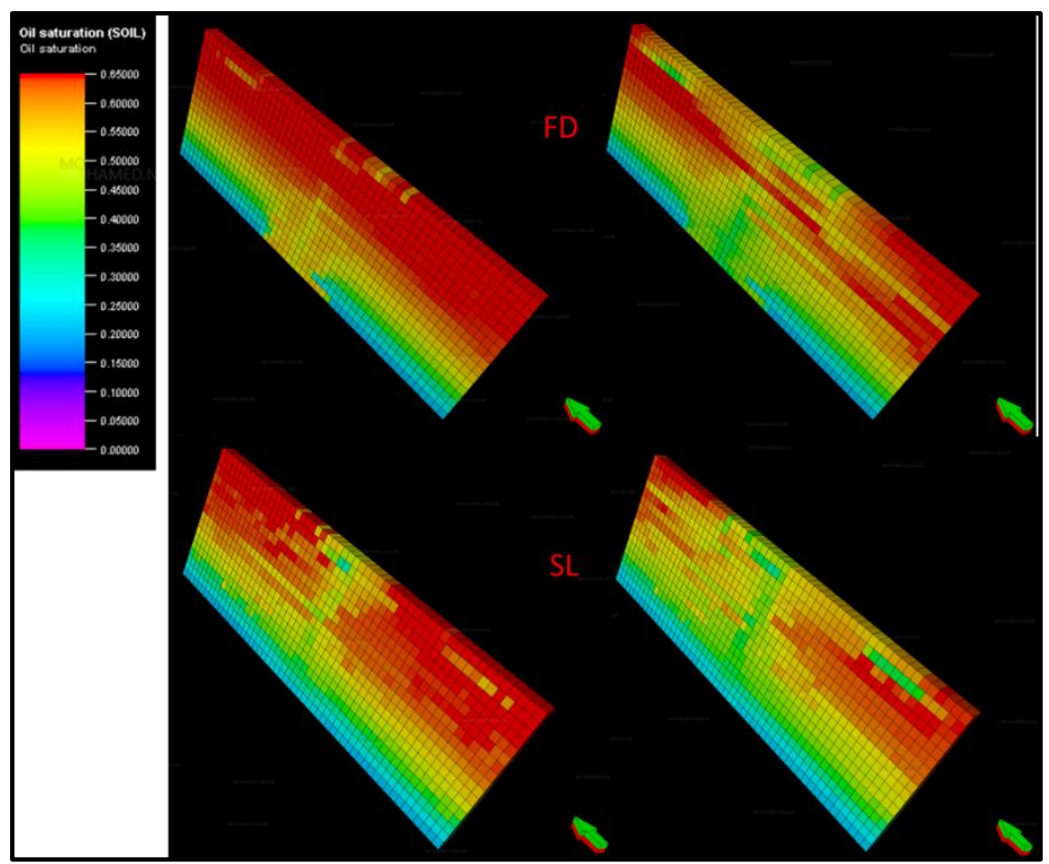

Figure 6-23: First- and Last-time steps of Cross Section 3D Oil saturation Grid (Base Case Model)

A similar analysis is done as well for gas saturation. Base case 3D gas Saturations at first- and lasttime steps is presented in figure 6-24.

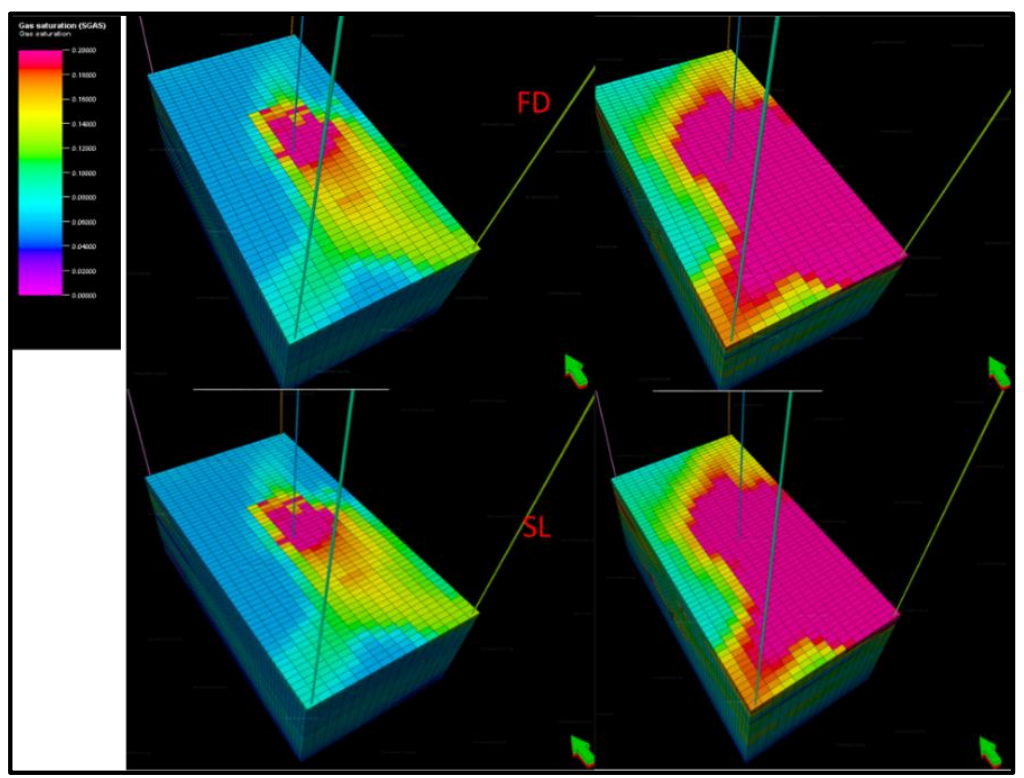

Figure 6-24: First- and Last-time steps of sliced 3D Gas saturation Grid (Base Case Model) 
Sliced 3D gas saturation grids are presented in figures 6-25 for both FD and SL bas case gas injection model. Similar conclusion obtained as well from this comparison as concluded earlier in 3D grids above.

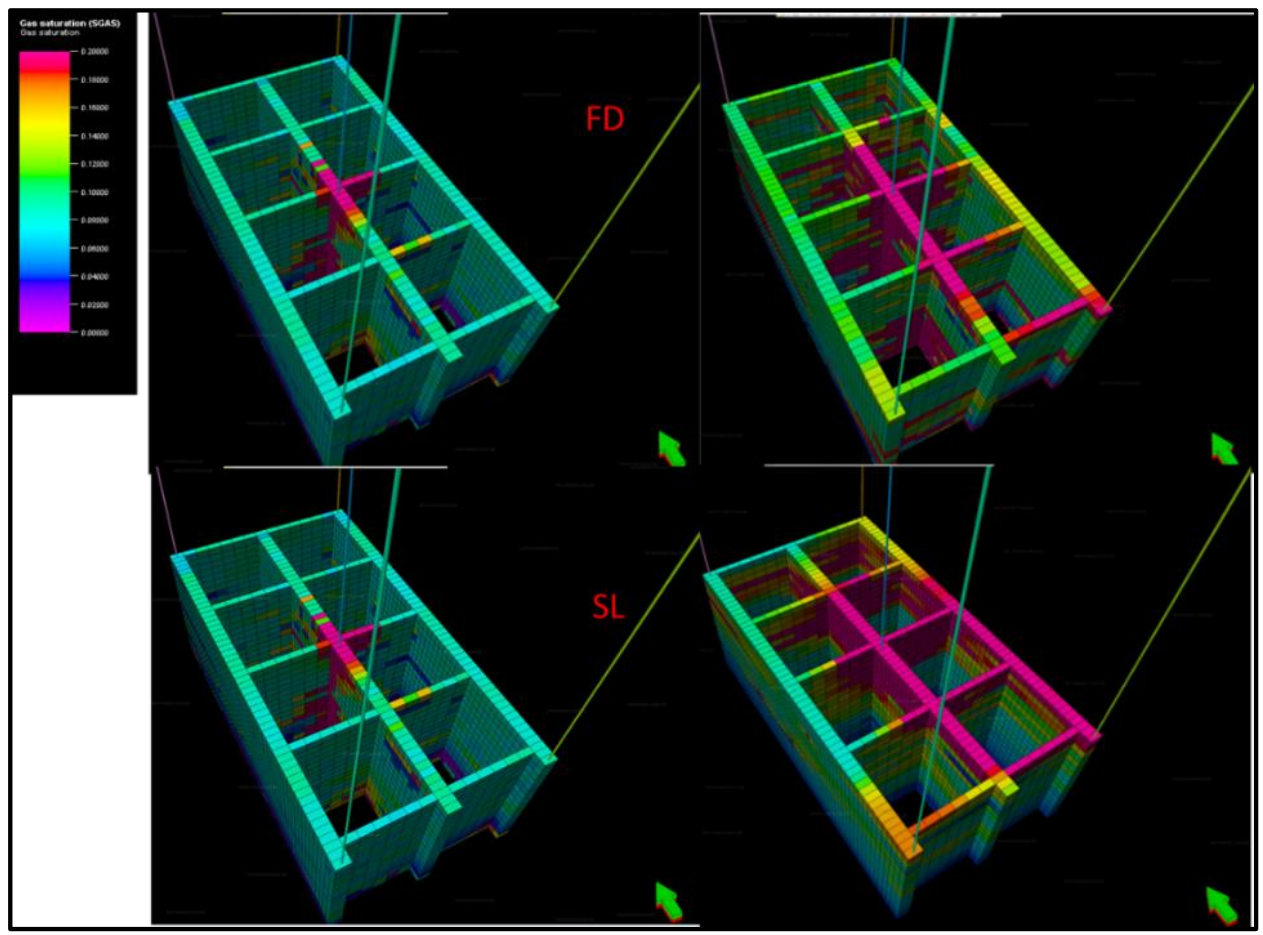

Figure 6-25: First- and Last-time steps of a sliced 3D Gas saturation Grid (Base Case Model)

From the sliced graphs above, similar trends can be noticed inside the 3D Grid as well. Cross 3D gas saturation grids are presented in figures 6-26 for both FD and SL base case gas injection model.

Similar conclusion obtained as well from this comparison as concluded earlier in 3D grids above. 


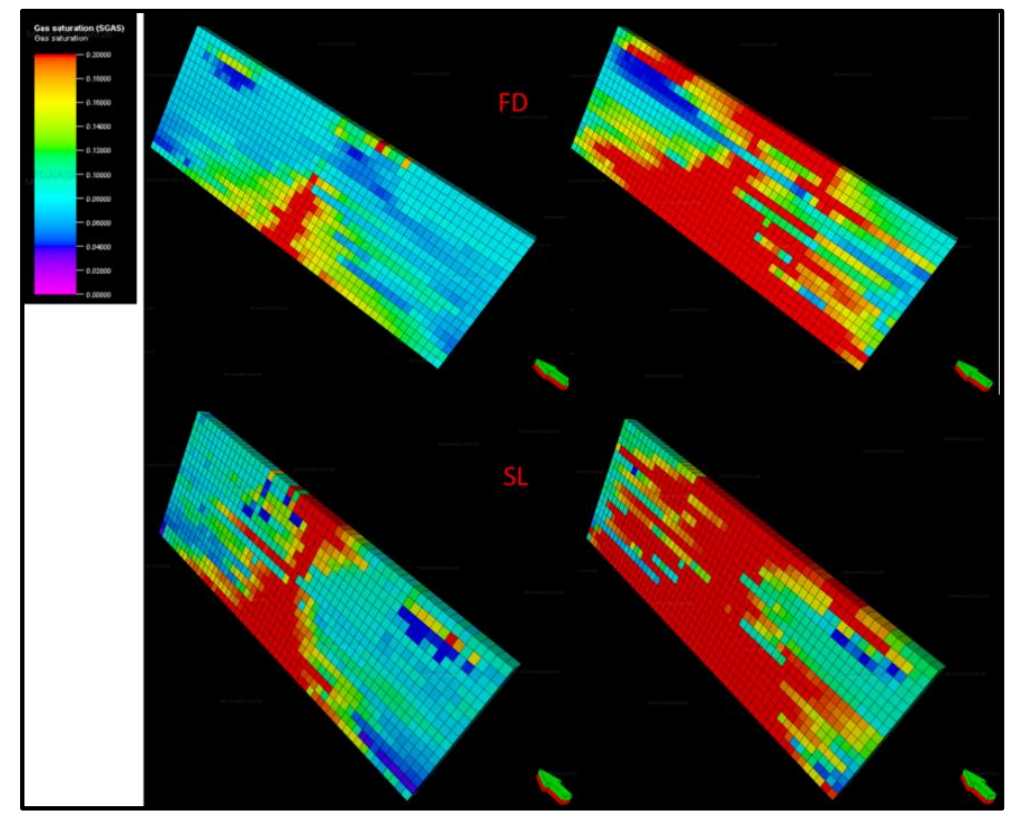

Figure 6-26: First- and Last-time steps of a Cross Section 3D Gas saturation Grid (Base Case Model)

In order to evaluate the sweep as a result of gas injection, a new grid property was generated to Oil Thickness Column (OTC) at the end of the field life and this property calculated as per the following equation:

OTC $=$ Cell Thickness $*$ Oil Saturation

- Oil saturation is the saturation calculated at the end of field life.

The new property calculated for both FD and SL grids at the end of the field life

This OTC calculated as a results of oil saturation change due to gas injection and production starting from initialisation to the end of simulation time. Figure 6-27 shows OTC at the end of field life of both FD and SL models. 


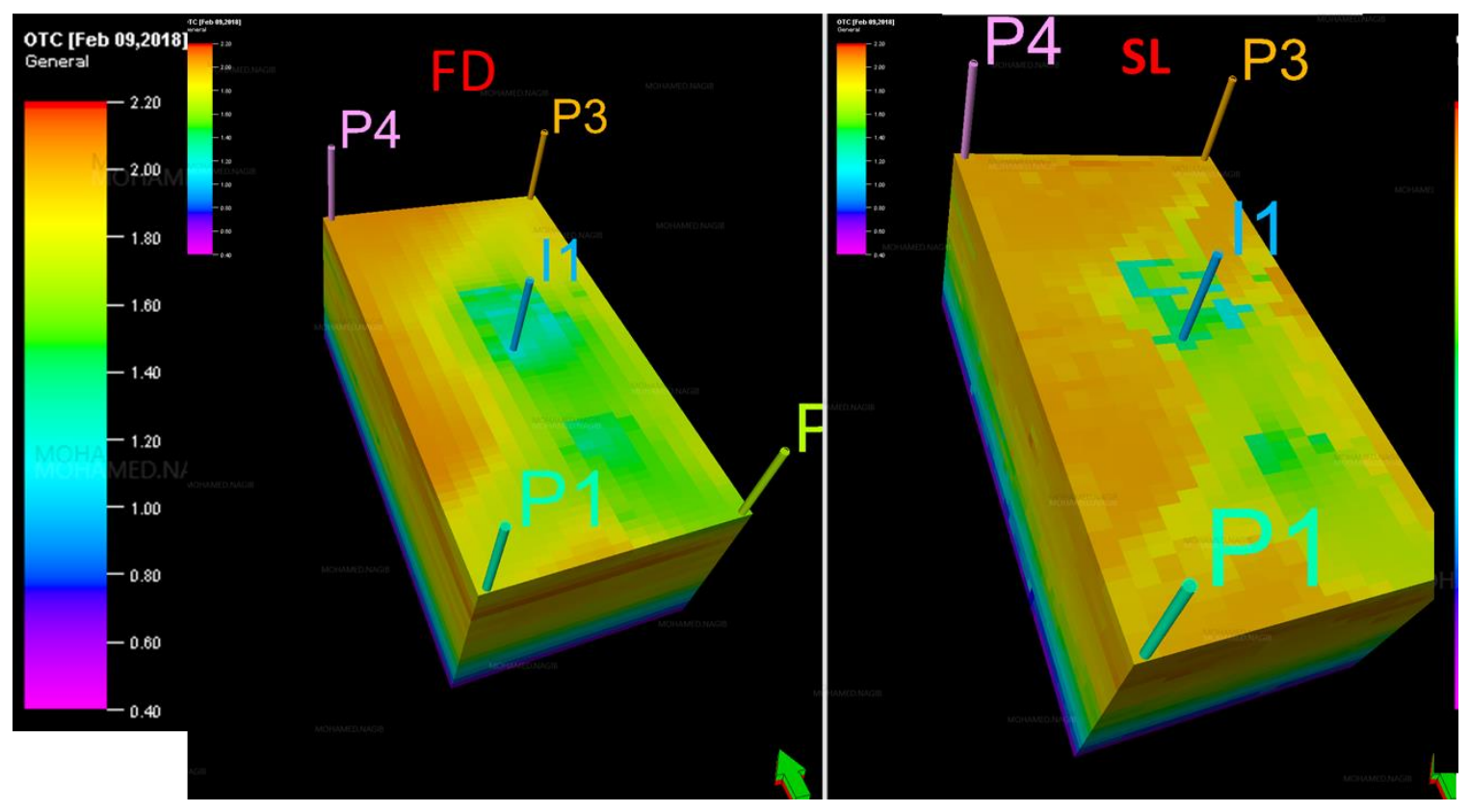

Figure 6-27: OTC of both FD and SL Models.

\subsection{Additional Sensitivities}

In this section, additional sensitivities were performed for gas injection model used on this project. These sensitivities cover the following:

- Depletion case (No Gas Injection)

- Different Gas Injection rates

- Different Bottom Hole Pressures.

\subsubsection{Depletion Case (No Gas injection)}

As a unique sensitivity test, a trial case was done in order to assess SL simulation calculation in the depletion case. This has not been done before, as generally SL simulation is used to track the fluid injection into the reservoir. In this sensitivity, the gas injection rate for the gas injector is set to zero to represent the 
no gas injection case. In the following graphs, the evaluation and comparison of the sensitivity are presented.

For the oil production rate, Figure 6-28 presents the oil production rate forecasted in both the FD and SL cases. The black line represents FD simulator. As can be seen in the plots, both FD and SL predicted oil rates honour the same trend. However, the SL simulation predicted a higher rate in comparison to FD simulation. This mismatch is mainly because SL simulation is generally used to track the fluid displacement by another fluid, hence the time of flight will be calculated and in this case there is no fluid pushing the other fluid in the reservoir.

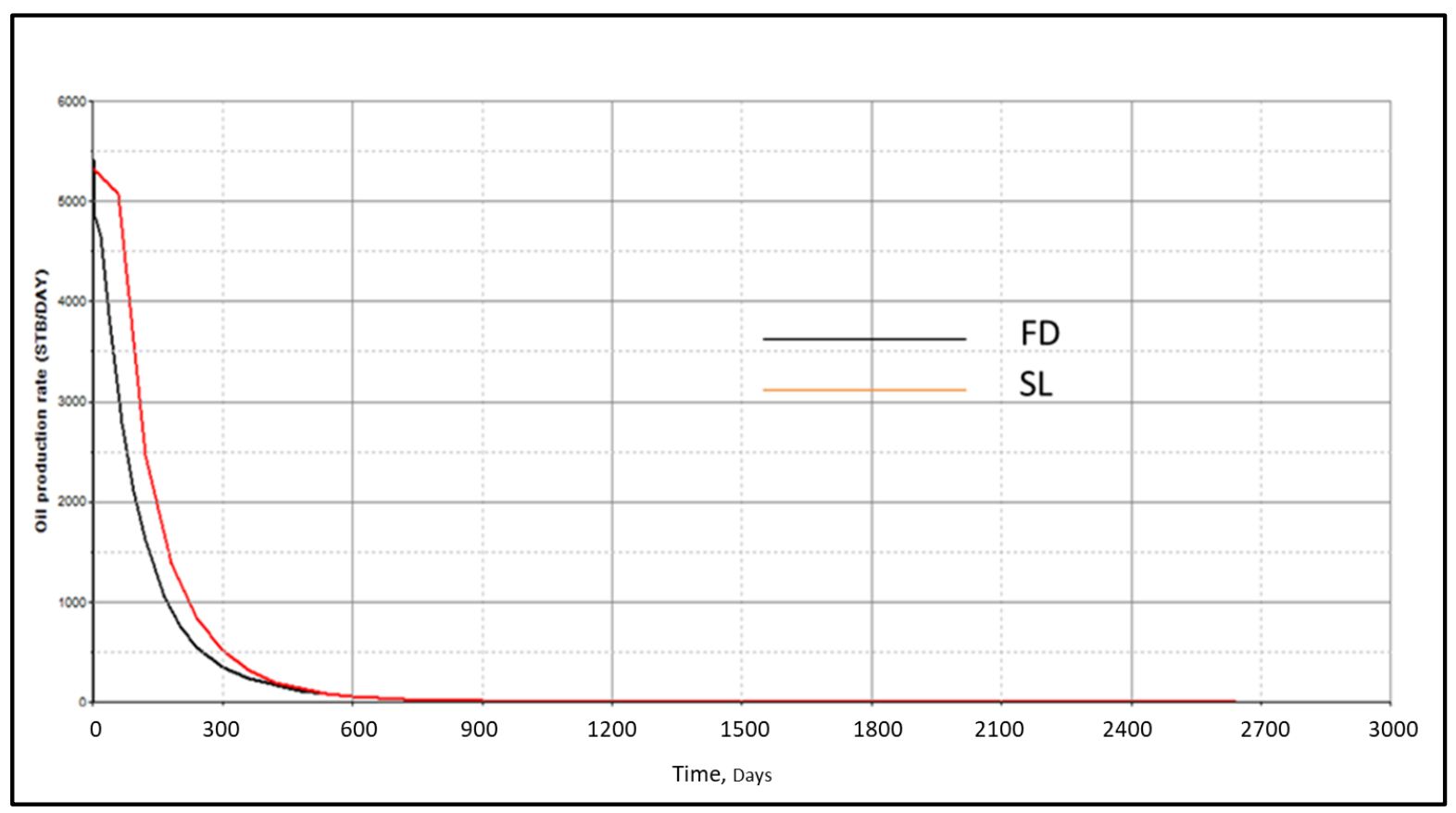

Figure 6-28: Oil production rate for both FD and SL (No Gas).

For cumulative oil production, Figure 6-29 presents cumulative oil production forecasted in both cases, FD and SL. As can be seen from the graph, a general mismatch is obvious as SL is predicting more fluid than FD and this is mainly due to the SL initial rate relatively high in the first timesteps. This mismatch occurs mainly because SL simulation is generally used to track the fluid displacement by another fluid. Hence the time of flight will be calculated and in this case there is no fluid pushing the other fluid in the reservoir. 


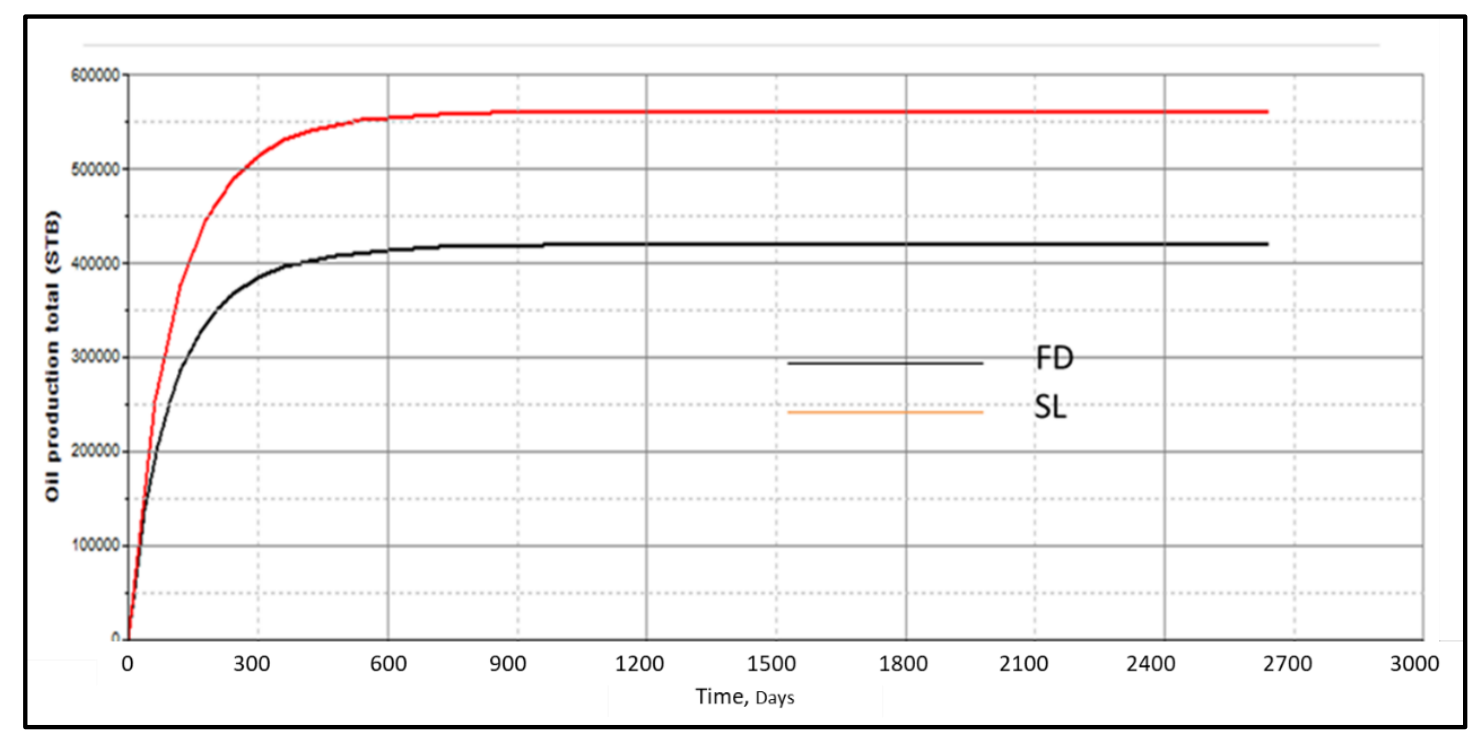

Figure 6-29: Cumulative Oil production rate for both FD and SL (No Gas).

In terms of reservoir pressure performance, Figure 6-30 presents reservoir pressure performance in both cases, FD and SL. As can be seen from the plot, both trends are similar. However, the pressure in the SL simulation is slightly lower in comparison to the FD simulation, but this is not reflected in predicted fluid rates.

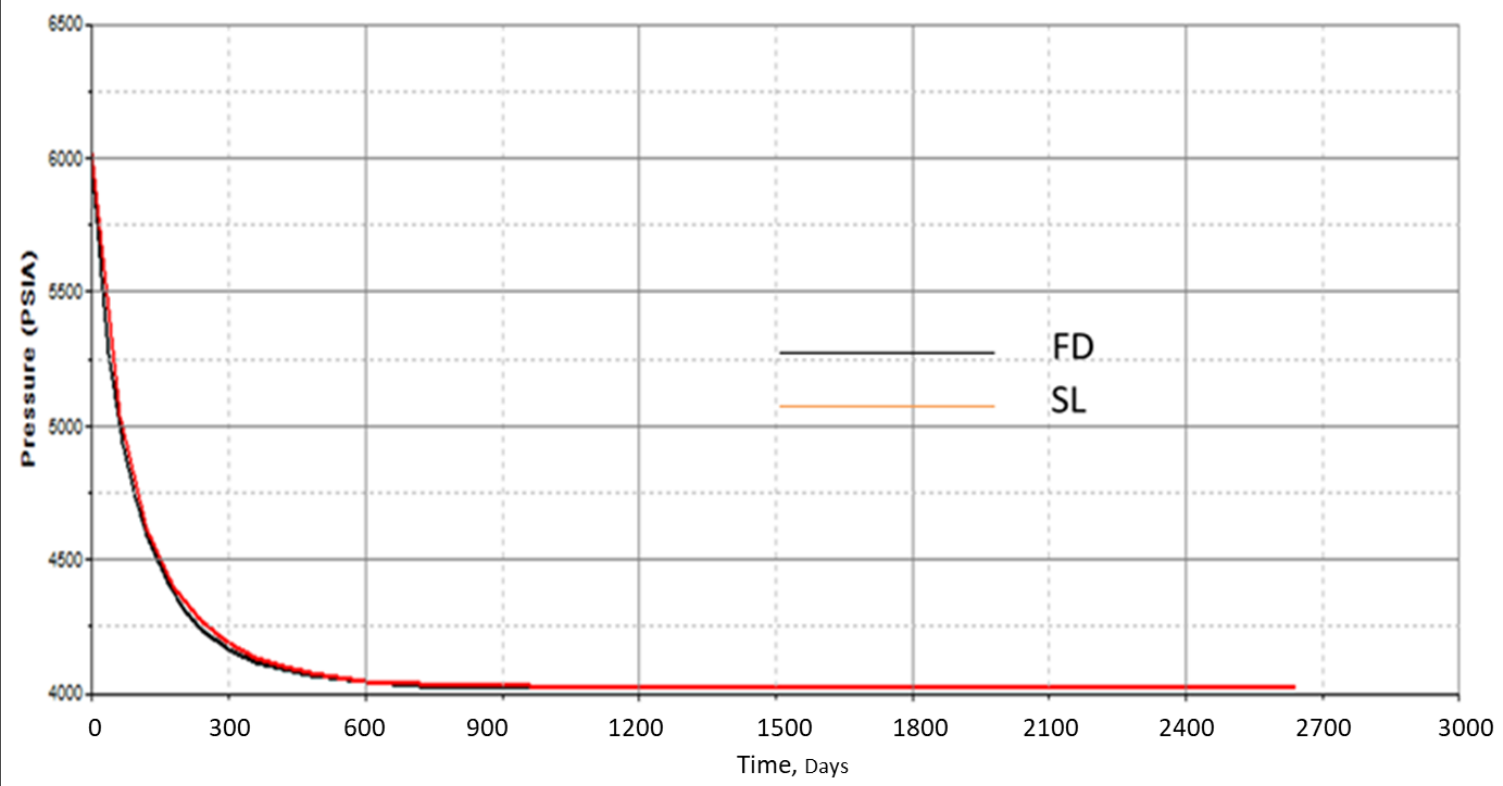


Figure 6-30: Pressure profile for both FD and SL (No Gas).

For total CPU, Figure 6-31 presents the performance in both cases, FD and SL. As can be seen from the plot, TCPU in case of SL is higher than the case of FD.

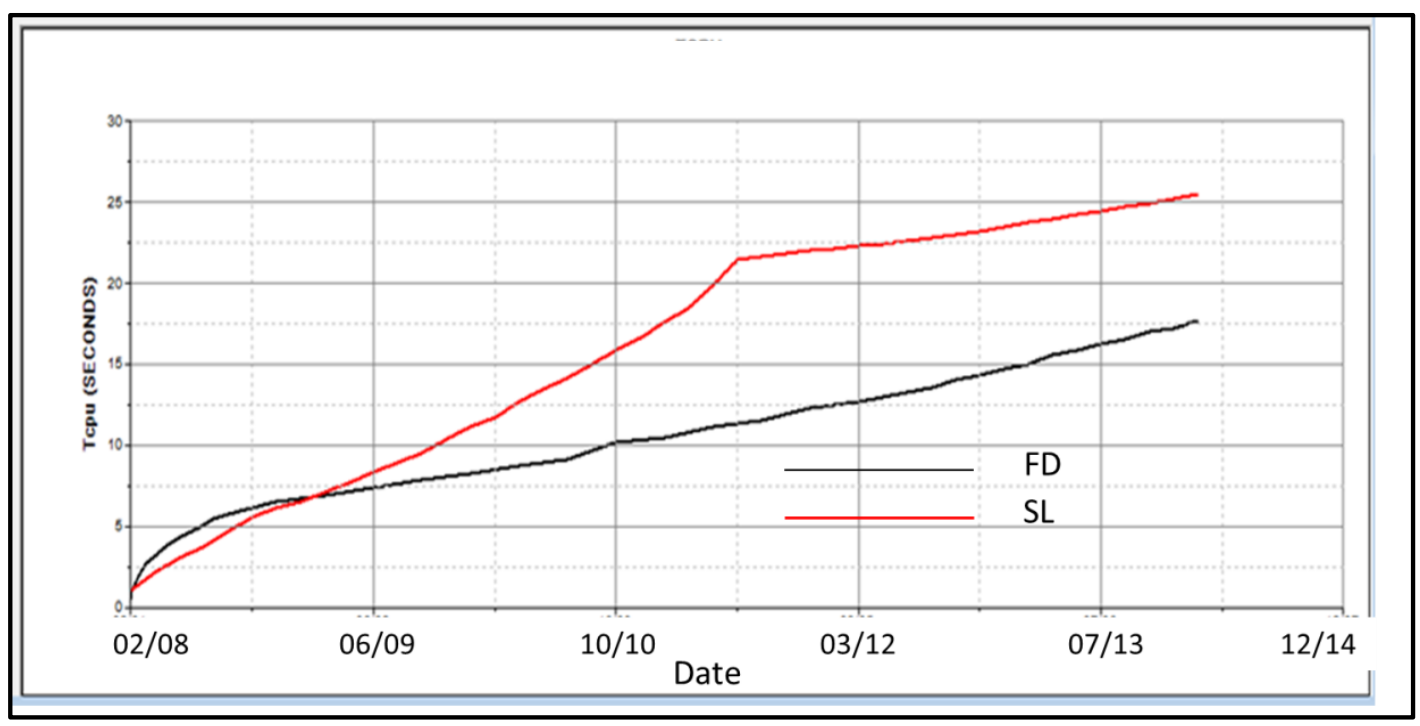

Figure 6-31: Total CPU required for FD and SL.

\subsubsection{Gas Injection Rate Sensitivities}

Additional sensitivities were performed to assess the impact of gas injection rates variations on SL calculations by testing the model on different gas injection rates and monitoring the predicted performance of the model and the stability and the impact on the computational times.

For the oil production rate, Figure 6-32 presents the oil production rate forecasted on different injection rates using FD simulation. As can be seen in the graph, four different gas injection rates were tested and these rates are: 3 million standard cubic feet per day (MM scf/day), 5, 7 and $10 \mathrm{MMscf} / \mathrm{d}$. 


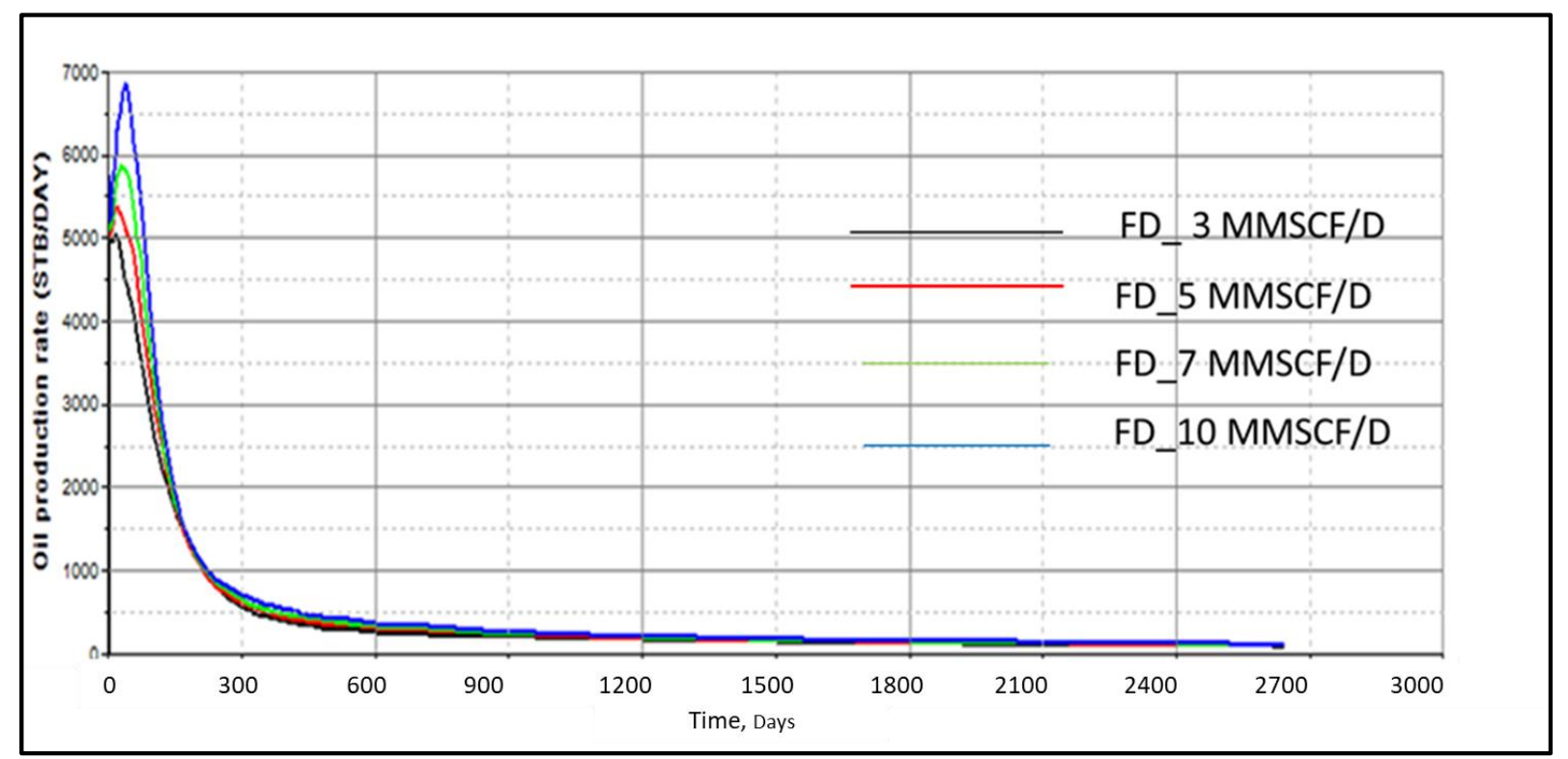

Figure 6-32: Impact of different gas injection rates on Oil production rate for FD simulation.

Figure 6-33 presents the same for SL simulation for the four different gas injection rates. Both models show similar trends in predicting oil rates, with the high gas injection rate reflecting high oil production.

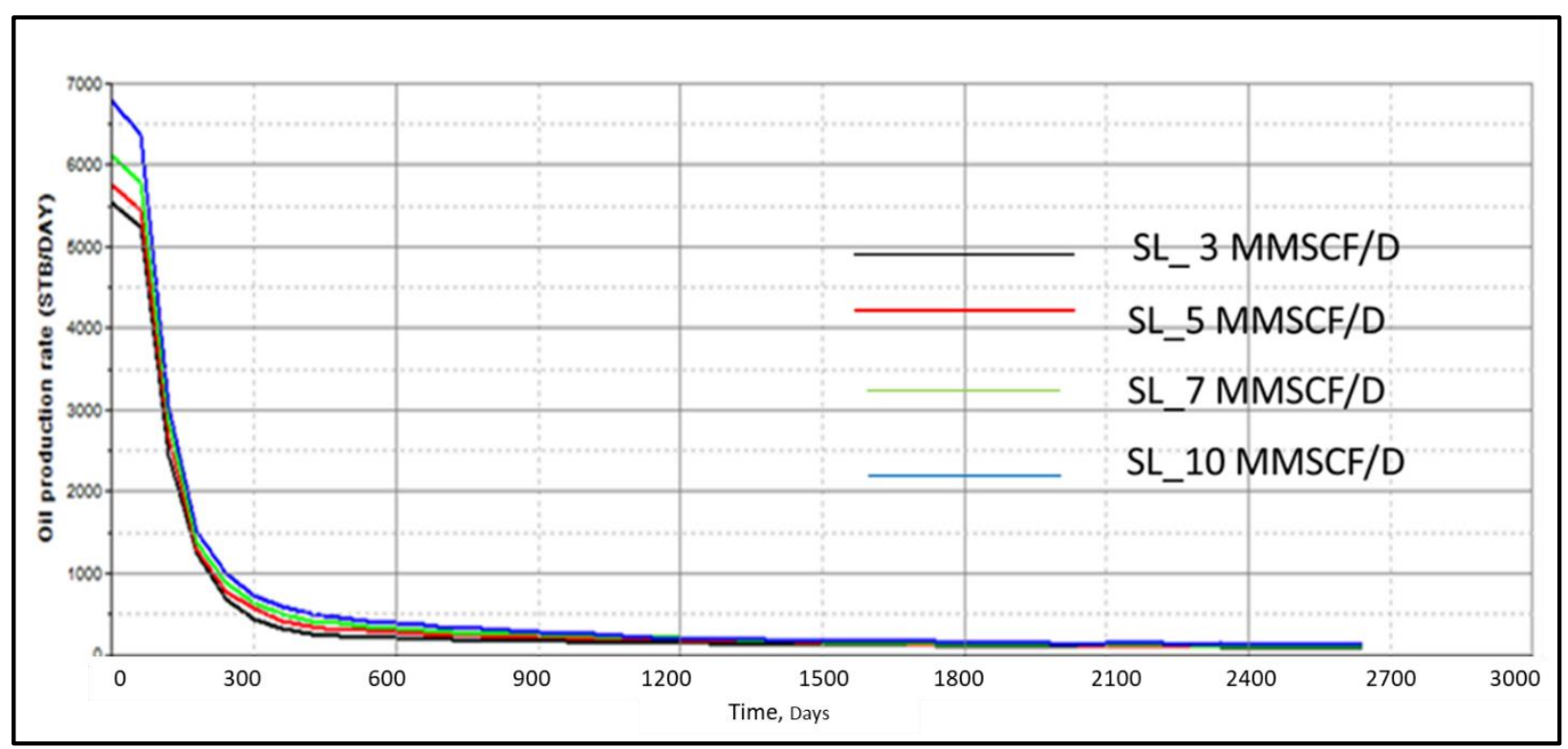

Figure 6-33: Impact of different gas injection rates on oil production rate for SL simulation. 
For cumulative oil production, Figure 6-34 presents cumulative oil production forecasted on different injection rates using FD simulation.

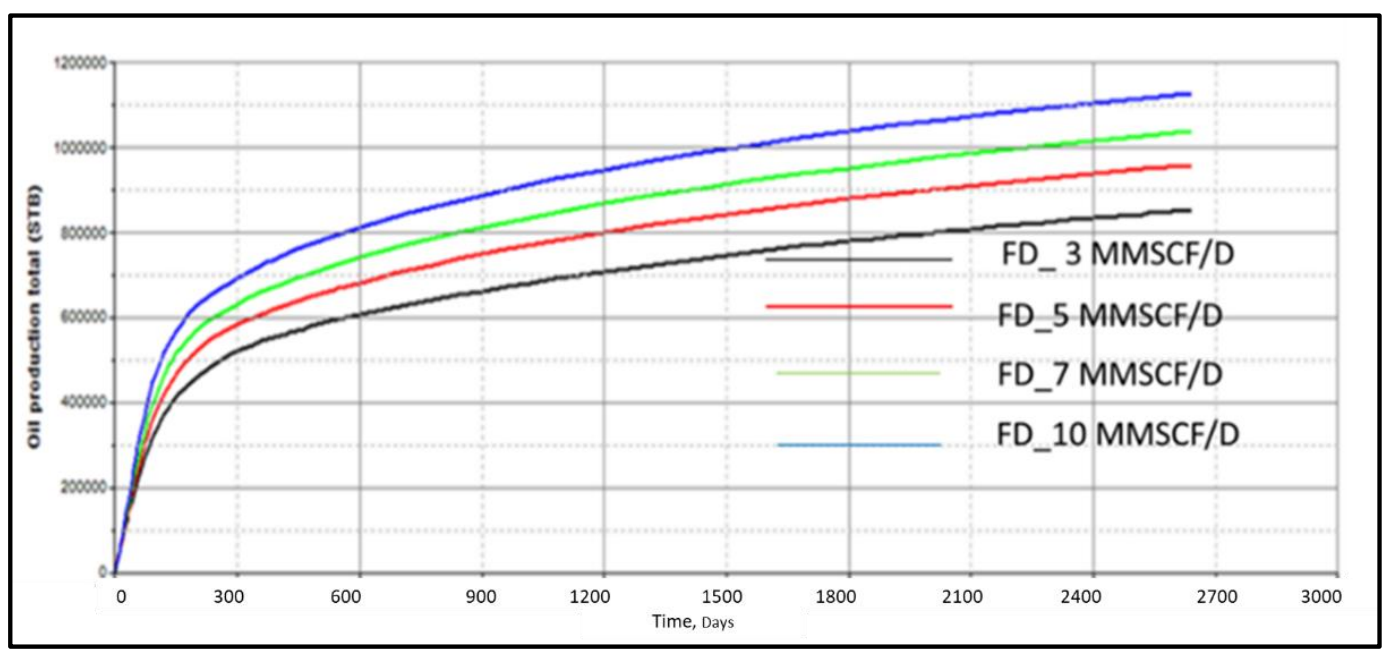

Figure 6-34: Impact of different gas injection rates on Cumulative Oil production for FD simulation.

Figure 6-35 presents the same for SL simulation. Same observations as noticed earlier for oil production rates show that both models are in good agreement.

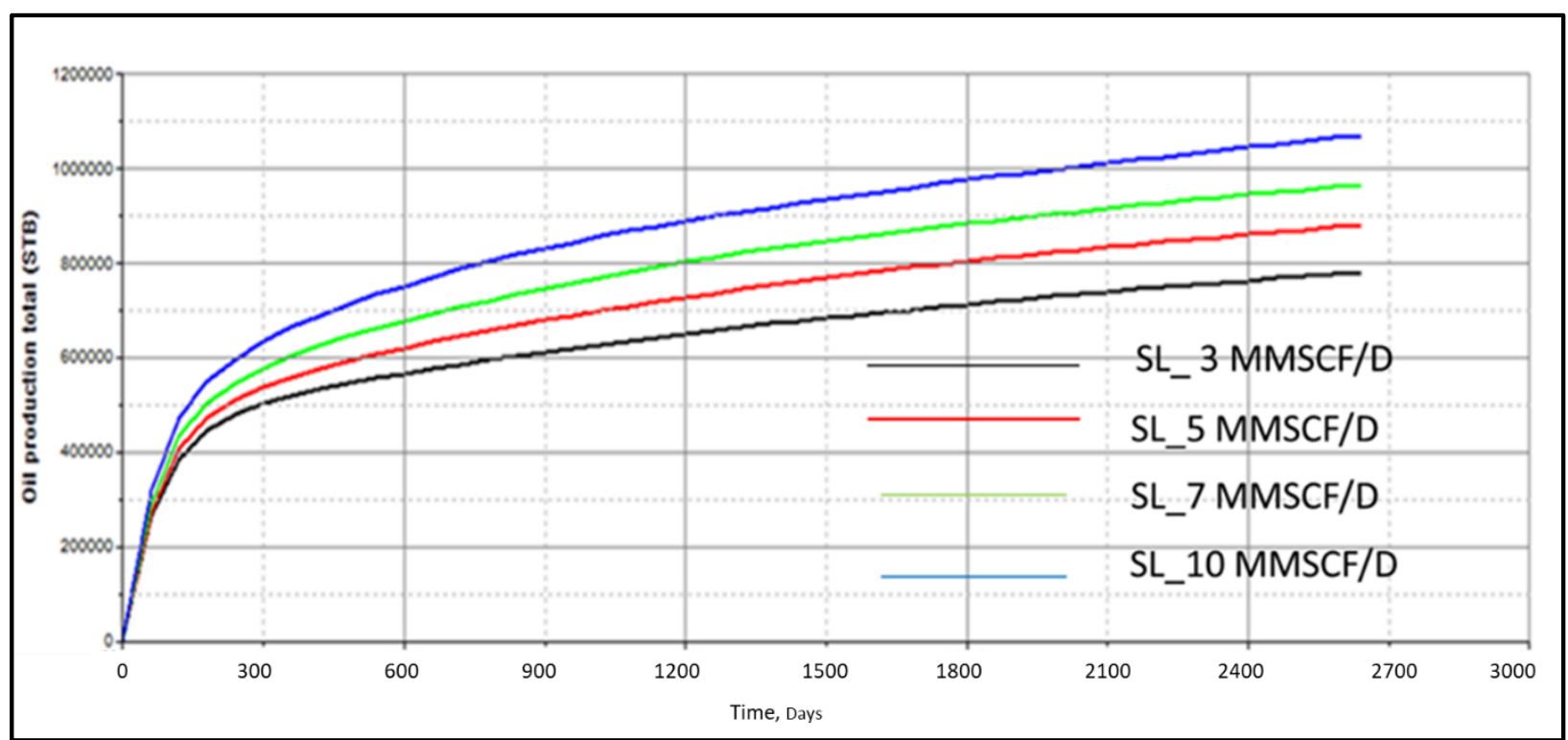

Figure 6-35: Impact of different gas injection rates on Cumulative Oil production for SL simulation.

Figure 6-36 presents the gas production rate forecasted on different injection rates using FD simulation. 


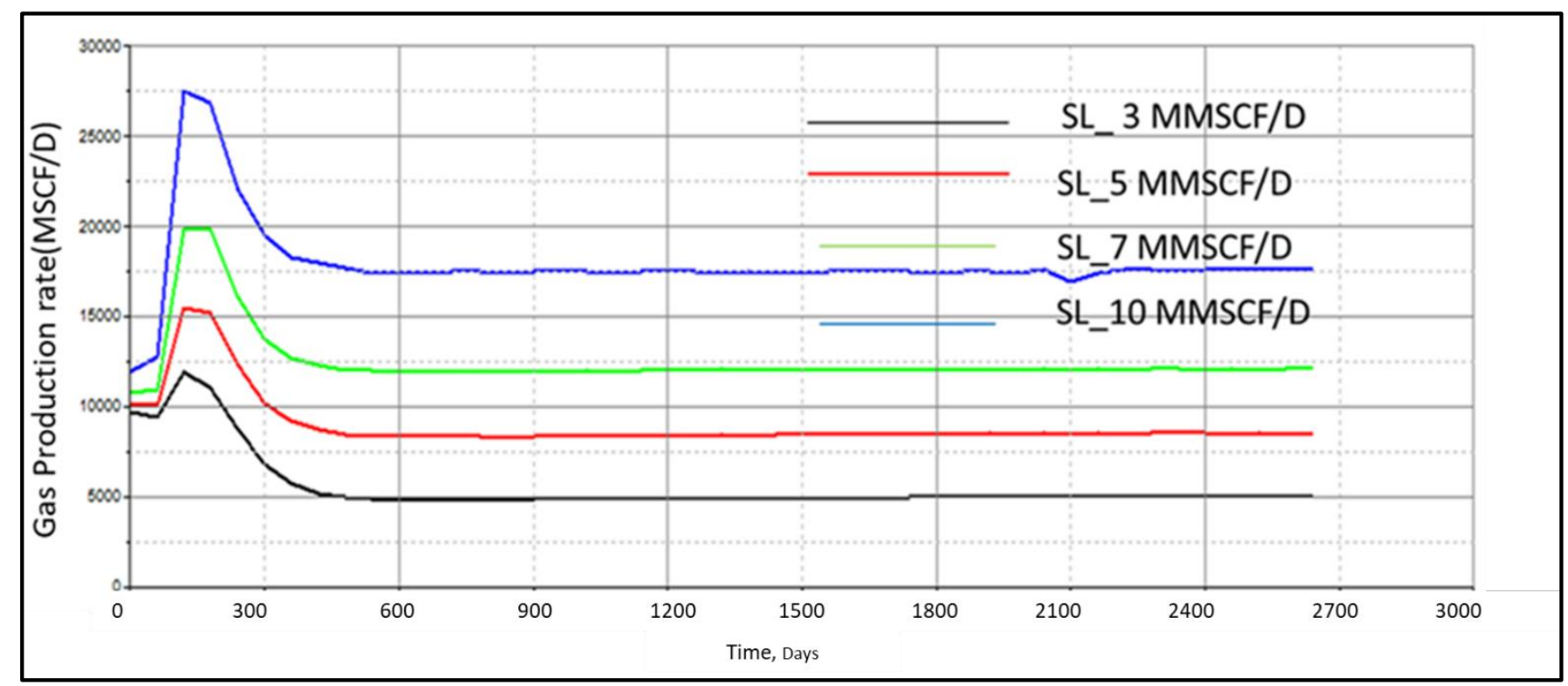

Figure 6-36: Impact of different gas injection rates on Gas Production Rate Performance for FD simulation.

Figure 6-37 presents the gas production rate forecasted on different injection rates using SL simulation.

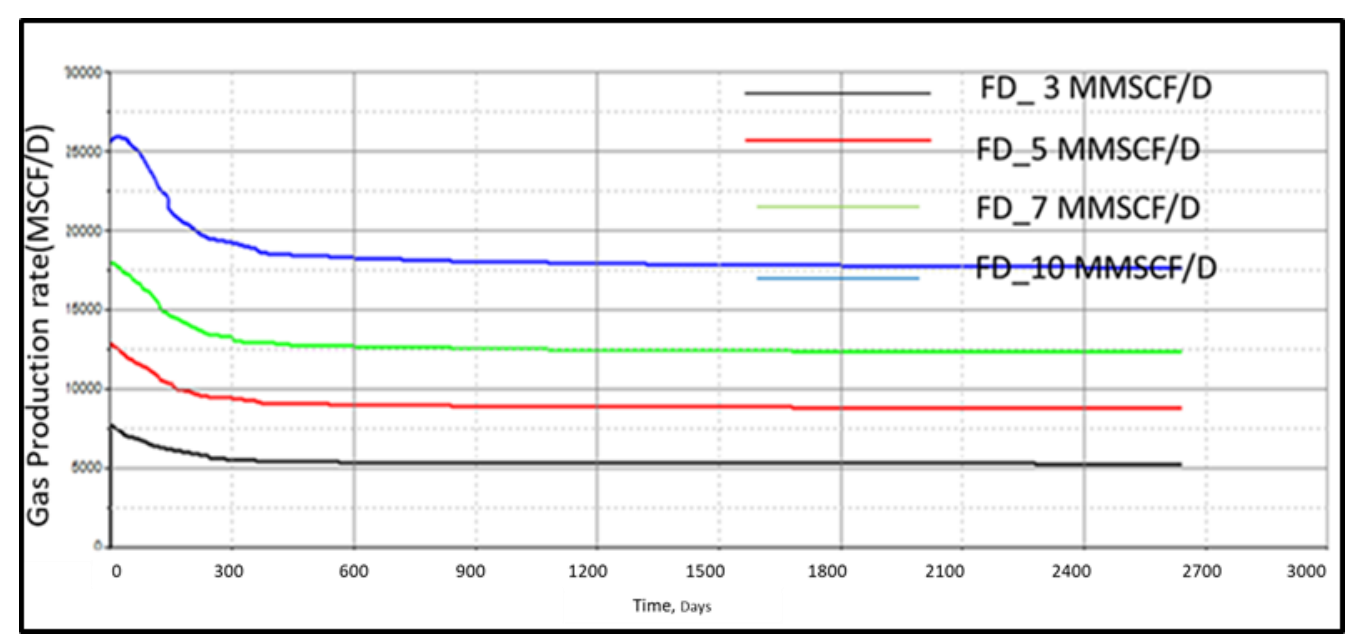

Figure 6-37: Impact of different gas injection rates on Gas Production Rate Performance for SL simulation. 
With regards to bottom-hole pressure, Figure 6-38 present the impact of gas injection rates on bottom hole pressure for FD model. As can be seen, lower injection pressure directly impacting bottom hole pressure.

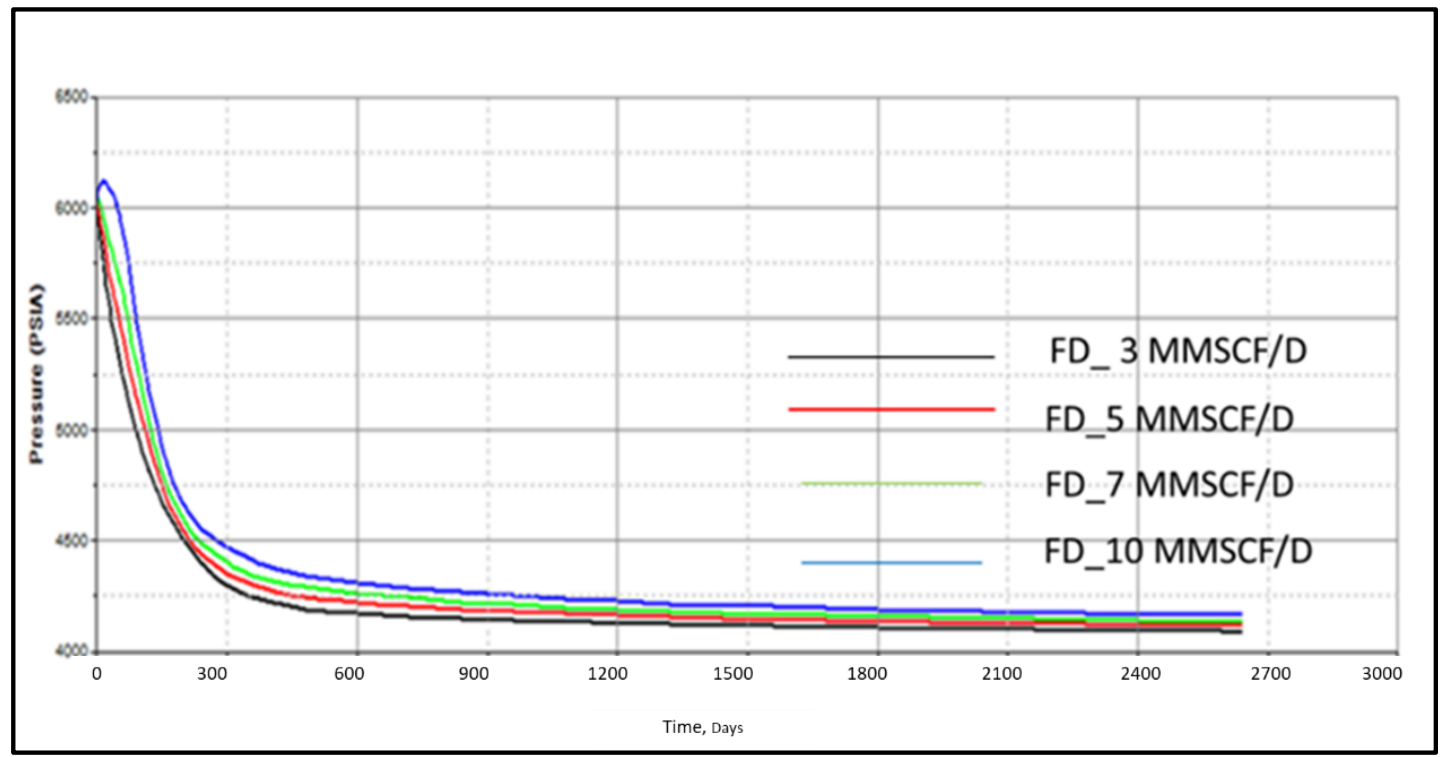

Figure 6-38: Impact of different gas injection rates on Reservoir Pressure Performance for FD simulation.

For the SL model, For the SL model, Figure 6-39 presents the impact of gas injection rates on bottom hole pressure. Similar trend is observed when compared to FD model. Injection rates directly affecting the reservoir pressure. Higher injection rate results higher bottom hole pressure.

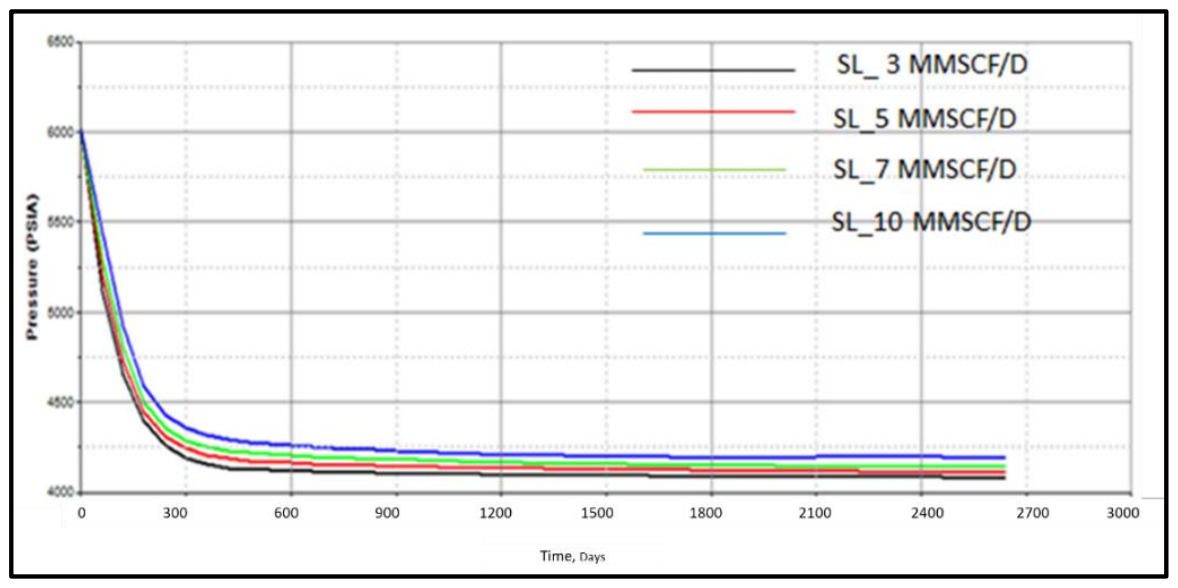


Figure 6-39: Impact of different gas injection rates on Reservoir Pressure Performance for SL simulation.

\subsubsection{Bottom-hole Pressure Sensitivities}

The model was also tested for different bottom hole pressures in order to assess the impact of various bottom pressures on the predicted performance and model stability.

For the oil production rate, Figure 6-40 presents the oil production rate forecasted on different bottom hole pressures using FD simulation. The results show that once bottom hole pressure drops inside the reservoir, SL is not able to continue fluid flow inside the model. On these sensitivities, at bottom-hole pressure of 3000psi, the SL model cannot sustain and wells will stop producing.

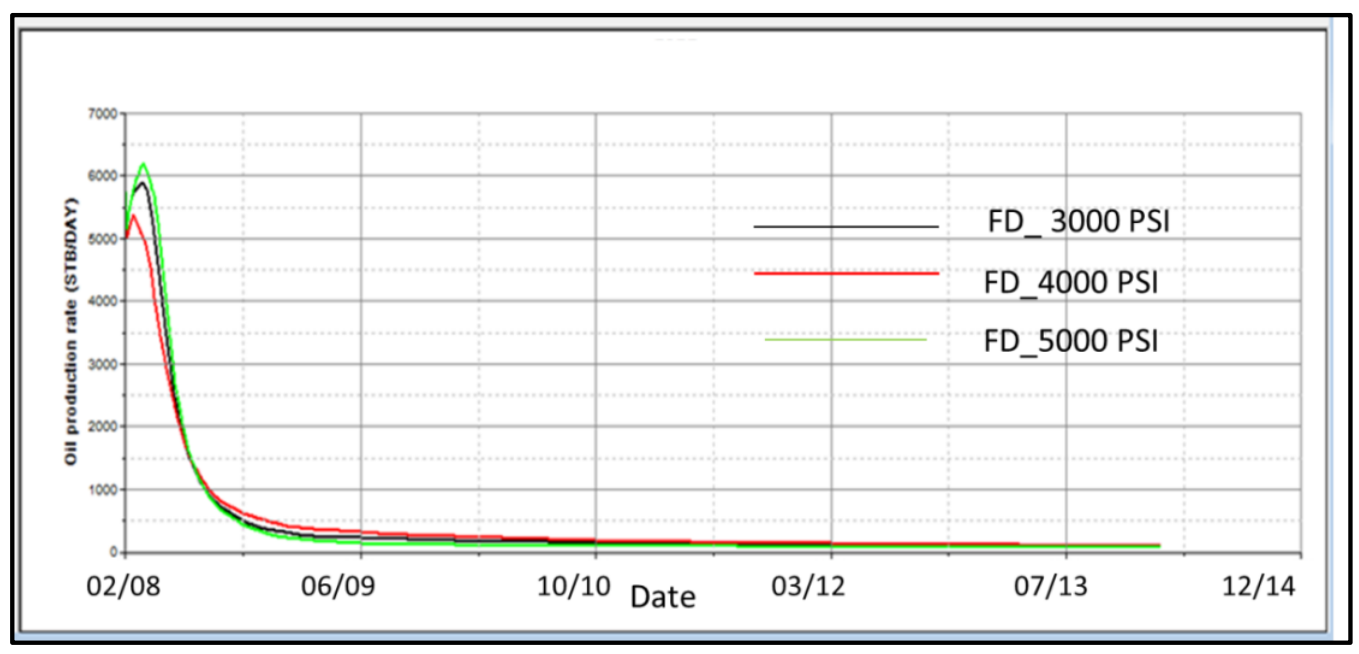

Figure 6-40: Impact of different Bottom-hole pressures on oil production rate for FD simulation.

For the oil production rate, Figure 6-41 presents the oil production rate forecasted on different bottom hole pressures using SL simulation. The results show that once bottom hole pressure drops inside the reservoir, SL is not able to continue fluid flow inside the model. On these sensitivities, at bottom-hole pressure of 3000psi, the SL model cannot sustain and wells will stop producing. 


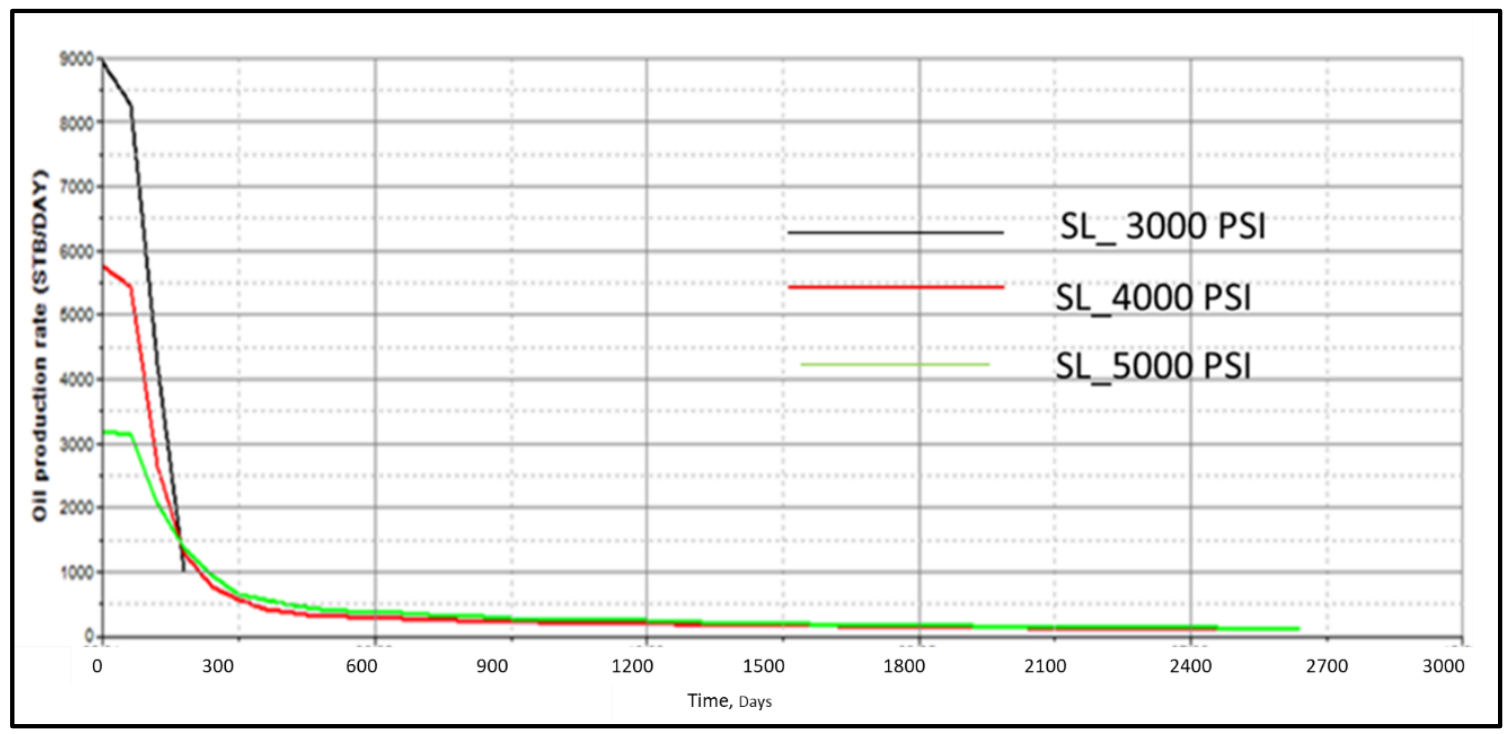

Figure 6-41: Impact of different Bottom-hole pressures on oil production rate for SL simulation.

For cumulative oil production, Figure 6-42 presents cumulative oil production forecasted on different bottom-hole pressures using FD simulation.

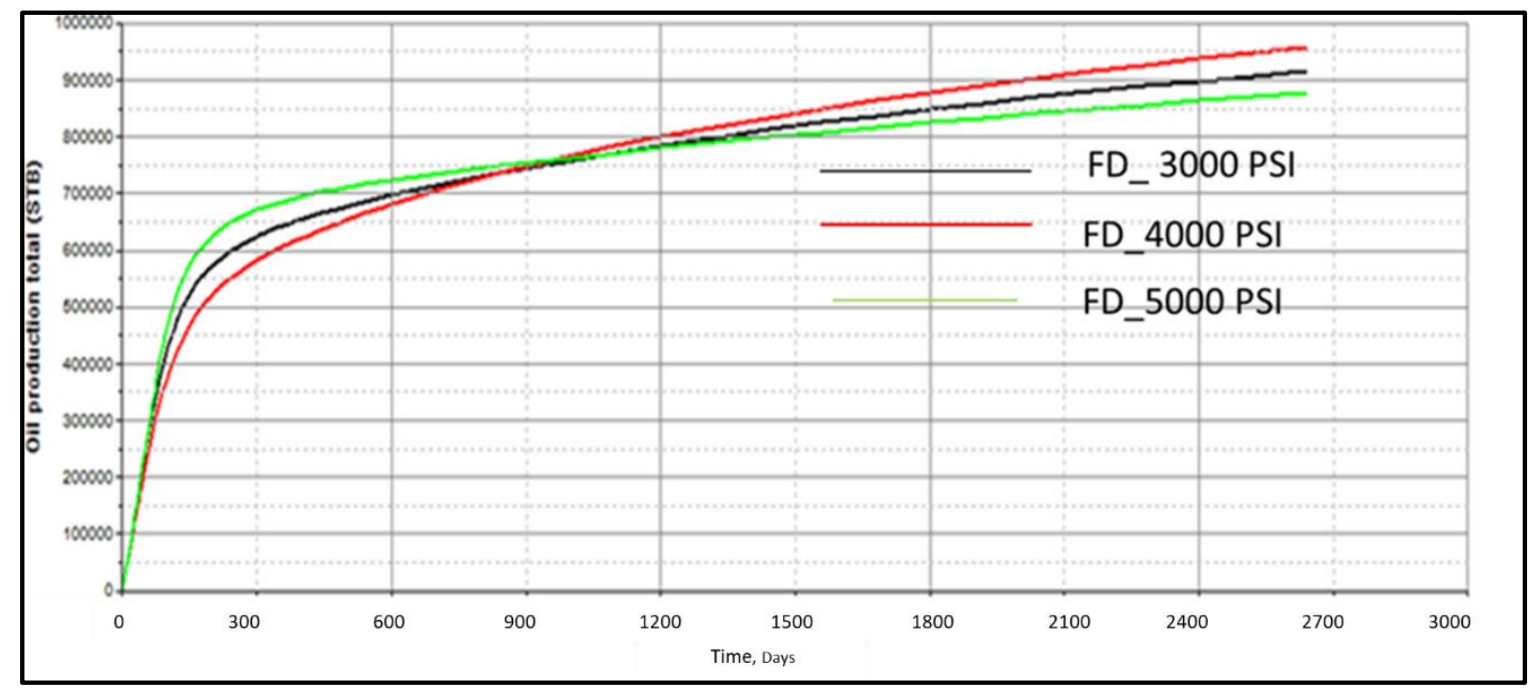

Figure 6-42: Impact of different bottom-hole pressures on cumulative oil production for FD simulation.

Figure 6-43 presents cumulative oil production forecasted on different bottom-hole pressures using SL simulation. 


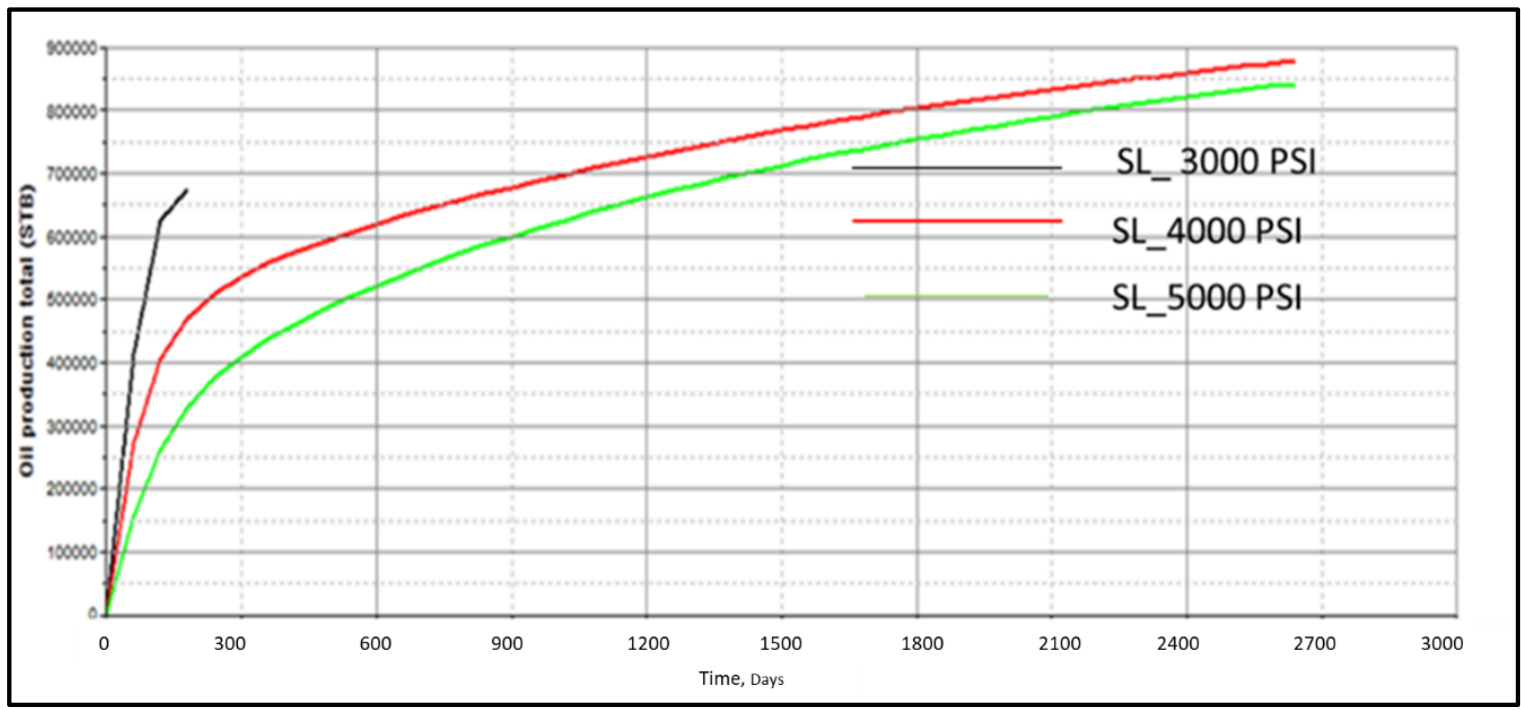

Figure 6-43: Impact of Bottom-hole pressures on Cumulative Oil production for SL simulation.

Figure 6-44 presents the gas production rate forecasted on bottom hole pressures using FD simulation.

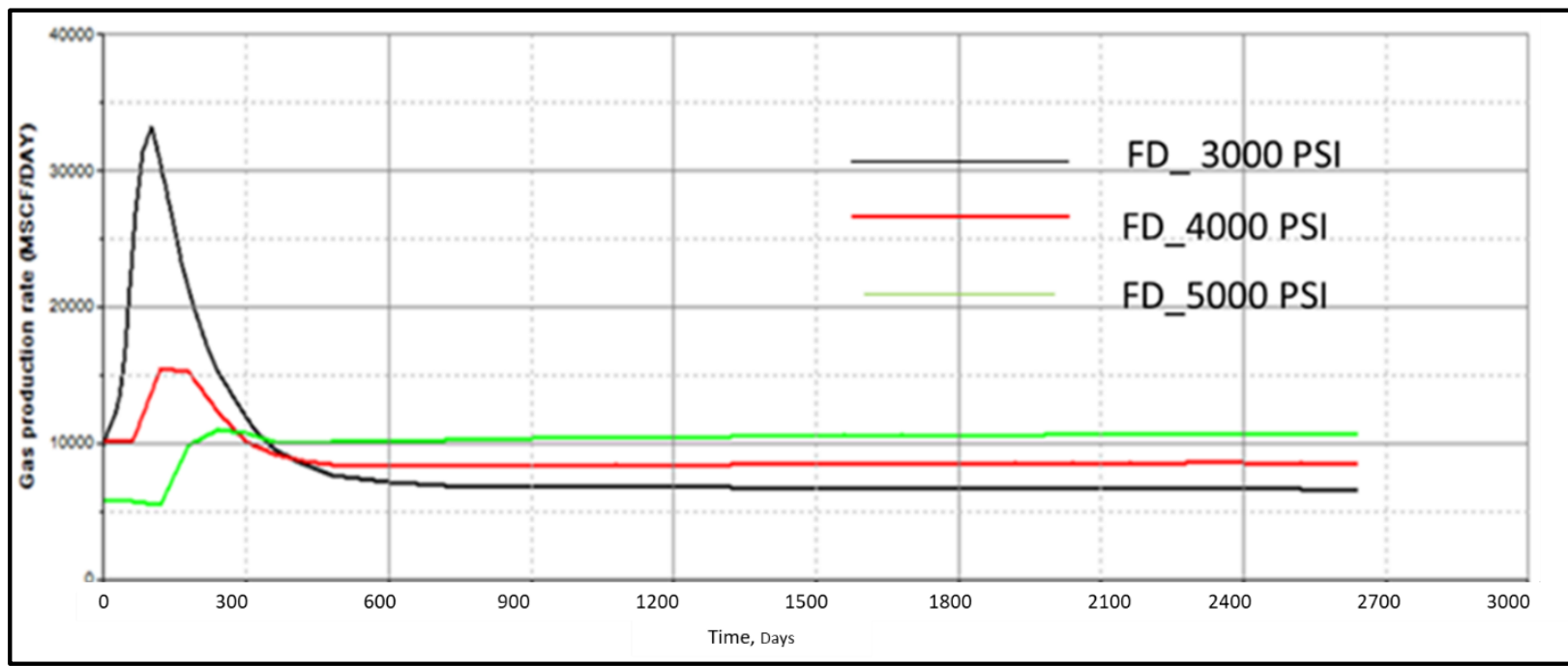

Figure 6-44: Impact of Different Bottom-hole Pressures on Gas Production Rate Performance for FD simulation.

Figure 6-45 presents the gas production rate forecasted on bottom hole pressures using SL simulation. 


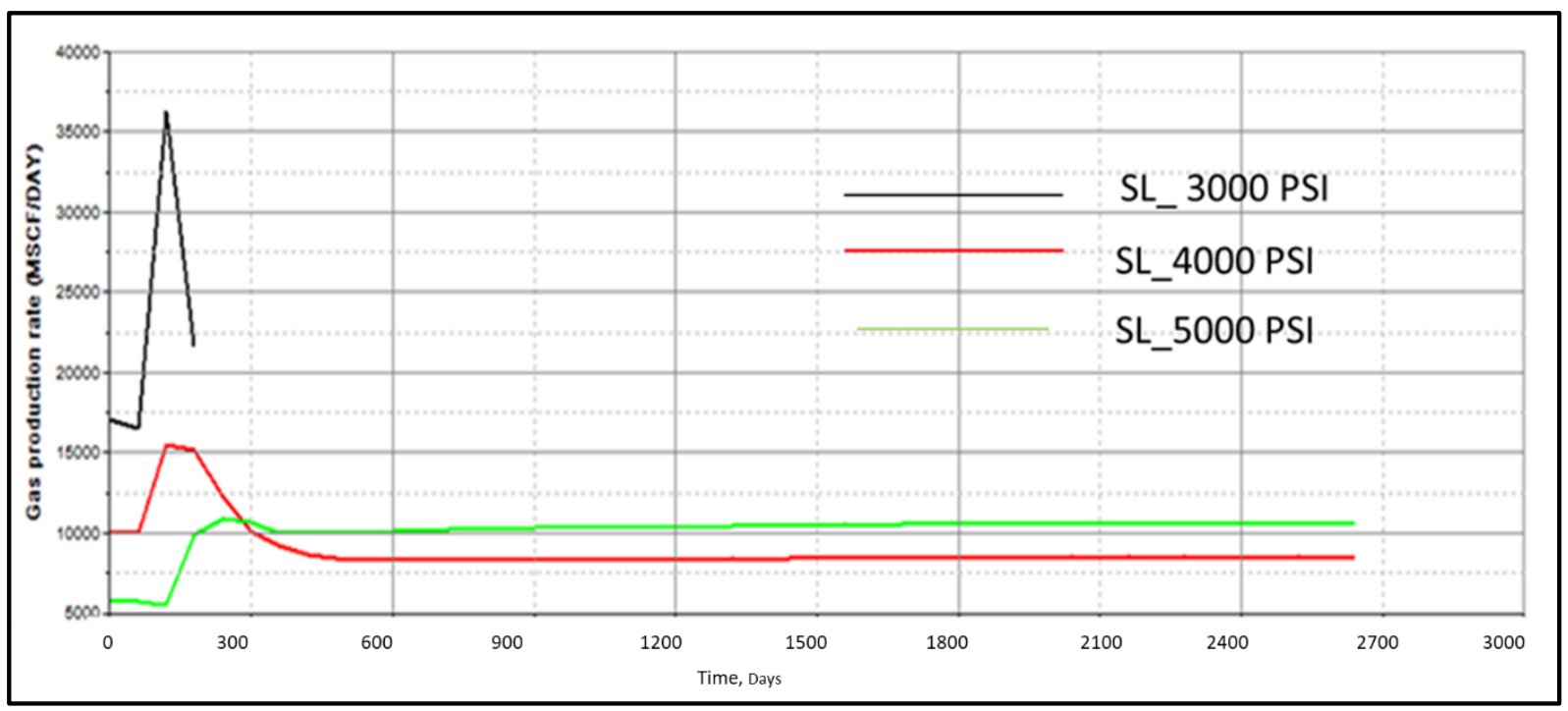

Figure 6-45: Impact of Different Bottom-hole Pressures on Gas Production Rate Performance for SL simulation.

Figure 6-46 presents the impact of bottom hole pressure on gas injection rates for FD model

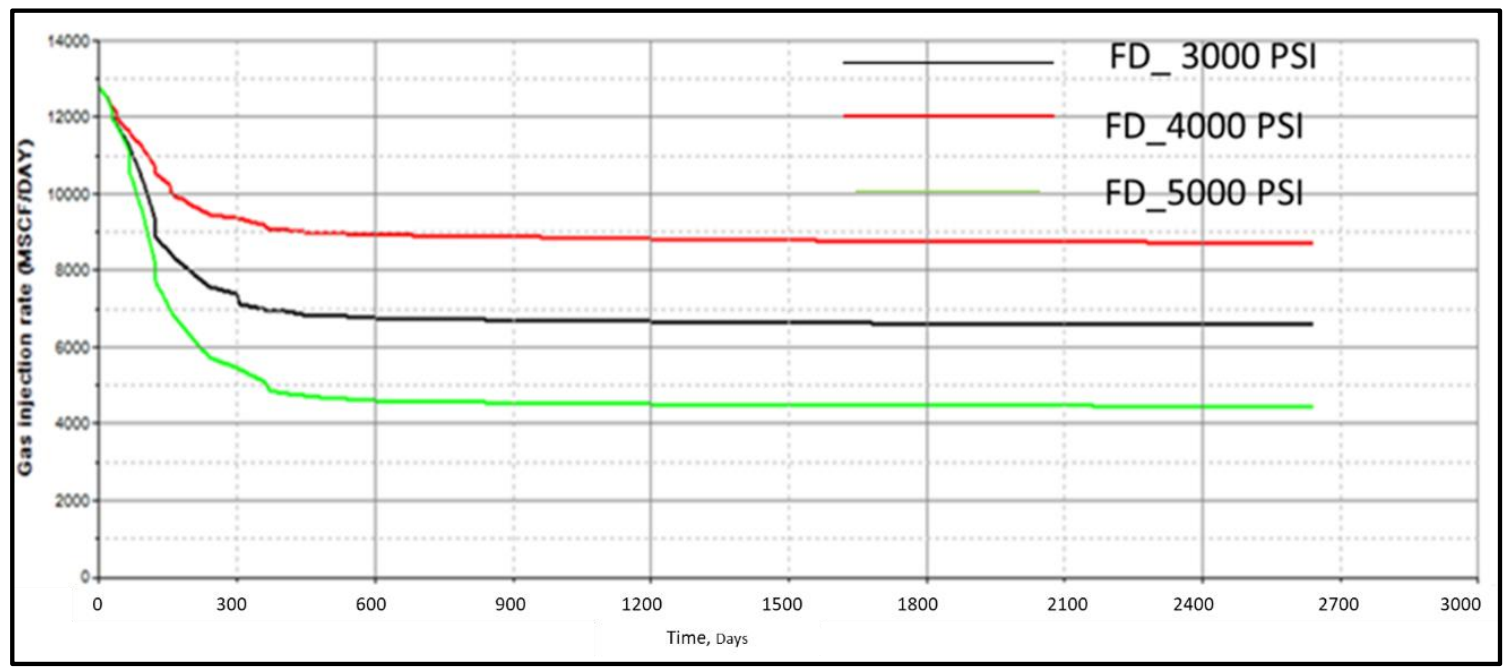

Figure 6-46: Impact of Different Bottom-hole Pressures on Gas Injections Rates for FD simulation.

For SL Model, Figure 6-47 presents the impact of bottom hole pressure on gas injection rates. 


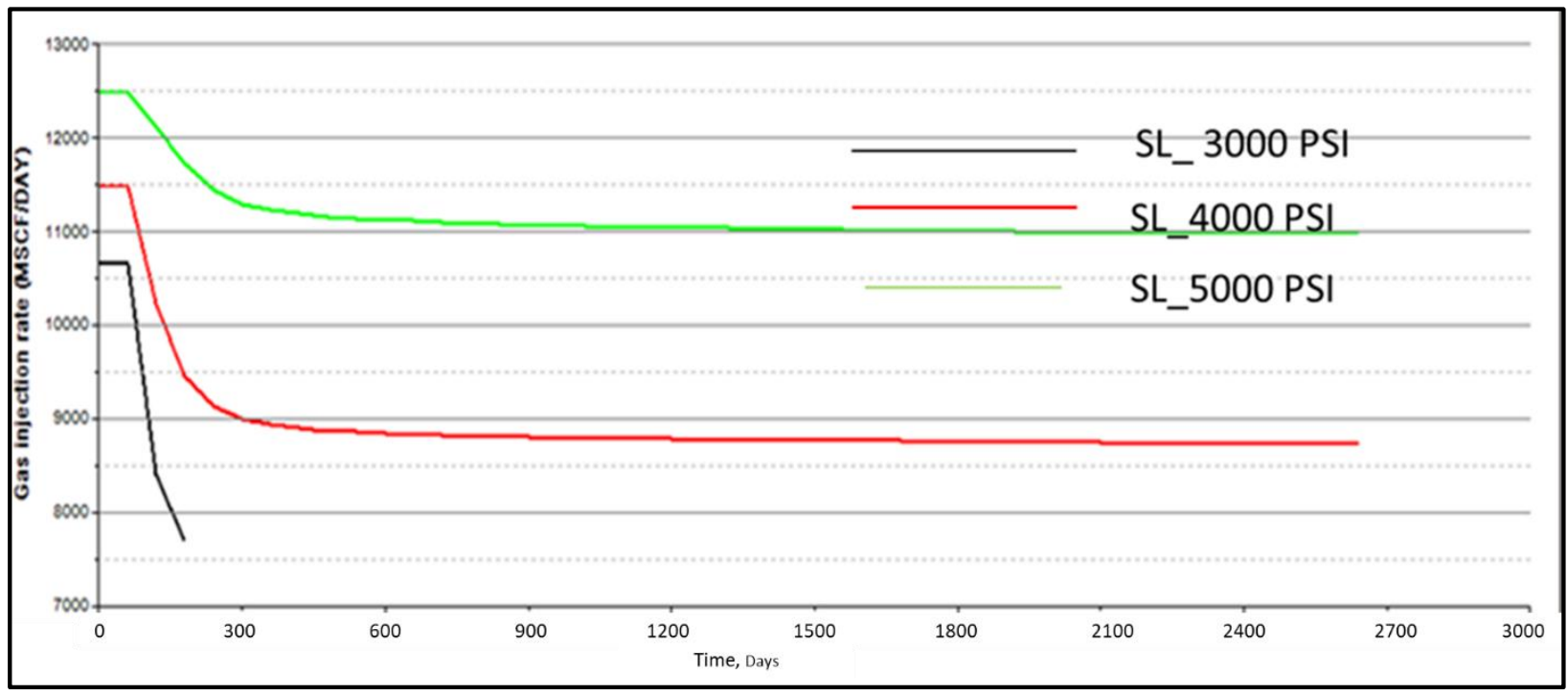

Figure 6-47: Impact of Different Bottom hole Pressures on Gas Injection Rates for SL simulation.

For the reservoir pressure, Figure 6-48 presents the reservoir pressure forecasted at different bottom hole pressures using FD simulation.

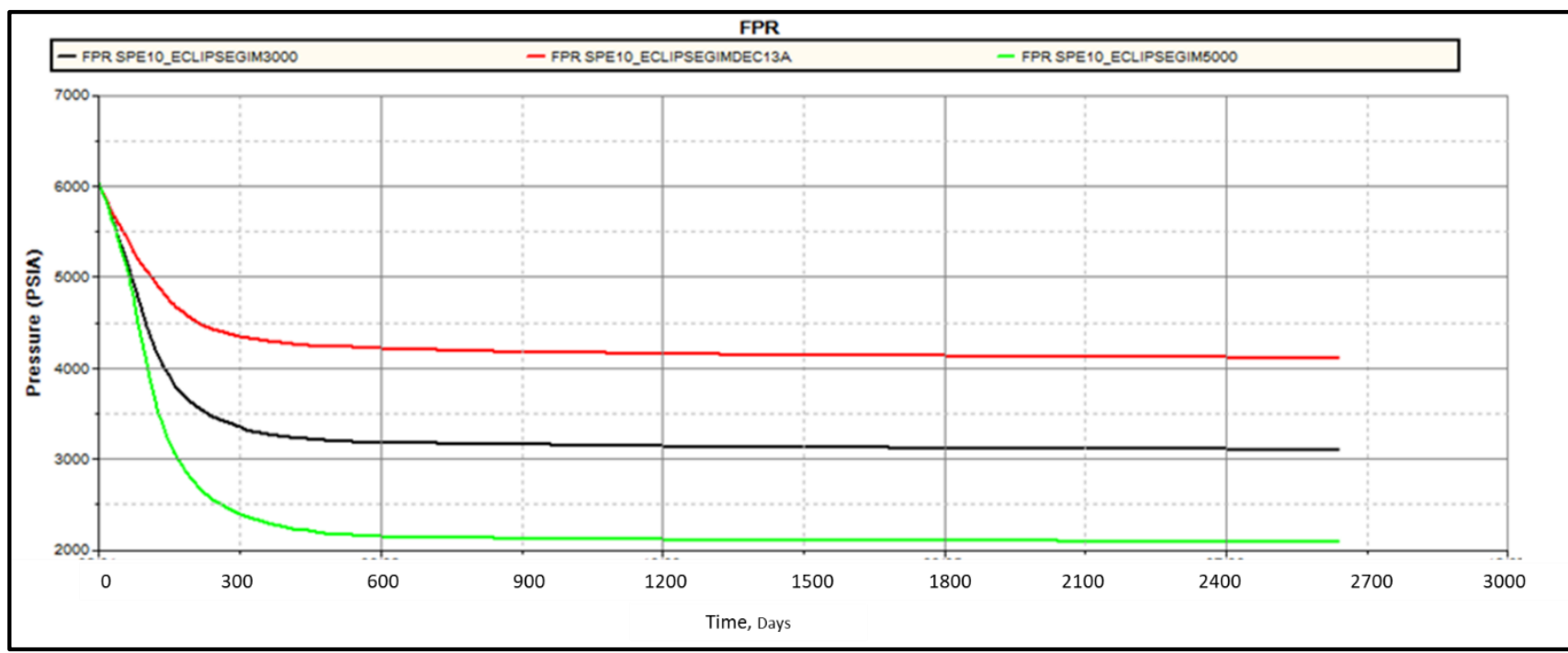

Figure 6-48: Impact of Different Bottom-hole Pressures on Reservoir Pressure Performance for FD simulation. 
Figure 6-49 presents the reservoir pressure forecasted at different bottom hole pressures using SL simulation.

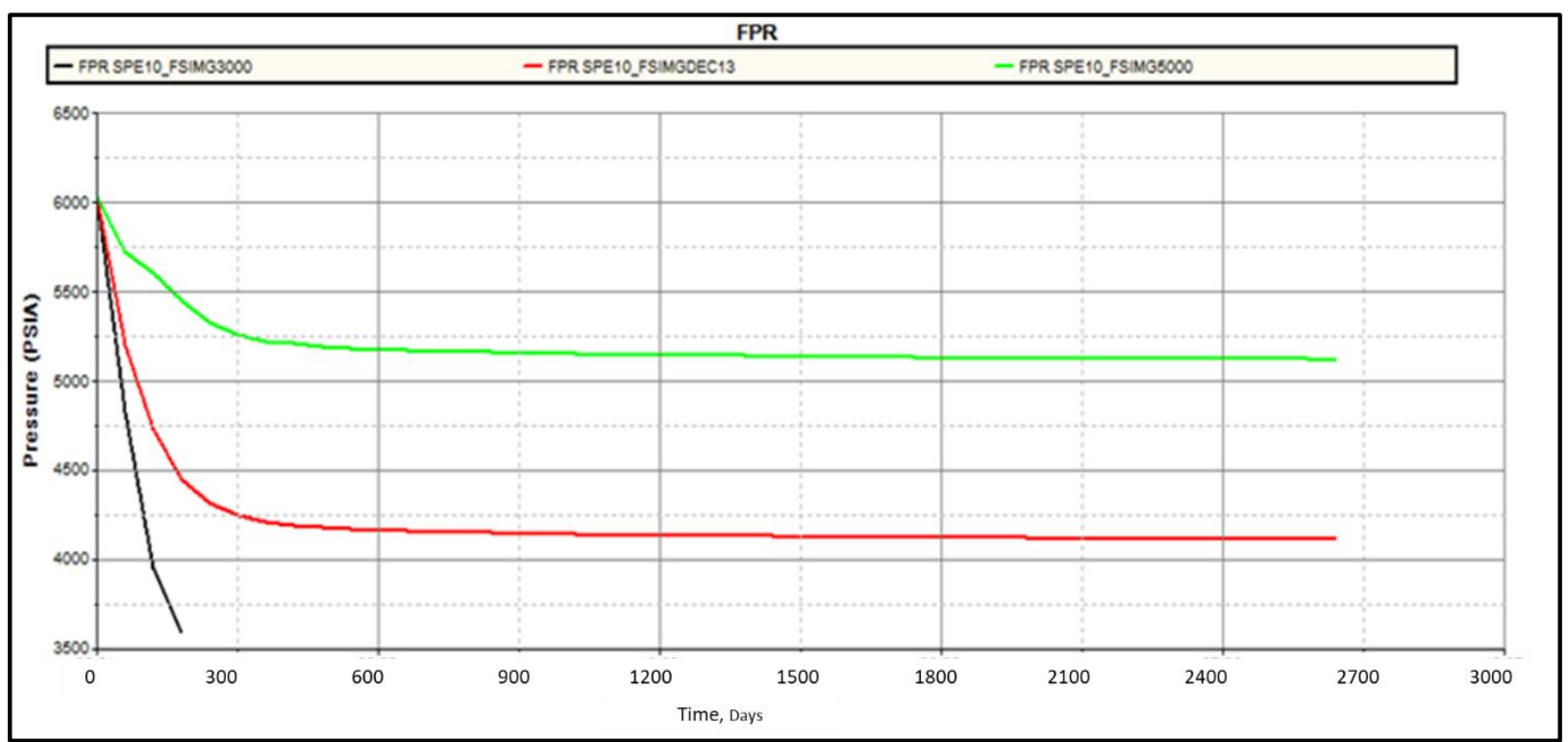

Figure 6-49: Impact of different bottom-hole pressures on Reservoir Pressure Performance for SL simulation.

\subsection{Conclusions:}

- Streamline simulation for gas injection processes generally decomposes the problem into a $3 \mathrm{D}$ pressure solve which is used to determine the streamlines and set $1 \mathrm{D}$ calculations for these streamlines that represent the fluid displaced.

- As a result of this streamline, methods are generally fast when the flow is dominated by the heterogeneity of the rocks as the streamlines slowly change in time, allowing larger time steps relative to the conventional FD technique.

- The base line gas injection case is presented after using the live oil relative permeability date, and the SL simulation model is run fully to the end of the simulation period. Tuning options for the saturation solver were used in order to improve the SL simulation model performance and minimise the relative error between FD and SL simulations. All well control parameters used in both FD and SL simulation models are the same to ensure of the consistency between both models and minimise the relative error. 
- The results showed that the initial runs without the tuning options of the saturation solver showed an extremely high relative error in comparison to the latest run results.

- With the less relative error achieved from this work, SL simulation modelling can be used in gas displacement processes. Base case gas injection models with the same parameters were also used to test and assess the impacts of no gas injection rate, different gas injection rates and different bottom-hole pressures. 


\section{Chapter 7}

\subsection{High Resolution Grid Gas Injection Model}

One of the main advantages of SL simulation over FD simulation is the ability to handle non upscaled geological models (Fine Grid), and the ability to capture all and smaller geological features in a fast manner. Fine scale grid model (high resolution) are presented in this chapter. High resolution model was generated from the base case coarse grid and test the performance of the streamline simulation in high resolution in comparison to that on the coarse grid are presented.

The base case gas injection model was uploaded into the gridding package and the number of grid cells were modified twice in both the $\mathrm{X}$ and $\mathrm{Y}$ directions. Both porosity and permeability were upscaled based on the new fine grid, making sure that all the heterogeneity in the base case model was captured in the fine grid model. The new grid dimensions are 34x110x15 grid blocks in comparison to the original coarse grid $17 \times 55 \times 15$.

Figure 7-1 shows the new grid refinement vs. base case grid and as can be seen, the porosity distribution is still honoured on the new grid.

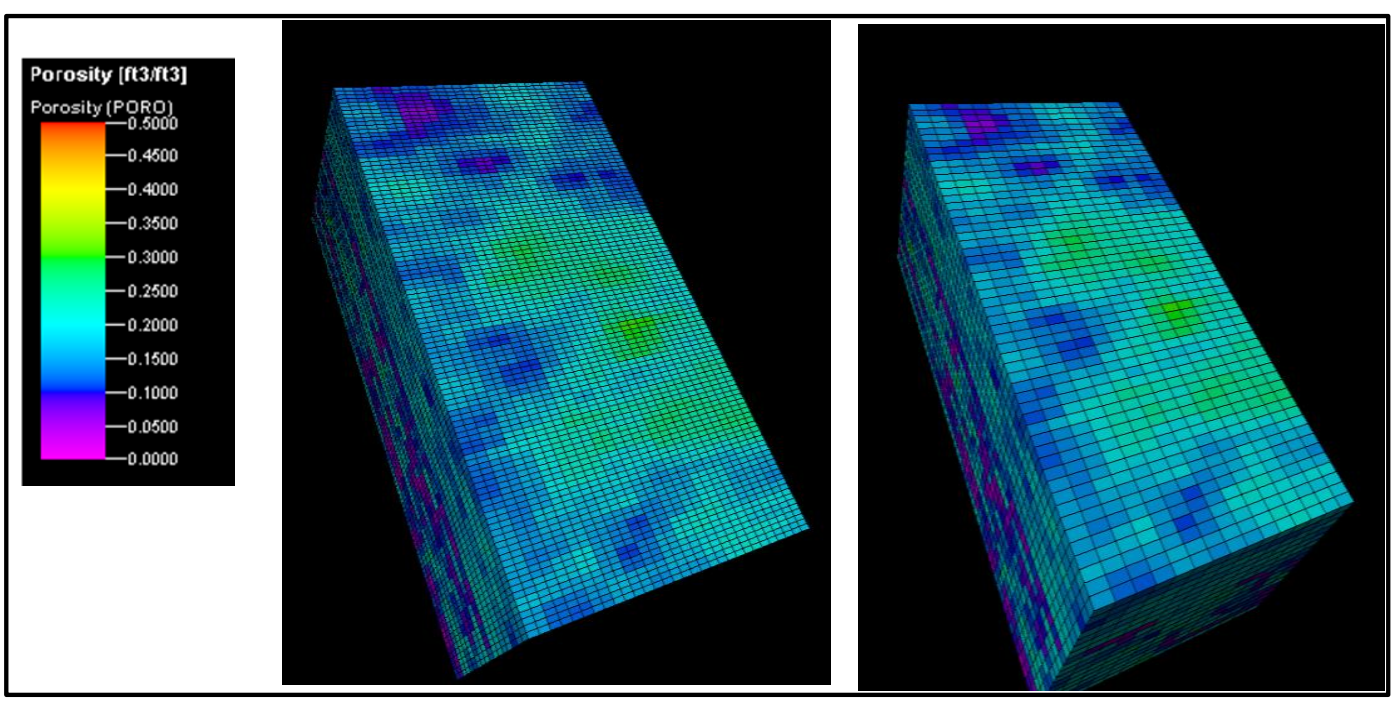

Figure 7-1: High Resolution Porosity Grid vs. Low Resolution 
Figure 7-2 shows cross section for the permeability of the new grid refinement vs. base case grid and as can be seen, the permeability distribution is still honoured on the new grid. Figure 7.3 represents the permeability histogram of the fine grid vs Coarse grid showing no differences.

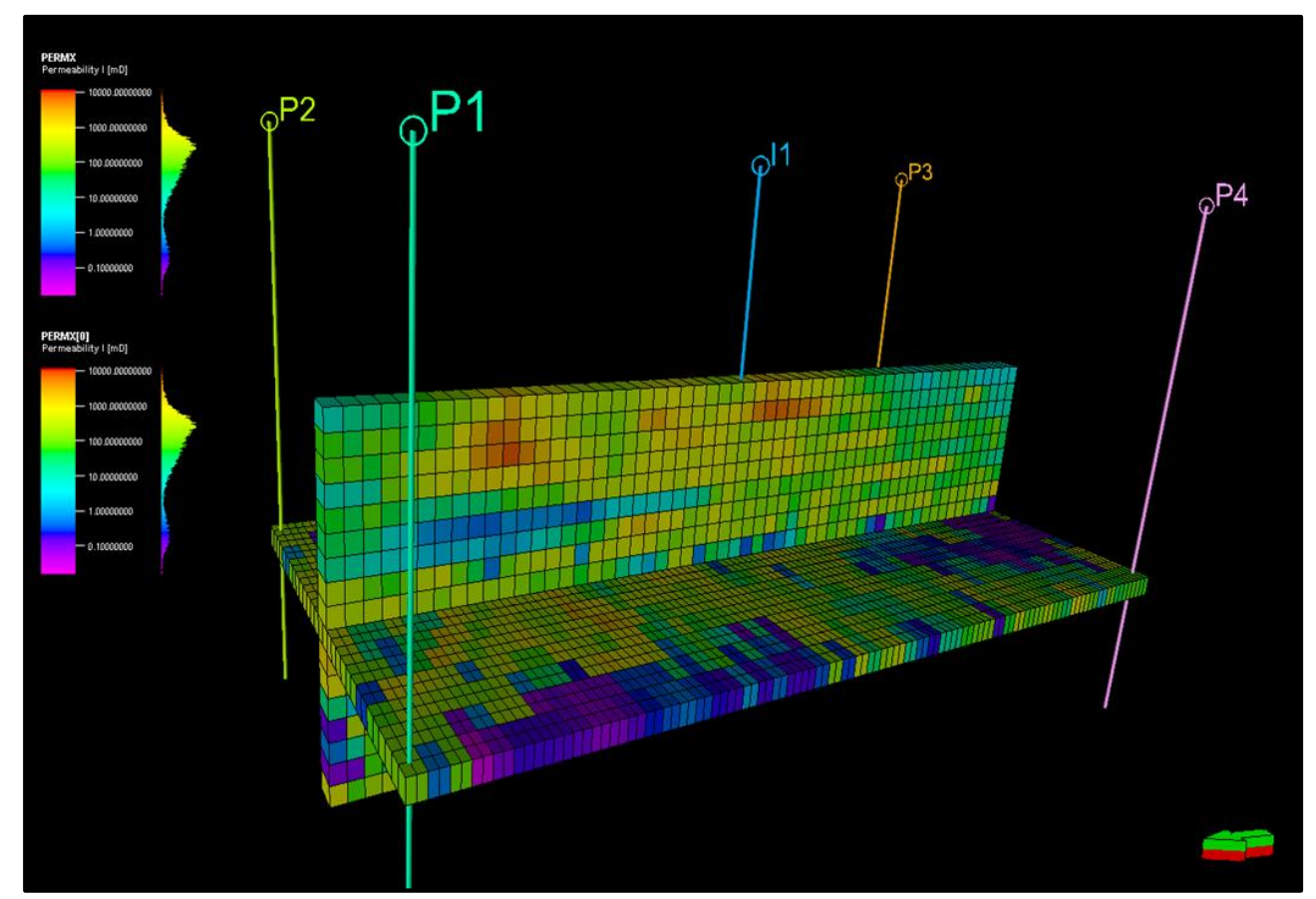

Figure 7-2: Permeability cross section of Fine Vs Cparse Grid.

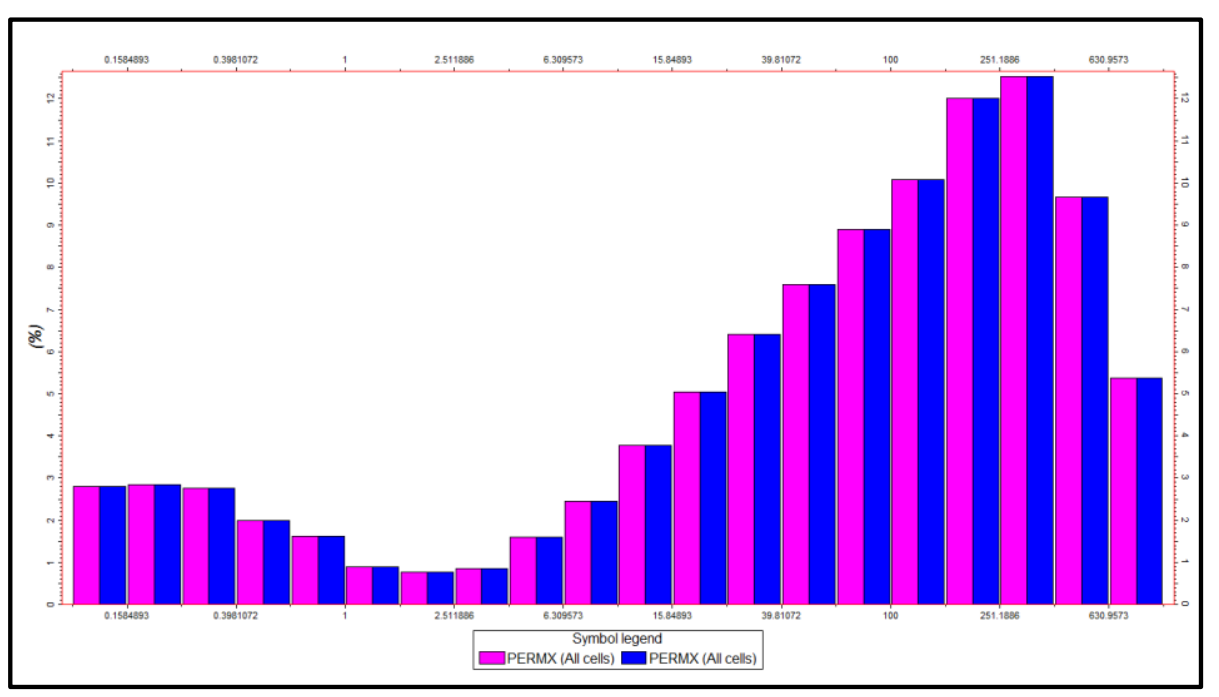

Figure 7-3: Permeability Histogram of the coarse Vs fine Grid. 
All other parameters were kept the same as the base case presented previously in chapter 6 . In this case, two simulation models were used that were the same as for the base case gas injection model, one for FD and another one for SL.

The new high-resolution gas injection model was tested and run for the full period. It is worth mentioning that on this case and other sensitivities, all the tuning parameters that were used in the base case gas injection will be used here without any modifications for the sake of consistent comparisons. A complete assessment and evaluation were also conducted to make sure that all the parameters from the coarse grid were still captured in the fine grid. Figure 7-4 presents the oil production rate forecasted in both the FD and SL cases. The figure illustrates the field oil production calculated by SL (red colour) and the same calculated by FD (black colour). As can be seen on the graph, the SL initial rate is slightly higher than FD initial oil production rate; this is mainly due to the pressure solver time steps used on SL, which usually takes larger time steps in comparison to FD calculations. Because of this, SL is much faster than FD.

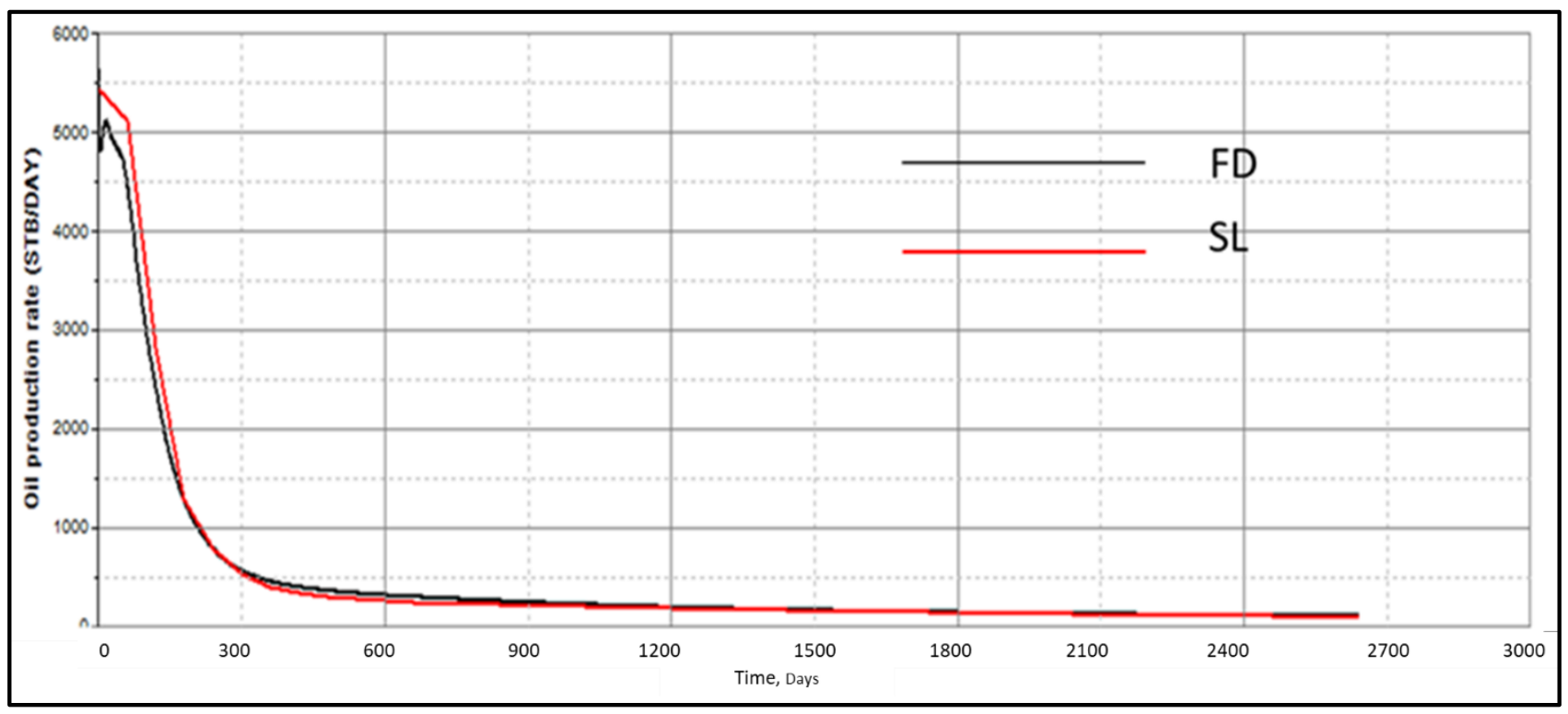

Figure 7-4: Oil production rate for both FD and SL (High Resolution Grid).

In the graph above, red colour presents oil production rate predicted by SL. However, black colour is the one calculated by FD. Initial oil rate calculated by SL is about 5300 bopd in compare to 5000 bopd calculated by FD. Means that 300 bopd difference noticed only in the initial rate. Then, that difference disappeared with time. 
Figure 7-5 presents the cumulative oil production forecasted for both cases, FD and SL. The difference in cumulative oil production comes actually as a result of the difference in oil production rates presented in the previous graph and as shown in graph below in the range of $5 \%$.

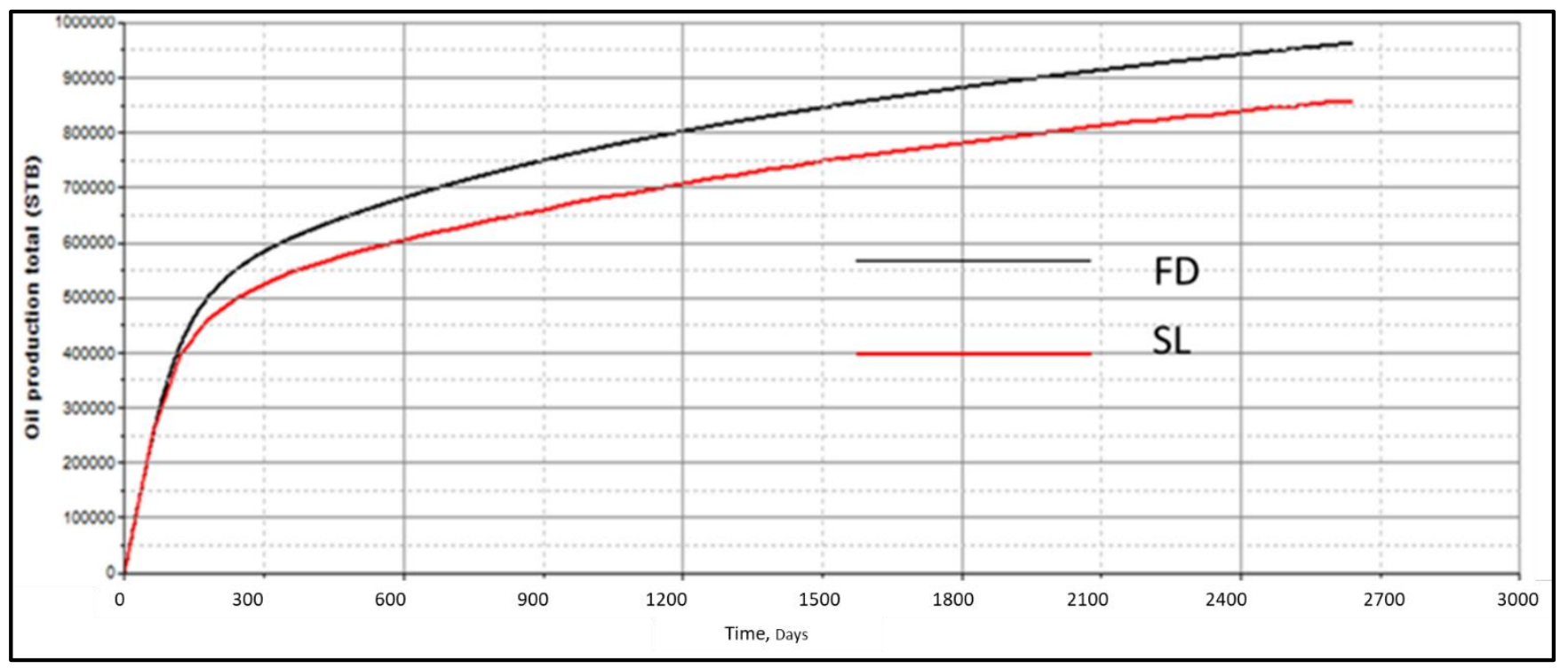

Figure 7-5: Cumulative Oil production rate for both FD and SL (High Resolution Grid).

Reservoir pressure performance is presented in Figure 7-6 for both cases, FD and SL. As can be seen from the plot, both trends are similar. However, the pressure in the SL simulation is slightly lower in comparison to the FD simulation. This difference is generally acceptable as the pressure field is usually solved in the conventional FD manner, and streamlines are traced from injector to producer. 


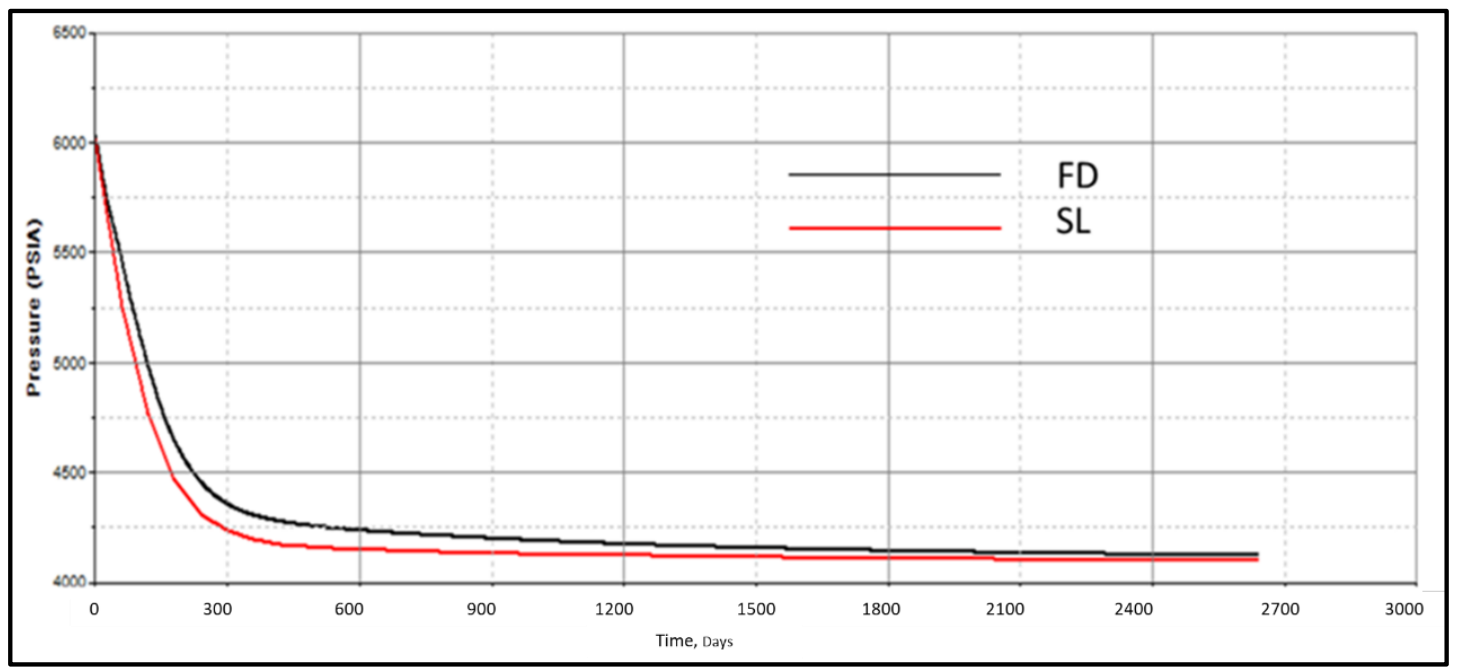

Figure 7-6: Pressure profiles for both FD and SL (High Resolution Grid).

Figure 7-7 presents gas injection rate performance of both FD and SL models. The figure shows that similar amounts of gas are used in the two simulation models.

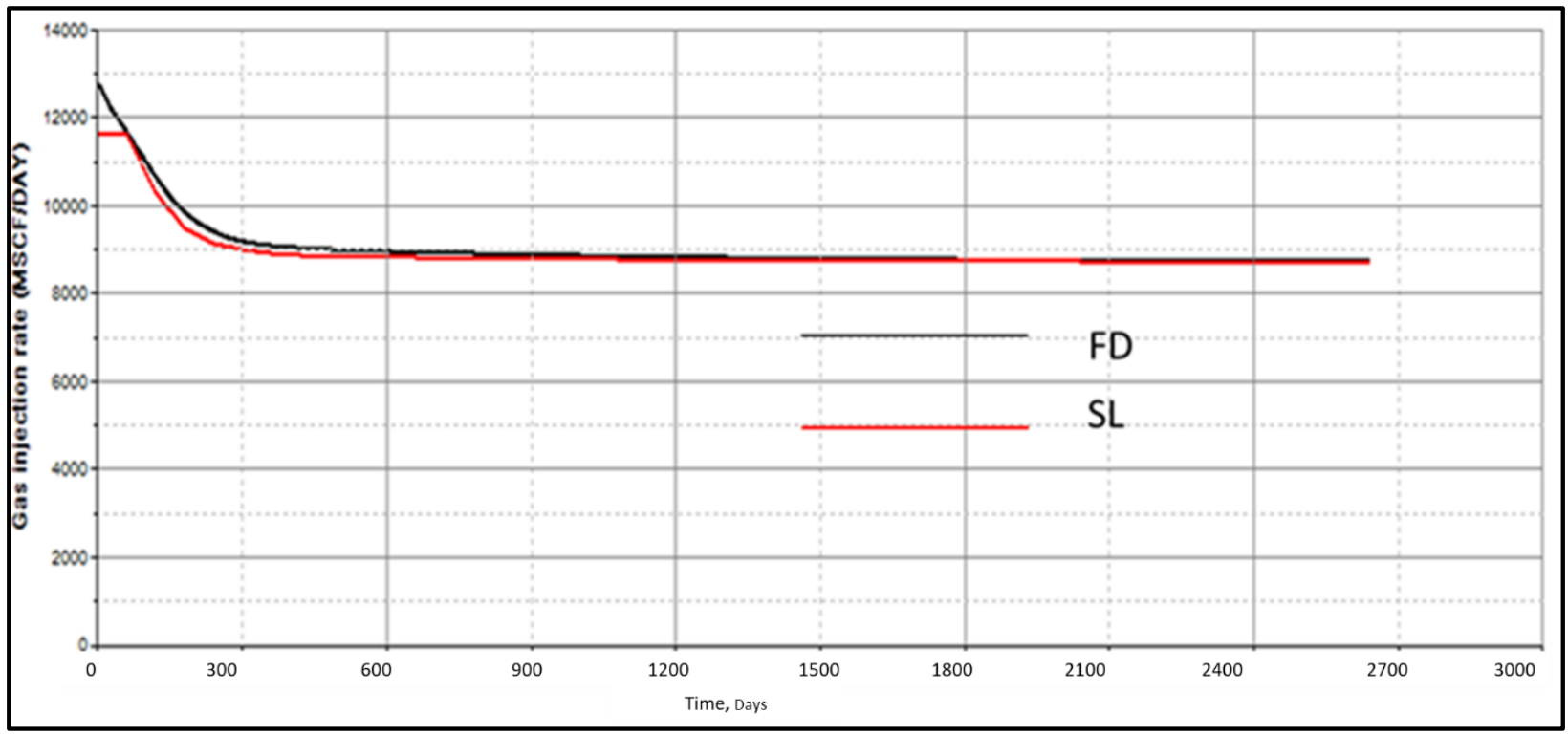

Figure 7-7: Gas Injection Rate Profiles for both FD and SL (High Resolution Grid).

Gas production rates are presented in Figure 7-8. The models show generally good agreement in the trend of gas production. However, there are some differences in the actual amount of gas produced, with FD giving slightly higher predictions in comparison to SL. 


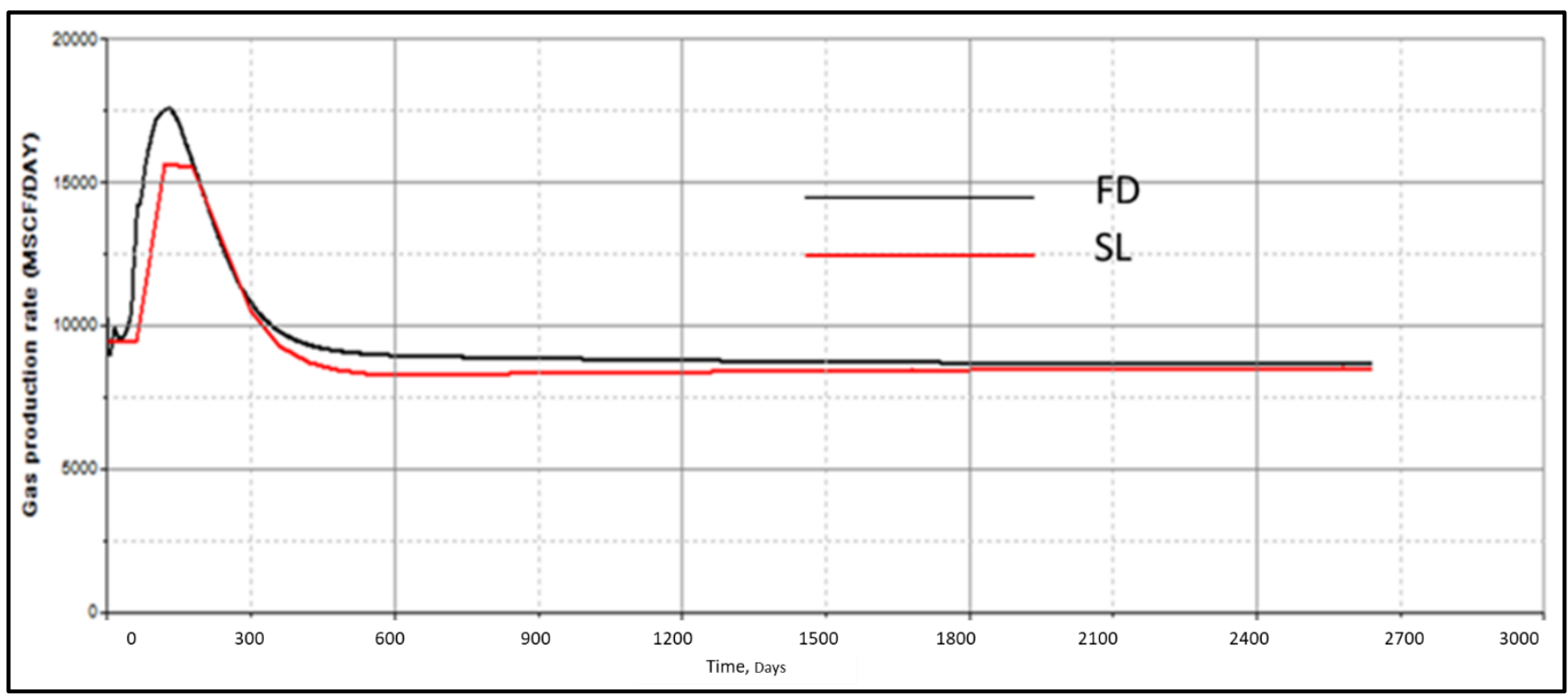

Figure 7-8: Gas Production Rate Profile for both FD and SL (High Resolution Grid).

The predicted water cut performance in both FD and SL simulation models is presented in Figure 79. In this case, the FD simulation predicts a smaller amount of water than SL. SL predicts a stable water production. However, FD initially predicts the same amount of water, and then steep decline is noticed until stabilisation is reached.

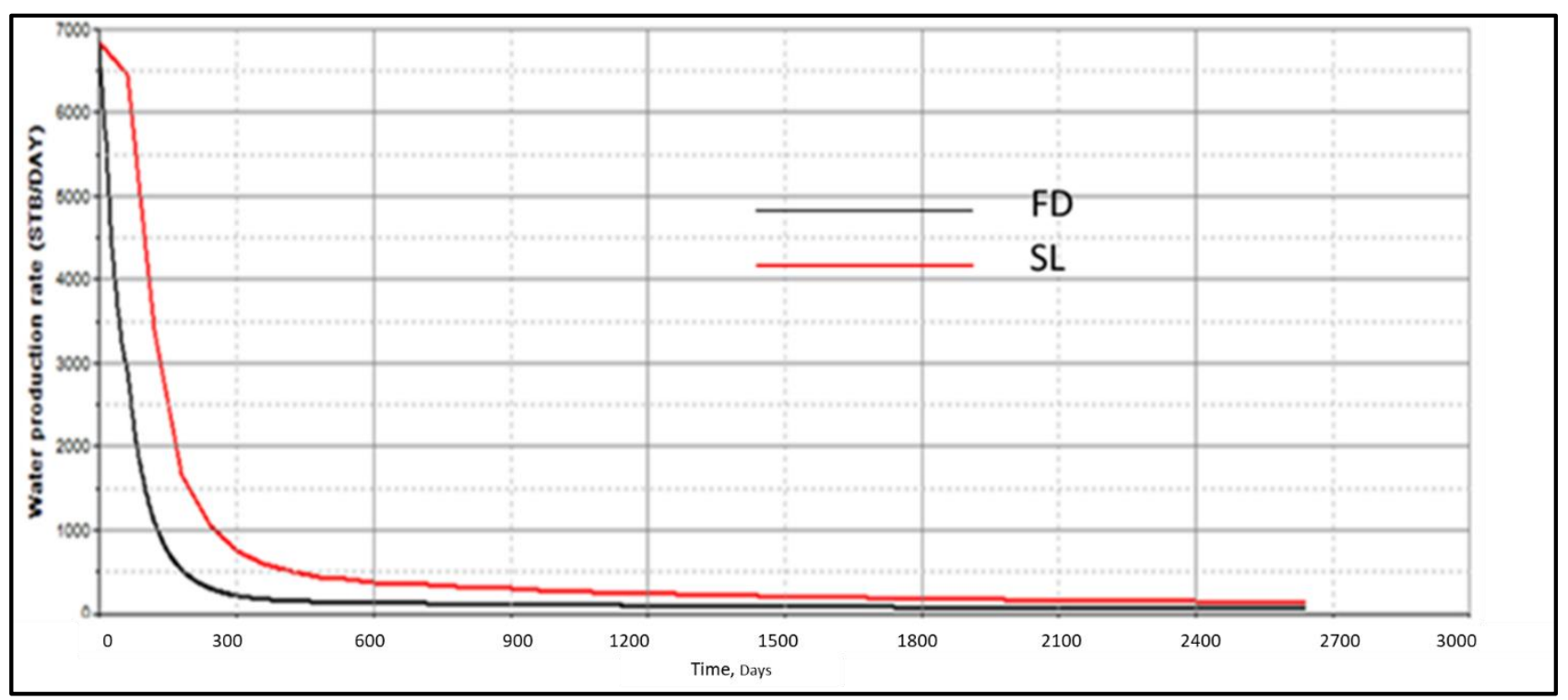


Figure 7-9: Water Production Profiles for both FD and SL (High Resolution Grid).

Comparing both models performances in terms of the computation time, Figure 7-10 presents the total CPU consumed by both FD and SL models. The FD simulation model consumed almost triple the amount of CPU in comparison to the SL simulation model.

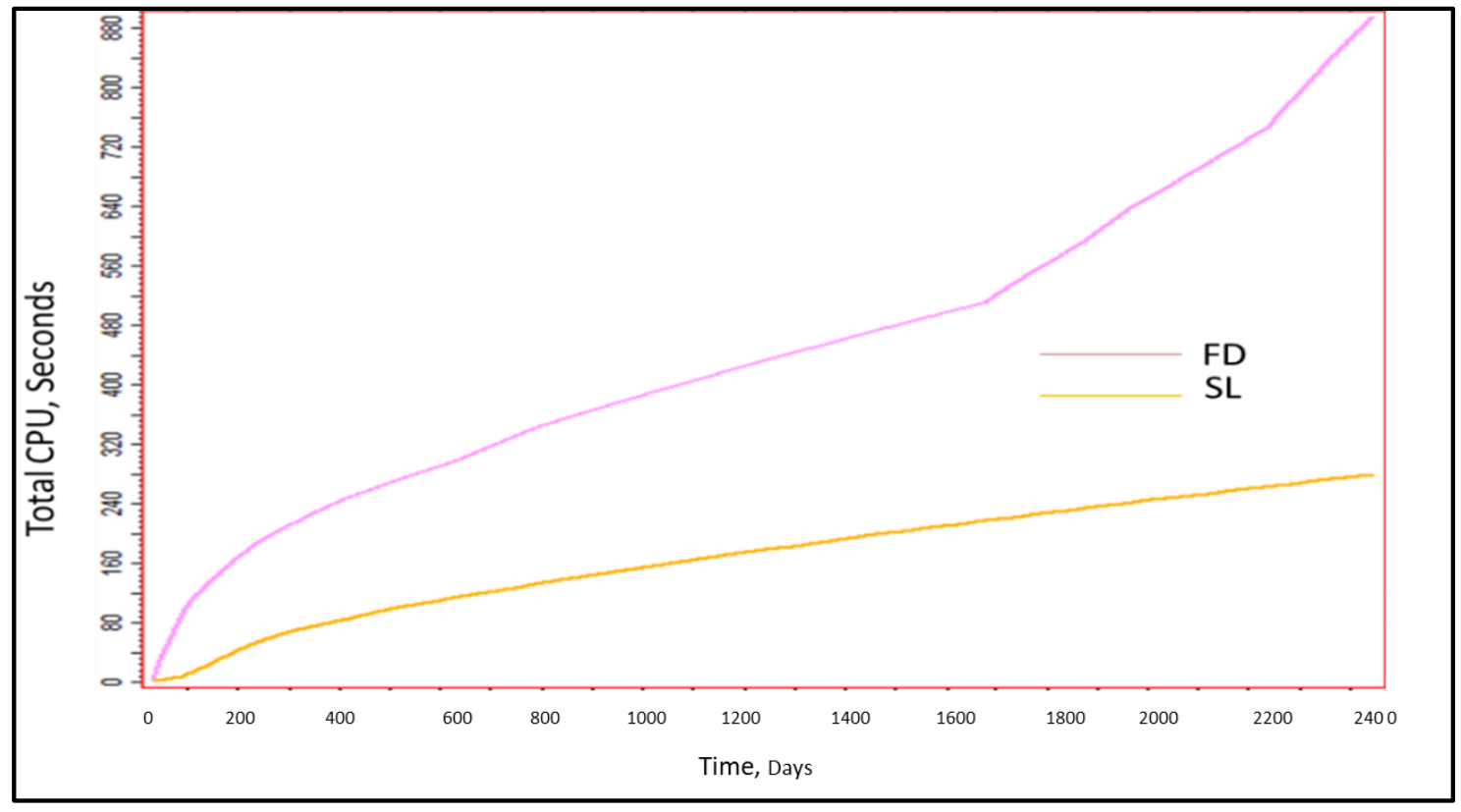

Figure 7-10: Total CPU required for FD and SL (High Resolution Grid).

All the results presented were for the high-resolution grid using the same tuning parameters as were used before on the previous chapter for the base line gas injection case. Detailed discussion and analysis for this case is presented in the following section 7.2.

The final result to be note here is that, for the high resolution grid model, the SL simulation works satisfactorily, and the results reveal a reasonable match with FD simulation, with the exception of differences in water production.

\subsection{Analysis of High-Resolution Grid}

To evaluate and assess the high-resolution grid sensitivity, the same tool presented in section 6.1 is used again here to analyse the high-resolution grid model. The results presented in this section are the results of the high saturation grid with the same tuning parameters used previously for the base case gas injection model. Figure 7-11 shows the relative error of oil rate for both FD and SL models. As can be seen from the plot, the error difference is $+/-7 \%$, occasionally increasing to $+/-15 \%$, but the average error is $7 \%$. In this case, we have used all the tuning parameters and constraints used previously in the coarse 
grid in chapter 6 and all model dimensions changed proportionally in order to keep similar oil in place. Hence, the deliverability of the wells is expected to be similar.

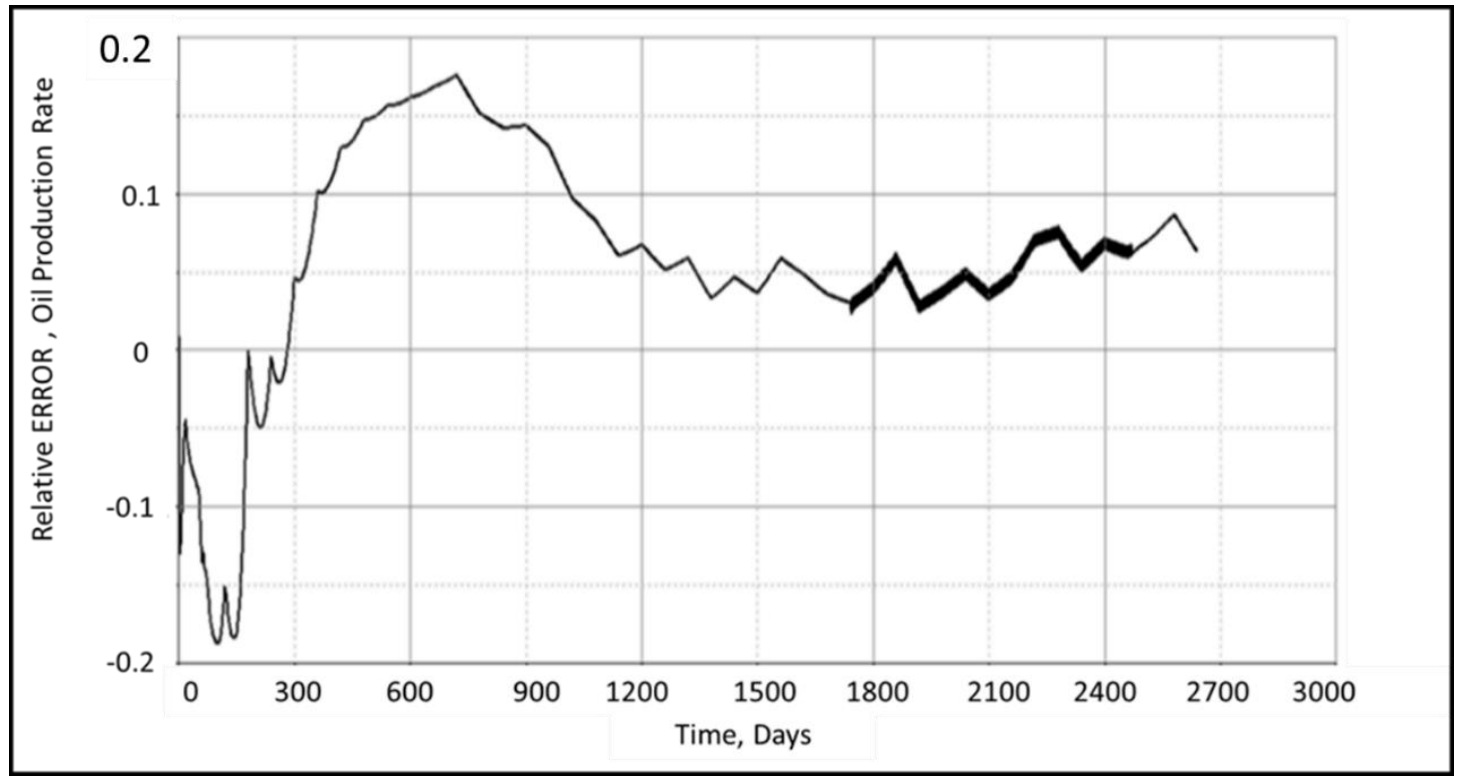

Figure 7-11: Relative Error in Oil Production Rate between FD and SL Simulation cases (High Resolution Grid).

Figure 7-12 shows the relative error of the total oil production; as can be seen from the plot the average relative error is quite high $(11 \%)$. 


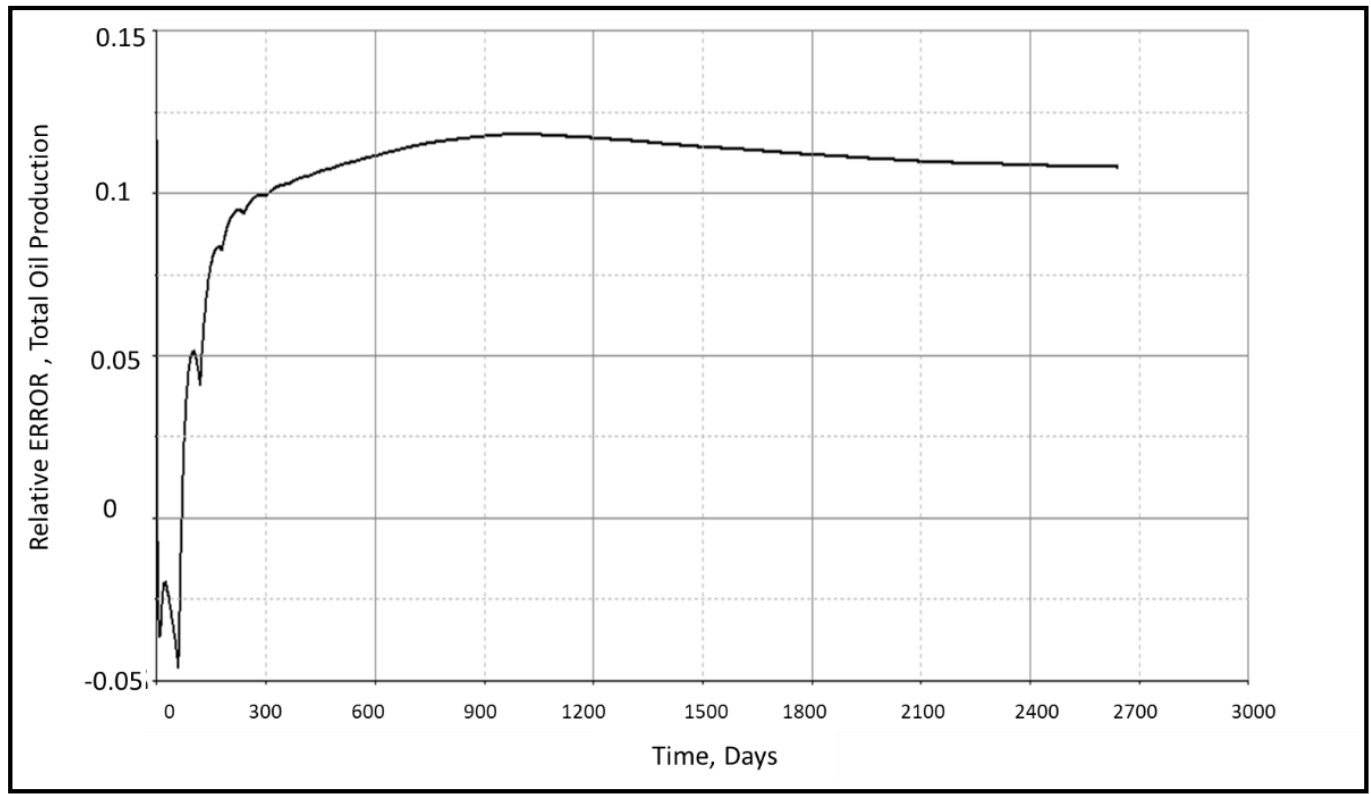

Figure 7-12: Relative Error of Total Oil Production between FD and SL Simulation cases (High Resolution Grid).

Figure 7-13 shows the relative error of the gas rate; as can be seen from the plot the average relative error is quite high (20\%).

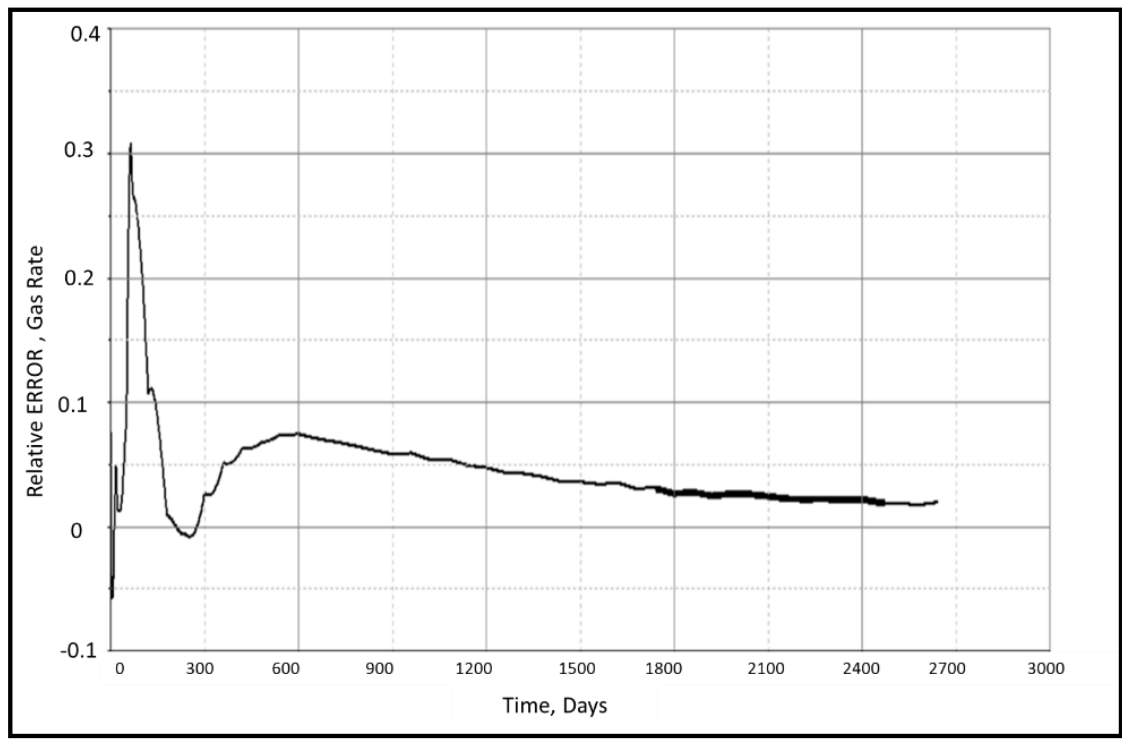

Figure 7-13: Relative Error of Gas Production Rate between FD and SL Simulation cases (High Resolution Grid). 
The relative error of the total gas injection is also calculated and presented in Figure 7-14. As can be seen from the graph, the relative error varies between $5 \%$ to $7 \%$, which affects both oil rate and total oil production. The average relative error in this case is $5 \%$.

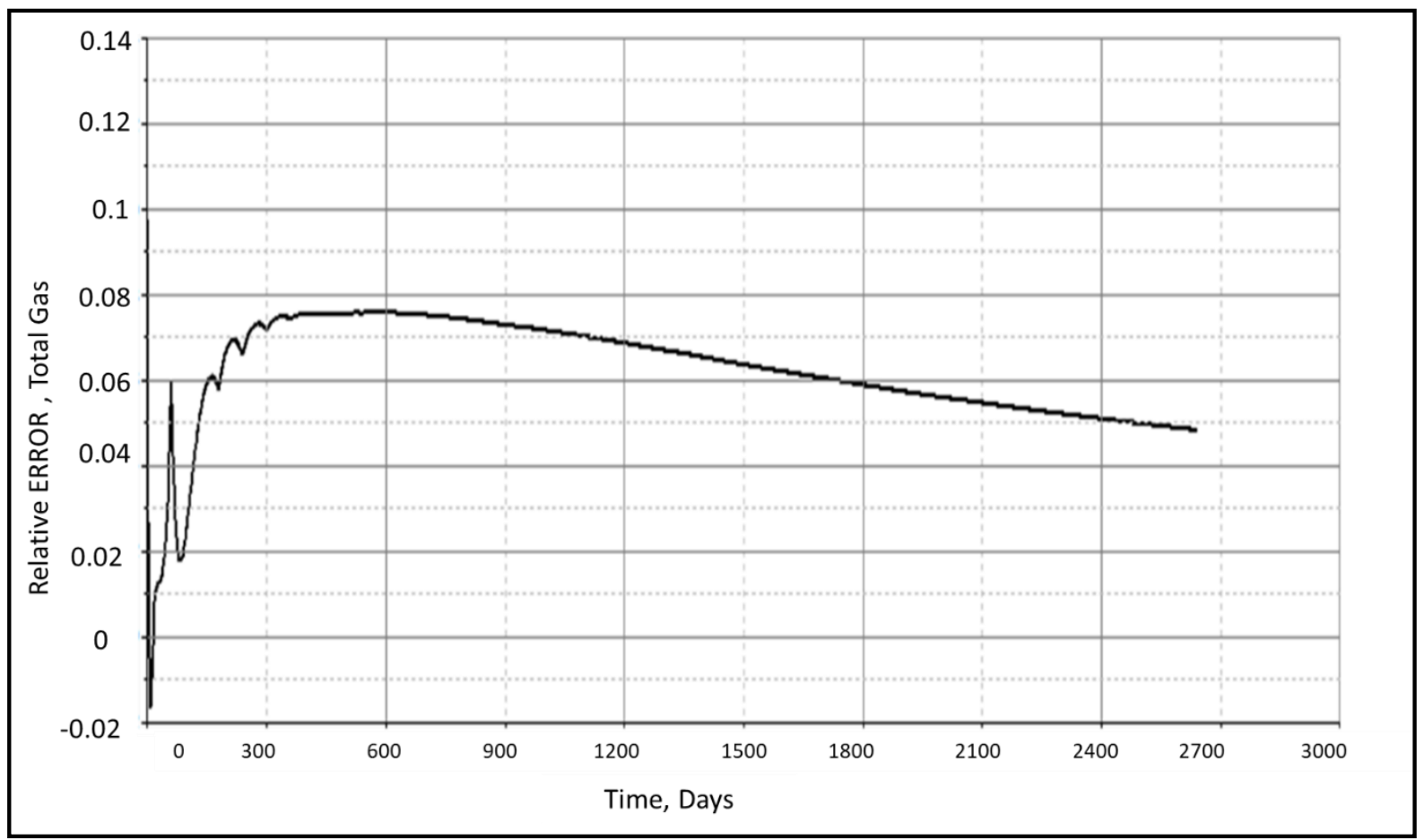

Figure 7-14: Relative Error of Gas Production Total between FD and SL Simulation cases (High Resolution Grid).

The relative error of the gas injection rate is calculated and presented in Figure 7-15. As can be seen from the graph, the relative error varies between $1.5 \%$ to $6 \%$, which affects both oil rate and total oil production. The average relative error in this case is $3 \%$. 


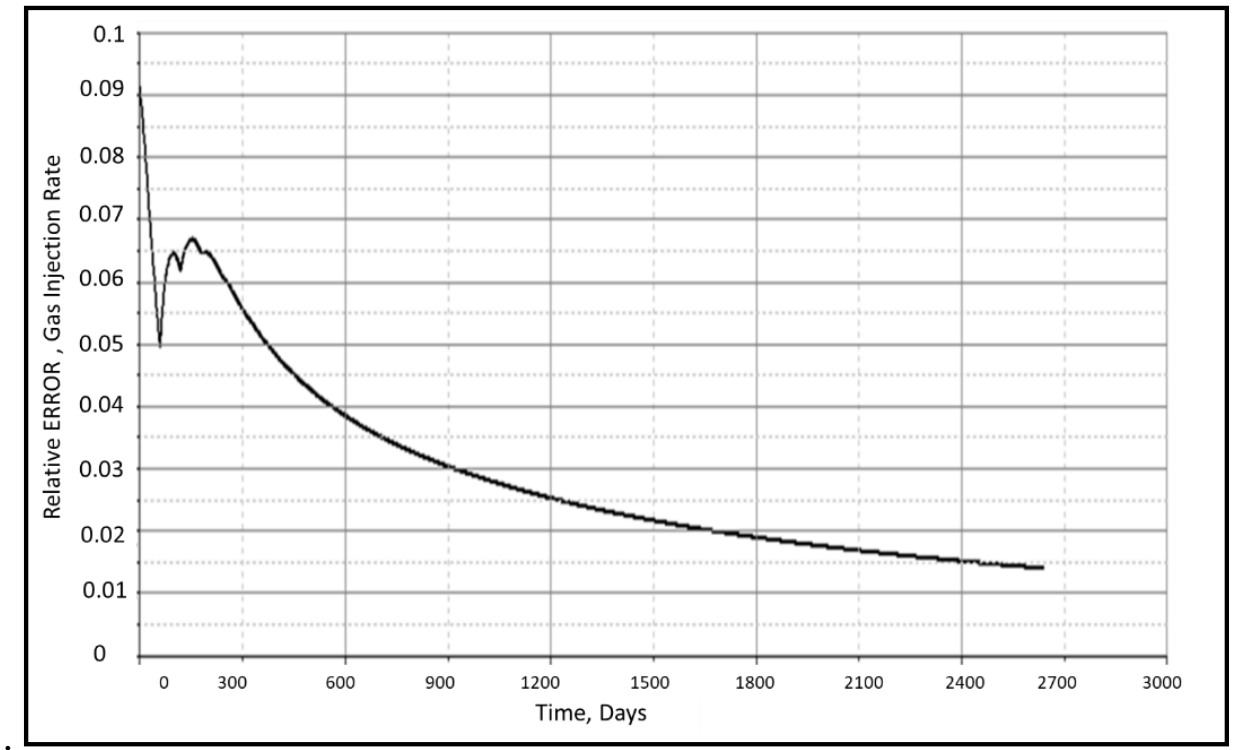

Figure 7-15: Relative Error of Total Gas Injection between FD and SL simulation models (High Resolution).

In terms of the relative error of the bottom hole pressure, it can be seen in Figure 7-16 that the average relative error of the pressure is $+/-2 \%$.

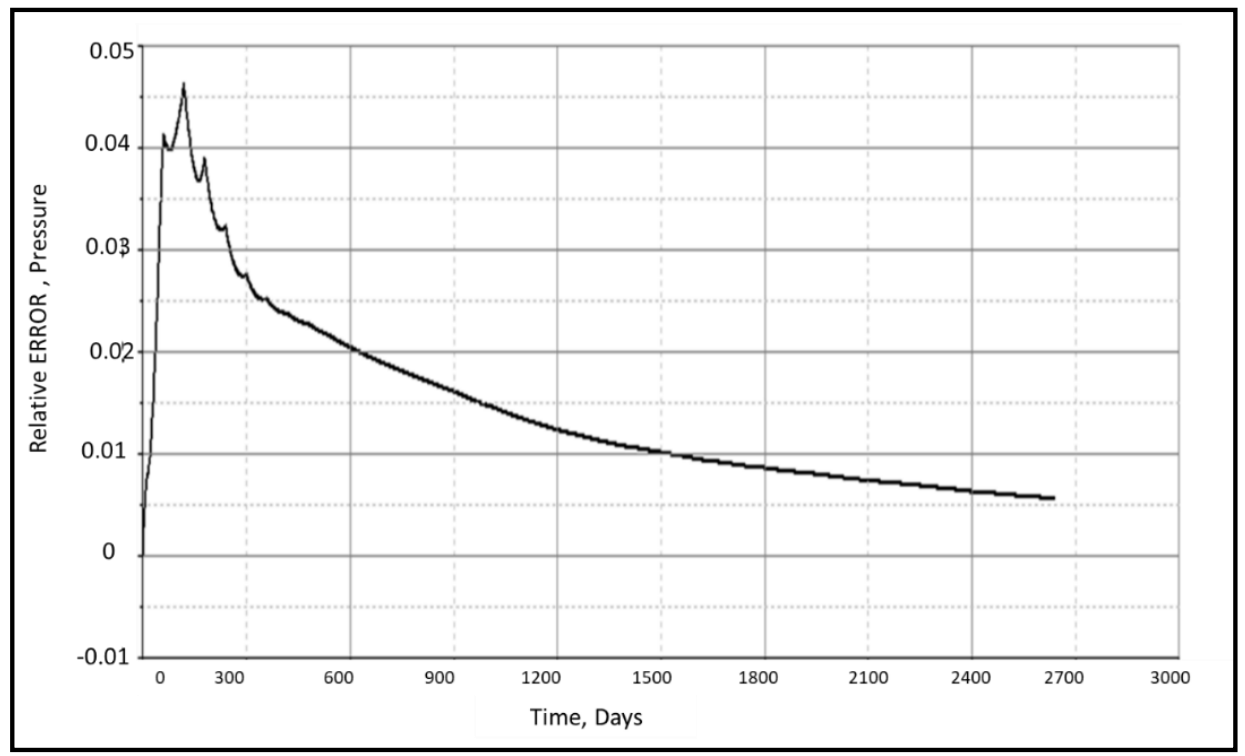

Figure 7-16: Relative Error of bottom hole pressure between FD and SL simulation models (High Resolution).

From all the analysis presented in the previous section, the relative errors appear to be high in comparison to the base case gas injection model. However, as it was mentioned earlier, no further tuning 
has been done on this case. The same tuning parameters used on the base case injection case are used here, in order to see the impact of these parameters. There is a room to improve this case and to minimise the relative error. However, in this exercise the conclusion is that using the same tuning parameters resulted in higher relative error. In order to improve SL performance model and hence reduce the relative error between SL and FD simulation models, well control models need to be refined again in both models to minimise the difference, especially in the initial production rates. If the initial production rates are similar, this will result in less relative error. Figure 7-17 presents the results before and after this improvement of the oil production rates.

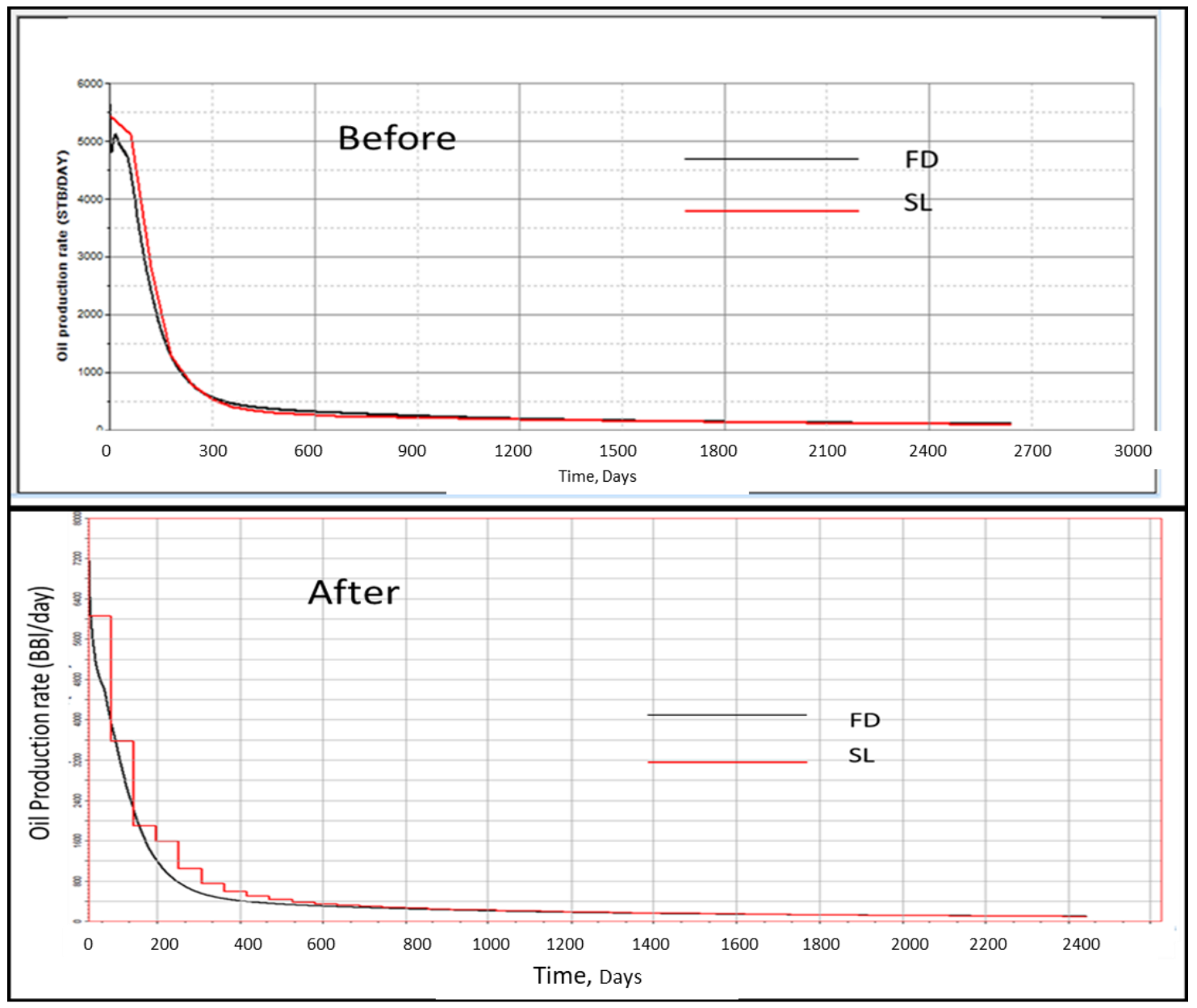

Figure 7-17: Oil Production Rates before and after improvement for both FD and SL models of the High-Resolution Grid. 


\subsubsection{D Analysis (High Resolution Case)}

With regards to the 3D grid analysis of high-resolution gas injection model (Black oil FD Vs SL Simulation), figure 7-18 below shows the 3D oil saturation grid at the first-time step on the left and on the right shows at the end of simulation for both FD black oil case in the top and SL model case in the bottom.

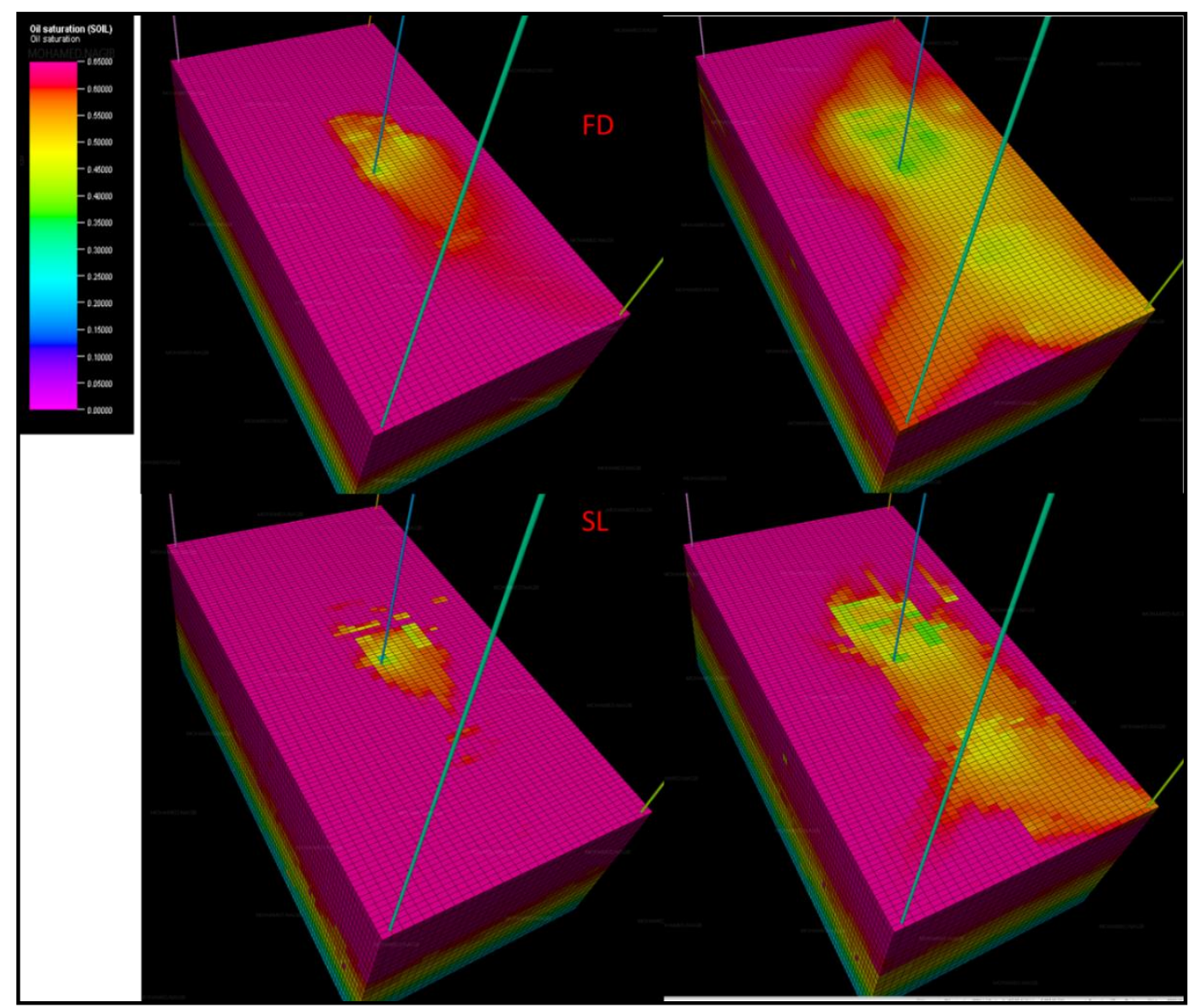

Figure 7-18: First- and Last-time steps 3D Oil saturation Grid (High Resolution FD Model)

Similar trends in both models were observed as can be seen from the graph above. Sliced 3D oil saturation grids are presented in figures 7-19 below for both FD and SL high resolution gas injection model. Similar conclusion obtained as well from this comparison as concluded earlier in 3D grids above. 


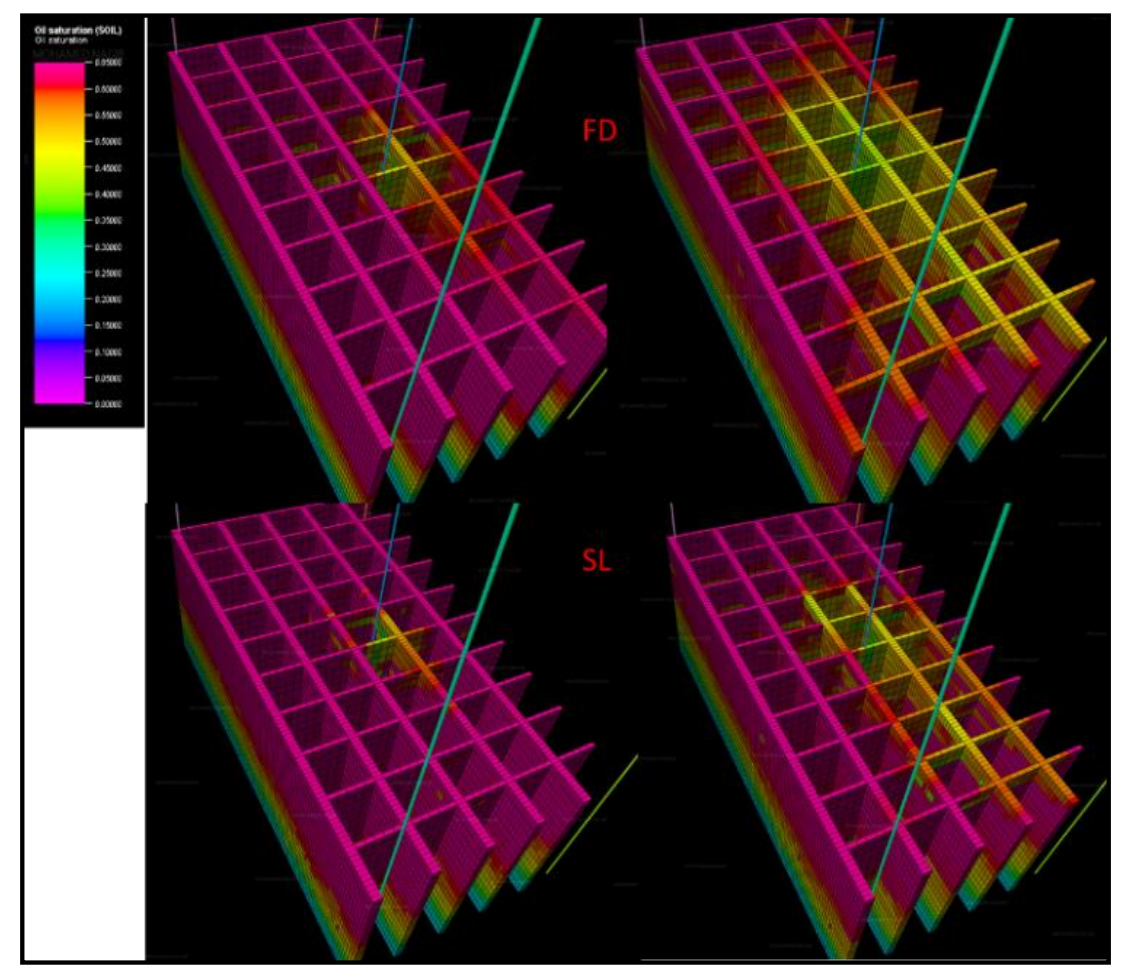

Figure 7-19: First- and Last-time steps 3D Oil saturation Grid (High Resolution SL Model)

Cross section 3D oil saturation grids are presented in figures 7-20 for both FD and SL high resolution gas injection models. Similar conclusion obtained as well from this comparison as concluded earlier in $3 \mathrm{D}$ grids above. 


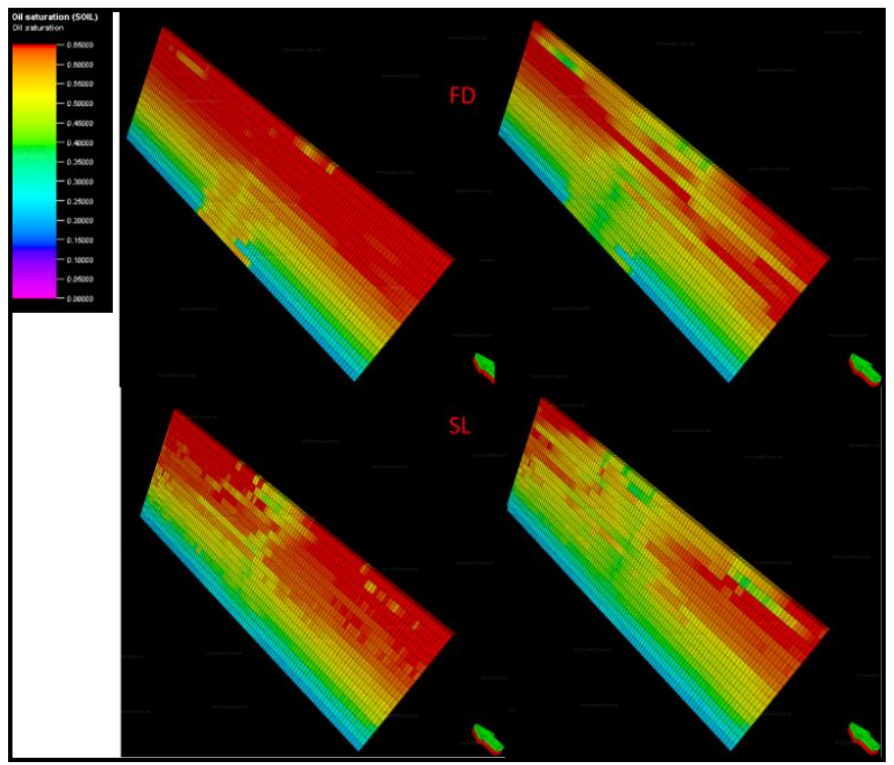

Figure 7-20: First- and Last-time steps of cross section 3D Oil saturation Grid (High Resolution Model)

Similar analysis done as well for gas saturation. Base case FD and SL 3D gas Saturations at firstand last-time steps is presented in figure 7-21. Similar trends can be noticed from 3D gas saturation Grids below. 


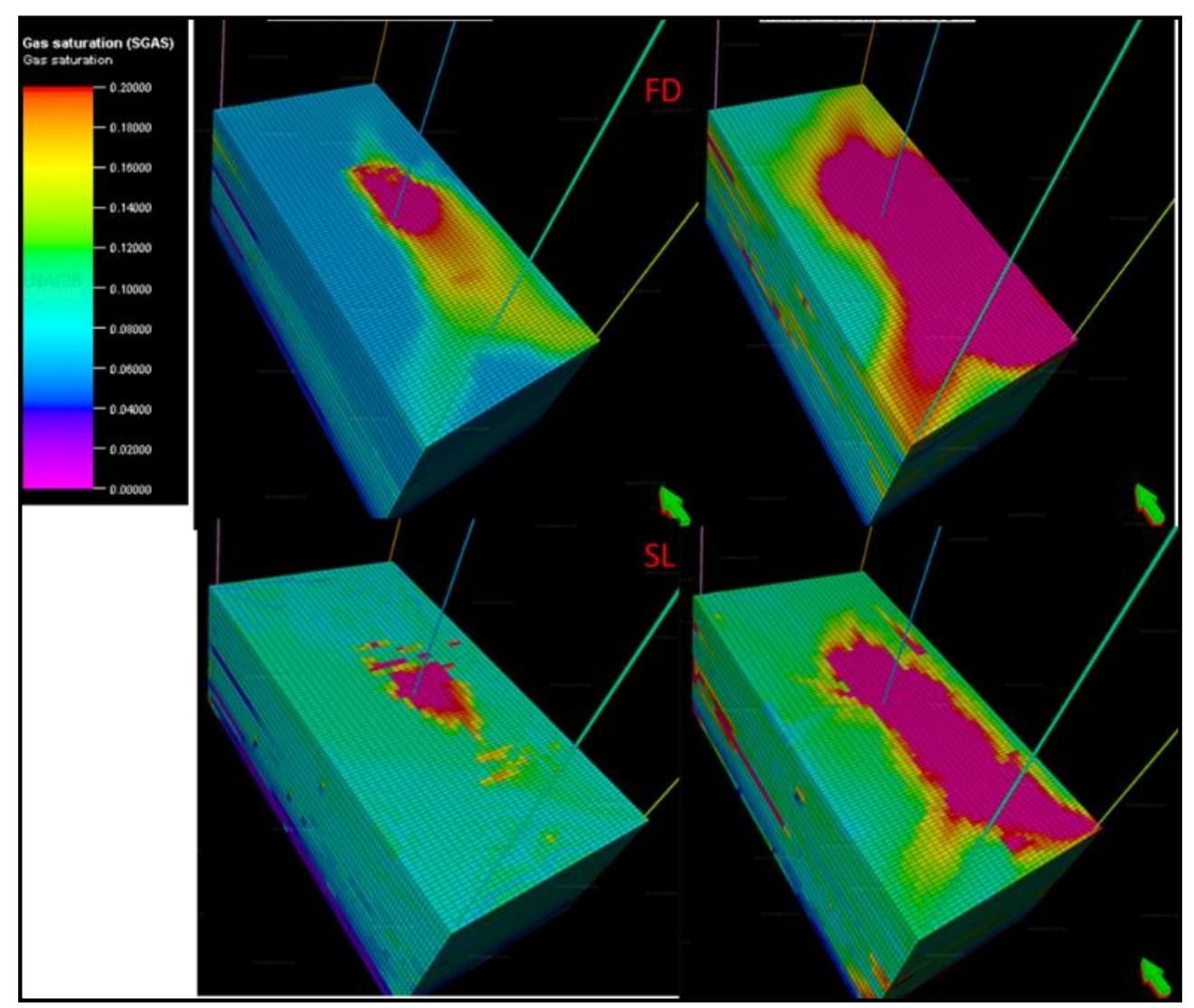

Figure 7-21: First- and Last-time steps of 3D Gas Saturation Grid (High Resolution Model)

Sliced 3D gas saturation grids are presented in figures 7-22 for both FD and SL high resolution gas injection models. Similar conclusion obtained as well from this comparison as concluded earlier in 3D grids above. 


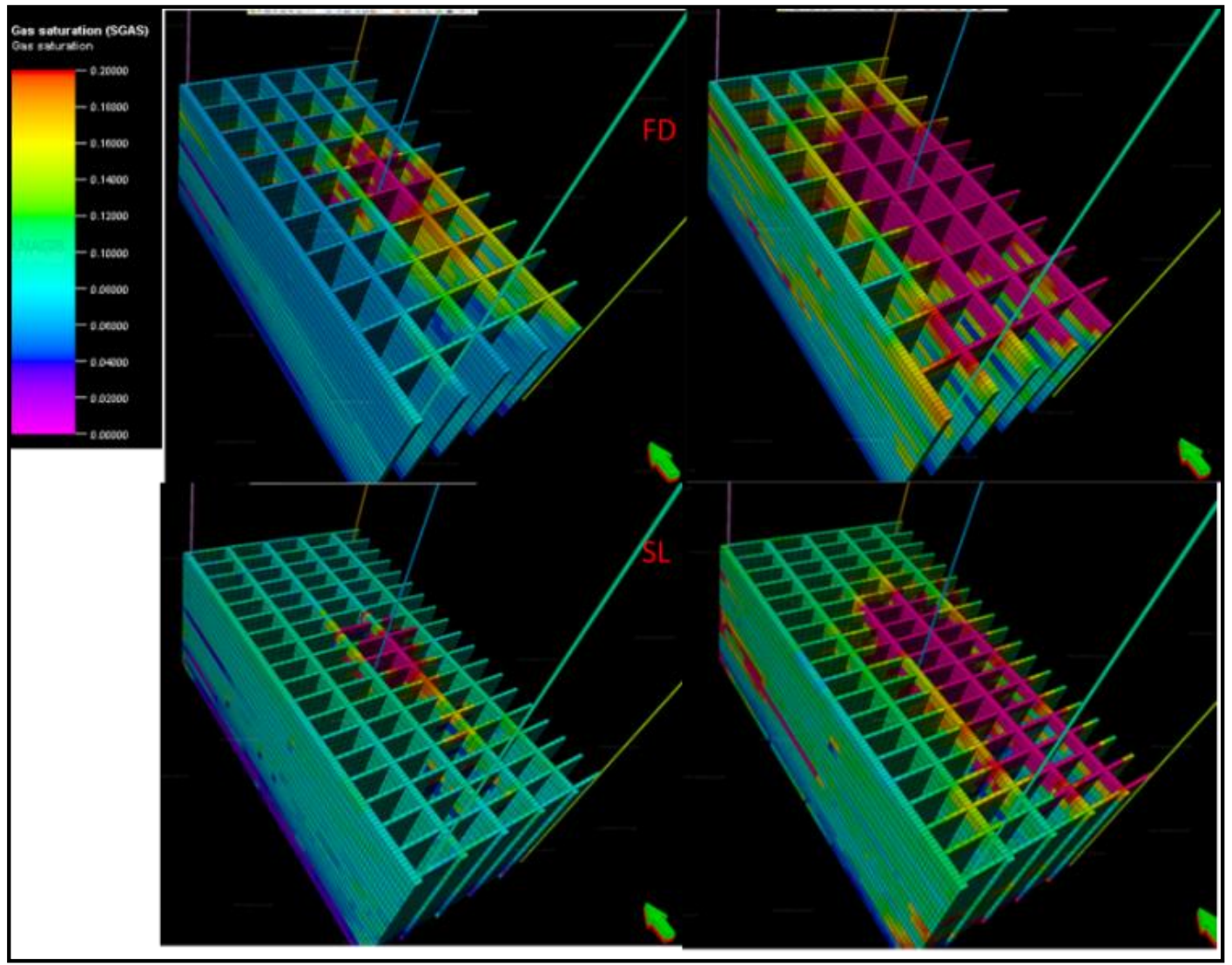

Figure 7-22: First- and Last-time steps of sliced 3D Gas Saturation Grid (High Resolution Model)

Cross section 3D gas saturation grids are presented in figures 7-23 for both FD and SL High resolution gas injection model. Similar conclusion obtained as well from this comparison as concluded earlier in $3 \mathrm{D}$ grids above. 


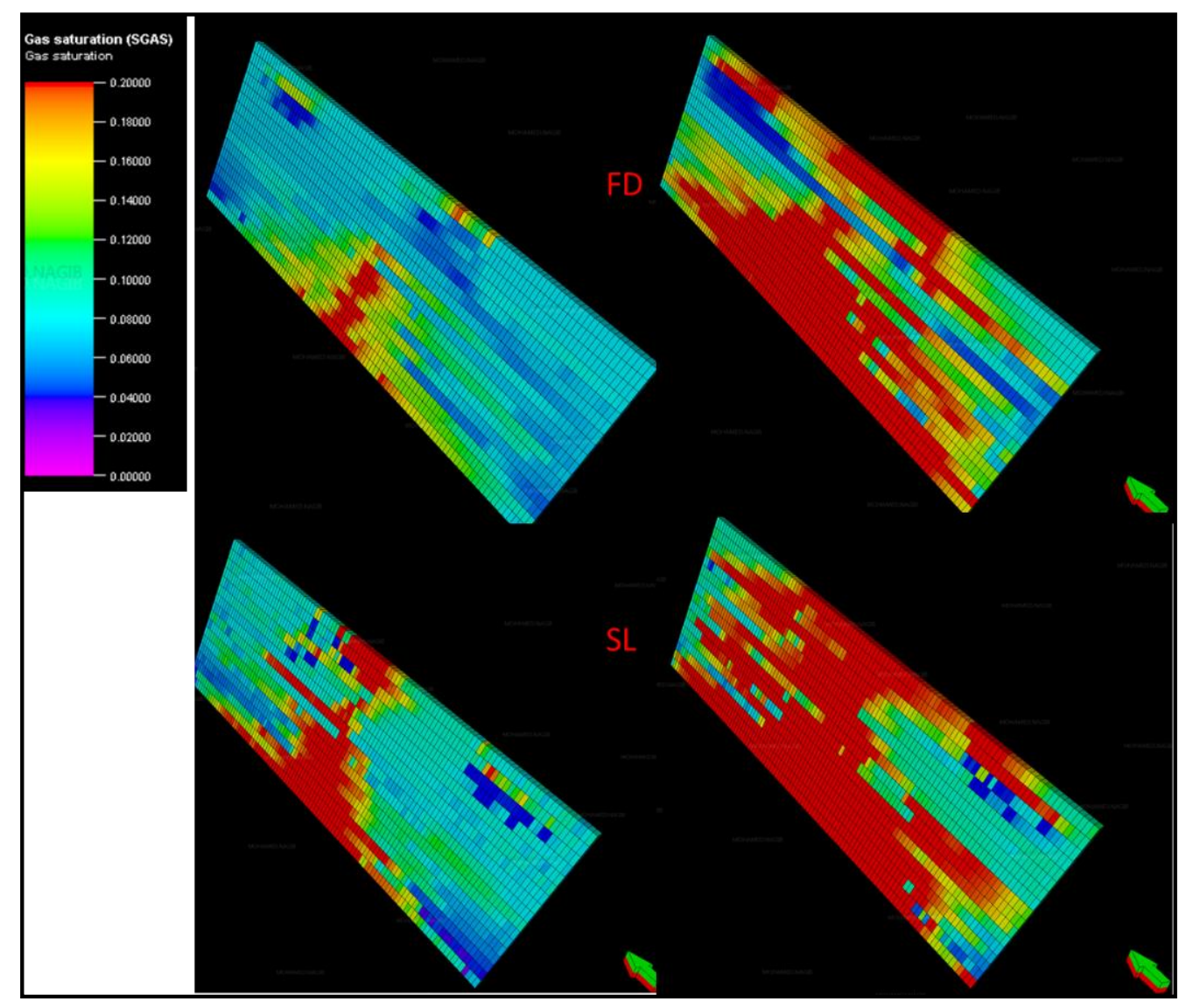

Figure 7-23: First- and Last-time steps of Cross section 3D Gas Saturation Grid (High Resolution Model)

Similar trends are noticed in gas saturation changes in both FD and SL models for high resolution case as shown on the above figures.

As mentioned earlier in chapter 6, Oil Thickness Column (OTC) at the end of the field life and this property is calculated for both FD and SL grids according to its definition in Equation (6.2). Figure 7-24 shows OTC at the end of field life of both FD and SL models. 


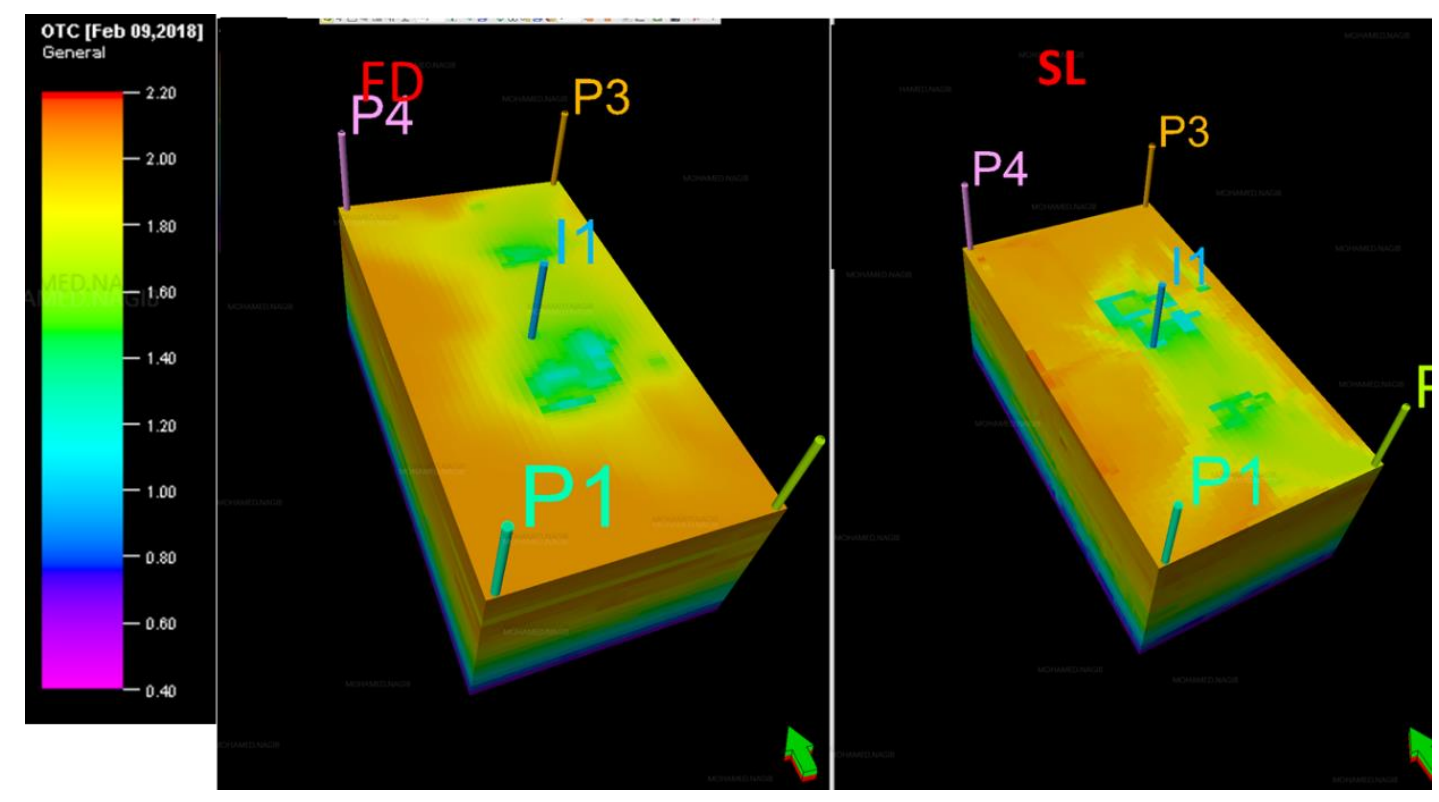

Figure 7-24: High Resolution Grid OTC of both FD and SL Models

\subsection{Conclusions}

- A high-resolution grid of the same base case gas injection model was developed by creating a new grid with the properties and increasing the number of grid cells in the $\mathrm{x}$ and $y$ directions by twice the number of the base case.

- The tuning parameters were kept the same in this case in order to see the quality and the degree of match between the base case and high-resolution grid.

- Oil and water production rates comparisons showed similar performances were observed in both FD and SL high resolution gas injection models.

- Oil saturation grids comparisons are showing similar trends with minor differences in both FD and SL high resolution models.

- In terms of computational times comparisons, FD simulation model consumed almost triple the amount of CPU in comparison to the SL simulation model.

- Both FD and SL models showed some sort of instability, which resulted in higher relative errors in comparison to the base case. 


\section{Chapter 8}

\section{Compositional and Miscible Flood Models}

In this section, the base case gas injection model will be modified to the compositional model and miscible flooding cases to test and assess SL modelling and compare the results with FD modelling.

\subsection{Compositional modelling}

The base case gas injection model was modified to run in compositional mode in both conventional FD and SL simulation. PVT and relative permeability data in the base case were replaced by compositional data in order to test and evaluate SL accuracy in predicting production performance, and to compare the results with conventional compositional FD simulation.

For compositional modelling, the Equation of State (EOS), is required to define gas compositions and Ncomp is the key word required to specify the number of components and to support the three-phase compositional mode. Several EOS are available to use in the simulation. Either Peng Robinson, RedlichKwong, Soave-Redlcich Kwong or Zudkevitch-Joffe - Redlich-Kwong could be used.

Compositional modelling is usually implemented in $3 \mathrm{~d}$ two phase streamline bases with the following procedure or protocol:

1. Initialization.

2. Pressure solution on the grid considering compressibility.

3. Calculation of porosities and saturations for the new pressure fields, keeping constant mass concentrations.

4. Mass transport solution by means of streamlines. Streamlines traced from injector to producers.

5. New grid saturation calculations according to compressibility and new mass concentrations.

6. Mass concentrations solution for gravity.

7. Calculation for new grid saturations.

A flow chart for compositional streamline simulator is presented Figure 8-1 [33]. 


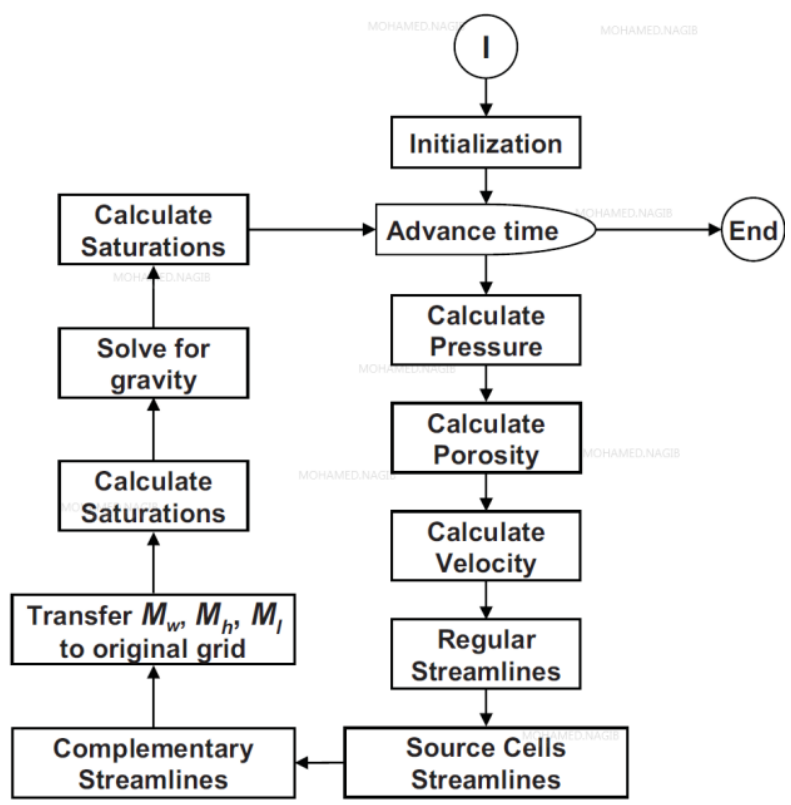

Figure 8-1: Compositional streamline simulator flow chart.

The thermodynamic equilibrium between phases must be considered. In addition to that, the initial saturations must be in equilibrium. In Eclipse 300 (FD simulator), the ZMFVD option is used to specify the initial composition with respect to depth and gas-oil contact may be set above or below the reservoir if the single-phase hydrocarbon is an oil or gas, simulator sets the phase identification method to produce a phase change at this depth. For cases in which there is a gas-oil contact within the reservoir, and the vapor composition is known. ZMFVD also used to specify the initial vapor composition. In this work, Peng Robinson is used as EOS and the number of components is 6 as per table 8-1.

Table 8-1: Compositional PVT data set.

\begin{tabular}{|c|c|c|c|c|c|}
\hline Components & $\begin{array}{c}\text { Critical } \\
\text { Temperature, } \\
\text { Deg K }\end{array}$ & $\begin{array}{c}\text { Critical } \\
\text { Pressure, } \\
\text { Psi }\end{array}$ & $\begin{array}{c}\text { Critical } \\
\mathbf{Z} \\
\text { Factor }\end{array}$ & $\begin{array}{c}\text { Molecular } \\
\text { Weight }\end{array}$ & $\begin{array}{c}\text { Acentric } \\
\text { factor }\end{array}$ \\
\hline C1 & 343 & 668 & 0.29 & 16 & 0.01 \\
\hline C3 & 666 & 616 & 0.28 & 44 & 0.15 \\
\hline C6 & 913 & 437 & 0.26 & 86 & 0.3 \\
\hline C10 & 1112 & 304 & 0.26 & 149 & 0.49 \\
\hline C15 & 1270 & 200 & 0.25 & 206 & 0.65 \\
\hline C20 & 1380 & 162 & 0.24 & 282 & 0.85 \\
\hline
\end{tabular}


Figure 8-2 below represents the relative permeability data used for compositional modelling.

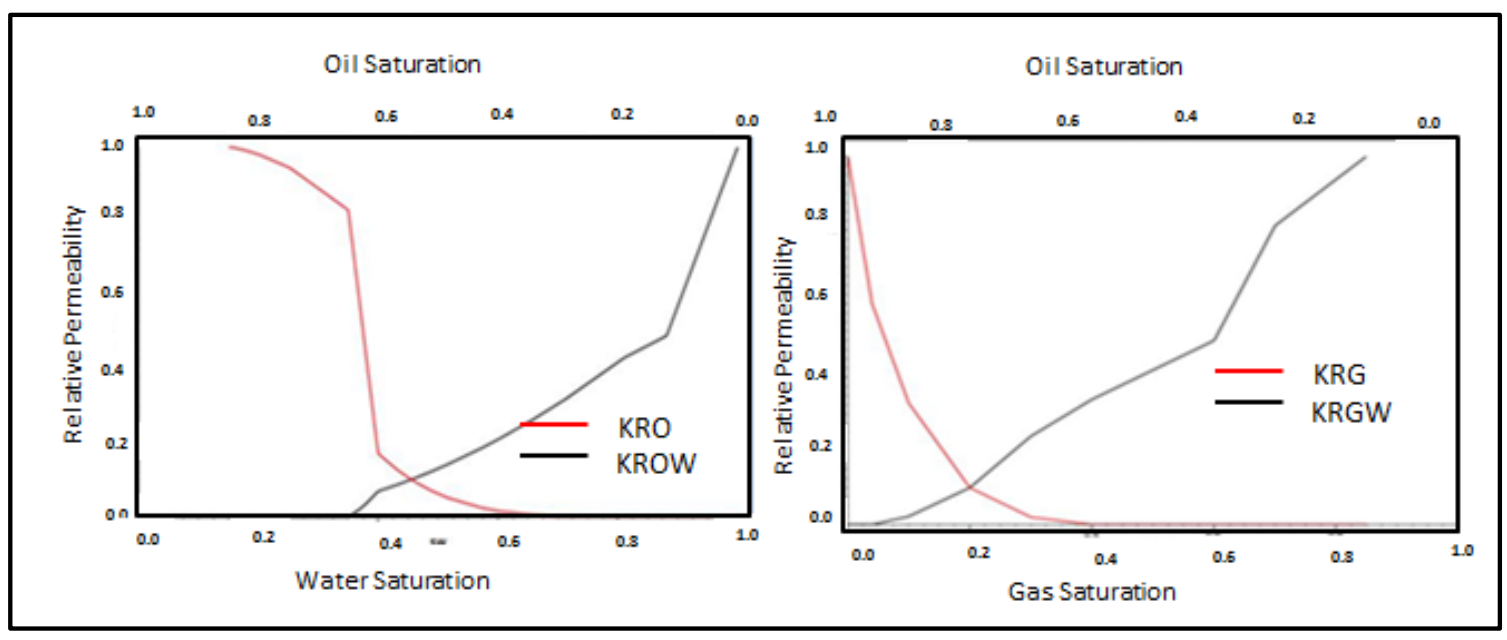

Figure 8-2: Compositional modelling relative permeability data used in both FD and SL simulations.

\subsubsection{Tunings required for compositional Streamline simulation}

In compositional streamline, the saturation solution is tuned with keyword TUNEFSSA as the frequency of Segregation Iterations within each timestep is controlled with item 1. The most important parameter affecting the speed of computations is the Streamline Density multiplier after timestep length. It is impacting the amount of memory required to solve a problem. This can be switched on automatic streamline density computation by setting this value to -1 .

For the 1D solver, Key word TUNEFS1D is used to set the tuning options for three-phase, this only affects three-phase runs. When set to 1, FrontSim (SL Simulator) will allow more than one iteration on gravity segregation using the first parameter. This tuning key word are used also determine the number of streamlines required Nstreamlines, and maximum number of streamlines calculated simultaneously in a single call to $1 \mathrm{D}$ solver.

The results of the compositional modelling streamline simulation are presented in the following section. For the oil production rate, Figure 8-3 presents the oil production rate forecasted in both cases of FD and SL. The figures illustrate the field oil production calculated by SL (red colour) and same calculated by FD (black colour). As can be seen in the plots, there is an overall good match in the initial oil production rate 
calculated using FD simulation in comparison to SL. The FD performance is stable; however, the SL performance shows some small instabilities. This could be due to the saturation solver when mapping the variation of the change while displacing the oil inside the model in a full compositional mode.

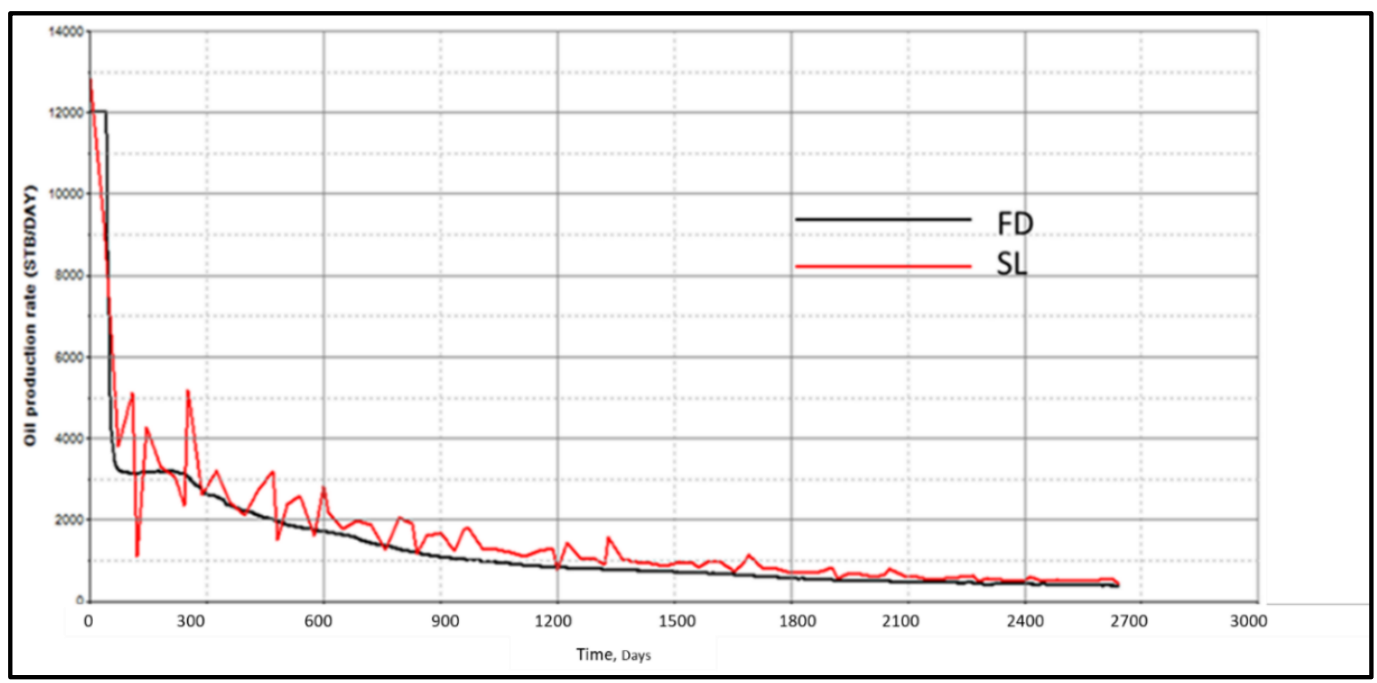

Figure 8-3: Oil production rate for both FD and SL (Compositional).

For cumulative oil production, Figure 8-4 presents cumulative oil production forecasted in both FD and SL cases. In this graph, cumulative oil production is calculated by SL (red colour) and the other one is calculated by FD (black colour). As can be seen, a slight mismatch at the end of the production period is observed, and this is mainly due to the stability of the initial oil production rate calculated using FD, which results in a slight increase in total production at the end of the field life.

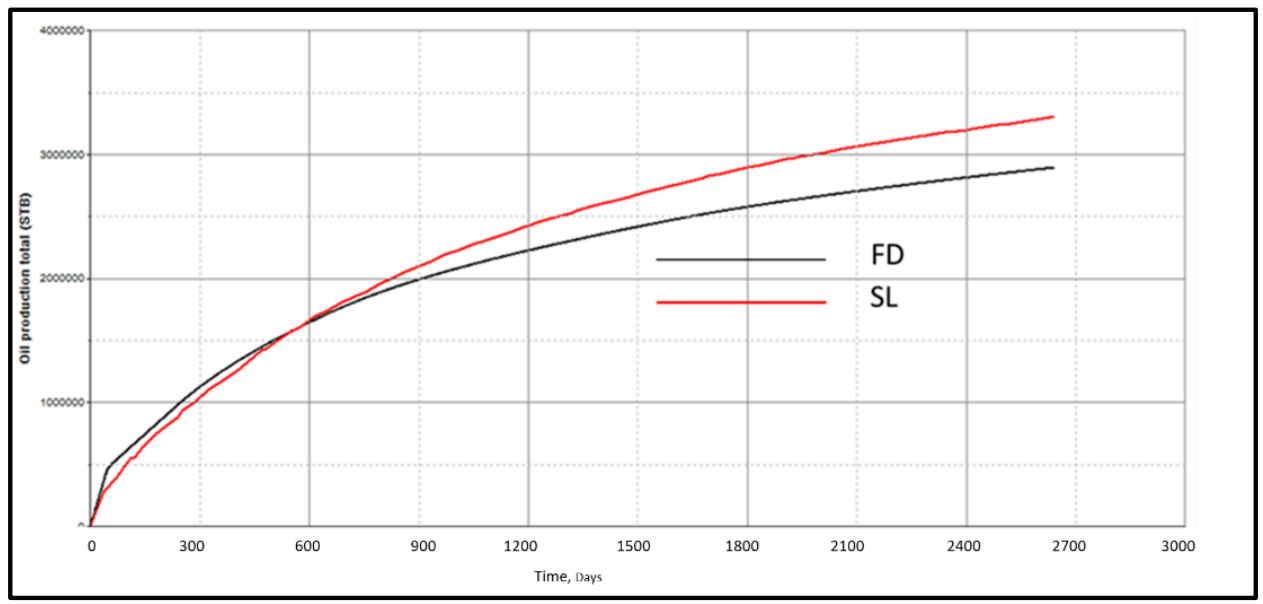

Figure 8-4: Cumulative Oil production for both FD and SL (Compositional) 
Figure 8-5 below represents gas production rates calculated in both FD and SL models. As can be seen from the plot, the FD model displays smooth evolution, whereas the SL model displays large oscillatory instabilities. However, the average values of the SL model follow the same trend as the FD model, though the values lie lower, becoming closer for time steps greater than 2100. Constant gas production rate was noticed in the early period of the FD model same as noticed earlier for the oil production rates.

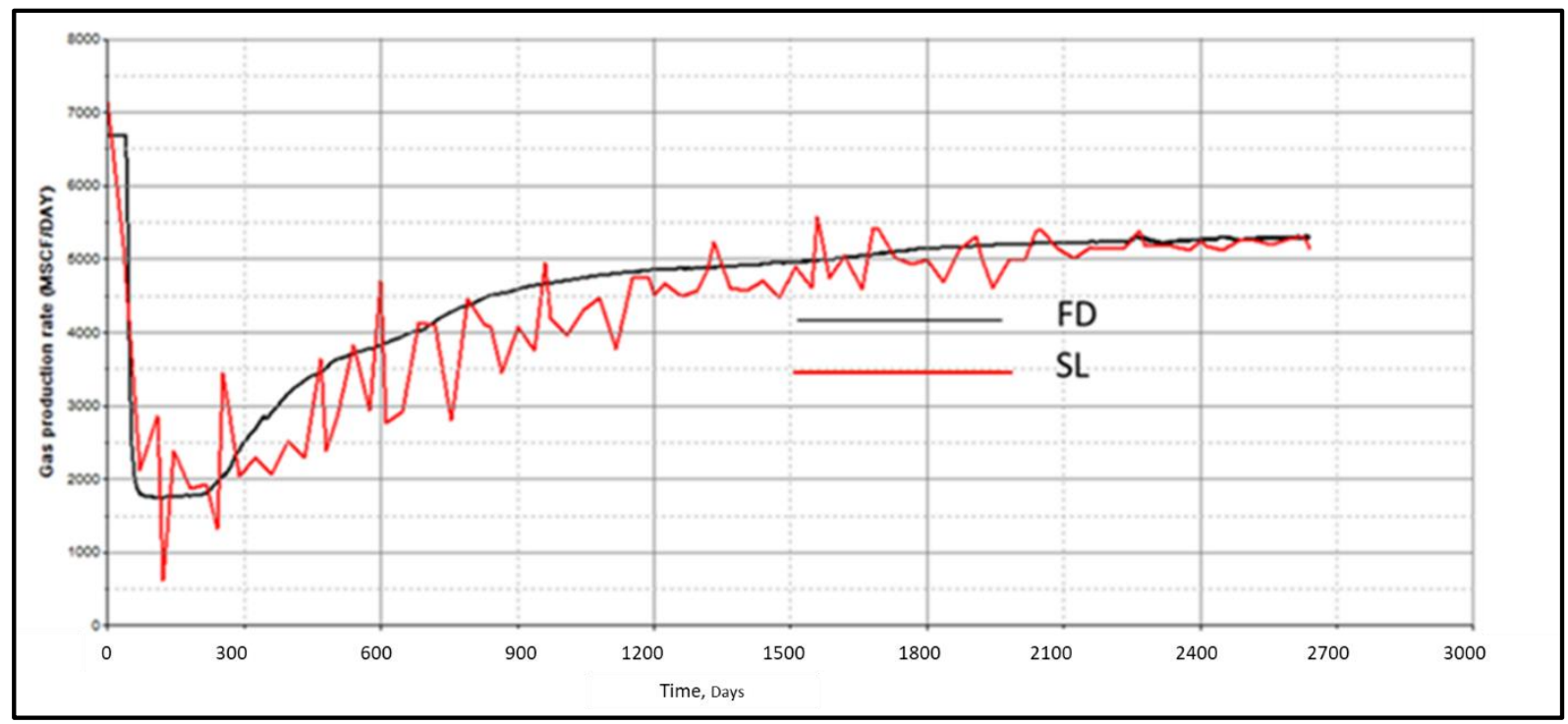

Figure 8-5: Gas Production rate for both FD and SL (Compositional)

For gas injection rates, Figure 8-6 presents gas injection rates calculated and observed in both FD and SL Models. As noticed from the plot, the gas injection rates of the SL model are always higher than those calculated by FD, and this is the main reason why the SL model has an over injection in terms of gas in comparison to FD which results in less oil production rate of the SL Model. 


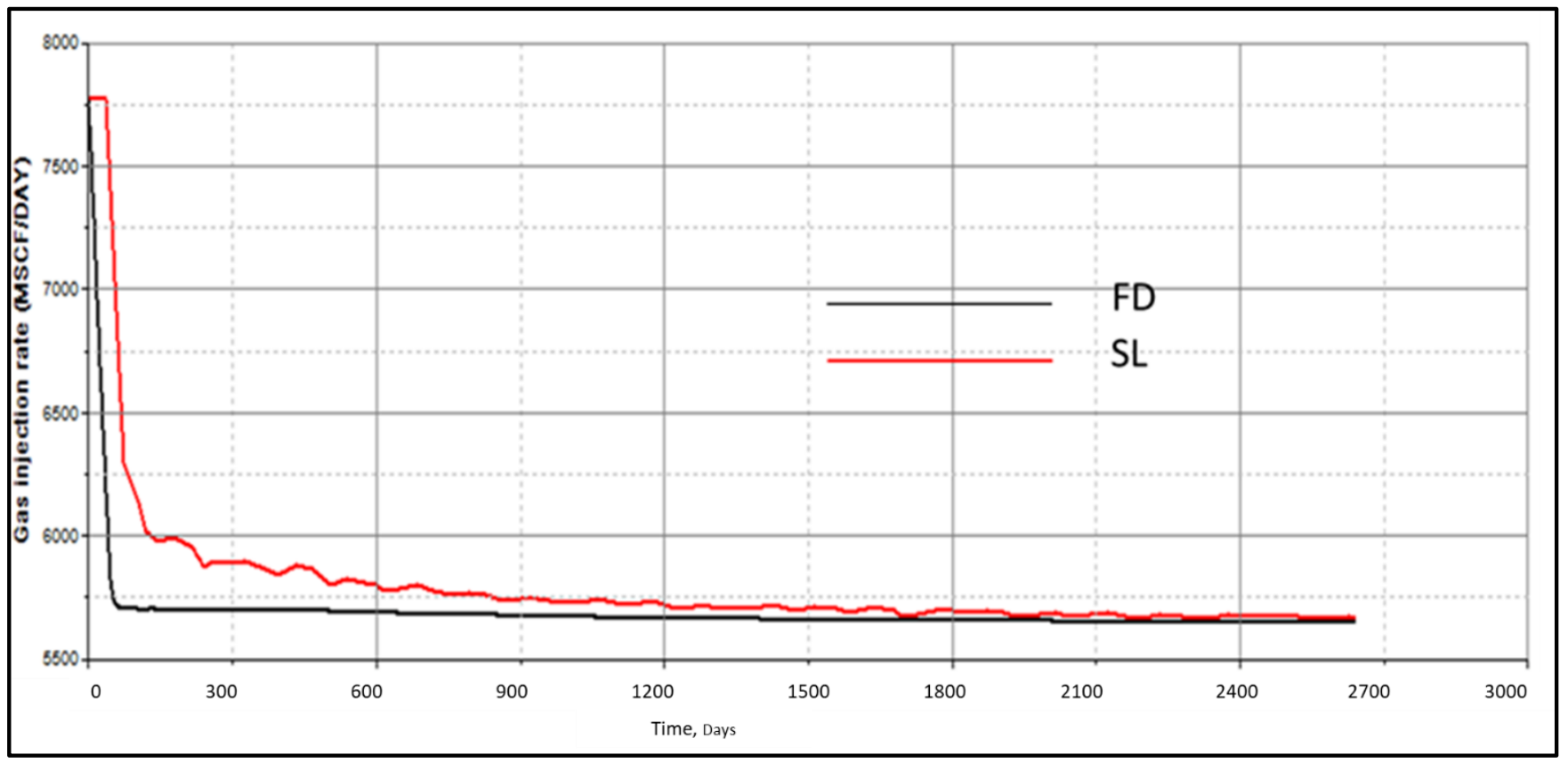

Figure 8-6: Gas Injection Rate for both FD and SL (Compositional).

For total CPU, Figure 8-7 presents the performance in both FD and SL cases. As can be seen from the plot for the case of the Fine Grid model, the FD model spent almost double the amount of total CPU used by SL.

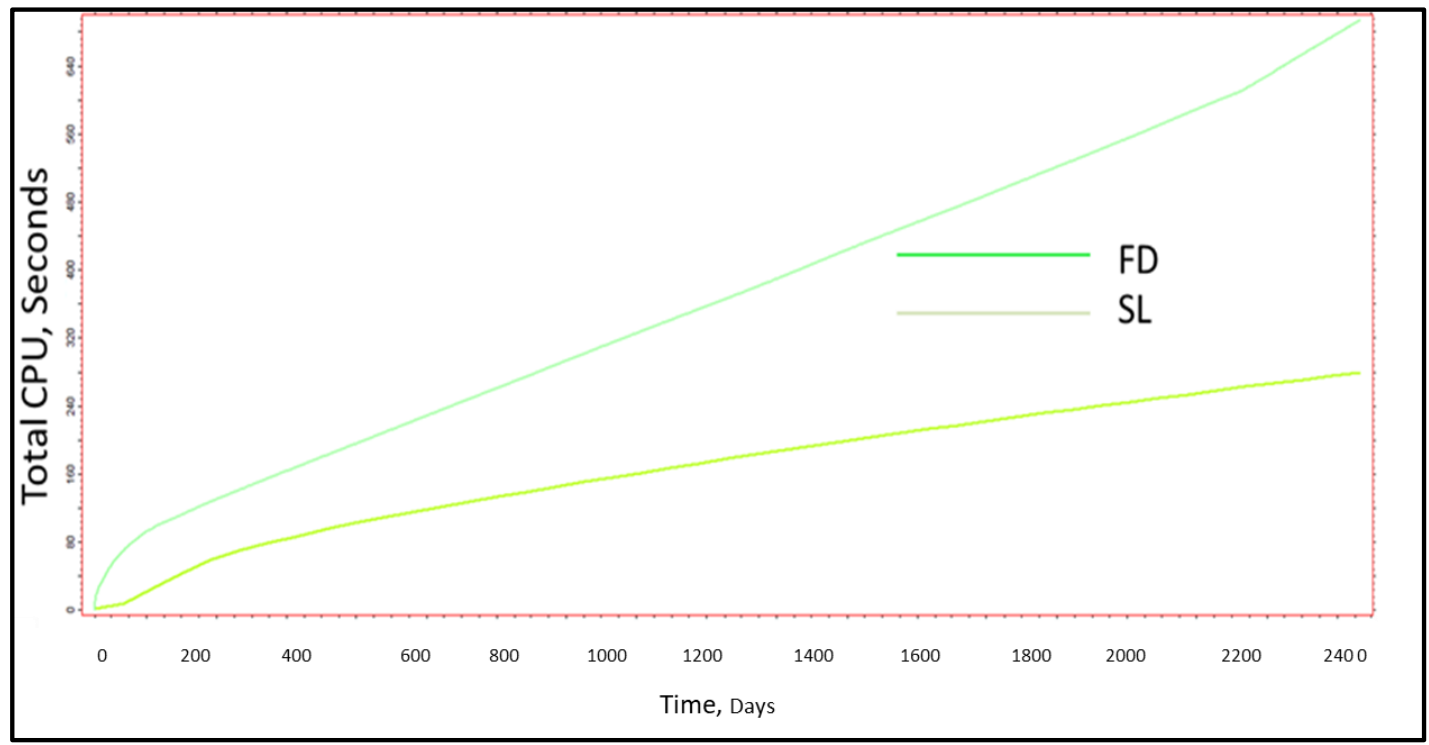

Figure 8-7: Total CPU required for FD and SL (Compositional) 


\subsubsection{Analysis of Compositional model}

To evaluate and assess the compositional model, the same tool presented in the base case gas injection model is also used here to measure and calculate the relative error. Figure 8-8 shows the relative error of oil rate for both FD and SL models. As can be seen from the plot, the relative error is fluctuating up and down and the average error is $+/-2 \%$.

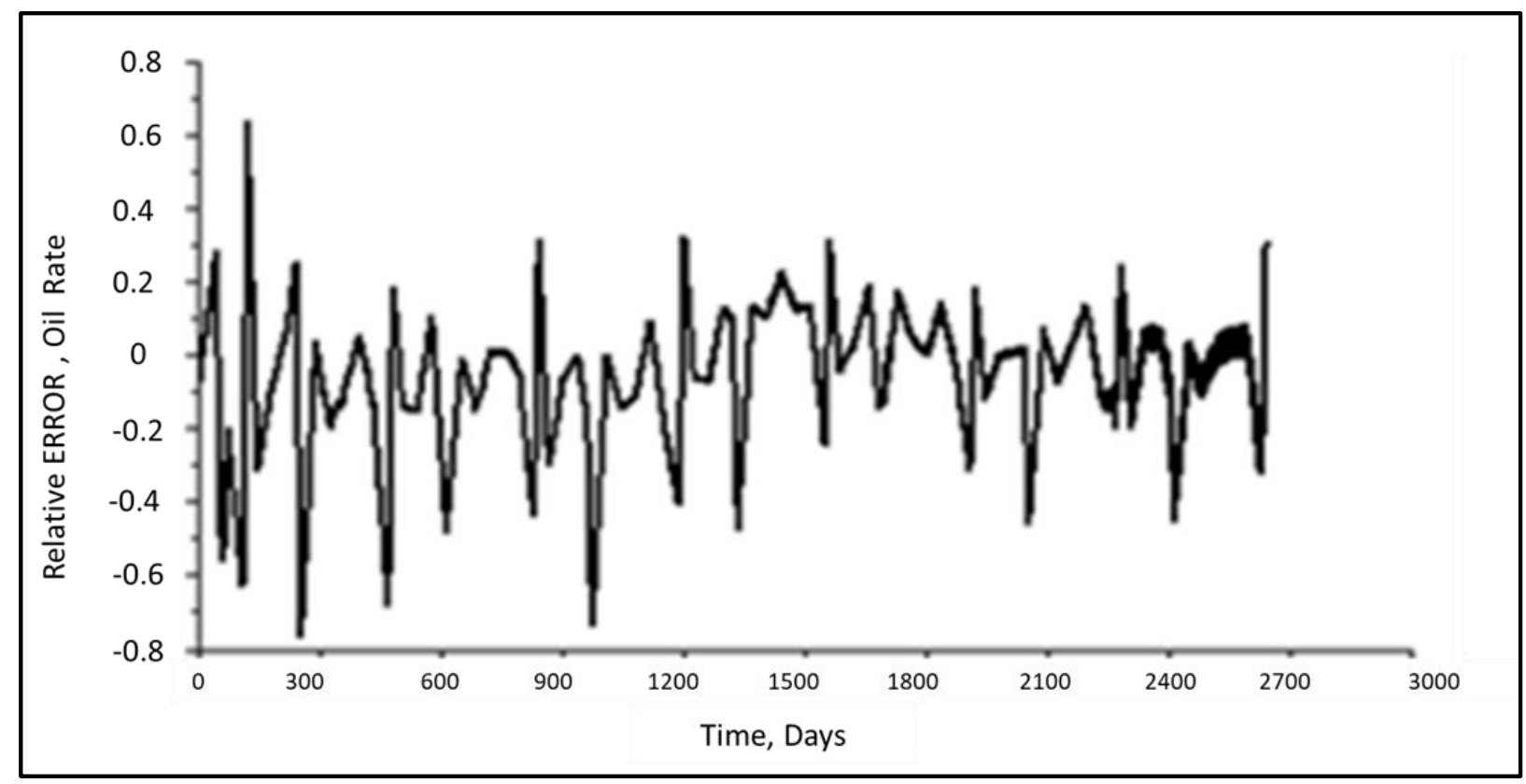

Figure 8-8: Relative Error Differences in Oil Production Rate between FD and SL Simulation cases (Compositional).

The relative errors of the cumulative oil production, as shown in Figure 8-9, are $+/-2 \%$. 


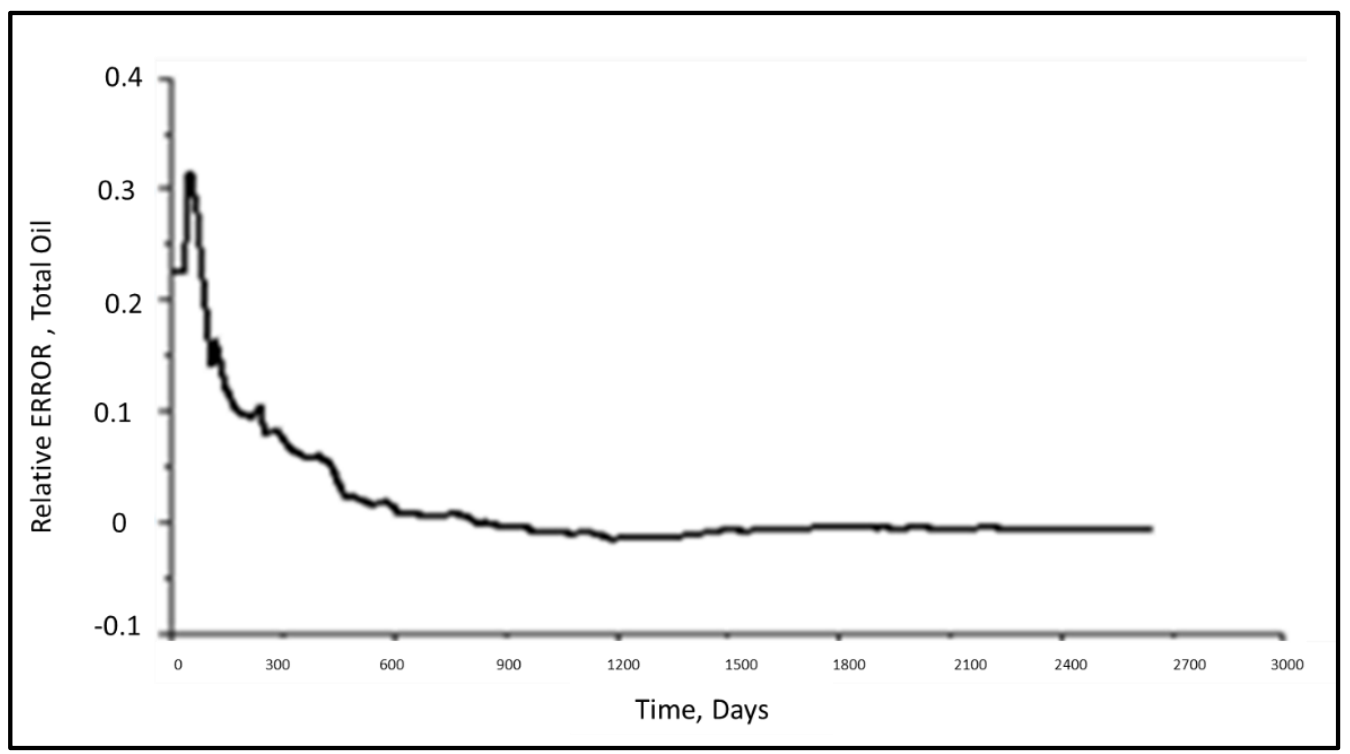

Figure 8-9: Relative Error in total Oil Production between FD and SL Simulation cases (Compositional).

Figure 8-10 shows the relative error of the gas rate and, as can be seen, the relative error in this case is approximately $5 \%$.

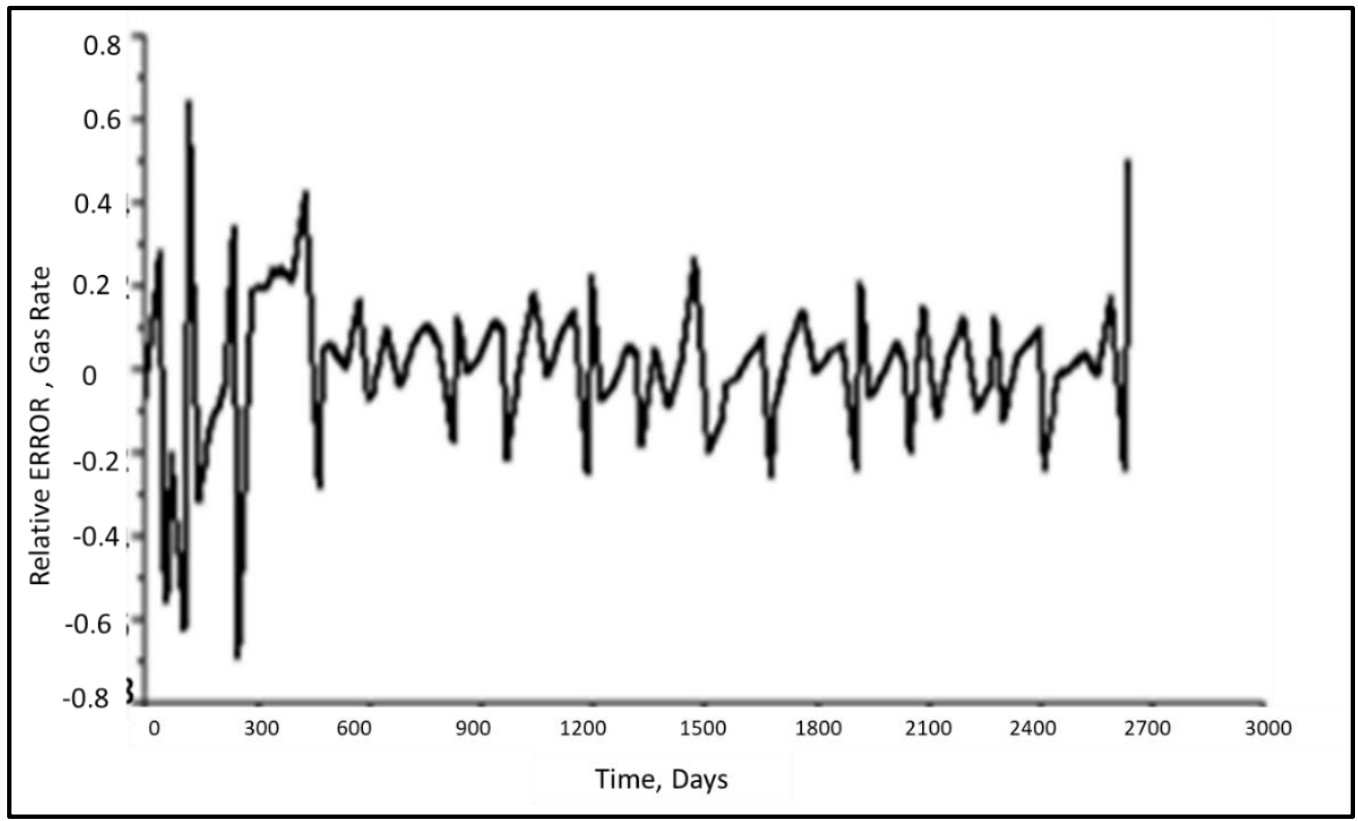

Figure 8-10: Relative Error in Gas Production between FD and SL Simulation cases (Compositional). 
Figure 8-11 shows the relative error of the gas injection and, as can be seen, the relative error in this case is approximately $1 \%$.

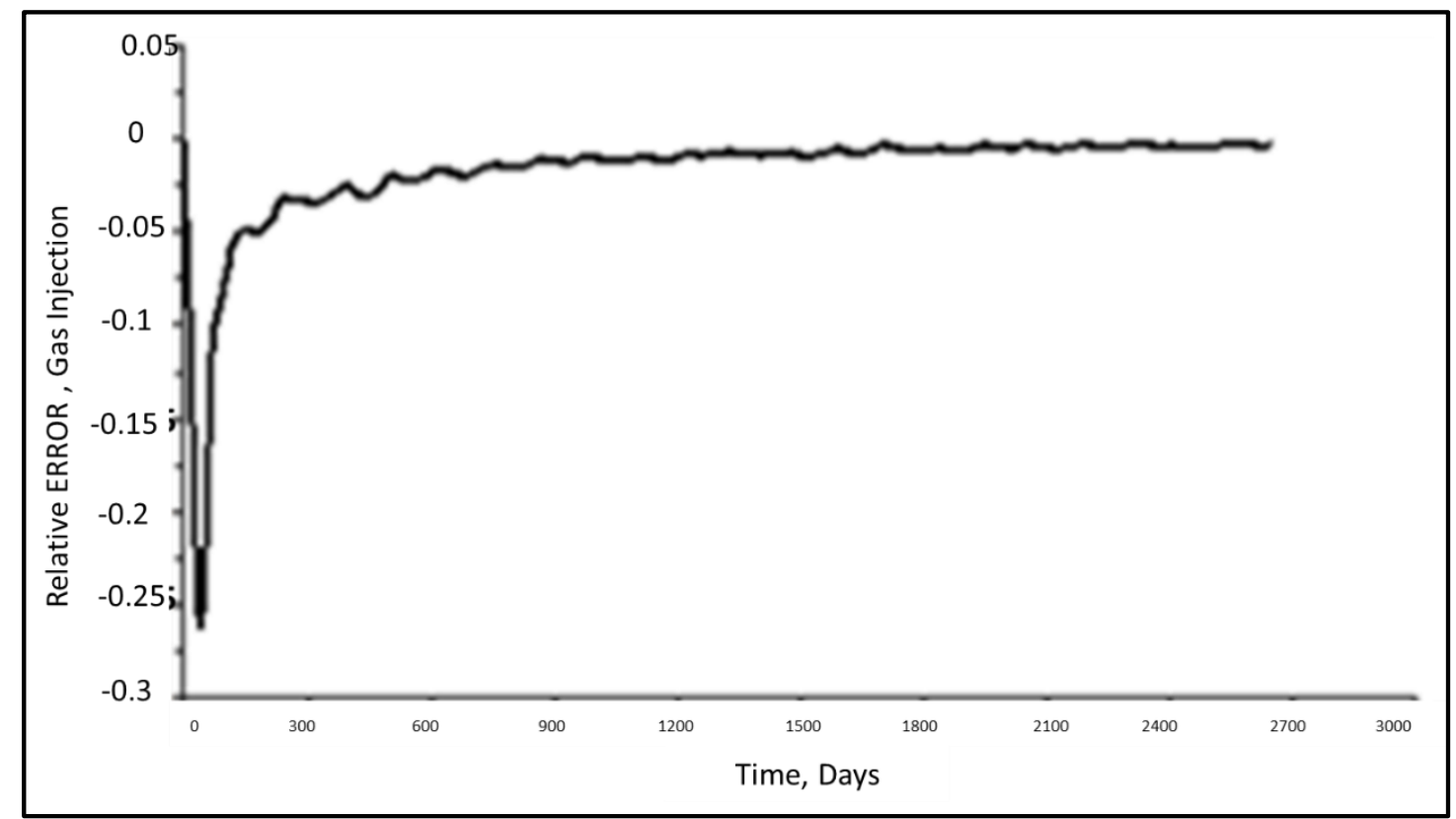

Figure 8-11: Relative Error on Gas Injection Rate between FD and SL simulation models (Compositional).

\subsubsection{FD VS SL 3D Analysis (Compositional Model)}

For the compositional models (FD Vs SL Simulation), figure 8-12 below shows the 3D oil saturation grid at the first-time step on the left and on the right shows at the end of simulation for both FD and SL compositional modelling case. As can be seen, similar trends are observed in both models at the first timestep of production (Left side). However, at the end of simulation minor changes are noticed (right side). 


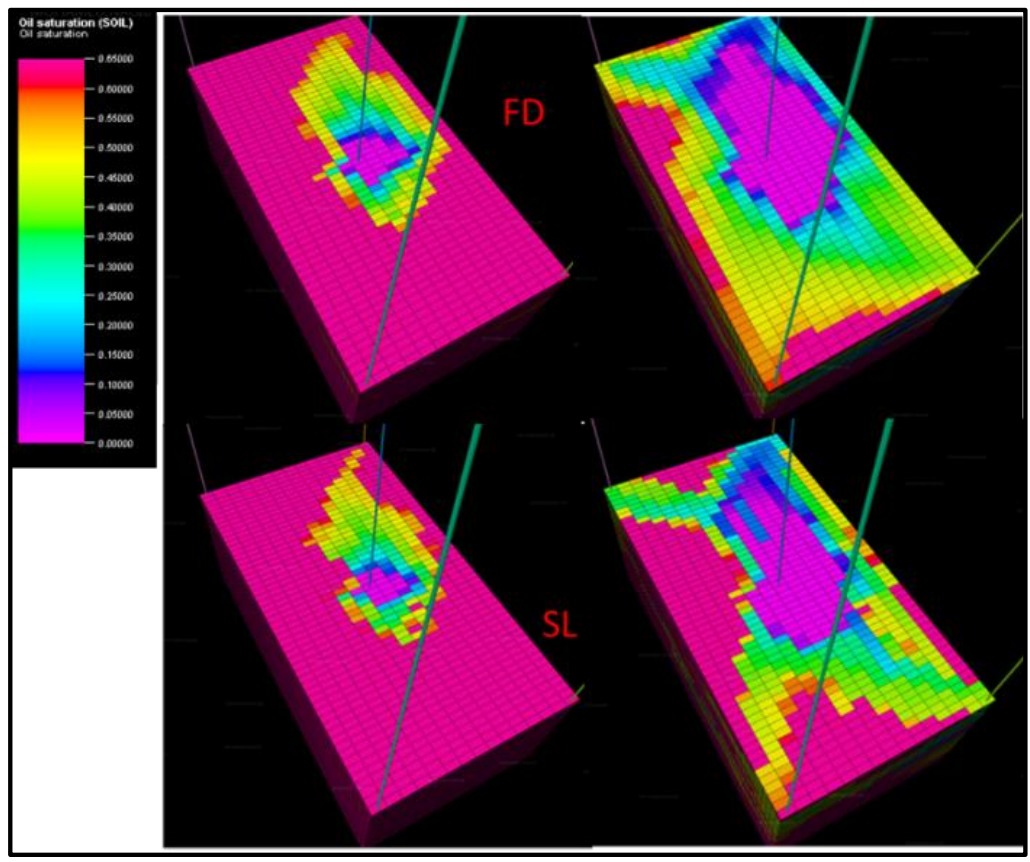

Figure 8-12: 3D Oil saturation Grid for Compositional Modelling at 1st and Last Time step for Both FD and SL.

Sliced 3D oil saturation grids for both FD and SL are presented in figures 8-13. Minor differences observed as well at the last timestep.

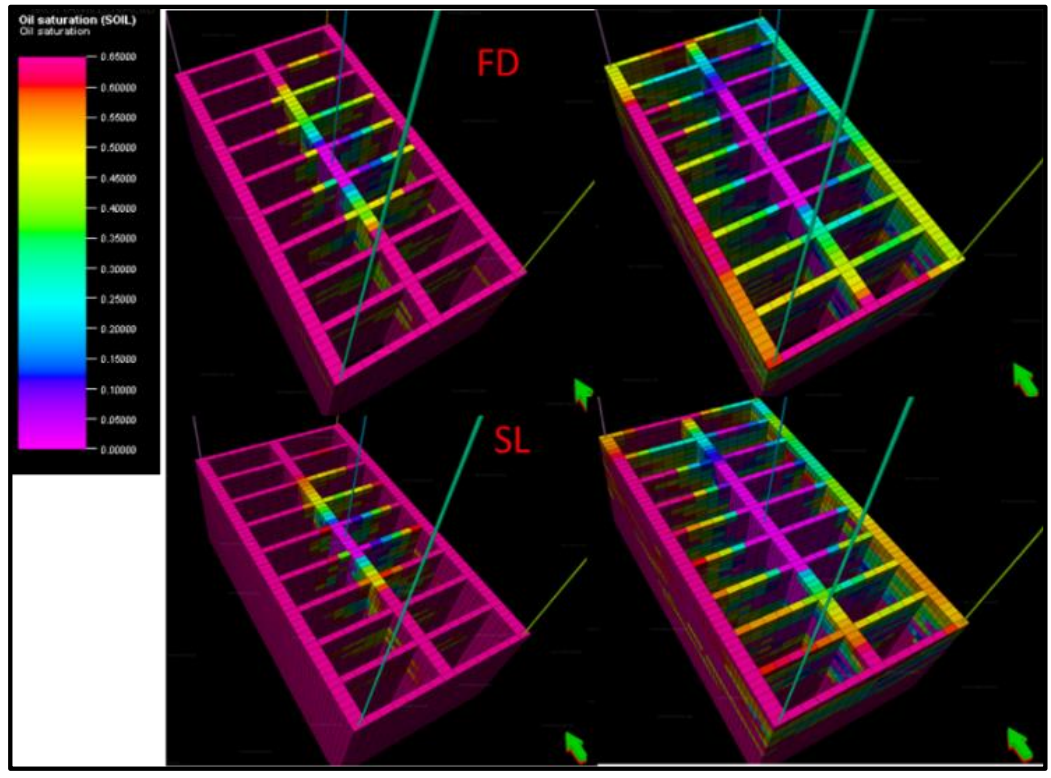

Figure 8-13: Sliced Oil saturation Grid for Compositional Modelling at 1st and Last Timestep for Both FD and SL. 
Similarly, oil saturation grid cross section in I direction for both FD and SL also presented in Figure 8-14. As can be noticed, similar trends with minor differences.

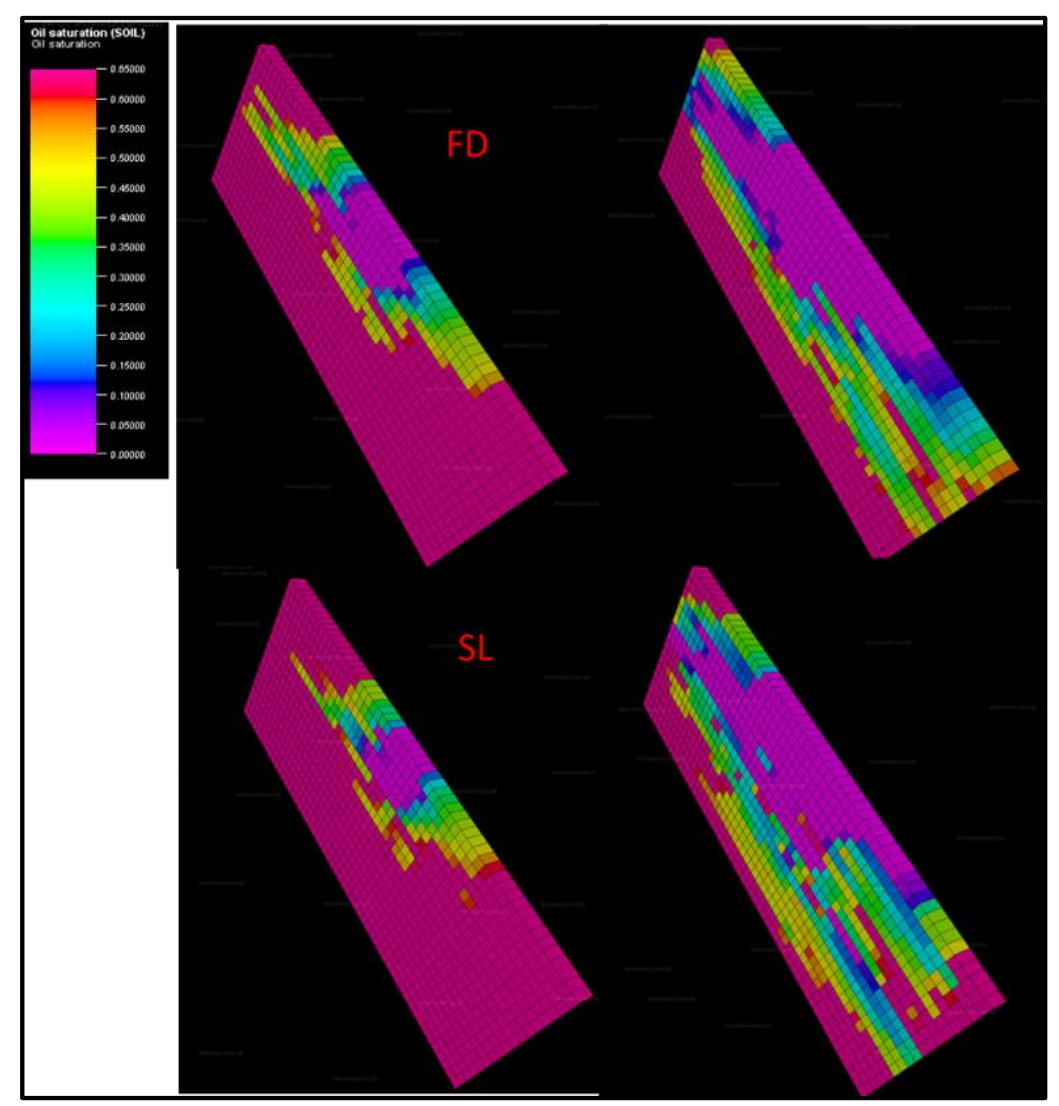

Figure 8-14: Oil saturation Grid cross section for Compositional Model at 1st and Last Time Step.

Similar analysis done as well for gas saturation. FD and SL 3D gas Saturations at first- and last-time steps is presented in figure 8-15. 


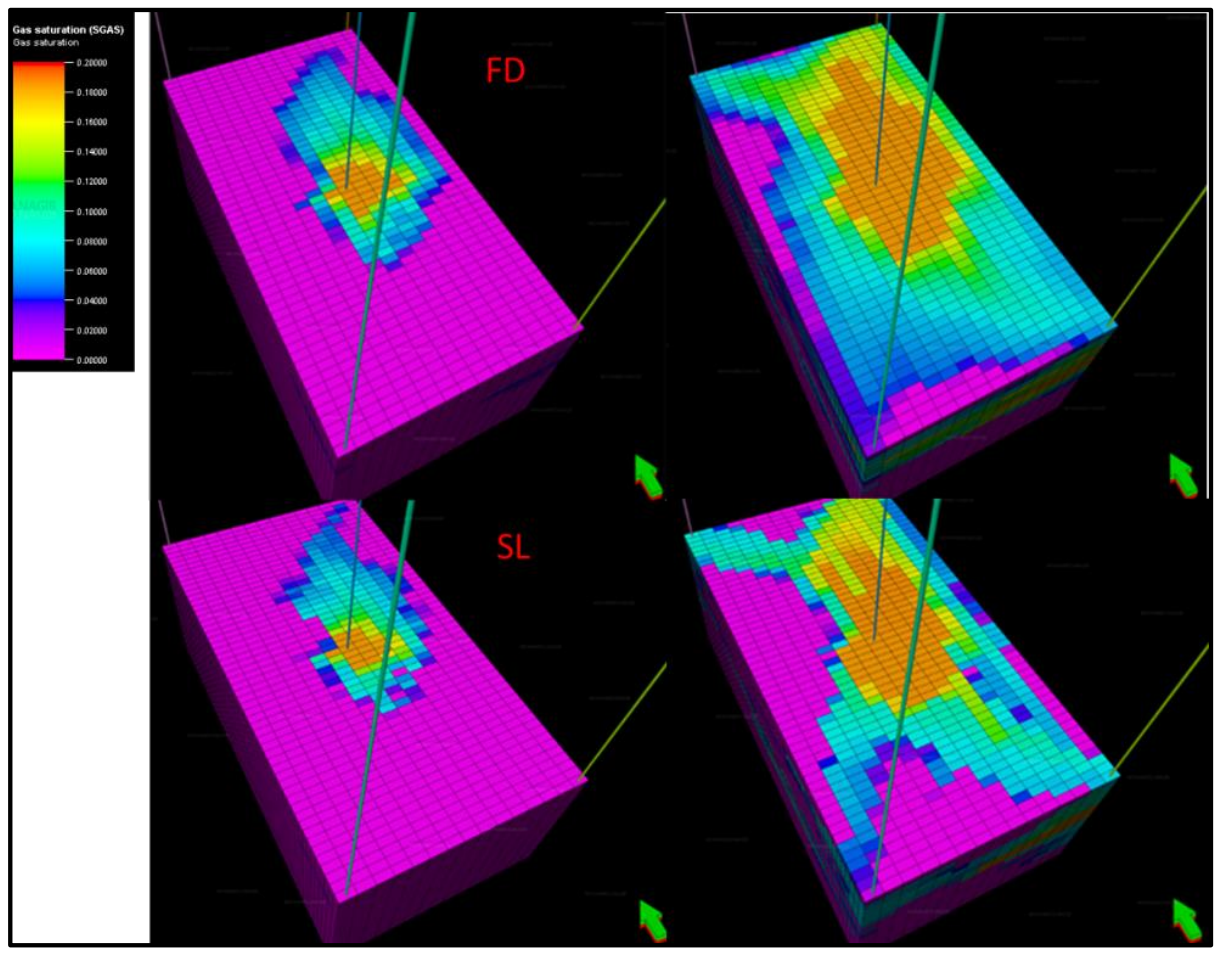

Figure 8-15: 3D Gas Saturation Grid for Compositional Modelling at $1^{\text {st }}$ and Last Timestep for Both FD and SL.

As can be seen from the graph above, similar trends are observed in both models with some minor differences. Sliced 3D Gas saturation grids for both FD and SL are presented in figures 8-16. Two models showed minor differences in terms of gas saturation grid. 


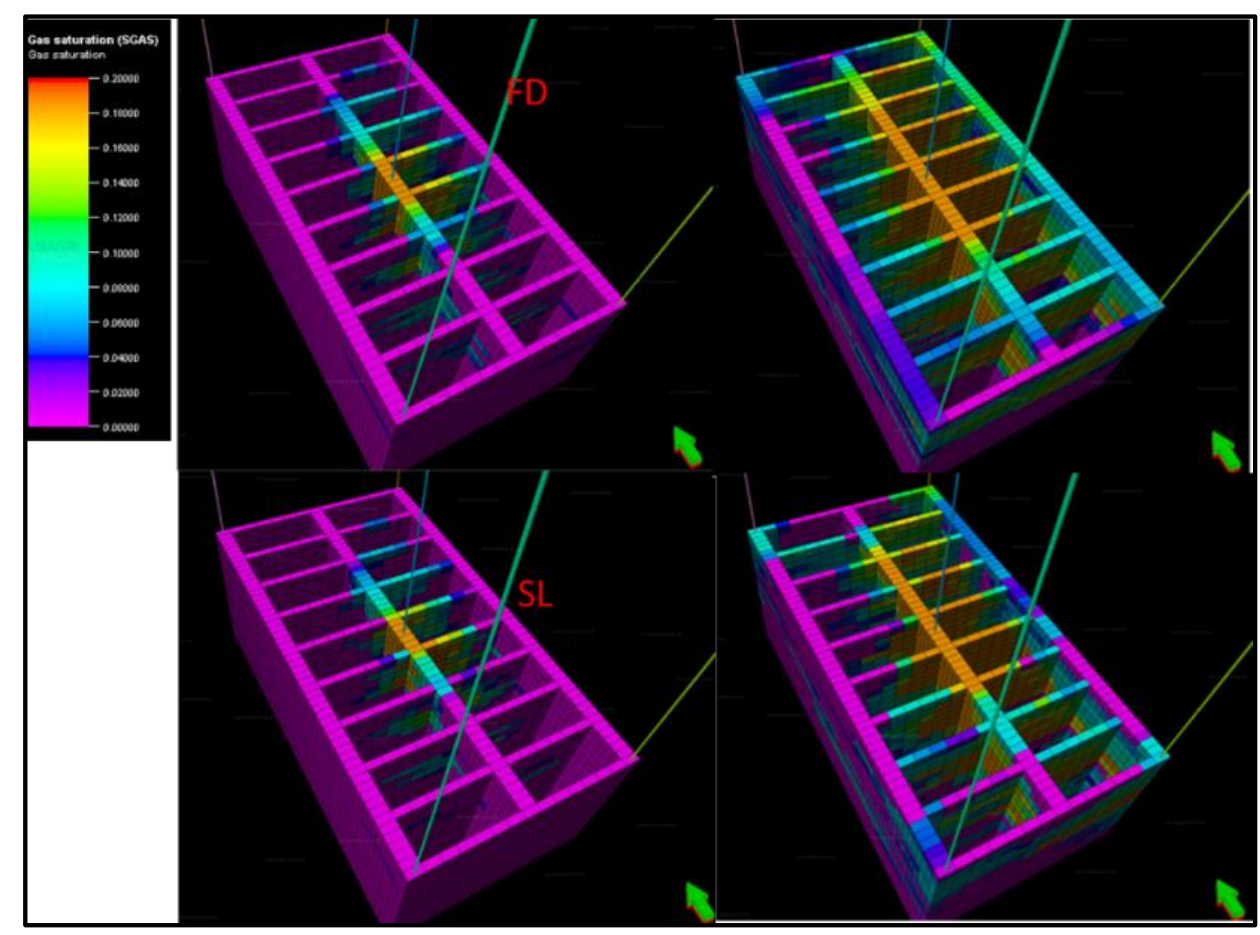

Figure 8-16: Sliced Gas saturation Grid for Compositional Modelling at $1^{\text {st }}$ and Last Timestep for Both FD and SL.

Gas saturation grid cross section in I direction for both FD and SL are presented in Figure 8-17. Similar trends also observed here with minor differences. 


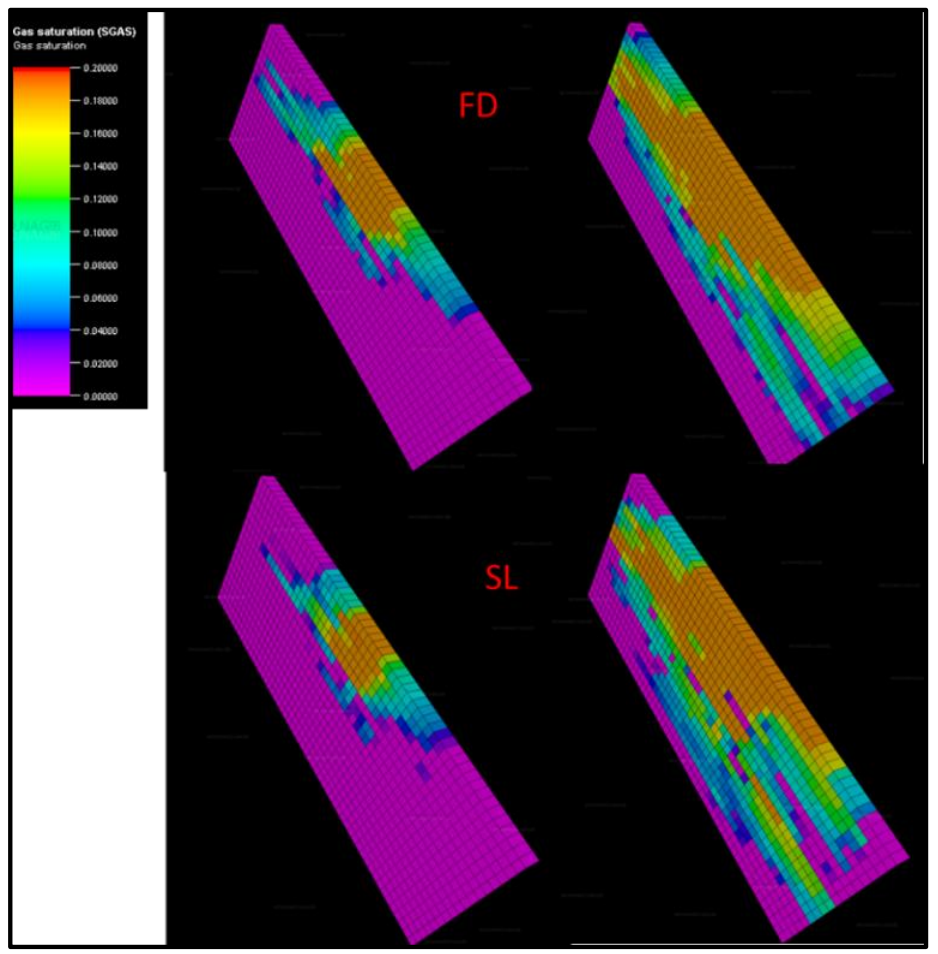

Figure 8-17: Gas Saturation Grid cross section for Compositional Model at $1^{\text {st }}$ and Last Timestep.

Figure 8-18 shows Compositional model OTC at the end of field life of both FD and SL models.

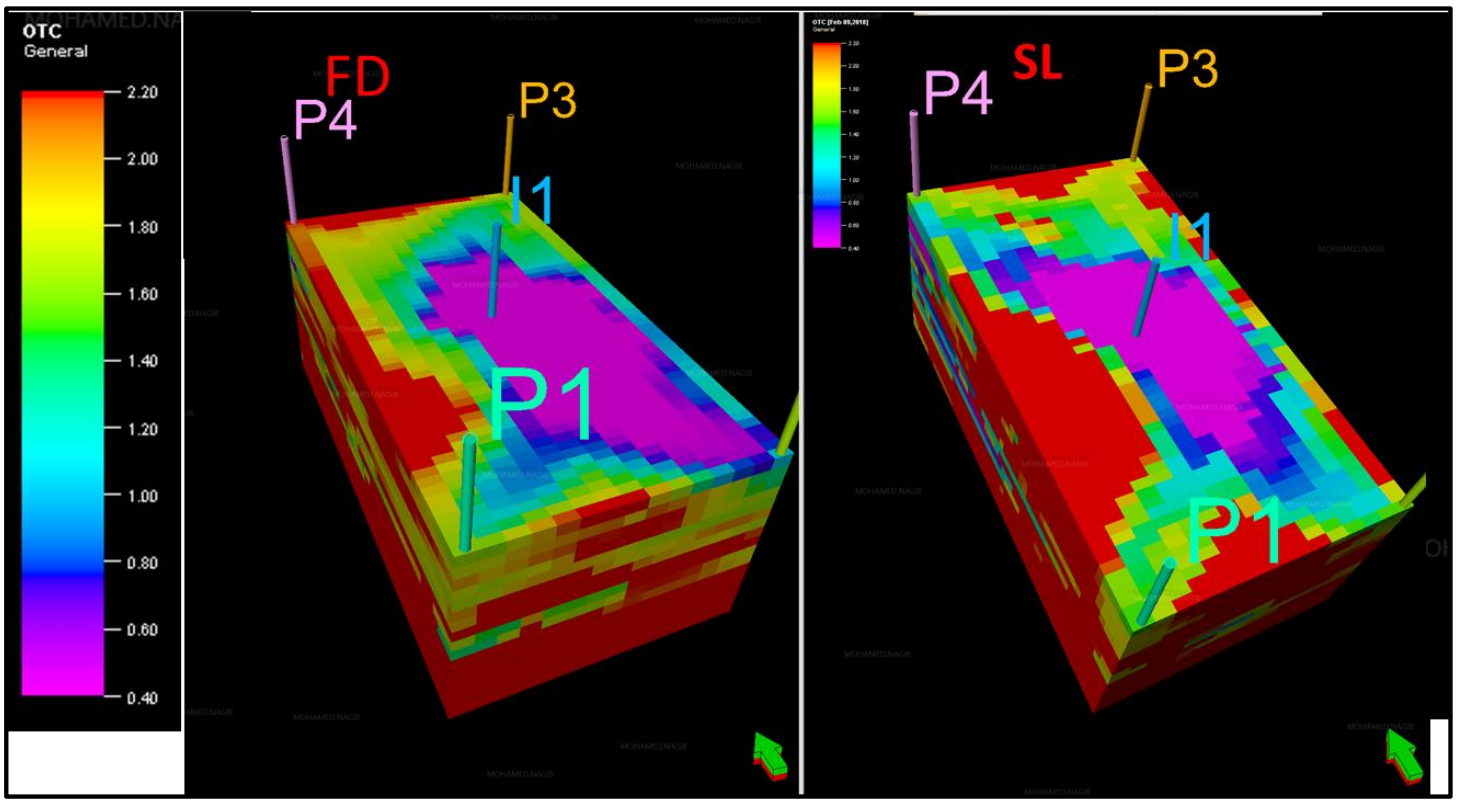

Figure 8-18: Compositional model OTC at the end of field life of both FD and SL models 
From all the above analysis it is concluded that commotional modelling simulation gives similar results as FD compositional analysis with the major benefits of CPU time saving

\subsection{Miscible Gas Injection}

The miscible Enhanced Oil Recovery (EOR) process involves the use of supercritical $\mathrm{CO}_{2}$ to displace the oil from a depleted oil reservoir with suitable characteristics, typically light oil. The miscible flood model is simply an implementation of empirical treatment. In this case four-components of the black oil model are provided for modelling reservoir recovery mechanisms of which injected fluids are miscible with hydrocarbons in the reservoir to model gas injection without going to the complexity of compositional modelling. The solvent extension implements the Todd and Longstaff empirical model for miscible floods [79].

In miscible gas injection processes, simulation of incompressible miscible displacement was initially based on a direct solution of the convection-diffusion equations for the local concentration of each of the miscible components. The numerical diffusion inherent in finite difference models also masks the true mixing of the miscible components unless the grid is sufficiently fine. Typically the miscible injection fluid is expensive compared with traditional flooding fluids, such as dry gas and water. Miscible fluids are often referred to as 'solvents'; examples of solvent schemes are listed below:

- High pressure dry gas process

- A solvent as LPG or Propane could be inject as slug followed by lean gas injection.

- Non hydrocarbon gases as $\mathrm{CO}_{2}$ produce miscible displacement of oil

The injected miscible $\mathrm{CO}_{2}$ mixes thoroughly with the oil within the reservoir such that the interfacial tension between these two substances disappears. The $\mathrm{CO}_{2}$ has the ability to dissolve in, swell and then reduce the viscosity of oil.

In our case, PVT and relative permeability data was replaced by a miscible gas injection set of data and then tested on the model. Figure 8-19 presents the new relative permeability data set. 


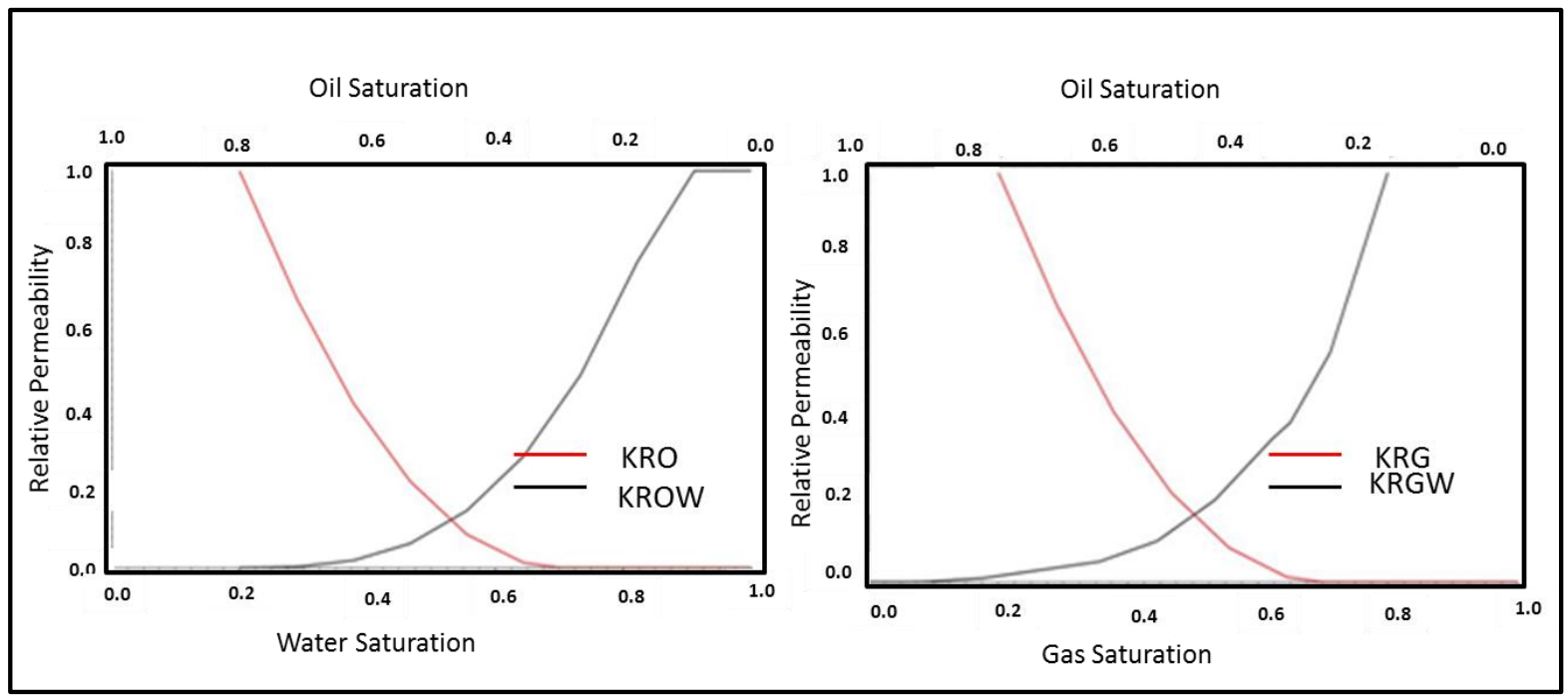

Figure 8-19: Miscible gas injection relative permeability data used in both FD and SL simulations

For the oil production rate, Figure 8-20 presents the oil production rate forecasted in both FD and SL cases. The figures illustrate the field oil production calculated by SL (red colour) and the same calculated by FD (black colour). As can be seen in the plots, both models have the same initial oil rate. However, the decline rate of the SL model is gentler in comparison to the FD model, and this will result in a slight mismatch in the total oil production.

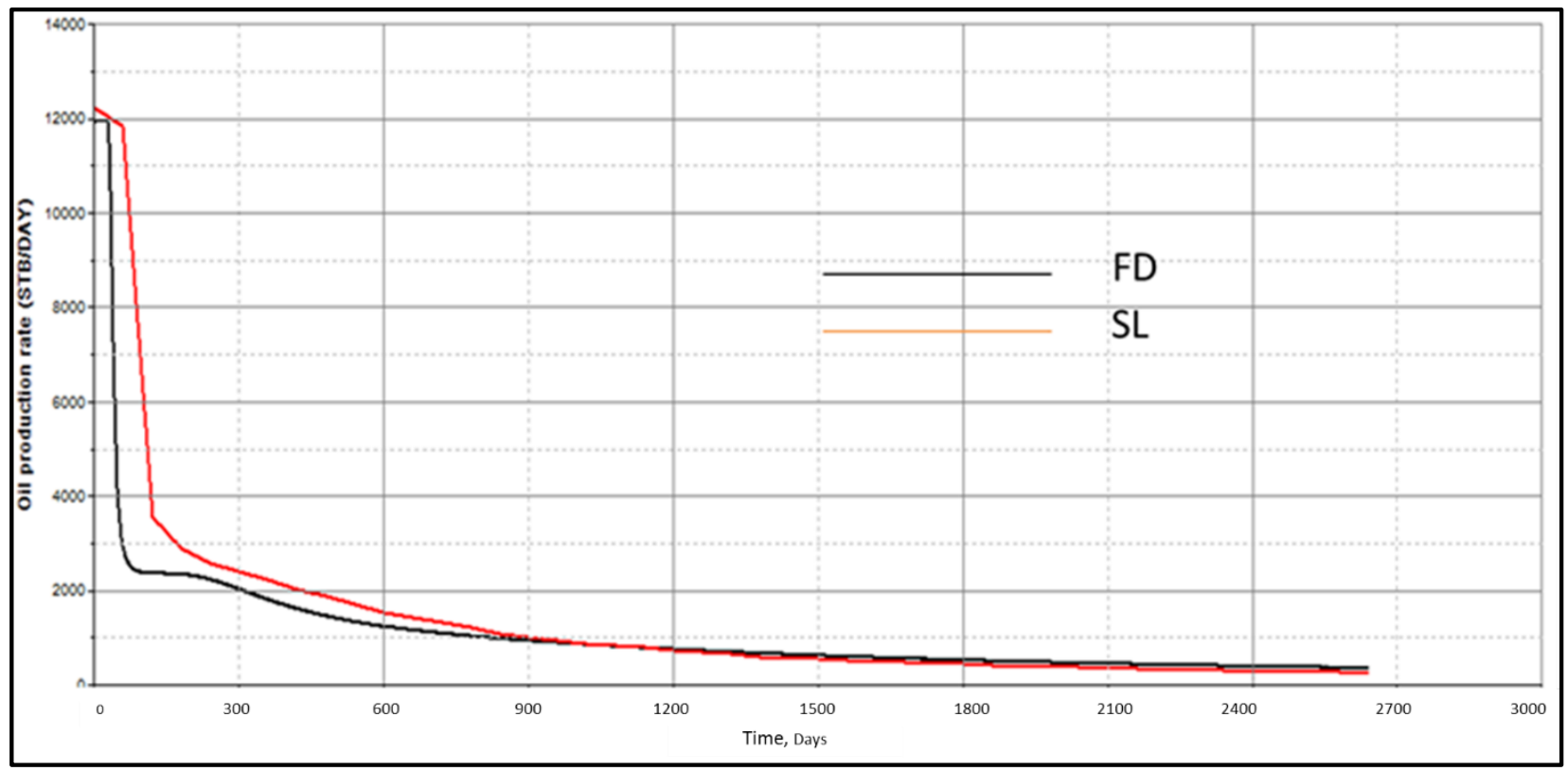

Figure 8-20: Oil production Rates for both FD and SL (Miscible Gas) 
For cumulative oil production, Figure 8-21 presents cumulative oil production forecasted in both FD and SL cases. As can be seen from the plot, the total oil production calculated by SL is slightly higher in comparison to the FD model, and this mainly results from the mismatch of the oil rate presented earlier.

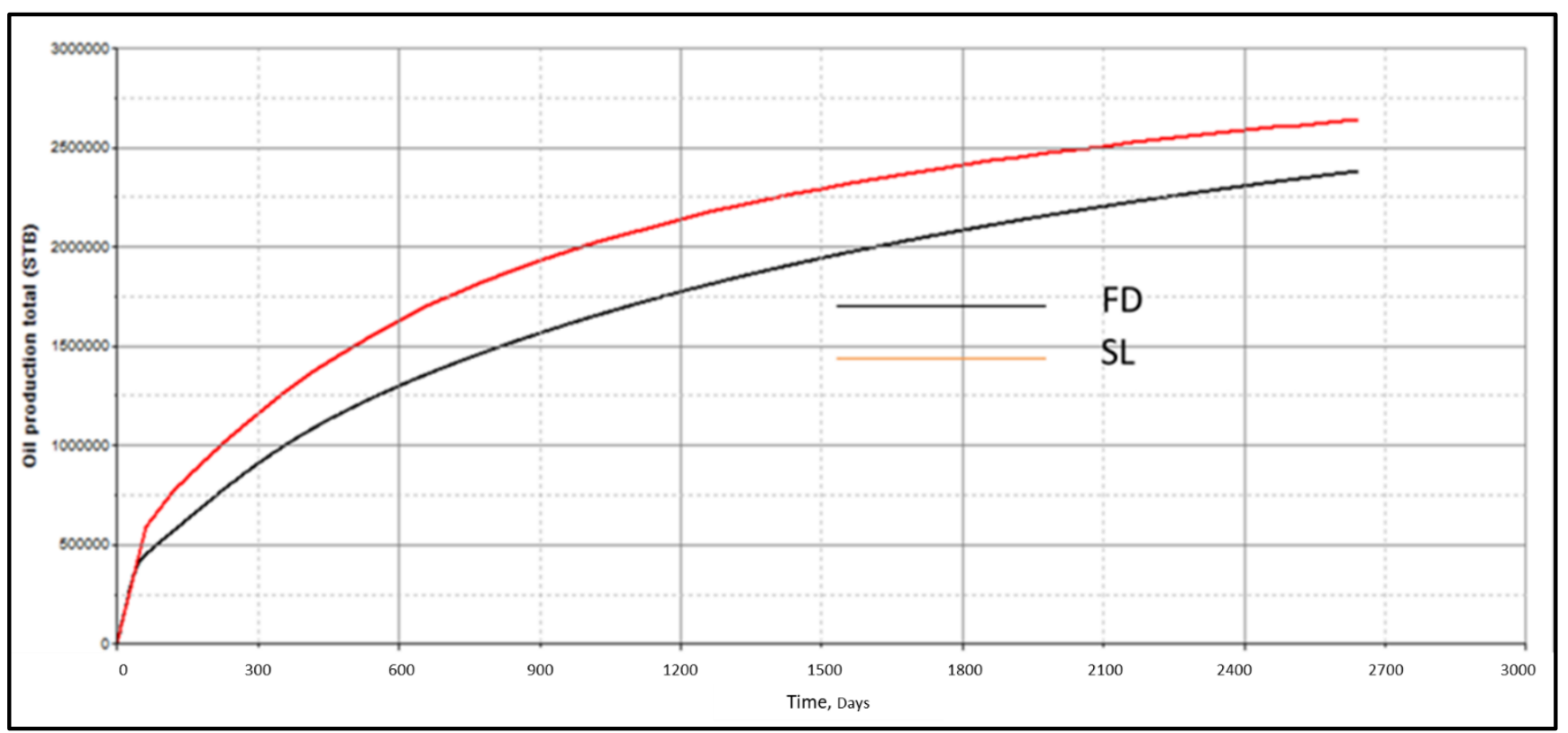

Figure 8-21: Cumulative Oil production for both FD and SL (Miscible Gas)

Figure 8-22 below presents gas production rates calculated in both FD and SL models. As can be seen from the plot, both trends are similar. However, the initial gas production predicted by SL is slightly lower in comparison to the one predicted by FD, and the overall trends are matching. 


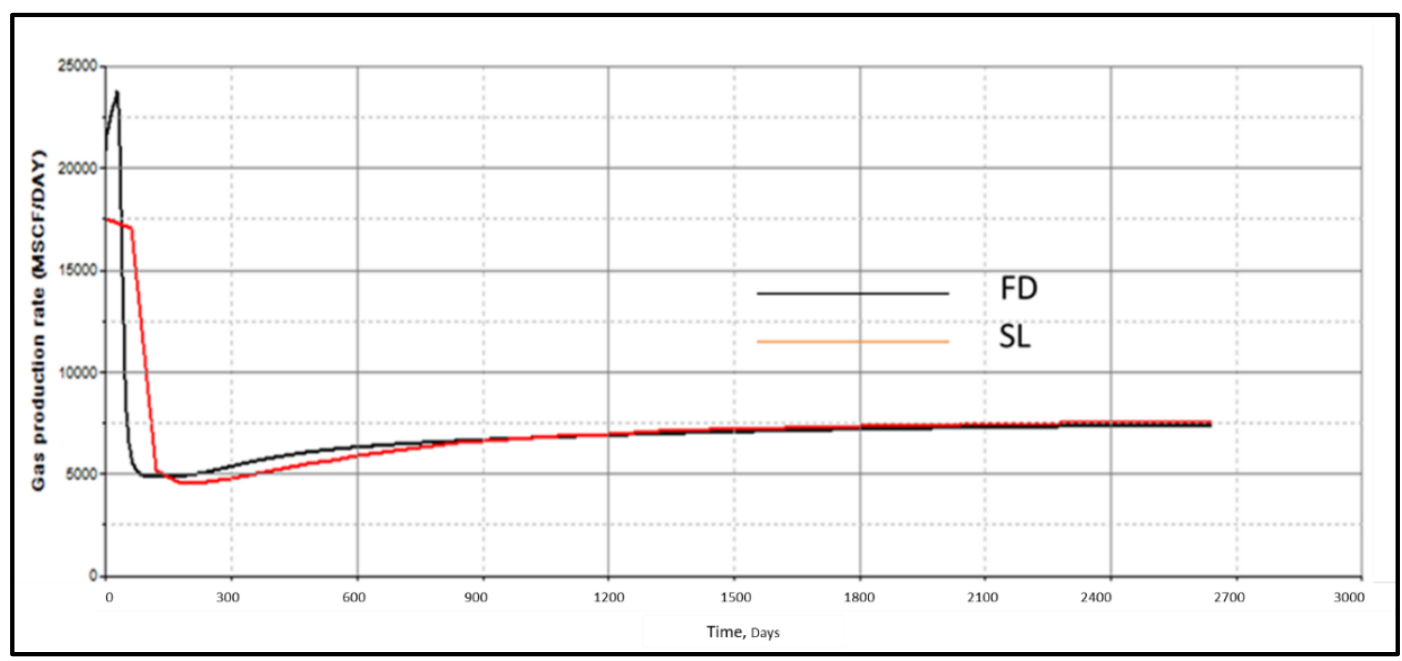

Figure 8-22: Gas Production rates for both FD and SL (Miscible Gas)

For gas injection rates, Figure 8-23 presents gas injection rates calculated and observed in both FD and SL Models. As noticed from the plot, apart from the early time period, both models are in good agreement.

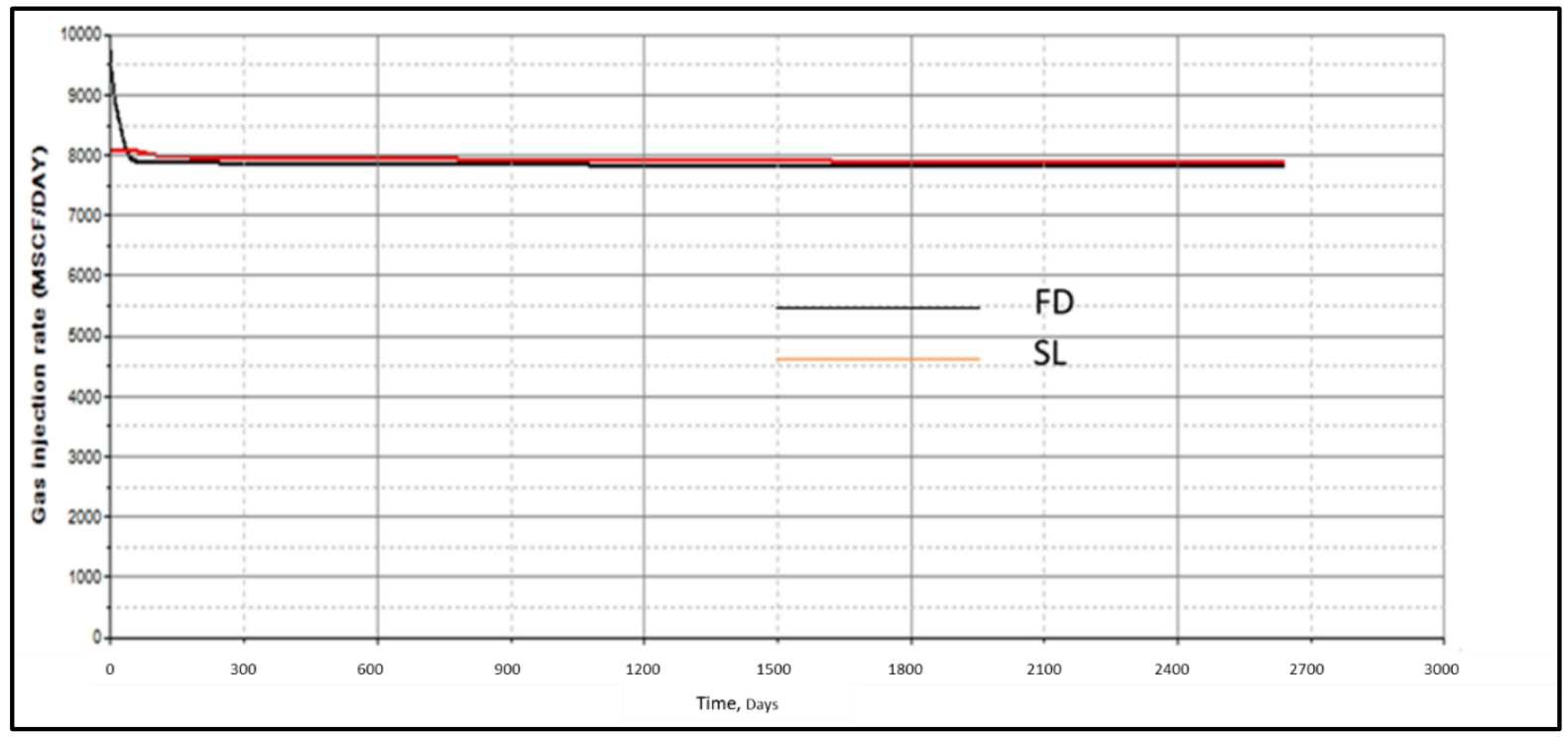

Figure 8-23: Gas Injection Rate for both FD and SL (Miscible Gas).

Reservoir pressure performance in both FD and SL cases are displayed below in figure 8-24. As can be seen from the plot, both trends are similar. However, the pressure in SL simulation is slightly lower in comparison to the FD simulation, and this is generally acceptable as the pressure field is solved in the conventional finite difference manner and streamlines are traced from injector to producer. 


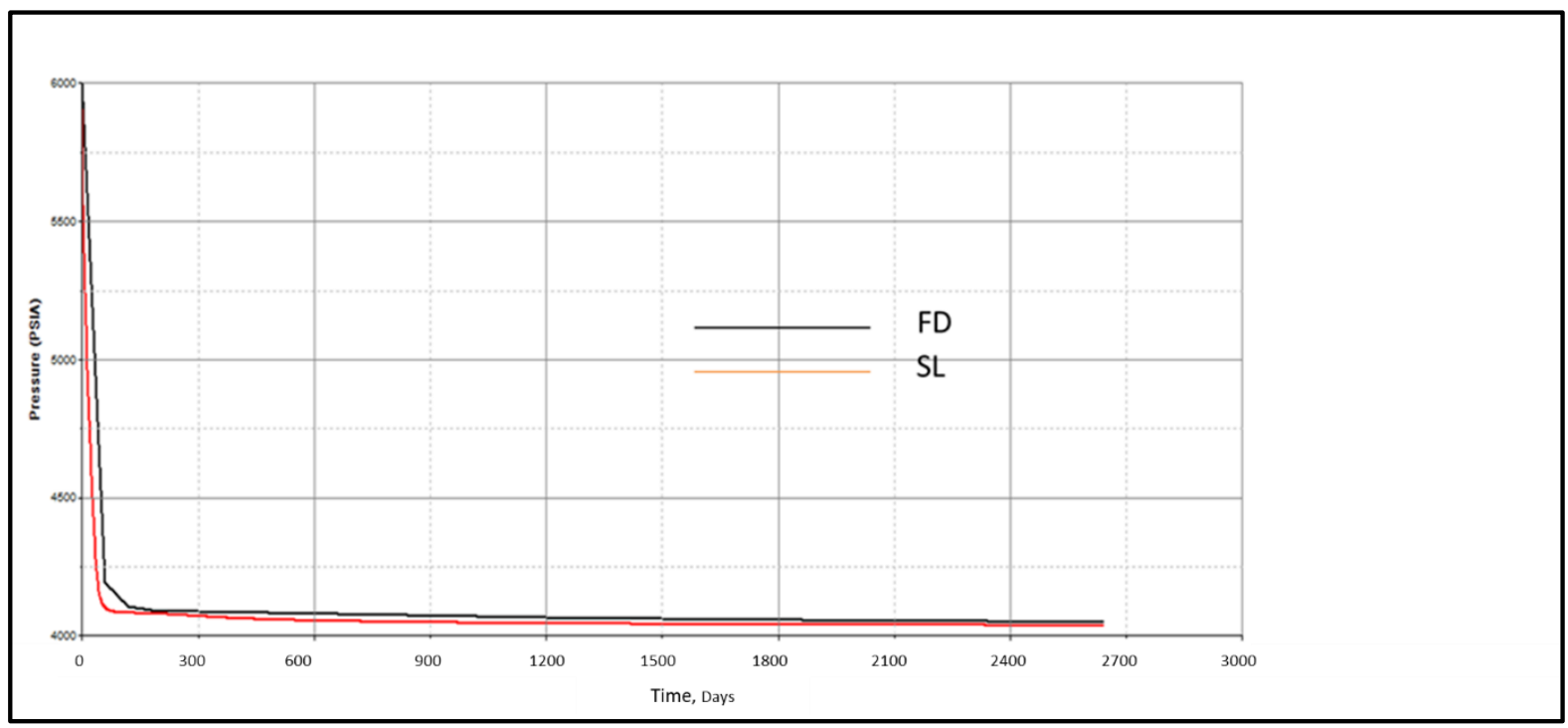

Figure 8-24: Reservoir Pressure Performance for both FD and SL (Miscible Gas).

For total CPU, Figure 8-25 presents the performance in both FD and SL cases. As can be seen from the plot for the case of Fine Grid model, the FD model spent almost doubles the total CPU time used by SL.

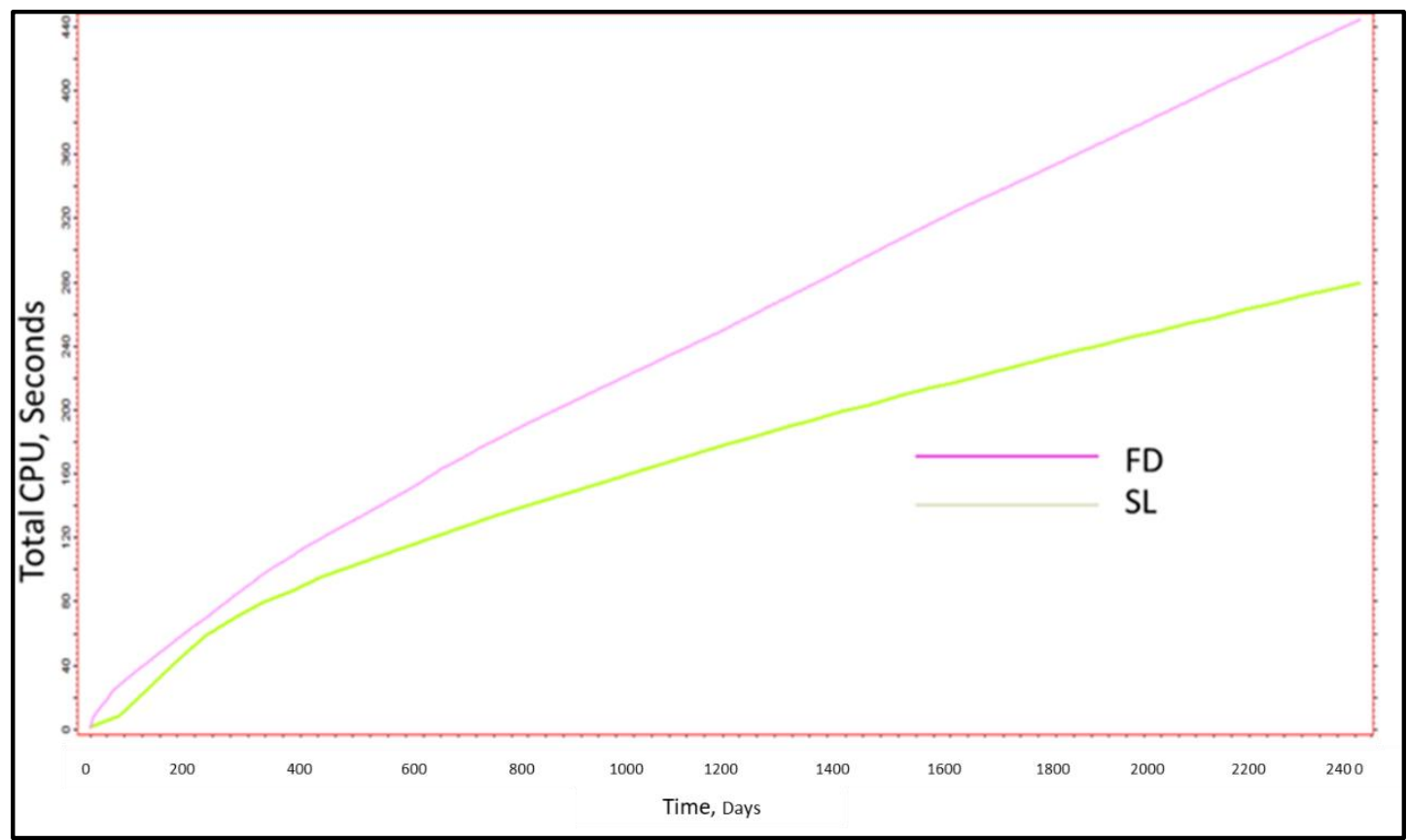

Figure 8-25: Total CPU required for FD and SL (Miscible Gas) 


\subsection{Analysis of Miscible Flood Model}

To evaluate and assess the miscible flood model, the same tool presented above is used. Figure 8-26 shows the relative error of oil rate for both FD and SL models. As can be seen from the plot, the relative error is the least amongst the other models, approximately equal to $-3 \%$.

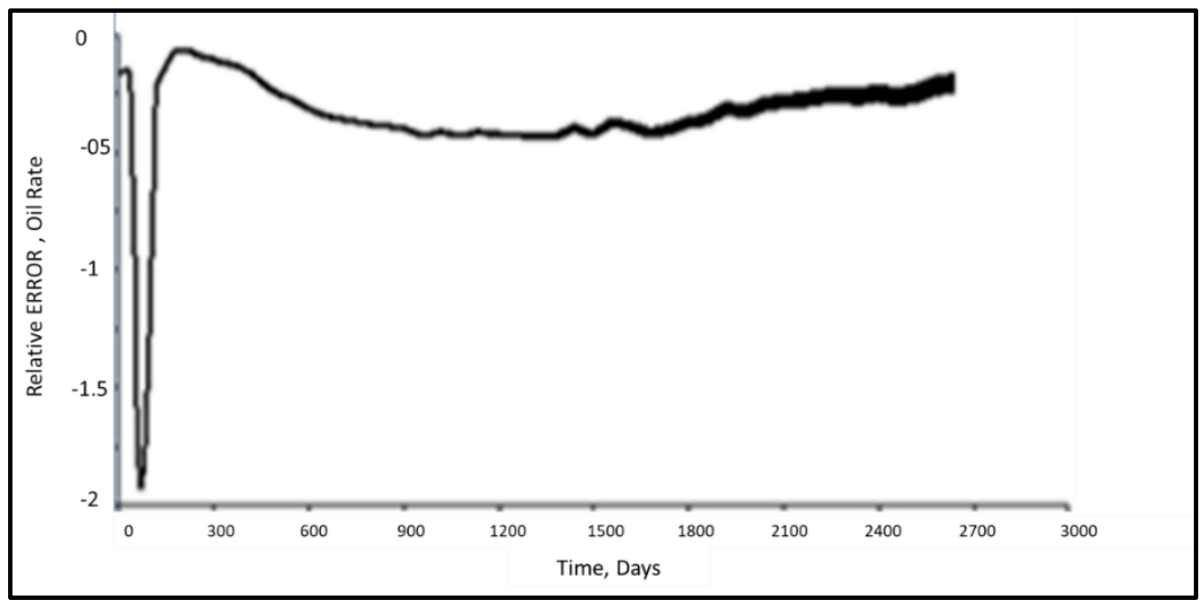

Figure 8-26: Relative Error in Oil Production Rate between FD and SL Simulation cases (Miscible Gas).

The relative error differences for cumulative oil production are shown in Figure 8-27. The relative error in this case is approximately $-5 \%$

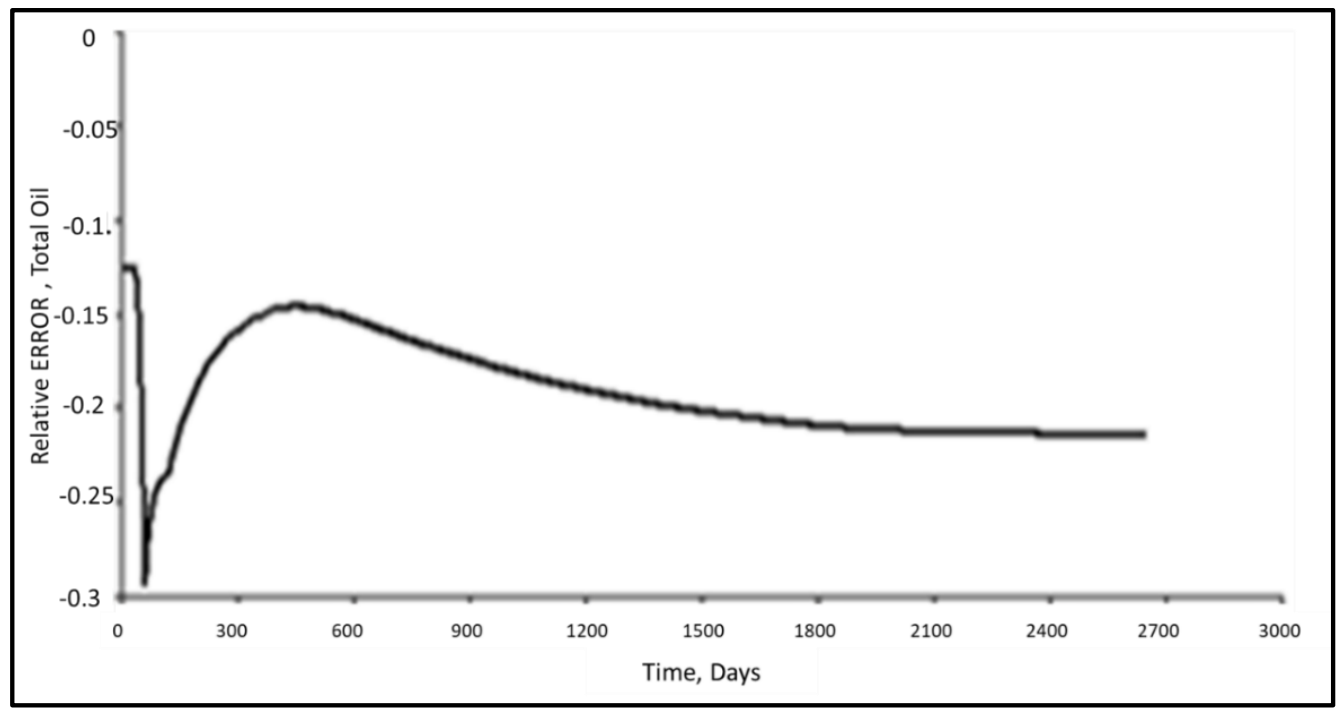

Figure 8-27: Relative Error in Cumulative Oil Production between FD and SL Simulation cases (Miscible Gas). 
Figure 8-28 shows the relative error of the gas rate and, as shown in the graph, initially this error is relatively high as it is reaches $-20 \%$ for a short period, then flattens towards the end with almost no relative error.

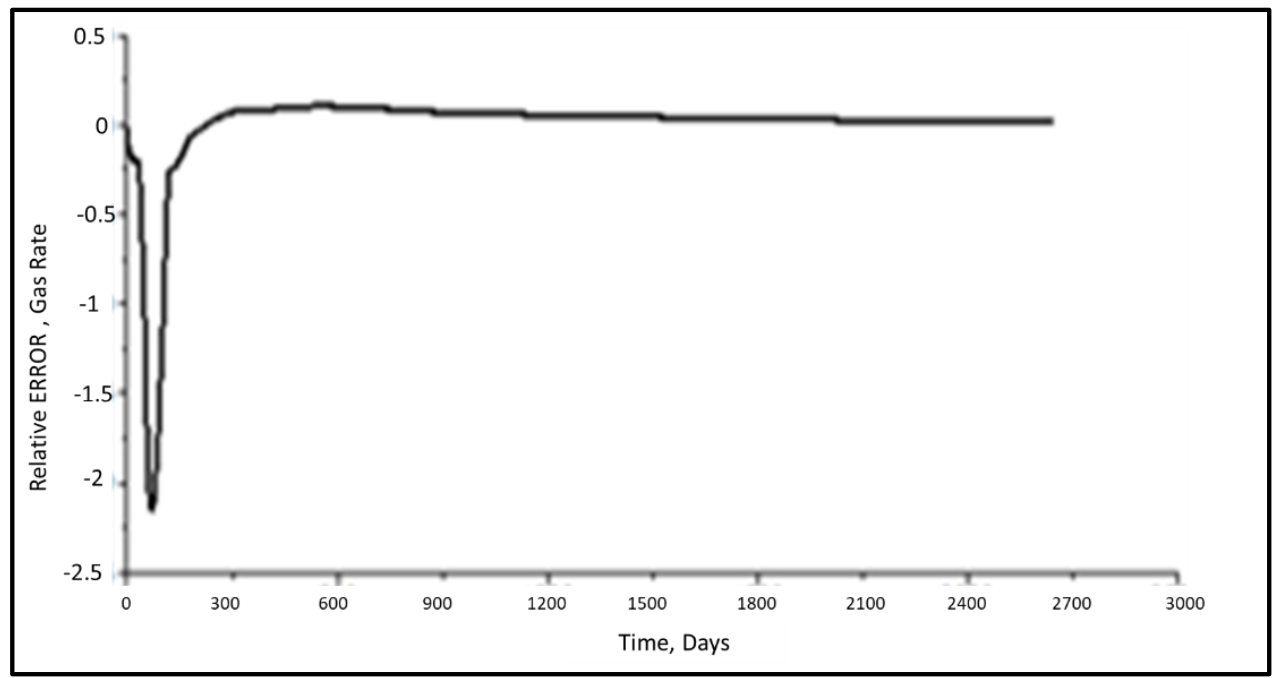

Figure 8-28: Relative Error in Gas Production between FD and SL Simulation cases (Miscible Gas).

Figure 8-29 shows the relative error of the gas injection and, as shown in the graph, initially this error is relatively high as it is reaches $10 \%$ for a short period, then flattens towards the end with almost no relative error.

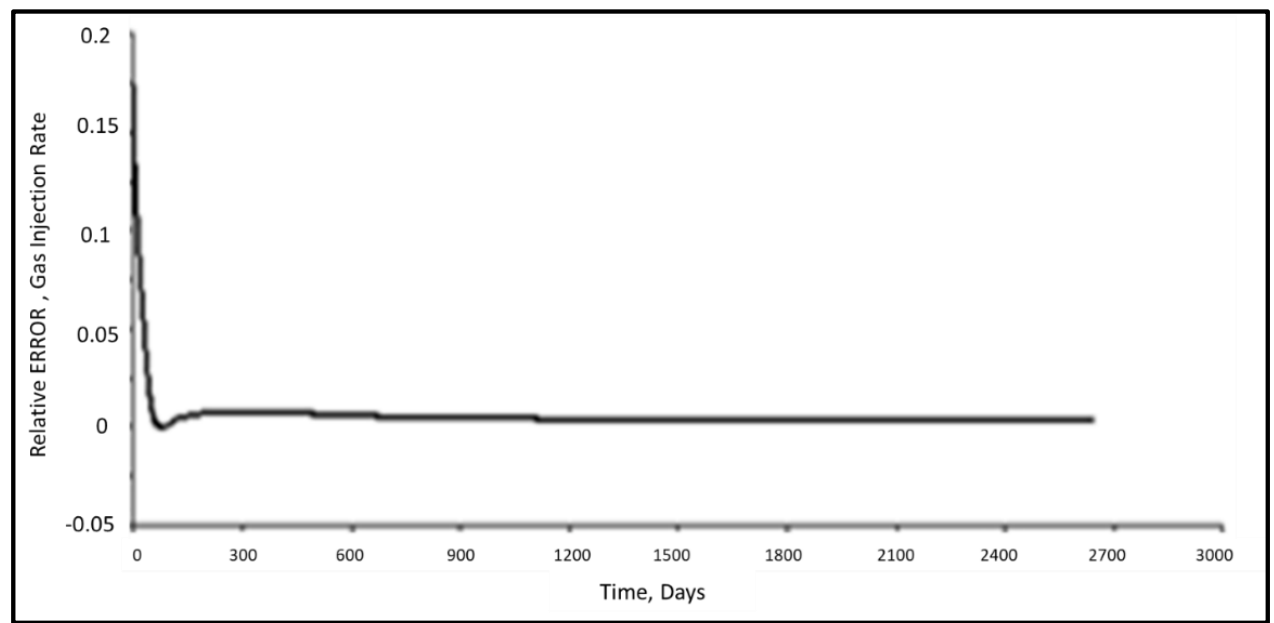

Figure 8-29: Relative Error in Gas Injection Rates between FD and SL Simulation cases (Miscible Gas). 


\subsubsection{FD VS SL 3D Analysis (Miscible Model)}

For the Miscible models (FD Vs SL Simulation), figure 8-30 below shows 3D oil saturation grid at the first-time step on the left and on the right shows at the end of simulation for both FD in the top and SL in the bottom for the miscible flood case.

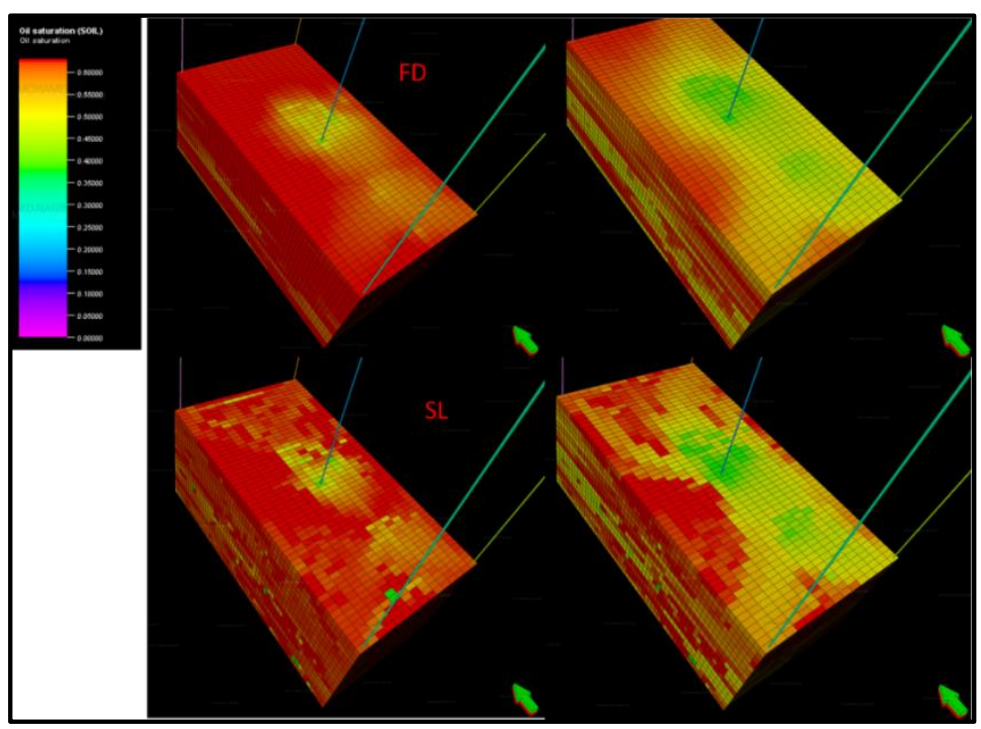

Figure 8-30: 3D Oil saturation Grid for Miscible Flood Model at $1^{\text {st }}$ and Last Timestep

As can be seen from the graph above, similar trends are observed in both models. Sliced 3D oil saturation grids for both FD and SL are presented in figures 8-31.

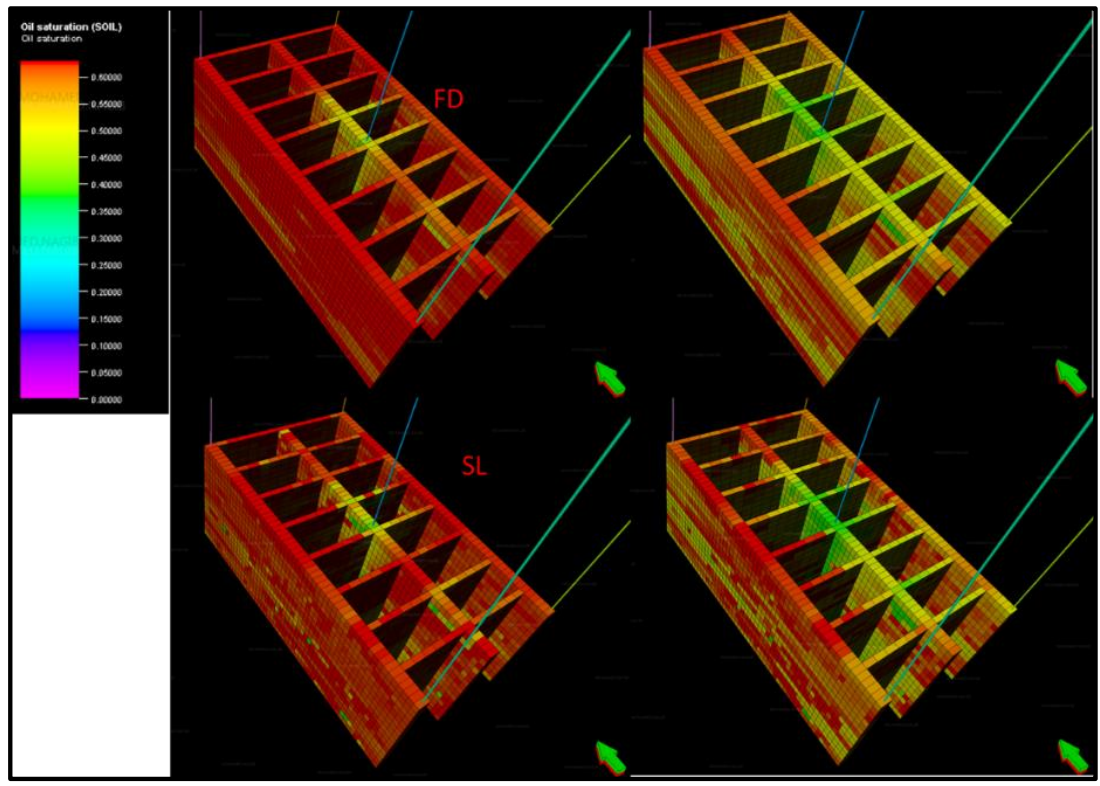

Figure 8-31: Sliced Oil saturation Grid for Miscible Flood Model at $1^{\text {st }}$ and Last Timestep 
Oil saturation grid cross section in I direction for both FD and SL presented in Figure 8-32 below;

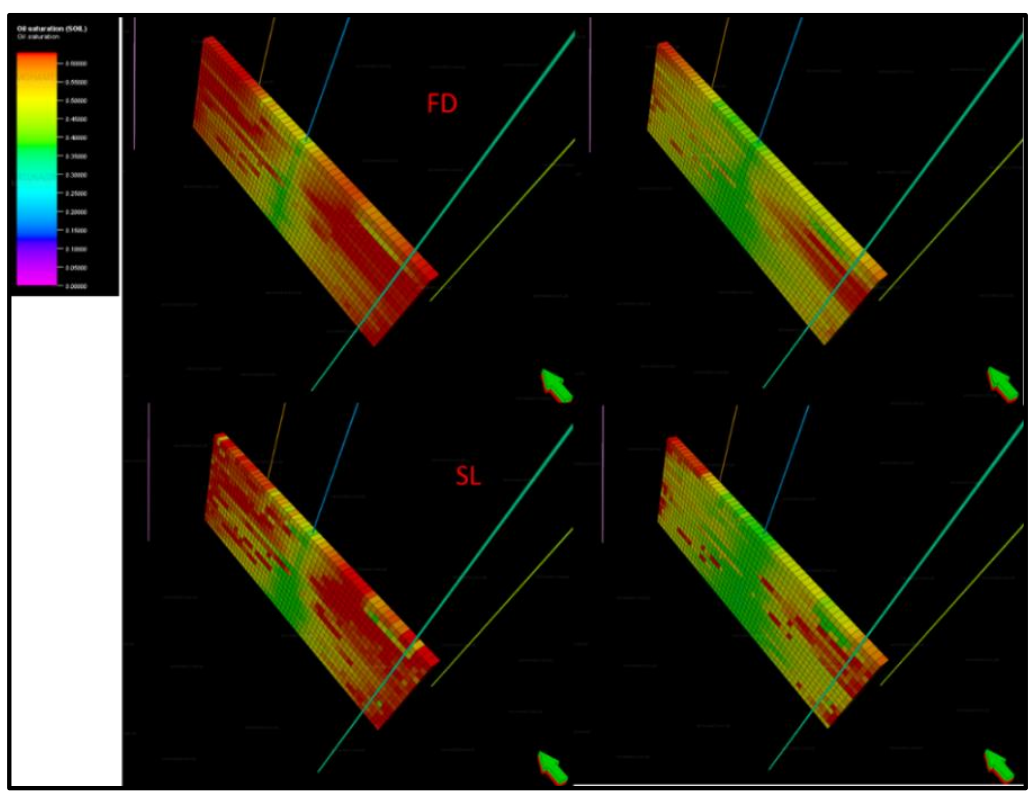

Figure 8-32: Oil saturation Grid cross section for Miscible Flood Model at $1^{\text {st }}$ and Last Timestep

No major differences were observed from different oil saturation grid section presented above. Similarly, 3D cross section of Gas saturation grid is presented in figures 8-33, 8-34 and 8-35.

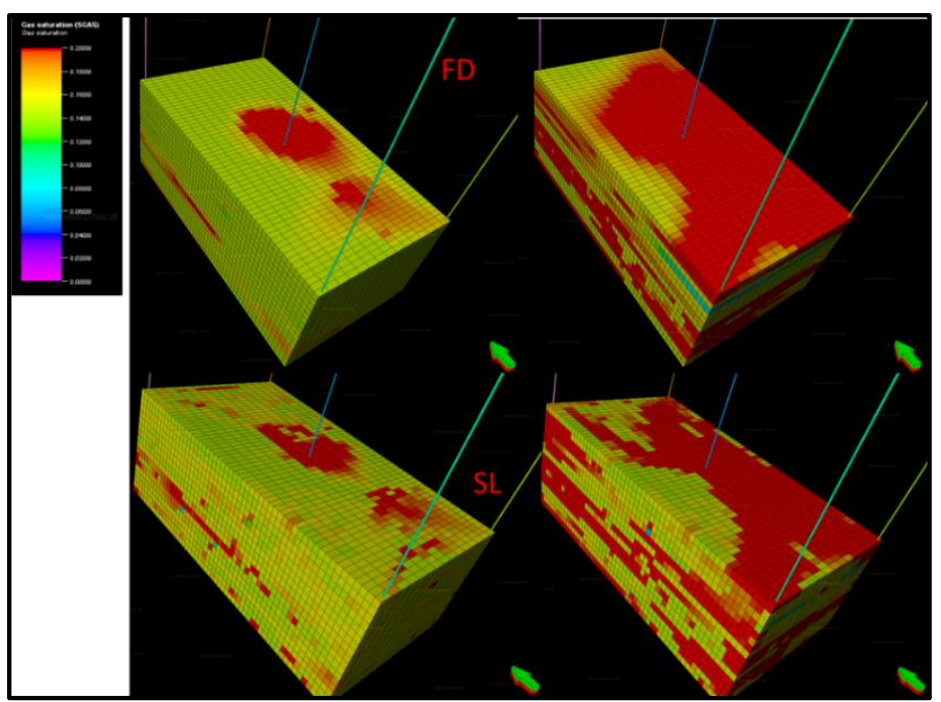

Figure 8-33: Gas Saturation Grid for Miscible Flood Model at 1st and Last Timestep 


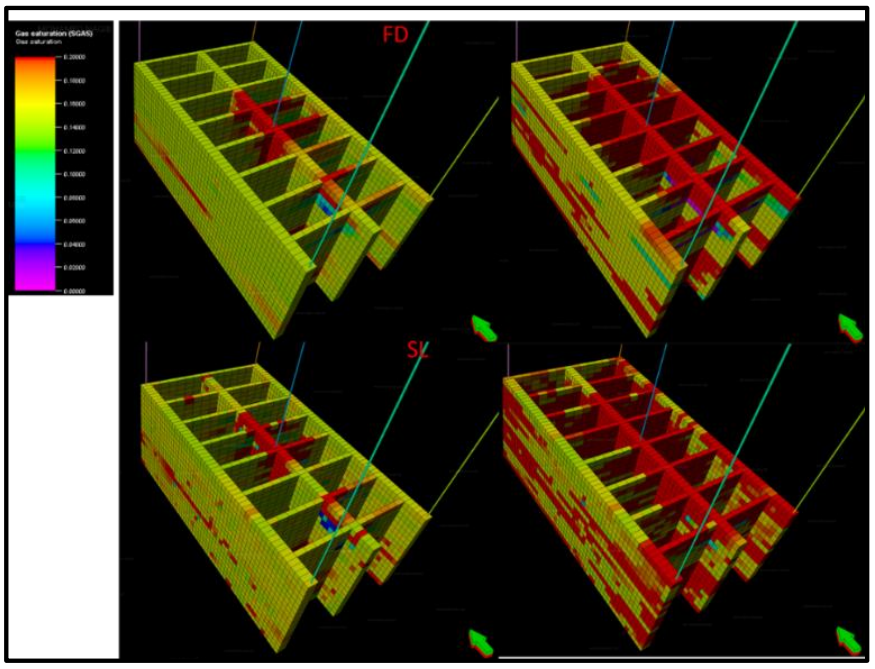

Figure 8-34: Sliced Gas Saturation Grid for Miscible Flood Model at $1^{\text {st }}$ and Last Timestep

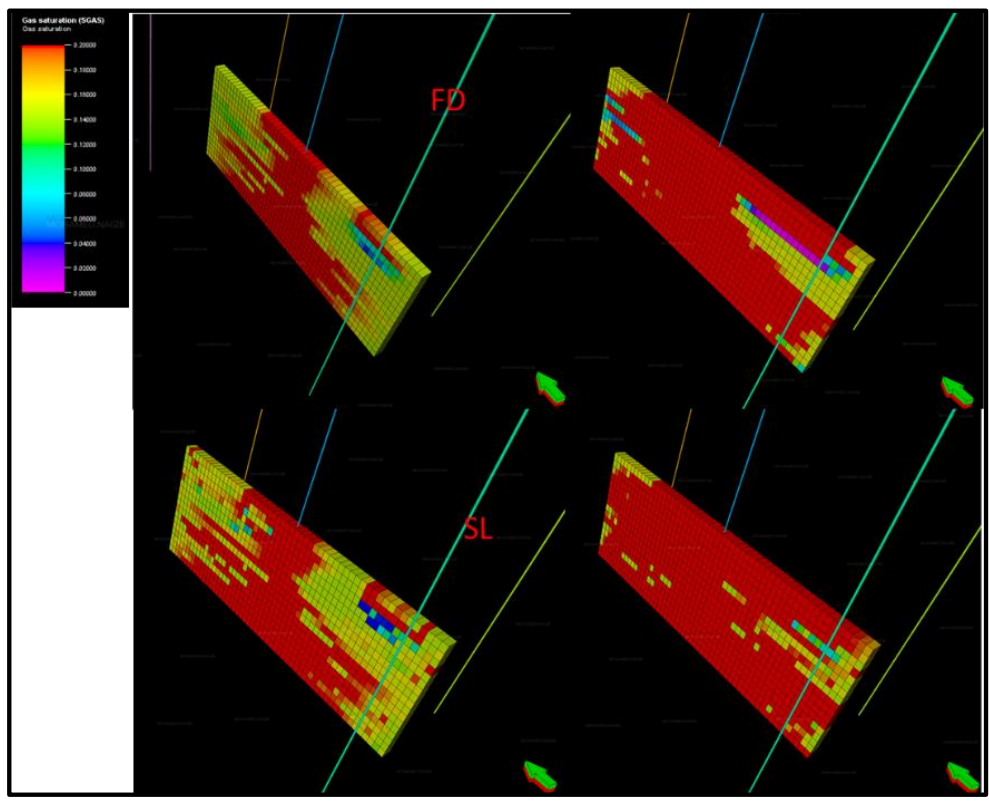

Figure 8-35: First- and Last-time steps of sliced 3D Gas saturation Grid (Miscible Flood FD Model)

As mentioned earlier in chapter 6, Oil Thickness Column (OTC) at the end of the field life and this property is calculated for both FD and SL grids according to its definition in Equation (6.2).

Figure 8-36 shows Compositional model OTC at the end of field life of both FD and SL models. 


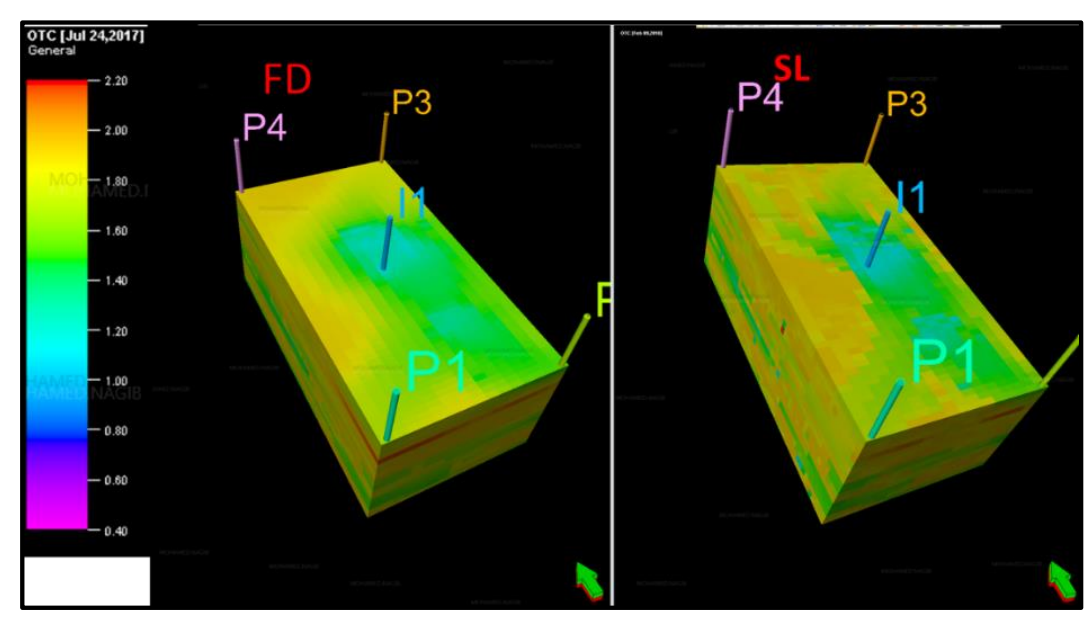

Figure 8-36: Miscible Flood Model OTC at the end of field life of both FD and SL models

\subsection{Conclusions:}

- The baseline gas injection case was modified to compositional mode for both SL and FD models by using different sets of PVT and relative permeability data.

- The same tuning parameters were used in the compositional mode, and the overall relative error noticed is low and within the acceptable limits.

- Comparisons of oil and water productions rates showed similar trends with minor differences between FD and SL compositional models

- Pressure trends are also similar in both models and the results showed an overall good match in all parameters.

- Oil and gas saturation grids comparisons for both FD and SL of compositional models.

- In terms of computational times comparisons, FD compositional model consumed twice times CPU consumed by SL compositional model.

- Low relative errors for all parameters for both FD and SL compositional models

- Similarly, the base case gas injection model is also modified to run in miscible flood modes for both SL and FD models by using different sets of PVT and relative permeability data.

- The results showed an overall good match in all parameters.

- Oil and gas saturation grids comparisons for both FD and SL of Miscible flood case.

- The same tuning parameters were used in the compositional model, and the overall relative error noticed is low and within the acceptable limits. 


\section{Chapter 9}

\section{Conclusions and Further Work}

\subsection{Summary of the Results}

\subsubsection{Relative Errors}

As a summary, Table 9-1 and Figure 9-1 represent the average error differences for the four different test simulation cases for oil rate, total oil production, gas production and gas injection rates. The highest error difference is in the case of high-resolution grid. As explained earlier, this is due to a material balance error caused when the number of grid cells was doubled keeping pore volume the same as the base case. Pore volume and oil in place were kept the same for fair comparison and assessment. The high resolution simulation case presented the highest error difference for both oil rate and total oil production. This should not be a generalised conclusion. The difference was not unexpected, and was mainly the result of a model limitation (keeping pore volume and STOIIP the same as the base case), where STOIIP is the Oil in place volumes in stock tank barrels units. SL simulation performance in high resolution grid is much closer to SL performance on coarse grid (Base Case). So, FD simulation has some conversion issues and performance was not stable.

Table 9-1: Summaries of the relative error between FD and SL in Simulation cases.

\begin{tabular}{|l|rr|r|r|r|}
\hline \multicolumn{1}{|c|}{ Case } & $\begin{array}{l}\text { Oil } \\
\text { Error diff } \%\end{array}$ & $\begin{array}{r}\text { Total } \\
\text { Error Diff } \%\end{array}$ & $\begin{array}{l}\text { Total Gas } \\
\text { Injection }\end{array}$ & $\begin{array}{l}\text { Total Gas } \\
\text { Production }\end{array}$ \\
\hline Base Case & $6 \%$ & $5 \%$ & $6 \%$ & $3 \%$ \\
\hline $\begin{array}{l}\text { High } \\
\text { Resolution }\end{array}$ & $7 \%$ & $9 \%$ & $6 \%$ & $5 \%$ \\
\hline Compositional & $2 \%$ & $2 \%$ & $5 \%$ & $1 \%$ \\
\hline \multicolumn{1}{|c|}{ Miscible } & $-3 \%$ & $-5 \%$ & $0 \%$ & $0 \%$ \\
\hline
\end{tabular}




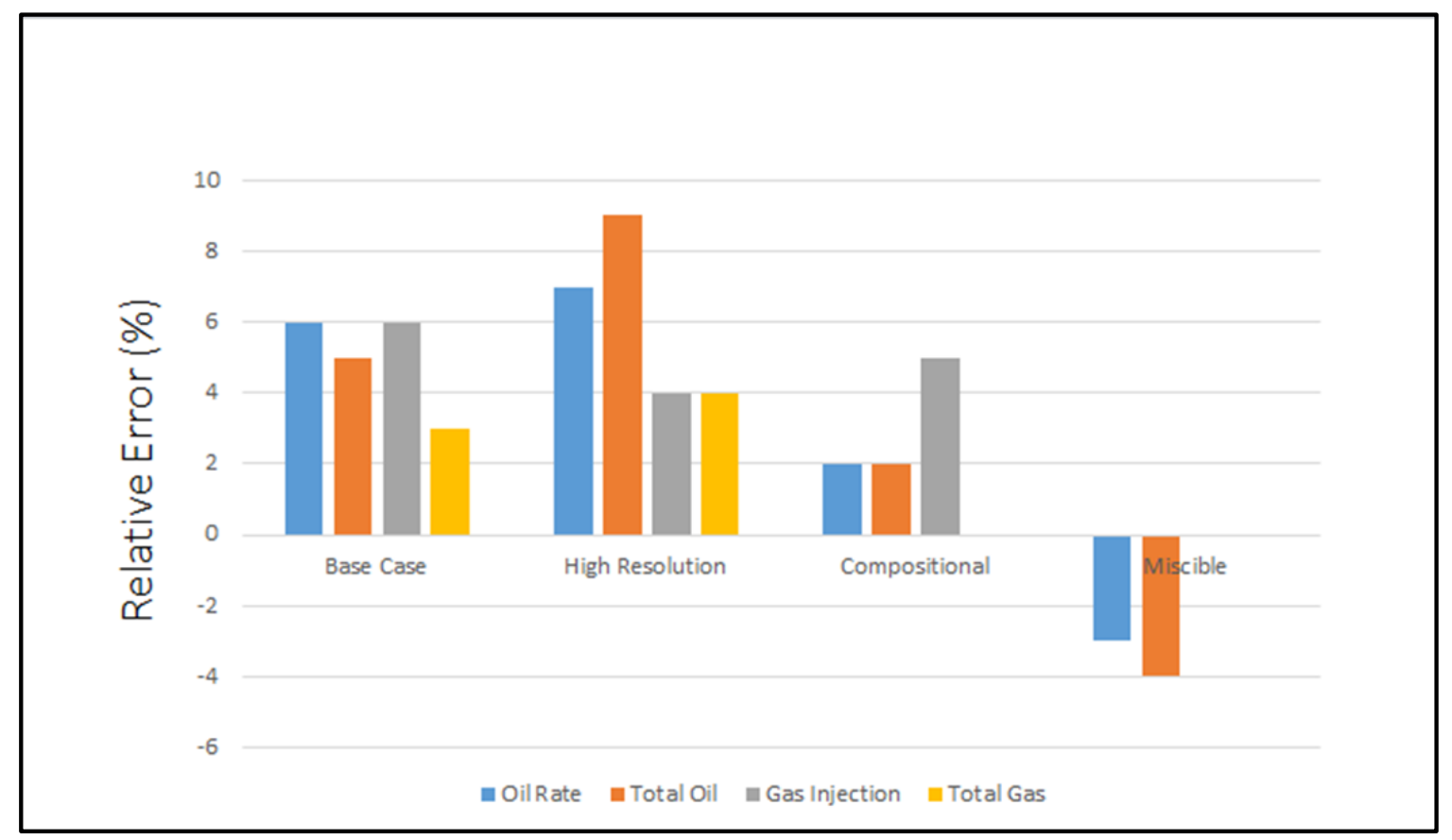

Figure 9-1: Relative Error \% between FD and SL Models

\subsubsection{Computational Performance Comparisons:}

To assess computational calculation times and simulator performance, Figure 9-2 presents the total CPU consumed in the cases of FD vs. SL in the four tested cases. As can be seen from the summary plots below, FD simulation consumed almost double of the CPU consumed by SL. 


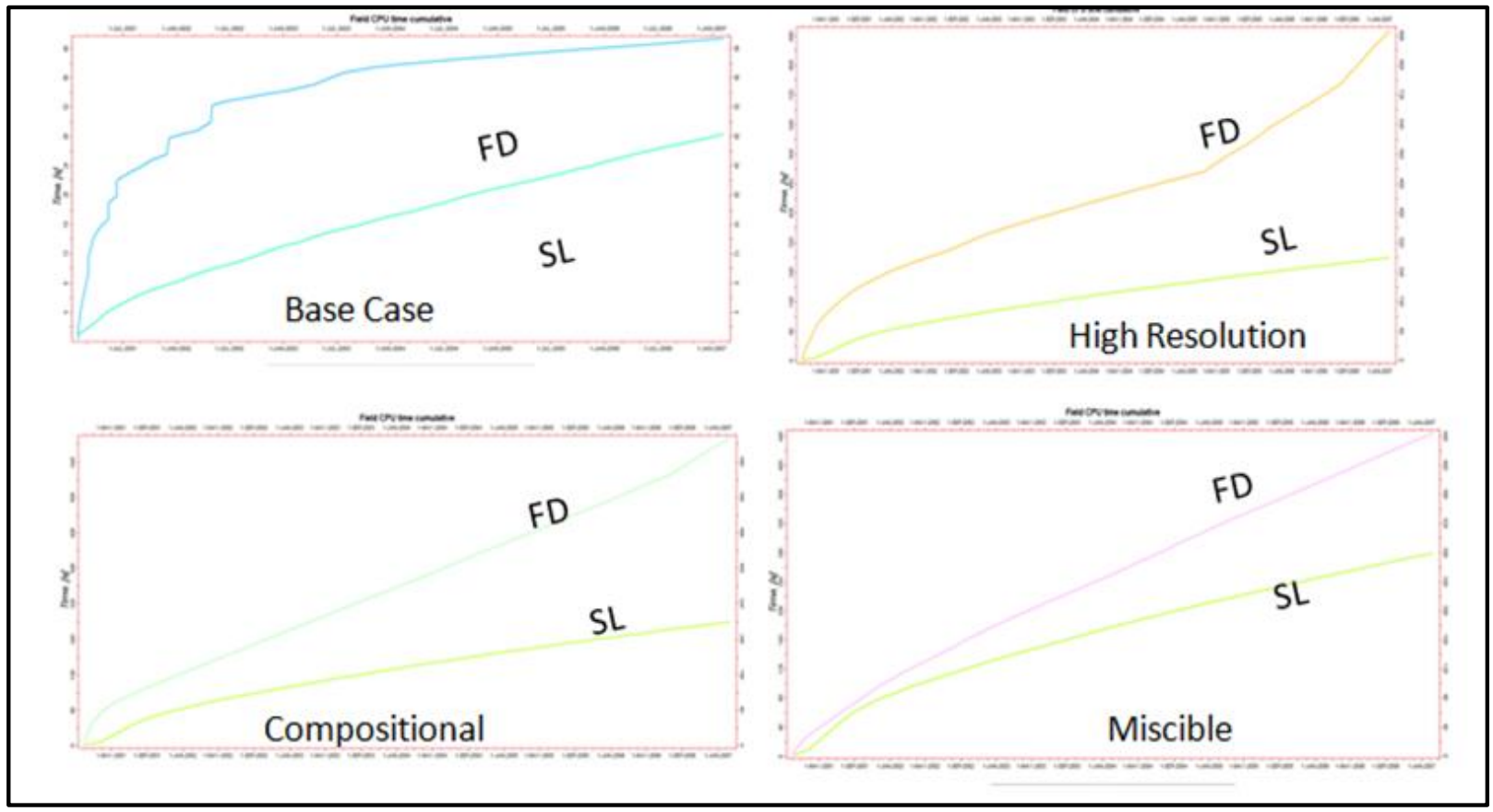

Figure 9-2: Total CPU vs. Time for Gas injection models in FD and SL.

Table 9-2 and Figure 9-3 summarise and compare total CPU consumed in both FD and SL simulation models in all four tested cases.

Table 9-2: Summaries Total CPU used by FD and SL

\begin{tabular}{|l|c|c|}
\hline \multicolumn{1}{|c|}{ Case } & TCPU, seconds - SL & TCPU, seconds - FD \\
\hline Base Case & 28 & 42 \\
\hline High Resolution & 480 & 880 \\
\hline Compositional & 280 & 440 \\
\hline Miscible & 280 & 650 \\
\hline
\end{tabular}




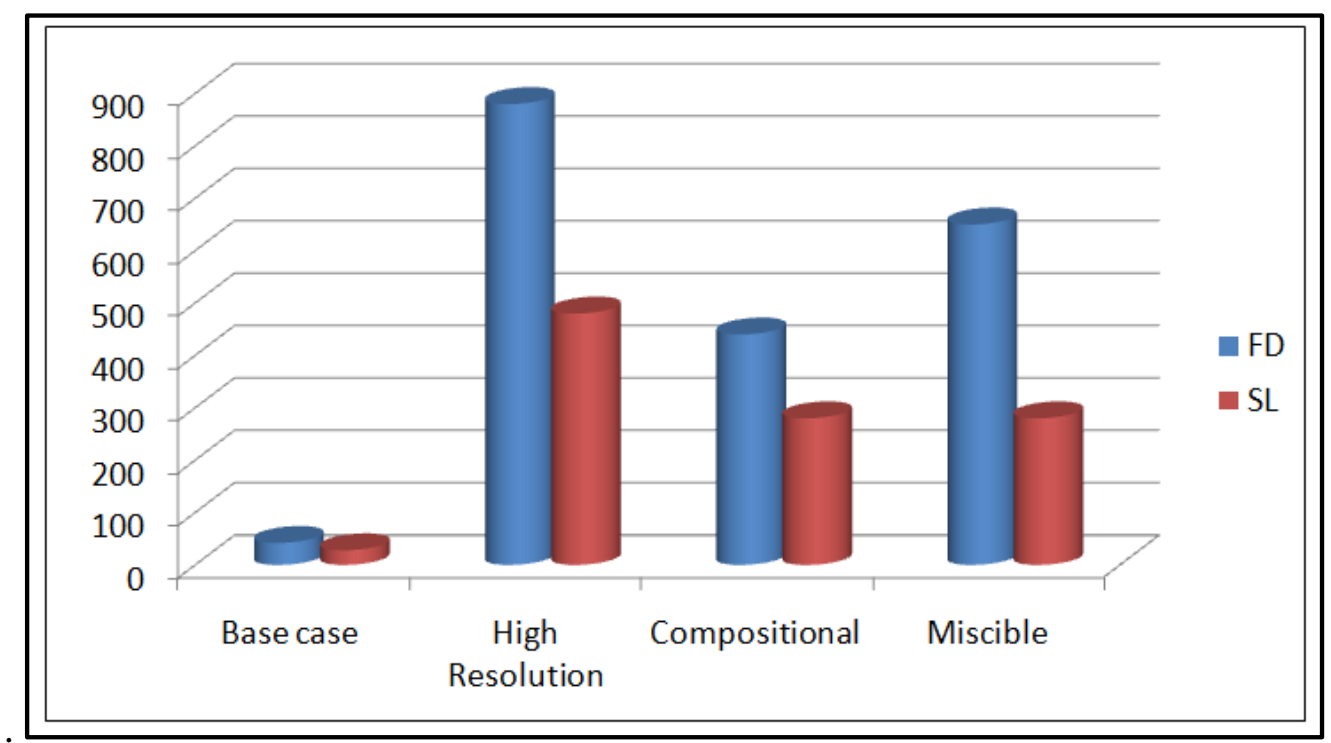

Figure 9-3: Comparison of Total CPU for Gas injection models in FD and SL.

\subsubsection{D Oil Saturation Grid Comparisons}

Comparison of 3D oil saturation grid of Black oil base case for both FD and SL are presented in figure 9-4. As can be noticed and observed earlier, very minor change in oil saturation noticed in base case gas injection FD and SL model. In the top, oil saturation grid at initialisation, after 180 days and end of simulation FD model case and in the bottom are SL case at the same time steps.

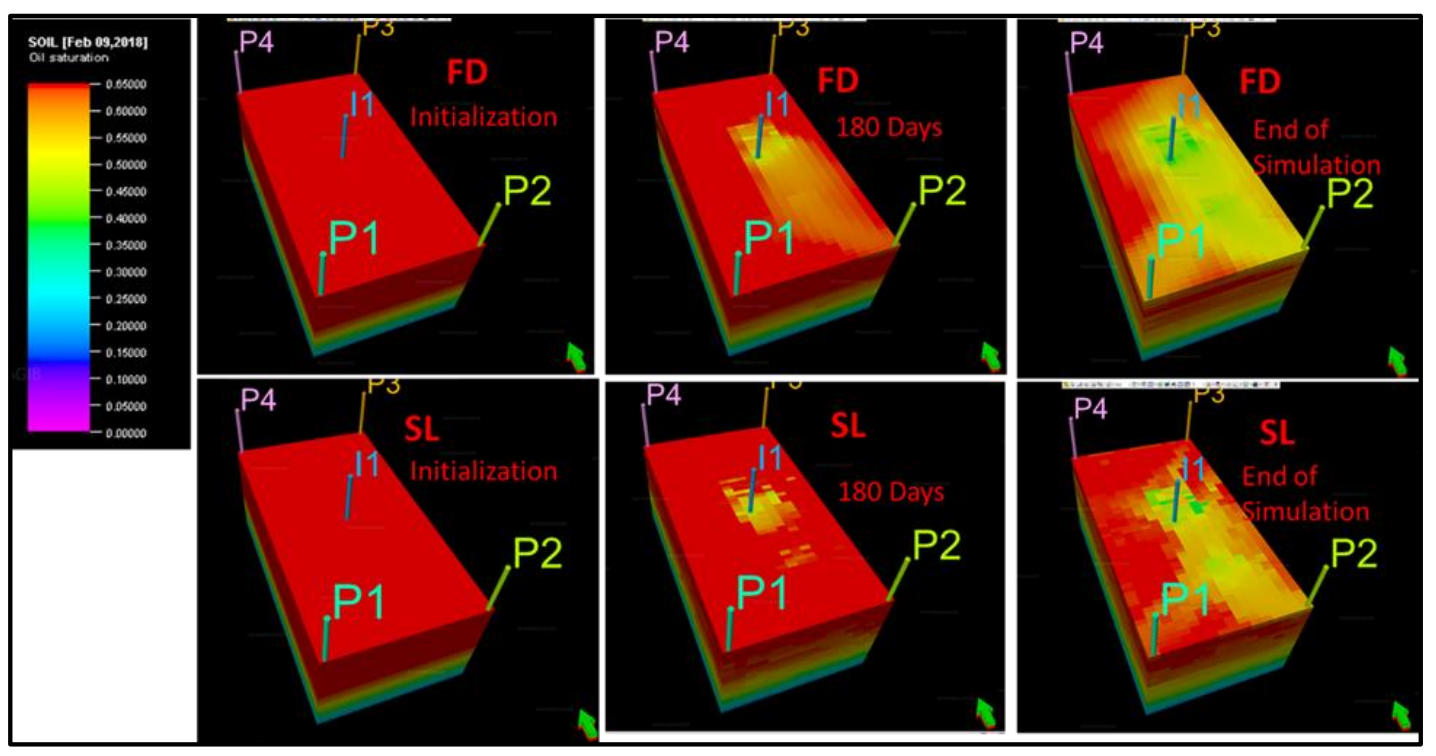


Figure 9-4: 3D Oil Saturation Grid Comparisons of Black Oil Base for both FD and SL cases.

Similarly, high resolution gas injection case is presented below in figure 9-5. As can be seen, minor changes are noticed at the first-time step and some changes are noticed at the end of simulation.

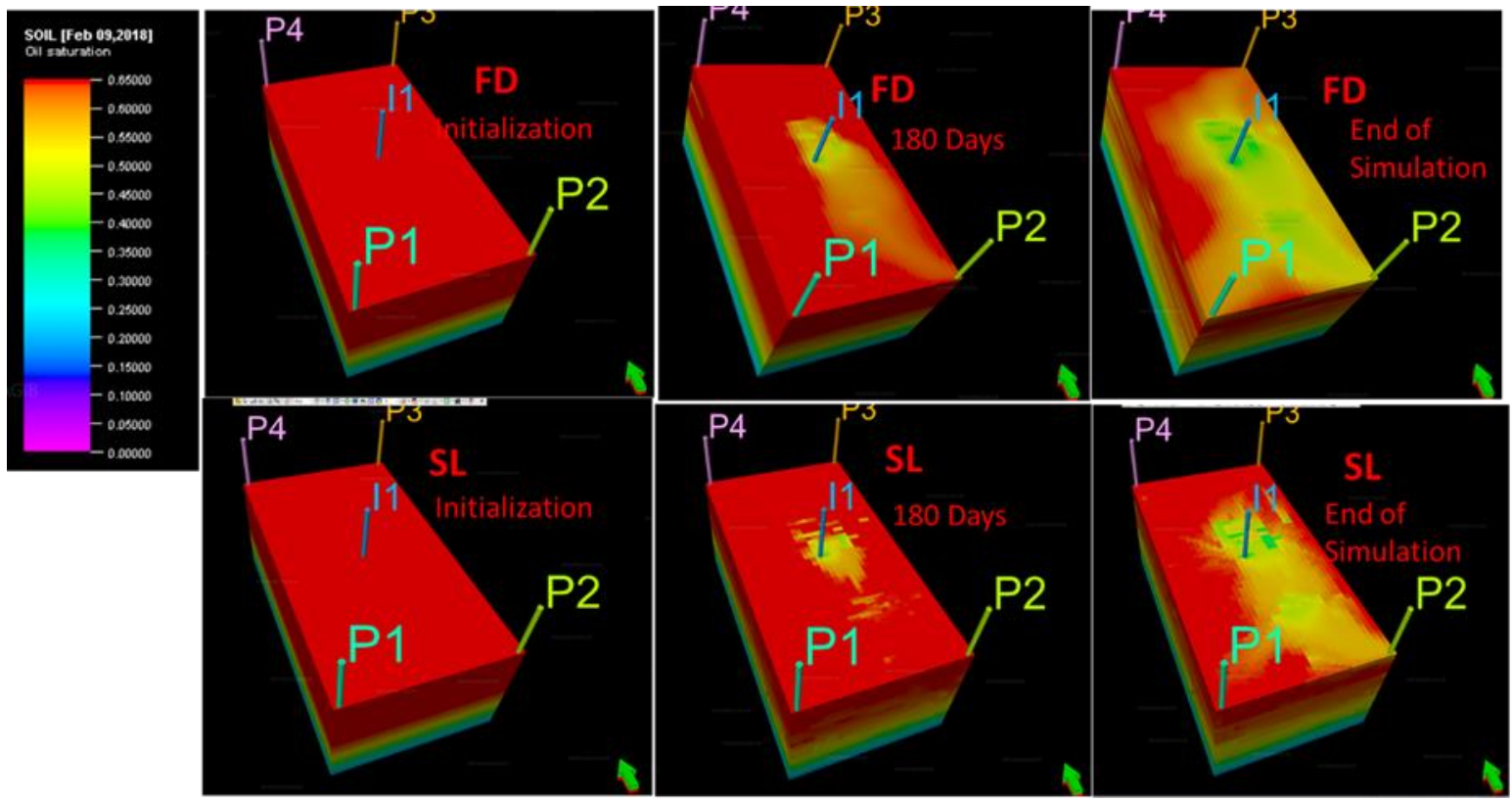

Figure 9-5: 3D Oil Saturation Grid Comparisons of High-Resolution case for both FD and SL cases.

Oil saturation grid comparison for compositional are presented in Figure 9-6. As can be seen, to the top is FD compositional model. However, to the bottom is SL case. General trends are noticed in both models with minor differences. 


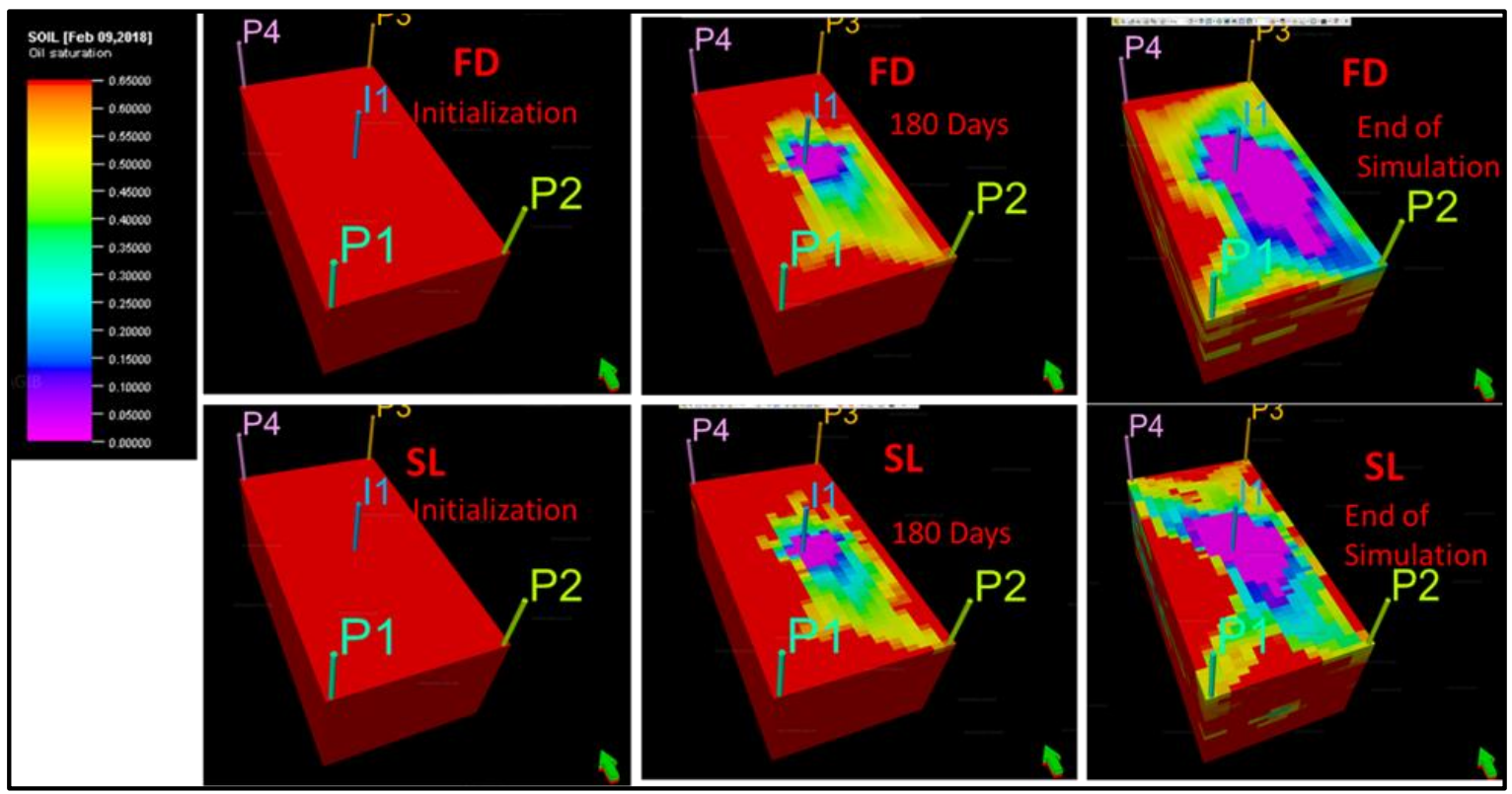

Figure 9-6: 3D Oil Saturation Grid Comparisons of the Compositional case for both FD and SL cases.

Oil saturation grid comparison for Miscible are presented in Figure 9-7. As can be seen, to the top is FD Miscible flood model. However, to the bottom is SL case. General trends are noticed in both models with minor differences.

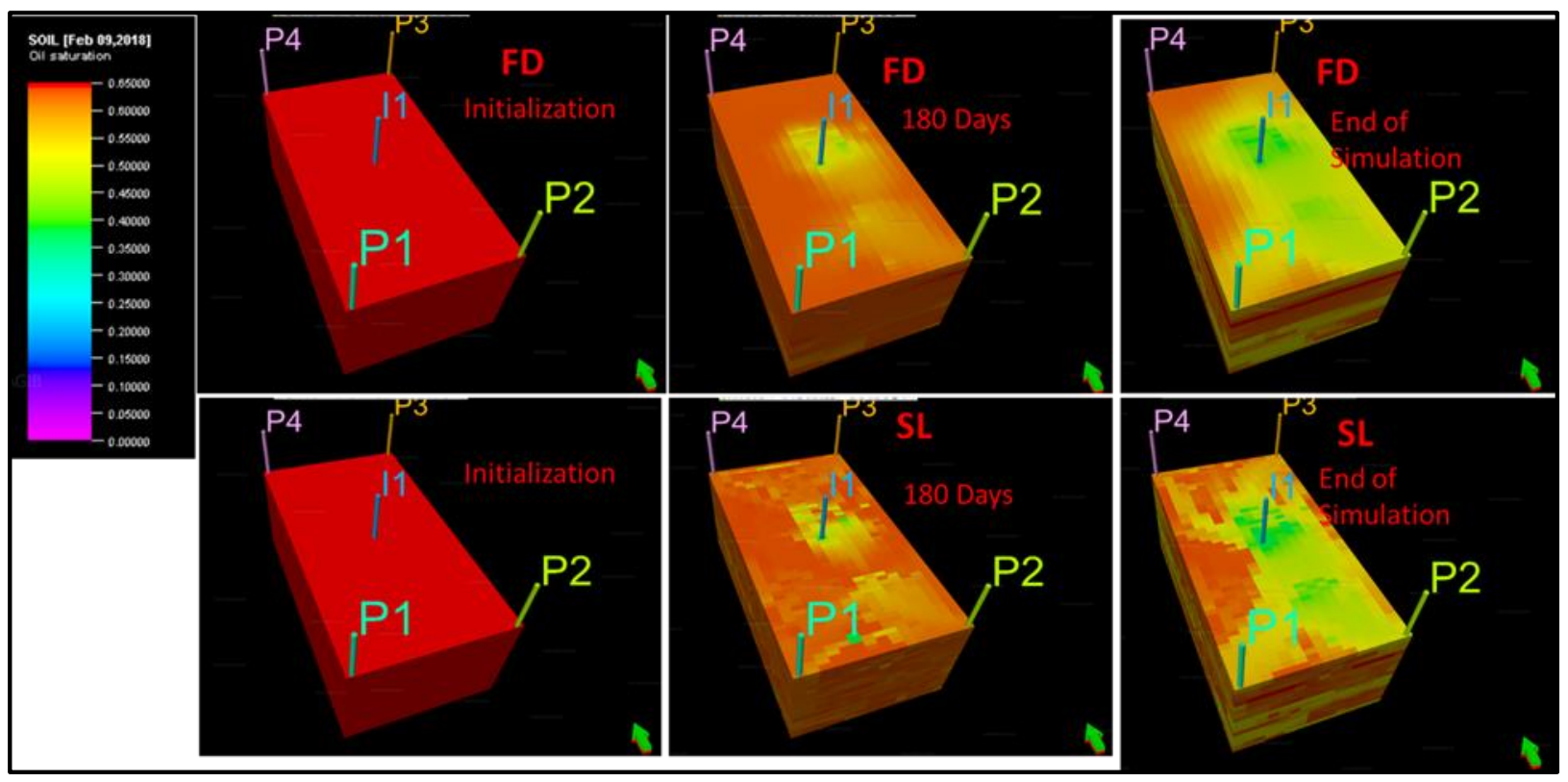


Figure 9-7: 3D Oil Saturation Grid Comparisons of Miscible flood model for both FD and SL cases.

\subsubsection{D Gas Saturation Grid Comparisons}

Similar to oil saturation summary grids presented in the previous section. Gas saturation grid comparison summary presented in figure 9-8. As can be noticed from the graph below, FD saturation at the top and 3 grids presented: at initialisation, after 180 days and at the end of simulation. Similarly, SL gas saturation summary at the bottom. Some minor change in gas saturation noticed in base case gas injection FD and SL model.

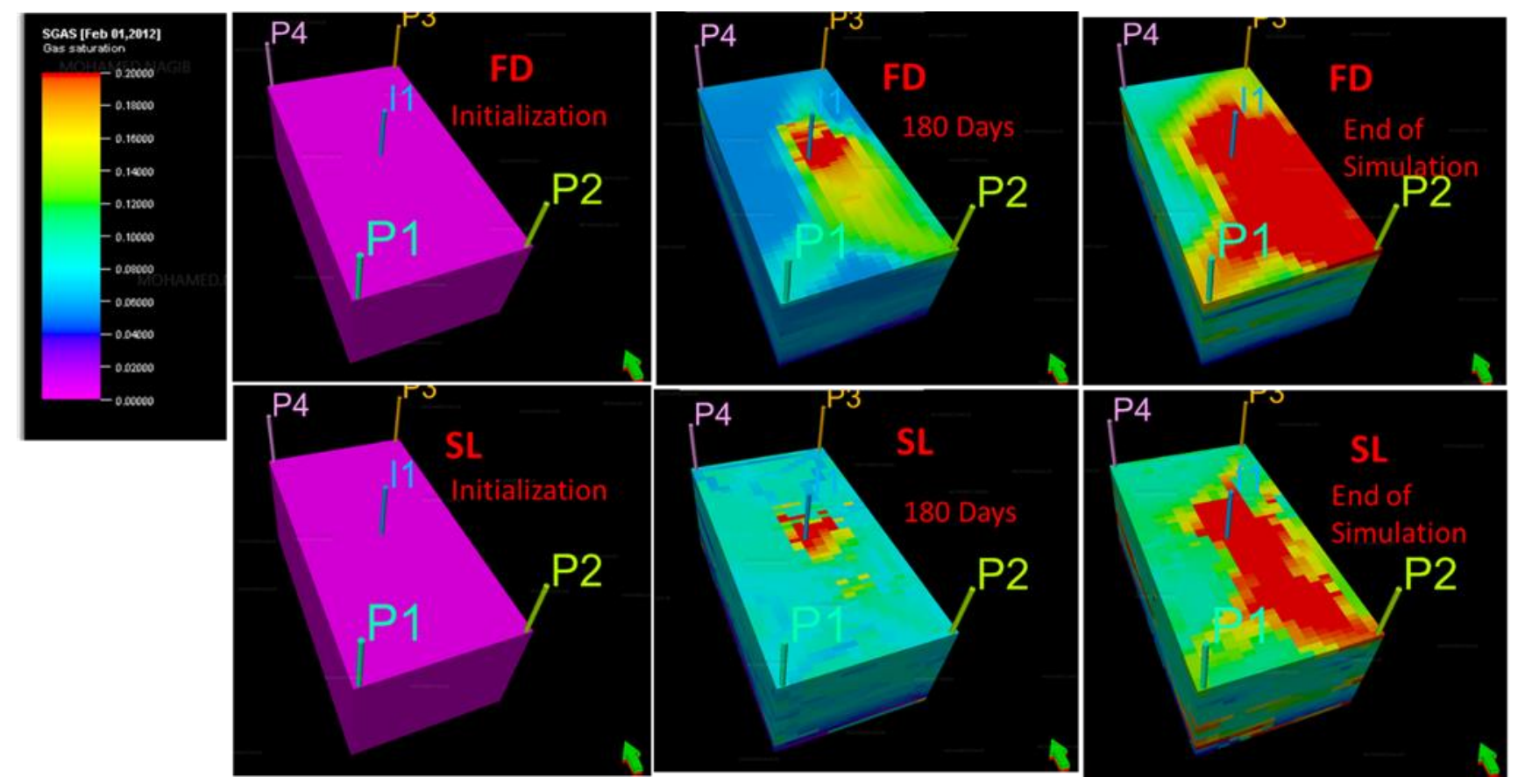

Figure 9-8: 3D Gas Saturation Grid Comparisons of Black Oil Base for both FD and SL cases.

With regards to high resolution grid, figure 9-9 presents 3D gas saturation summary. FD 3D gas oil saturation at the top and SL at the bottom. 


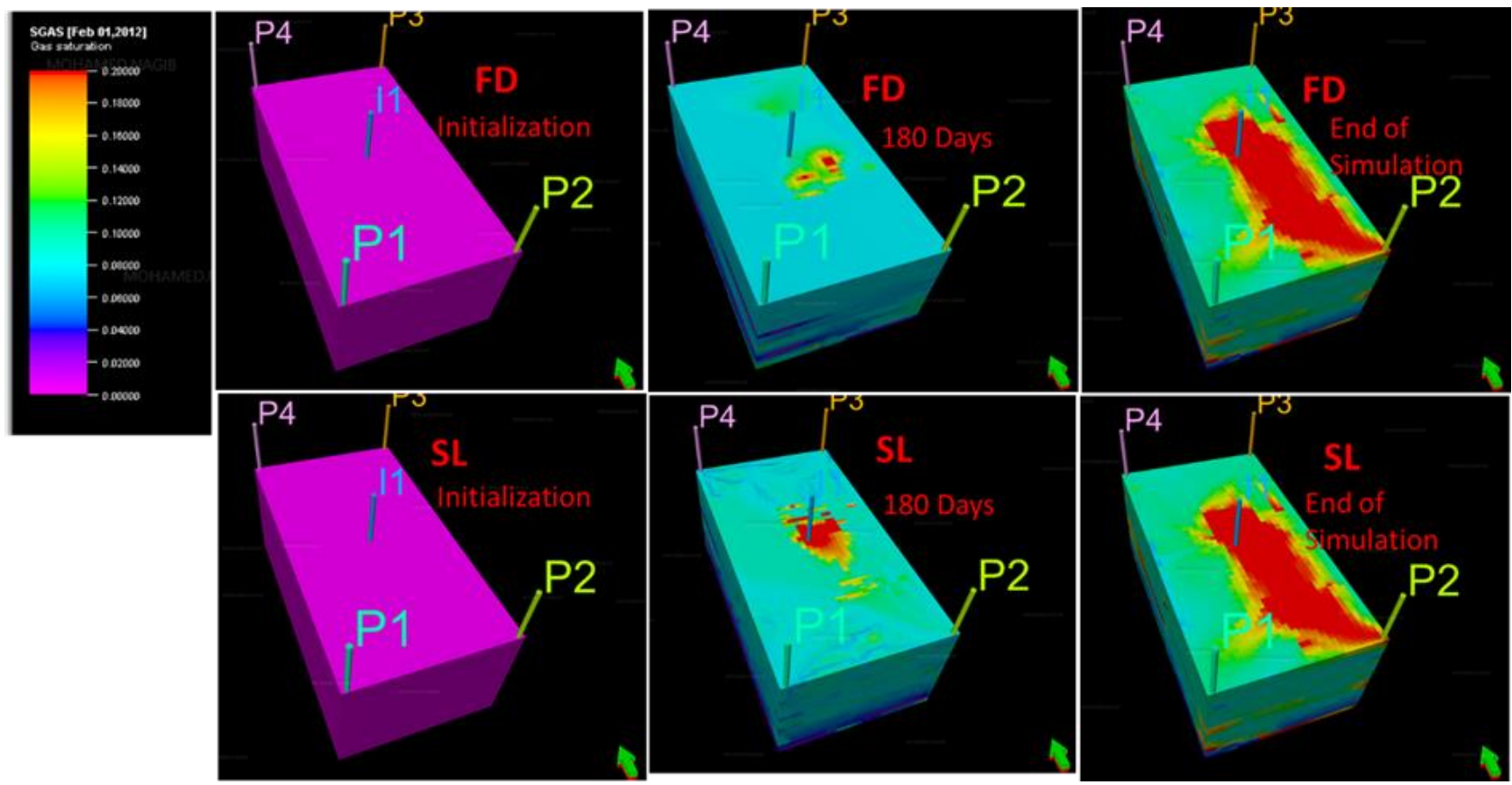

Figure 9-9: 3D Gas Saturation Grid Comparisons of High-Resolution model for both FD and SL cases.

3D Gas saturation grid summary of compositional is presented in figure 9-10.

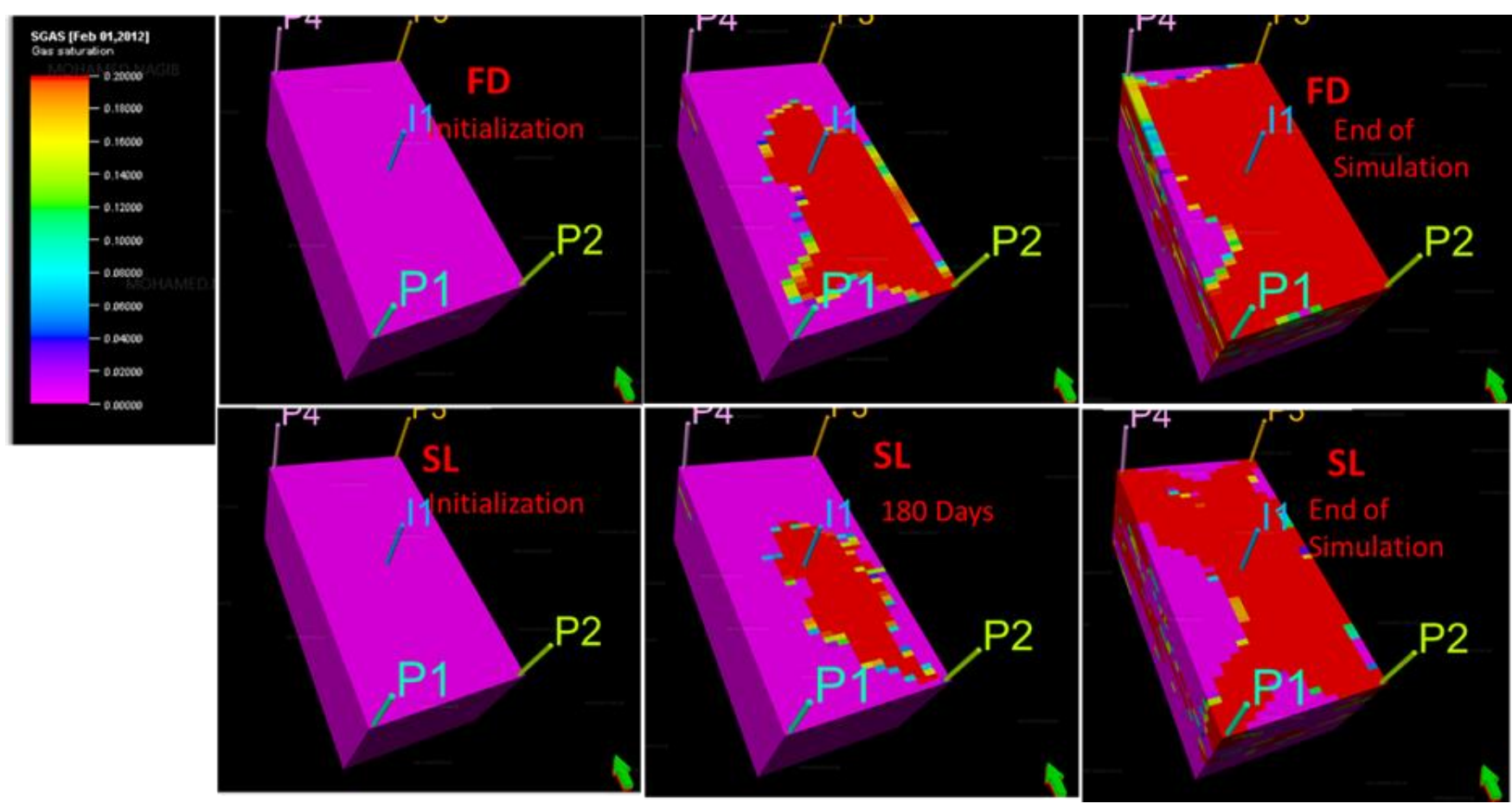


Figure 9-10: 3D Gas Saturation Grid Comparisons of Compositional model for both FD and SL cases.

Additionally, 3D Gas saturation grid summary of Miscible flood is presented in figure 9-11.

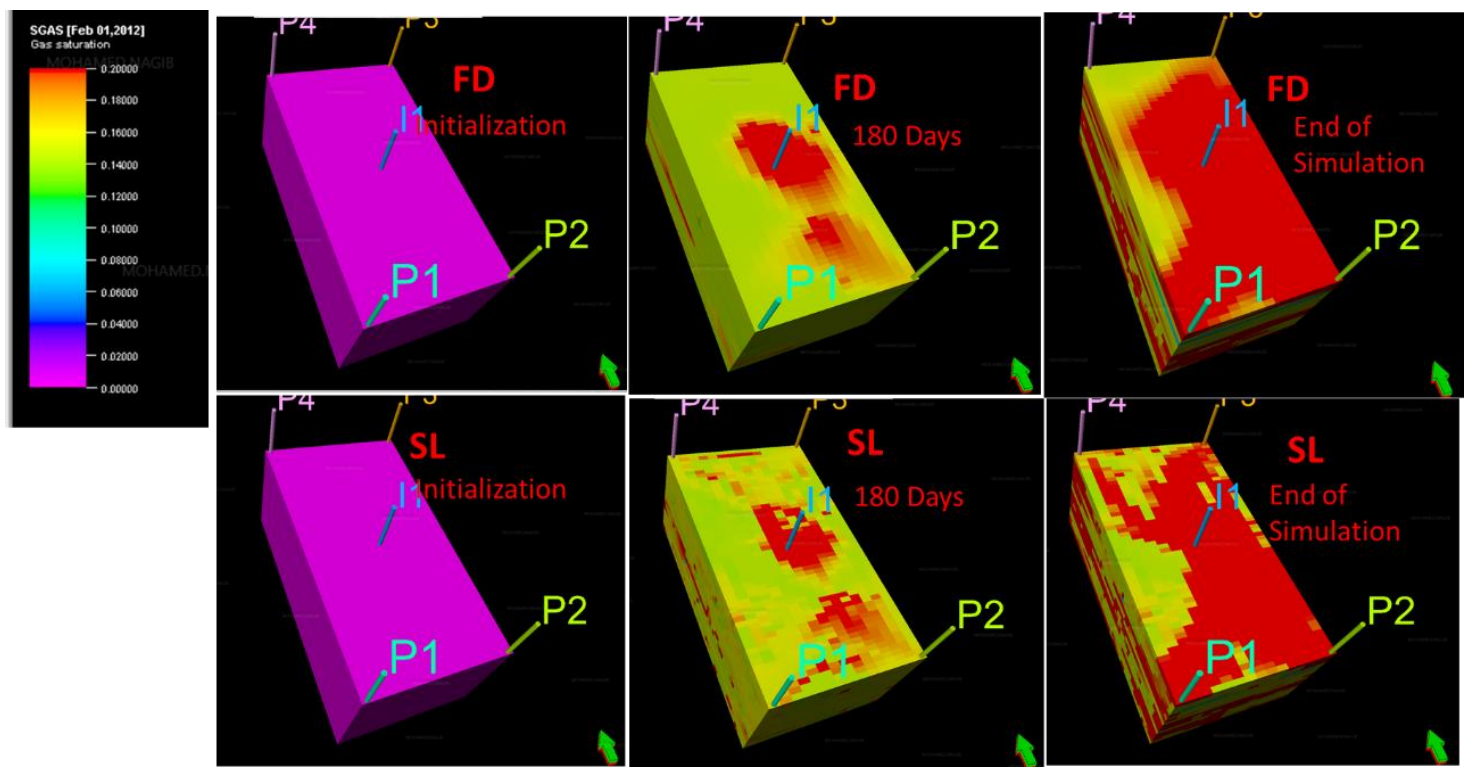

Figure 9-11: 3D Gas Saturation Grid Comparisons of Miscible Flood model for both FD and SL cases.

\subsubsection{Oil Thickness Column Analysis (OTC):}

The OTC grid results were presented earlier for each model as the OTC was calculated for base case gas injection model case and presented in chapter 6, High resolution grid presented in chapter 7, compositional and Miscible flood models in chapter 8.

After calculating OTC for each model. Then, a new property was generated to calculated the difference of OTC at the end of simulation for each model in both FD and SL cases. The property calculated as per the following equation in each grid cell:

OTC Difference $=$ OTC $($ FD) - OTC $($ SL)

As mentioned earlier the bases OTC calculations was Oil saturation times of Cell thickness.

Figure 9-12 presents OTC differences of the base case gas injection model. 


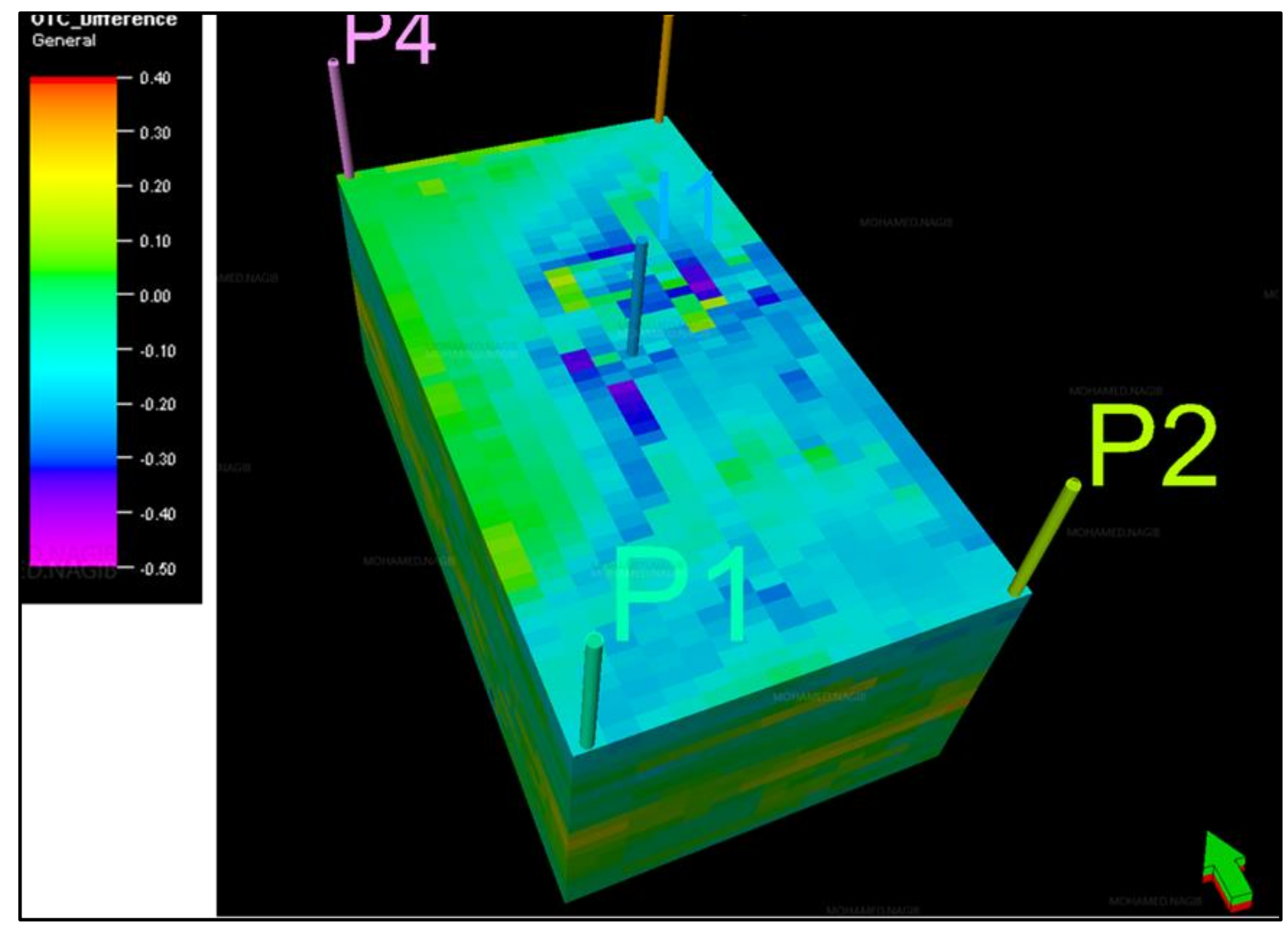

Figure 9-12: OTC Differences of the base Case Gas Injection Model.

As shown on the figure above and based on the scale on the left-hand side, differences occur in a range between 0.5 to -0.5 difference in oil saturation between FD and SL. Positive difference means that the FD value is higher than SL, while negative values indicate SL is higher compare to FD. From the 3D graphs most of the areas shows green colour in which indicated that the difference is minor, apart from the area around the injector in the middle and oil producer P2.

The High-Resolution case OTC difference is presented in Figure 9-13 


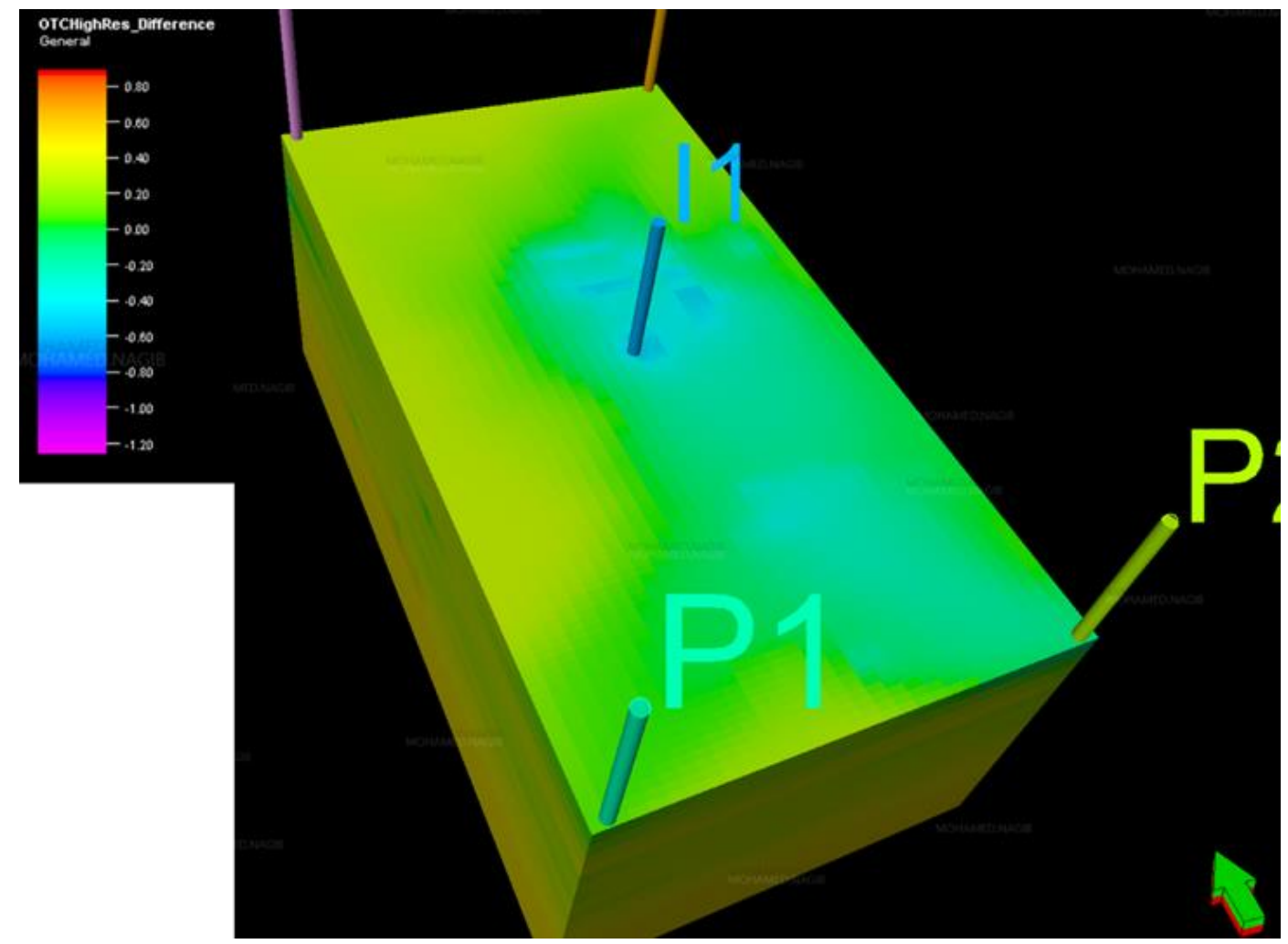

Figure 9-13: OTC Differences of High-Resolution Gas Injection Model.

As shown on the figure above and similar to the same explanation earlier for the base case gas injection, differences occur in a range between 0.5 to -0.5 difference in oil saturation between FD and SL. Positive difference means that FD value is higher than SL, while negative values indicate SL is higher compare to FD. From the 3D graphs most of the areas shows green colour in which indicated that the difference is minor.

Figure 9-14 shows OTC differences of the compositional model case. 


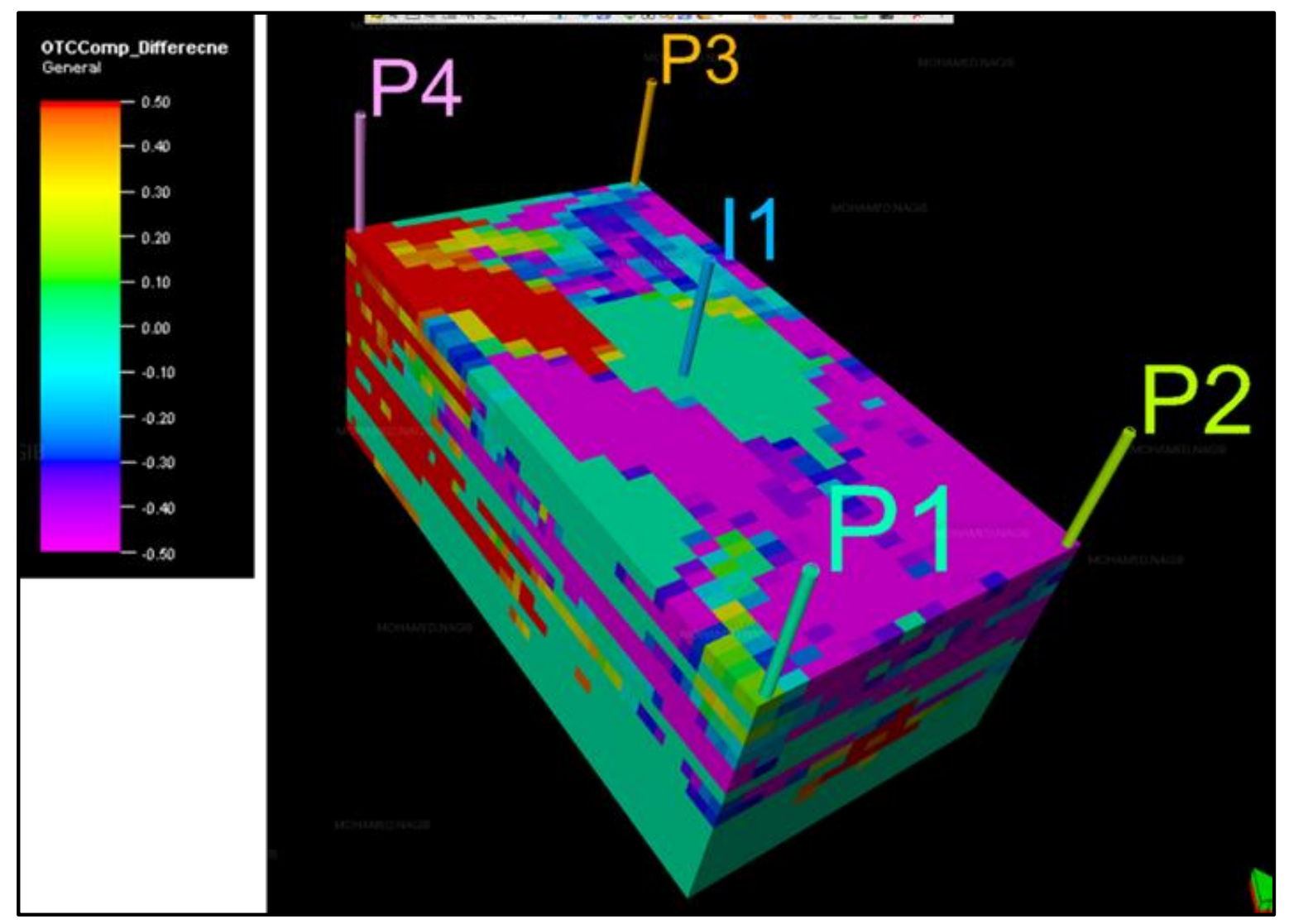

Figure 9-14: OTC Differences of Compositional Gas Injection Model.

As shown in the figure above some areas show negative values in purple colours and this is indicates that SL oil saturation values is higher than the values calculated by FD. As in the compositional model FD case was made via EOS which is totally different than black oil simulation. So, SL compositional model behaves a little differently in comparison to FD.

And for the last case model, Figure 9-15 shows OTC differences of Miscible flood model. 


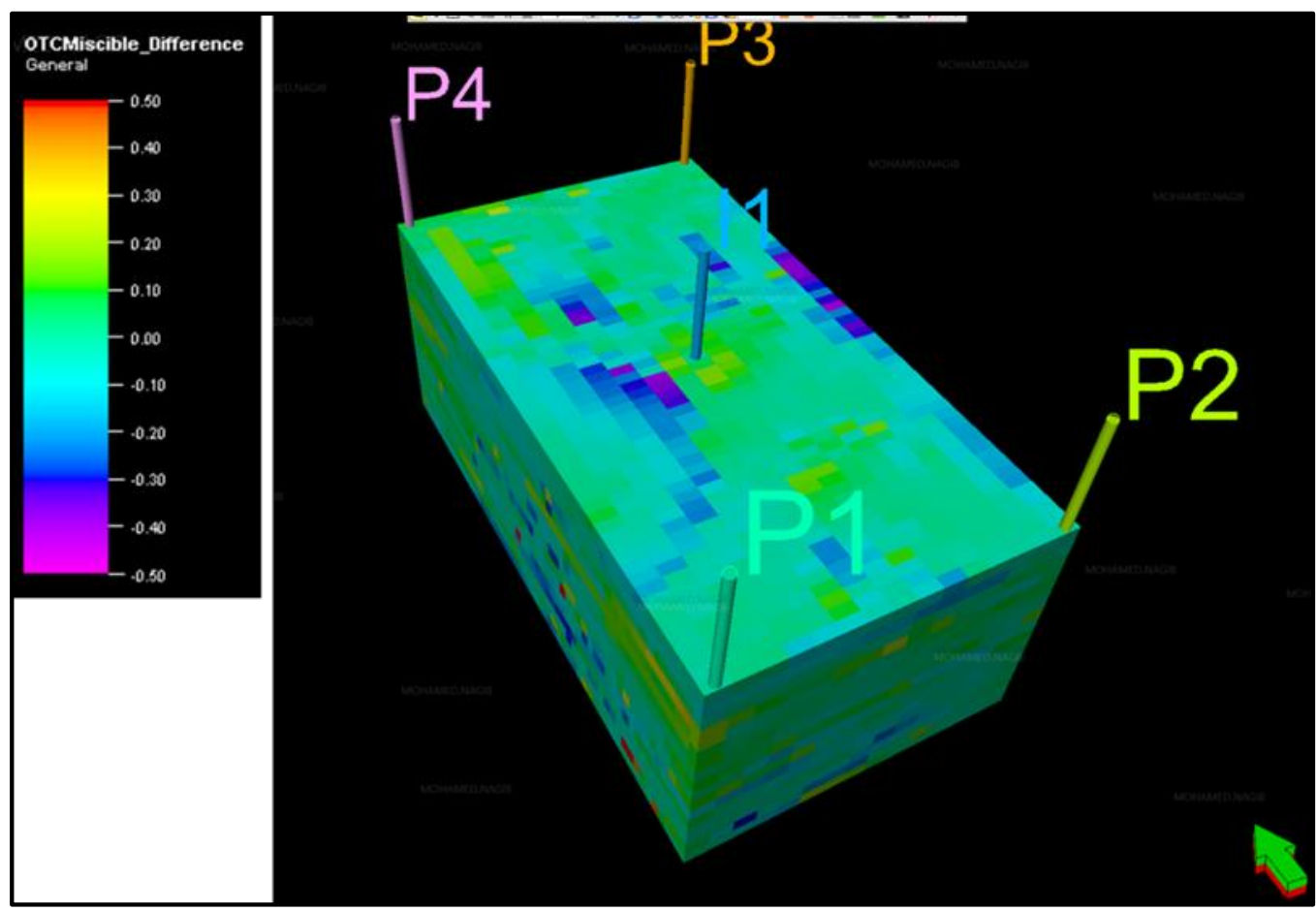

Figure 9-15: OTC Differences of Miscible Flood Gas Injection Model.

As shown in the above figure minor differences are noticed in the middle of the grid only to the left side of the injector. However, the majority of the grid shows very minimum difference as per the green colour.

\subsubsection{Wells Production Data Analysis within 3D Grid:}

For the well production comparison, for each model tested, bubble maps were created on 3D and production for each of the producers was presented for both FD and SL model per each tested case.

Figure 9-16 shows the base case gas injection model. As per the graph below, to the left is shown the total oil production per each producer of the FD model, and to the right is shown the same for SL model, both at the end of simulation. 


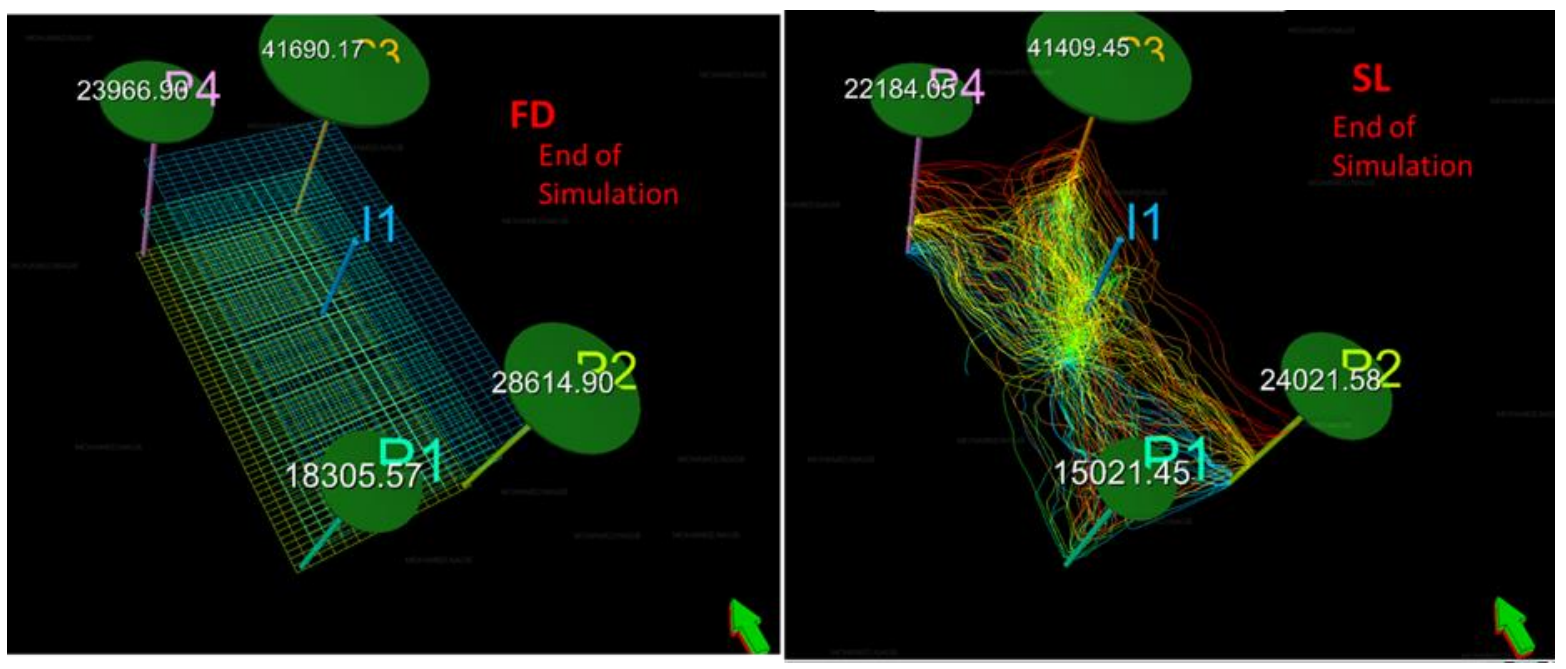

Figure 9-16: Total oil production per each well with 3D grid of the base case model.

As can be seen from the graph above, production data per well for both FD and SL models are comparable.

Figure 9-17 shows the results for the high-resolution gas injection model. As per the graph below, to the left is shown the total oil production per each producer of the FD model, and to the right is shown the same for SL model, both at the end of simulation.
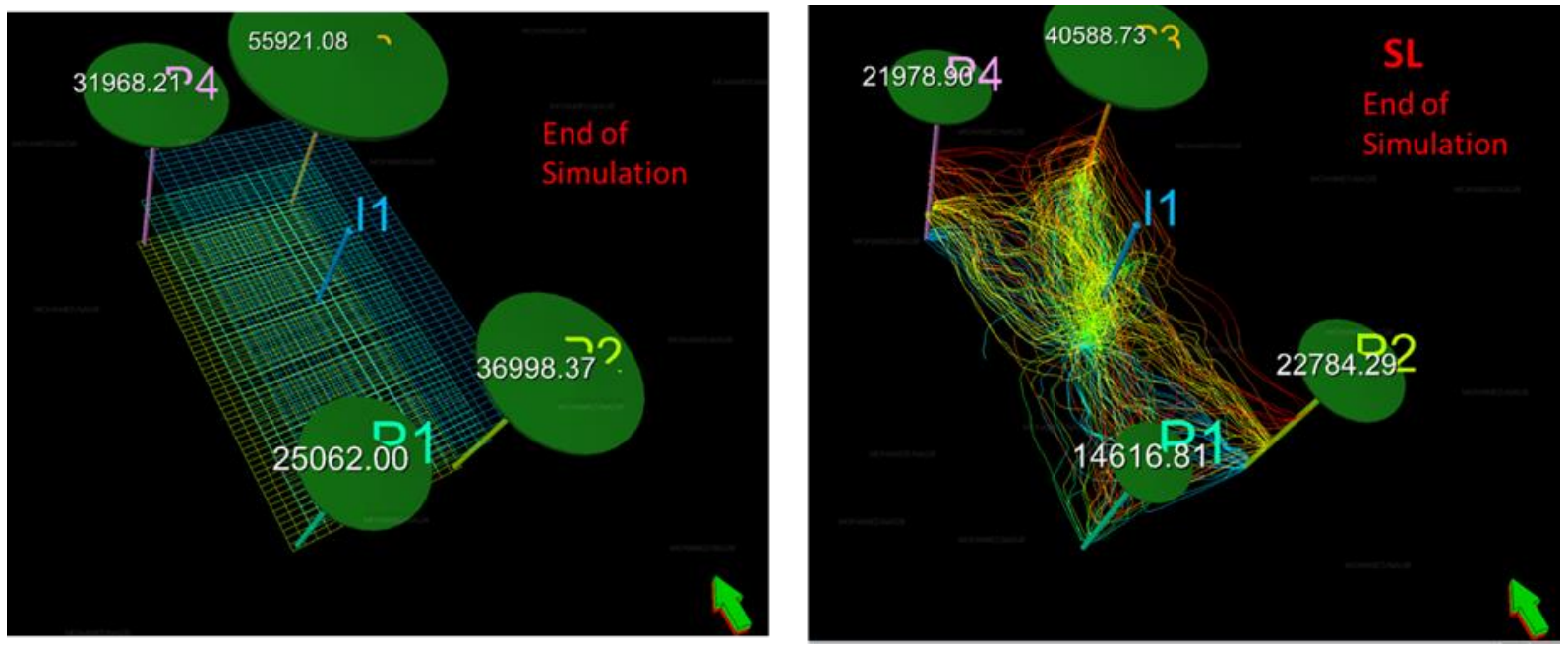

Figure 9-17: Total oil production per each well with 3D grid of the high-resolution model.

Similar to the base case model, production data per each well are comparable and very close. 
Figure 9-18 shows the compositional model. As per the graph below, to the left is shown the total oil production per each producer of the FD model, and to the right is shown the same for SL model, both at the end of simulation.
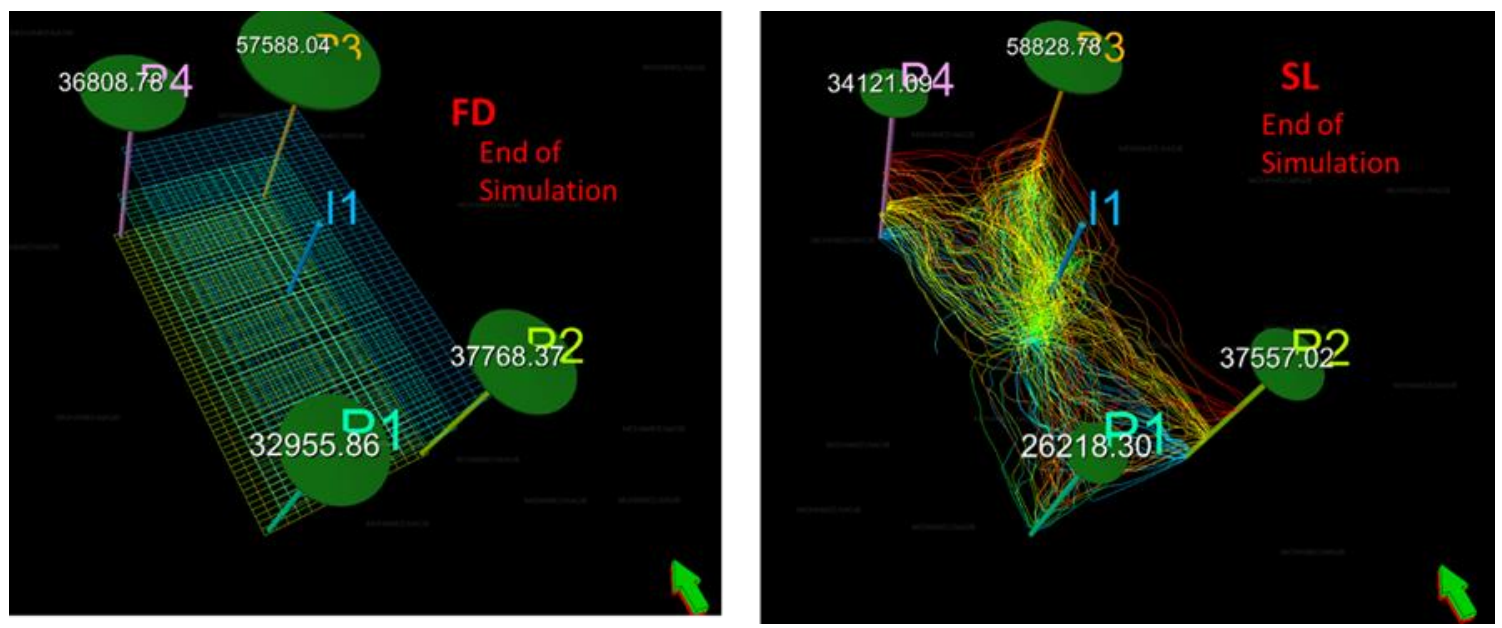

Figure 9-18: Total oil production per each well with 3D grid of the compositional model.

As noticed in the above graph, All producers in compositional SL model are producing high in comparison to base case and high-resolution cases in addition to the FD compositional model.

Figure 9-19 shows the Miscible flood model. As per the graph below, to the left is shown the total oil production per each producer of the FD model, and to the right is shown the same for the SL model, both at the end of simulation.
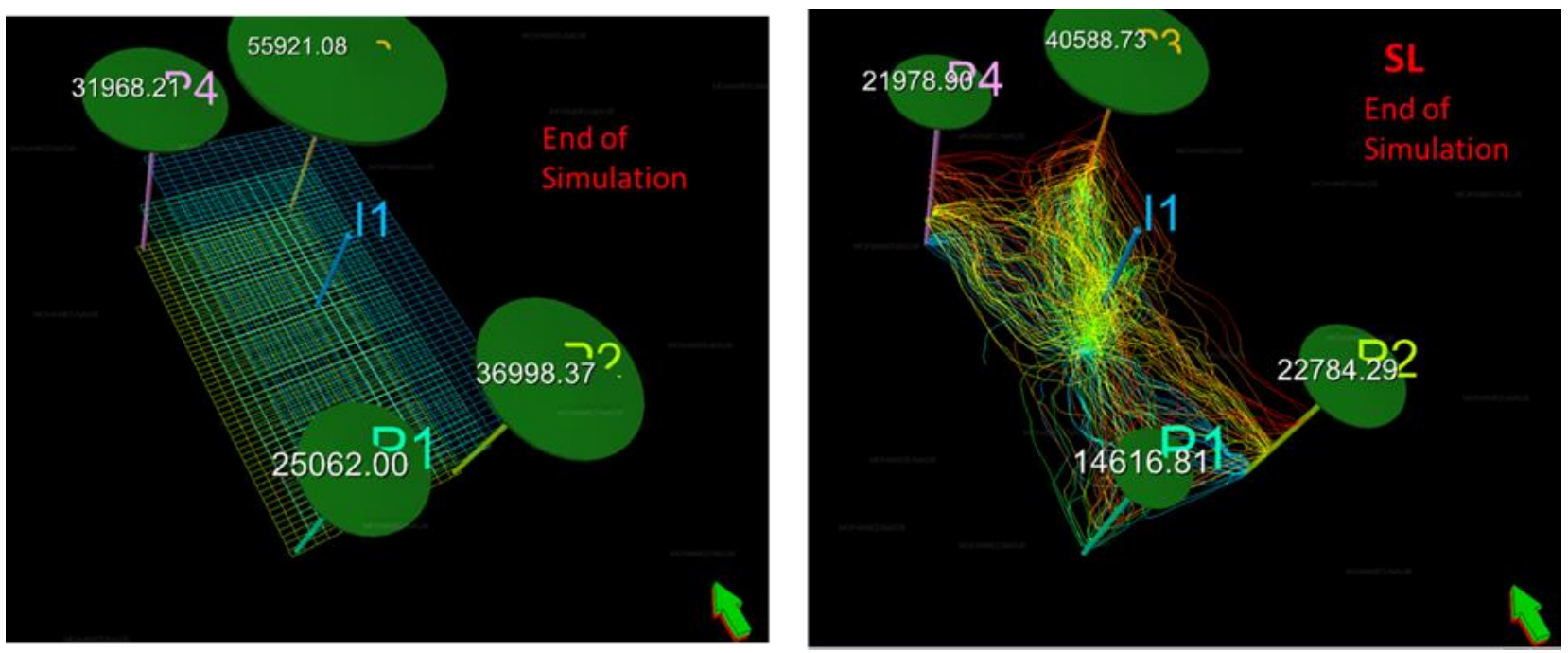
Figure 9-19: Total oil production per each well with 3D grid of the Miscible model.

\subsection{Conclusions}

- Streamline based simulator was extended to study and test the behaviour / performance of the streamline-based simulation for the gas displacement processes and compare it with the industry standard finite difference simulation both Black Oil and Compositional.

- The results indicate that SL simulation can be used for gas displacement processes. The cases investigated showed that a careful choice of simulation tuning parameters maintains the speed advantage of SL simulation, without wholly compromising the accuracy of the simulation results, compared with FD.

- The evaluation criteria included the down-hole conditions, well productivities and simulator performance indicators.

- In the depletion case (no gas injection), SL simulation generally works as a front tracking tool, and in this case there is no fluid injected into the reservoir. The results are not perfectly matched, and the SL simulation model produces generally less than FD simulation.

- Sensitivities on different gas injection rates showed the real impact of changing the gas injection rates, and both FD and SL simulation models represent the same effect of changing the injection rates.

- The bottom hole pressure sensitivity results showed that SL is controlled and affected more by the changing in bottom hole pressure, as once bottom hole pressure dropped to a certain level, the SL simulation lost control and the model stopped.

- A high-resolution grid of the same base case gas injection model was developed by creating a new grid with the properties and increasing the number of grid cells in the $\mathrm{x}$ and $\mathrm{y}$ directions by twice the number of the base case.

- The tuning parameters were kept the same in this case in order to see the quality and the degree of match between the base case and high resolution grid. 
- In order to improve SL performance model and hence reduce the relative error between SL and FD simulation models, well control models need to be refined again in both models to minimise the difference, especially in the initial production rates. If the initial production rates are similar, this will result in less relative error.

- Evaluation criteria was included intensive 3D grid analysis of both FD and SL cases studies as in the case of base case gas injection, High Resolution case, Compositional and Miscible flood cases.

- OTC calculated for all cases bases upon 3D oil saturation analysis and results showed that in most of the cases, SL models behaving similar to FD models with some minor differences.

- OTC differences between SL and FD also confirmed minor differences as shown in OTC 3D grids.

- Both Compositional and Miscible Gas injection showed the least error difference, and a fairly high accuracy using an optimised choice of simulation tuning parameters. In both cases, the same tuning parameters as for the baseline case were used as well. CPU time requirements and numerical dispersion were controlled and reduced significantly, while allowing the engineer to use a finely gridded model capturing the heterogeneity of the reservoir.

\subsection{Future work}

The streamline simulation method is conventionally applied to incompressible fluids, and in this study we extended the use of it for compressible fluids. Both compositional streamline simulation and miscible gas injection where $\mathrm{CO}_{2}$ is slightly compressible were tested in this work with some limitations. Therefore, it is necessary later on to include compressibility in order to accurately capture the right physics. 


\section{References}

1. Lake, L.W. and Walsh, M.B. Enhanced Oil Recovery (EOR) field data litrature search (2008).

2. Habermann, B. The efficiency of miscible displacement as a function of mobility ratio, Trans AIME 219 (1960).

3. Lacey, J.W., Faris. J.E. and Brinkman, F.H. Effect of bank size on oil recovery in the high pressure gas-driven LPG Bank Process, JPT, 13, AH (1961).

4. Perrine, R.L. The Development of Stability Theory for Miscible Liquid - Liquid Displacement, SPE J1, (1961).

5. Dollberry, G. Gary Dolberry on Enhanced Oil Recovery. [Online] 24 August 2011. [Cited: 24 August 2012] http://orangecast.hubpages.com/hub/Gary-Dolberry-on-Enhanced-Oil-Recovery (2008)

6. MDM Energy INC, Geology and History of Crawfrd County, MDM Energy.Com. [Online] MDM Energy INC., 24 August 2012. [Cited: 24 August 2012]. http://www.mdmenergy.com/crawford.html.

7. Petro Views, Enhanced Oil Recovery, [Online] [Cited: 24 August 2012]. http://petroviews.blogspot.com/2011/06/enhanced-oil-recovery-technology.html.

8. China Oil Field Technology, Chemical Flooding, [Online] 24 August 2012. [Cited: 24 August 2012] http://www.chinaoilfieldtech.com/chemicalflooding.html.

9. Datta-Gupta, A. and King, M.J Streamline Simulation, Theory and Practice, SPE Text Book series (2007).

10. NETL: E\&P. Exploration \& Production Technologies. NETL: E\&P. [Online] 24 August 2012. [Cited: $24 \quad$ August 2012] 24 ttp;//www.netl.doe.gov/technologies/oil-gas /EP_Technologies/ImprovedRecovery/EnhancedOilRecovery/GasFlood.html.

11. Emerson Process Management, Enhance oil Recovery, [Online] 24 August 2011. [Cited: 24 August 2011.] http://www.emersonprocess.com/en-US/brands/micromotion/industries/oil-andgas/upstream-production/enhanced-oil-recovery/Pages/index.aspx.

12. Pletcher, R.R., Tannehill, J.C. and Anderson, D.D. Computational Fluid Mechanics and Heat Transfer, CRC Press (1972). 
13. Neumann, J.V., Collected works, IV, Macmillan Co., New York, NY (1963).

14. O'Brien, B.G., Hyman M.A. and Kaplan, S. A study of the Numerical Solution of Partial Difference Equations, J. Math. Phy. 29, 223 (1951).

15. Hilderband, F.B., Finite difference Equations and Simulations, Prentice Hall (1968).

16. Mattax, C.C. and Dalton, R.L. Reservoir simulation, Society of Petroleum Engineers, Technology \& Engineering. Chapter 1, 2 and 5. Finite Difference for fractional Flow Equation (1990).

17. Higgins, R.V. and Leighton, A.J. Waterflood performance in stratified reservoirs: recovery as influenced by relative permeability curves and by continuously changing saturation (1960).

18. Lolomari, T., Bratvedt K., Crane, M., Milliken, W.J. and Tyrie J.J. The Use of Streamline simulation in Reservoir Management: Methodology and Case Studies. SPE 63157 in the proceeding of the SPE Annual Technical Conference and Exhibition, Dallas, Texas, U.S.A., 1-4 October 2000.

19. Crane, M., Bratvedt, F., Bratvedt, K. and Childs, P. A fully Compositional Streamline Simulator. SPE 63156 in the proceeding of the SPE Annual Technical Conference and Exhibition, Dallas, Texas, U.S.A., 1-4 October 2000.

20. Baek, M. and Hewett, T.A. A Hybrid Streamtube Simulator Using A Semianalytical Method, SPE Annual Technical Conference and Exhibition, 1-4 October 2000, Dallas, Texas SPE 63151-MS.

21. Al-Harbi, M., Cheng, H., He, Z. and Datta-Gupta, A. Streamline-based Production Data Integration in Naturally Fractured Reservoirs, SPE Journal (2005), 426-439.

22. Cheng, H., Oyerinde, D., Datta-Gupta, A. and Milliken, W. Compressible Streamlines and ThreePhase History Matching, paper SPE 99465 presented at the 2006.

23. Thiele, M.R., Batycky, R.P. and Fenwick, D.H. Streamline Simulation for Modern Reservoir Engineering Workflows. Texas: JPT/SPE, 2010, Vol. II. SPE 118608.

24. Batycky, R.P., Förster, M., Thiele, M.R., and Stüben. Parallelization of a Commercial Streamline Simulator and Performance on Practical Models. Texas, USA : SPE, 2009, SPE 118684.

25. Thiele, M.R. and Batycky, R.P. Streamsim Technologies, Water Injection Optimization Using a Streamline-Based Workflow, paper SPE 84080 presented at SPE Annual Technical Conference and Exhibition, 5-8 October 2003, Denver, Colorado.

26. Naguib, M. and Batycky, R. Results of Proactively Managing a Heavy-Oil Waterflood in South Oman Using Streamline-Based Simulation. SPE-101195 presented at SPE Asia Pacific Oil \& Gas Conference and Exhibition, 11-13 September 2006, Adelaide, Australia. 
27. Thiele, M.R., Batycky, R.P. and Blunt, M.J. A Streamline-Based 3D field-Scale Comporisitonal Reservoir Simulator. SPE Paper 38889 presented at SPE Annual Technical Conference and Exhibition, San Antonio, Texas 5-8 October 1997.

28. Crane, M., Bratvedt, F., Bratvedt, K. and Childs P. A fully Compositional Streamline Simulator. SPE 63156 in the proceeding of the SPE Annual Technical Conference and Exhibition, Dallas, Texas, U.S.A., 1-4 October 2000.

29. Jessen, K., and Orr Jr., F.M., Compositional streamline Simulation" SPE Paper 77379 presented at the SPE Annual Technical Conference and Exhibition San Antonio, Texas, $29^{\text {th }}$ September- $2^{\text {nd }}$ of October 2002.

30. Jessen, K., and Orr Jr., F.M., Gravity Segregation and Compositional Streamline Simulation SPE Paper 89448 presented at the SPE/DOE Symposuim on Improved Oil Recovery, Tulsa, Oklahoma, 17-29 April 2004.

31. Jemenez, E., Sabir, K. and Datta-Gupta, A. Spatial Error and Convergence in Streamline Simulation. SPE paper 92873 presented at the SPE Reservoir Simualtion Sympsuim, Woodlands, Texas, U.S.A 31 January - 02 February 2005.

32. Cheng, H., Oyerinde, D., Datta-Gupta, A. and Milliken, W. Compressible Streamlines and ThreePhase History Matching, SPE Paper 99465 presented at SPE/DOE Symposuim on Improved Oil Recovery, Tulsa, Oklahoma, 22-26 April 2006.

33. Beraldo, V.T., Blunt, M.J. and Schiozer, D.J. Compressible Streamline-Based Simulation with Changes in Oil Composition, Proceedings of the SPE Annual Technical Conference and Exhibition Denver, Colorado, 21-24 September, 2008.

34. Lazaro-Vallejo, L., Leahy, M., Dance, T. and LaForce, T. New phase Behavior Algorithm for Simulation of $\mathrm{CO}_{2}$ Storage, Paper SPE 90136 presented at the SPE Reservoir Simualtion Sympsuim, Woodlands, Texas, U.S.A, 21 - 23 February 2011.

35. Tanaka, S., Gupta, A.D. and King, M.J., Compositional Streamline Simulation of $\mathrm{CO}_{2}$ Injection Accounting for Gravity and Capillary Effects Using Orthogonal Projection, Paper SPE 169066 presented at the SPE Improved Oil Recovery Symposium held in Tulsa, Oklahoma, U.S.A, 12-14 April 2014.

36. Schlumberger, [Online] 22 August 2012. [Cited: 22 August 2012.] http://www.slb.com/ /media/Files/software/product_sheets/eclipse2010_wnu_ps.ash. 
37. Schlumberger, Eclipse / Frontsim Technical Description Manual.

38. 3DSL, User Manual, Streamsim Technologies.

39. Christie, M.A. and Blunt, M. J., Tenth SPE comparative solution Project: A comparison of upscaling technique. Houston, TX: SPE, (2001), Vol. 10. SPE 66599.

40. Batycky, R.P., Blunt, M.J. and Thiele, M.R. s.l. A 3D Field-Scale Streamline Based-Reservoir Simulator, SPERE12, 1997. SPE-36726-PA.

41. Batycky, R.P., Förster, M., Thiele, M.R. and Stüben, Parallelization of a Commercial Streamline Simulator and Performance on Practical Models, Texas, USA : SPE, 2009. SPE 118684.

42. Batycky, R.P. and Thiele, M.R. Water Injection Optimization Using a Streamline-Based Workflow, paper SPE 84080 presented at SPE Annual Technical Conference and Exhibition, 5-8 October 2003, Denver, Colorado.

43. Baker, R. Streamline Technology: Reservoir History Matching and Forecasting = Its Success, Limitations, and Future, Journal of Canadian Petroleum Technology, Volume 40, Number 4 (2001).

44. Pasarai, Arihara, N. SPE, Waseda U.A Simulator for Predicting Thermal Recovery Behavior Based on Streamline Method SPE-97411-MS Author U SPE 97411-MS 2005.

45. Todd. M.R., O'Dell, P.M. and Hirasaki, G.J., Methods for Increased Accuracy in Numerical Reservoir Simulators, Soc. Pet. Eng. J. (1972), 515-539.

46. Varga, R.S. Matrix Iterative Analysis, Prentice-Hall, Englewood Cliffs, NJ (1962).

47. Lake, L.W. Enhanced Oil Recovery, Prentice-Hall, Inc. (1989).

48. Dake, L.P. Fundamentals of Reservoir Engineering, Elsevier (1978).

49. Cheng, H., Osako, I., Datta-Gupta, A. and King, M. J. A Rigorous Compressible Streamline Formulation for Two- and Three-Phase Black-Oil Simulation, SPE Journal, 11(4), 407-417, (2006).

50. Christie, M.A. and Blunt, M.J., Tenth SPE Comparative Solution Project: A Comparison of Upscaling Techniques, SPE Reservoir Evaluation and Engineering, 4(4), 308-317, (2001).

51. Beraldo, V.T., Blunt, M.J. and Schiozer, D.J. Compressible Streamline-Based Simulation with Changes in Oil Composition, Proceedings of the SPE Annual Technical Conference and Exhibition Denver, Colorado, 21-24 September, 2008. 
52. Batycky, R.P. A Three-dimensional Two-phase Field Scale Streamline Simulatior, PhD thesis, Stanford University (1997).

53. Batycky, R.P., Blunt, M.J. and Thiele, M.R., A 3D Field-scale Streamline-based Reservoir Simulator, SPE Reservoir Engineering, 12(4), 246-254, 1997.

54. Pollock, D.W., Semi-Analytical Computation of Pathlines for Finite difference Models, Groundwater, 26(b), 743-750, 1998.

55. Obi, E., Streamline-Based Simulation of Contaminant Transport, PhD thesis, Imperial College London (2004).

56. Osako, I. and Datta-Gupta, A. A Compositional Streamline Formulation With Compressibility Effects, SPE 106148, Proceedings of the SPE Reservoir Simulation Symposium, Houston, Texas, 26-28 February, 2007.

57. Dickey, P. A. Petroleum Development Geology. $3^{\text {rd }}$ ed. Tulsa, OK, PennWell Books (1986).

58. Olewczyńska, M., Grötsch, J. and Al Jundi, J. High-Resolution Static/Dynamic Modelling and 3Phase Streamline Simulation in Complex Fluviatile Reservoirs, SPE 10896, proceedings of IPTC conference, 21-23 November 2005, Doha, Qatar.

59. Theile, M., Batycky, R.P, Poliilizer, S. and Clemens, T. Polymer-Flood Modelling Using Streamlines, SPE Reservoir Evaluation \& Engineering Volume 13, Number 2, (2010).

60. Haegland, H., Dahle, H. K., Eigestad, G.T. and Lie, K. Improved streamlines and time-of-flight for streamline simulation on irregular grids, Advances in Water Resources 30 (2007) 1027-1045.

61. Watanabe, S. and Data-Gupta, A. Use of phase Streamlines for Covariance Localization in Ensemble Kalaman Filter for Three-Phase History Matching, SPE Reservoir Evaluation \& Engineering, Volume 15, Number 3, 2012.

62. Clemens, T., Abdev, J. and Thiele, M.R. Improved Polymer-Flood Management Using Streamlines. SPE Reservoir Evaluation \& Engineering, Volume 14, Number 2, (2011) 171-181.

63. De Haan, H.J. Compania Shell de Venezuela Ltd., van Lookeren, J. Compania Shell de Venezuela Ltd. Early Results of the First Large-Scale Steam Soak Project in the Tia Juana Field, Western Venezuela, Journal of Petroleum Technology, Volume 21, Number 1, 1969 
64. Al-Najem, A.A., Siddiqui, S., Soliman, M. and Yuen, B. Streamline Simulation Technology: Evolution and Recent Trends, Paper SPE 160894 presented at the SPE Saudi Arabia Section Technical Symposium and Exhibition, Al-Khobar, Saudi Arabia, 8-11 April 2012.

65. AL-Zaway, A.S., Hayder, E.M., Baddourah, M.A., Mohd Ghazali, B.A. and Hidayat, W. Using Streamline and Reservoir Simulation to Improve Water Flood Management. Paper SPE 141794 presented at the SPE Middle East Oil and Gas Show Conference and Exhibition, Manama, Bahrain 25-28 September 2010.

66. Wang, H., Zhao, W., Espedal, M.S. and Teyakovskiv, A.S. A Component-Based Eulerian-Lagrangian Formulation for Multicomponent Multiphase Compositional Flow and Transport in Porous Media, SIAM Journal on Scientific Computing, Volume 35(2), B462-B486 (2013).

67. Wang J., Si, Z. and Sun, W. A New Error Analysis of Characteristics-Mixed FEMs for Miscible Displacement in Porous Media, SIAM Journal on Numerical Analysis 52:6, 3000-3020 (2014).

68. Qi, R. Simulation of Geological Carbon Dioxide Storage, PhD Thesis, Imperial College (2009).

69. Zhang, Y., Yang, C., King, M., and Datta-Gupta A., Fast Marching Methods for Complex Grids and Anisotropic Permeabilities: Application to Unconventional Reservoirs. Paper SPE 163637 presented at the SPE Reservoir Simulation Symposium held in woodlands, Texas USA, 18-20 February 2013.

70. Lino, A., Vyas, A., Huang, J. and Datta-Gupta A. Rapid compositions Simulation and History Matching of Shale Oil Reservoir Using the Fast Marching Method. Paper URTeC 2693139 presented at Unocnventional Resources Technology Conference, Austin, Texas, USA 24-26 July 2017.

71. Tanaka, S., Kam, D., Wang, J. and Datta-Gupta A. A Generalized Derivative-Free Rate Allocation Optimisation for Water and Gas Flooding Using Streamline-Based Method. Paper SPE 187298 at the SPE Annual Technical confernce and Exhibtion, San Antonio, Texas, USA 9-11 October 2017.

72. Lino, A., and Datta-Gupta, A., Optimising $\mathrm{CO}_{2}$ and field gas injection EOR in Unconventional Reservoirs using Fast Marching Method. Paper SPE 190304 at the SPE Improved Oil Recovery Confernce, Tulsa, Oklahoma, USA 14-18 April 2018.

73. Bhambri, P. and Mohanty, K. Compositional Streamline Simulation: A Parallel implementation, Paper 90:741-761 Transport of Porous Media Journal (2011).

74. Yan, W., Yan, W., Michelsen, M.L., Berenblyum, R.A. and Shapiro, A.A. Three phase compositional streamline simulation and its application to WAG, SPE 89440. Paper presented at SPE/DOE symposium on improved oil recovery, Tulsa (2004). 
75. Baker, R.O., Kuppe, F., Chugh, S., Bora, R., Stojanovic, S. and Batycky, R. (2001). Full-Field Modeling Using Streamline-Based Simulation: 4 Case Studies. Society of Petroleum Engineers, doi:10.2118/66405-MS.

76. Olewczynska, M.M., Grotsch, J., Al-Jundi, J. and Rao, S. High-Resolution Static/Dynamic Modelling and 3-Phase Streamline Simulation in Complex Fluviatile Reservoirs. International Petroleum Technology Conference. doi:10.2523/IPTC-10896-MS.

77. Butcher, J. C., The numerical analysis of ordinary differential equations, Runge-Kutta and general linear methods, Wiley, Chichester and New York (1987).

78. Coats, K. H. (2003). IMPES Stability: Selection of Stable Timesteps. Society of Petroleum Engineers. doi:10.2118/84924-PA.

79. Todd, M. and Longstaff, W. The Development, Testing and Application of a Numerical Simulator for Predicting Miscible Flood Performance, paper SPE 3484, Journal of Canadian Petroleum Technology (1972) 24, No. 7, 874-882. 
Appendix- A

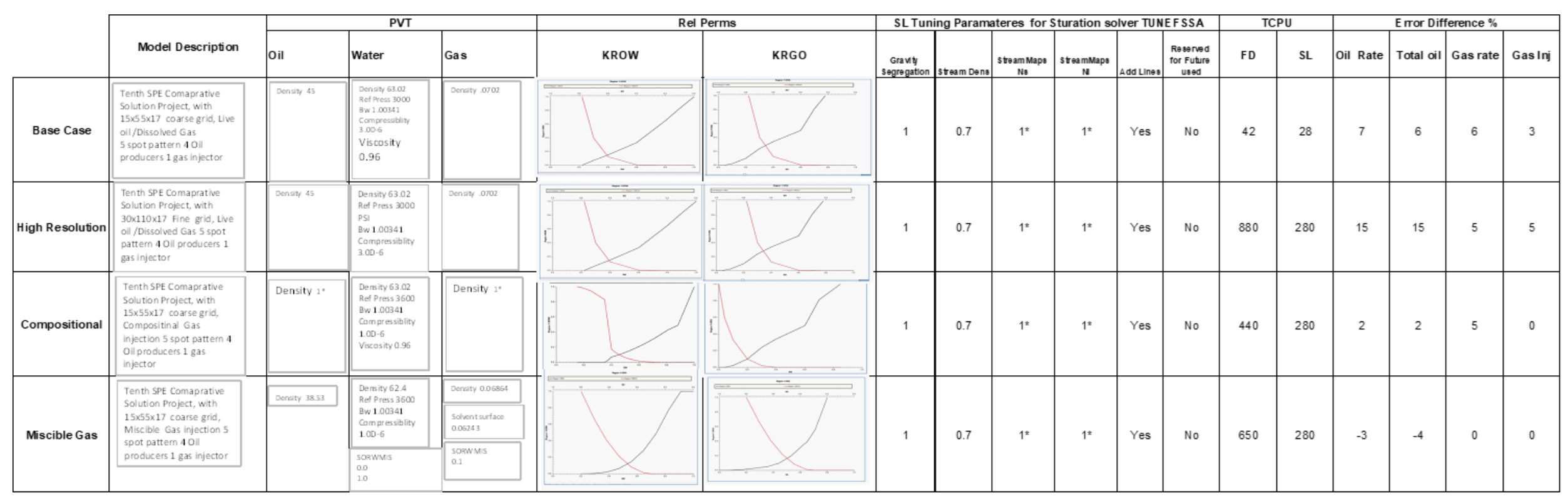

